PREFACE

PERCOLATION AND

DISORDERED SYSTEMS

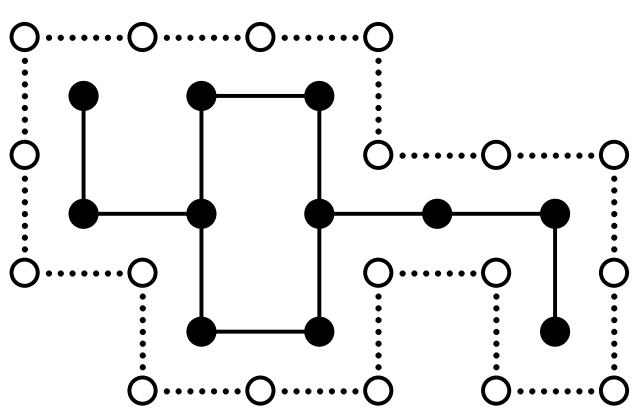

Geoffrey GRIMMETT
This course aims to be a (nearly) self-contained account of part of the mathematical theory of percolation and related topics. The first nine chapters summarise rigorous results in percolation theory, with special emphasis on results obtained since the results in percolation theory, with special emphasis on results obtained since the
publication of my book [156] entitled 'Percolation', and sometimes referred to simply as $[G]$ in these notes. Following this core material are chapters on random walks in random labyrinths, and fractal percolation. The final two chapters include material on Ising, Potts, and random-cluster models, and concentrate on a 'percolative' approach to the associated phase transitions.

The first target of this course is to draw a picture of the mathematics of percolation, together with its immediate mathematical relations. Another target is to colation, together the first nine chapters and the contents of the principal reference [G]. On the othe hand, the current notes are more concise than $[\mathrm{G}]$, and include some important extensions, such as material concerning strict inequalities for critical probabilities, the uniqueness of the infinite cluster, the triangle condition and lace expansion in high dimensions, together with material concerning percolation in slabs, and conformal invariance in two dimensions. The present account differs from that of $[\mathrm{G}] \mathrm{in} \mathrm{nu}-$ merous minor ways also. It does not claim to be comprehensive. A second edition of $[\mathrm{G}]$ is planned, containing further material based in part on the current notes.

A special feature is the bibliography, which is a fairly full list of papers published in recent years on percolation and related mathematical phenomena. The compilation of the list was greatly facilitated by the kind responses of many individuals to my request for lists of publications.

Many people have commented on versions of these notes, the bulk of which have been typed so superbly by Sarah Shea-Simonds. I thank all those who have contributed, and acknowledge particularly the suggestions of Ken Alexander, Carol Bezuidenhout, Philipp Hiemer, Anthony Quas, and Alan Stacey, some of whom are mentioned at appropriate points in the text. In addition, these notes have benefite from the critical observations of various members of the audience at St Flour.

Members of the 1996 summer school were treated to a guided tour of the library of the former seminary of St Flour. We were pleased to find there a copy of the Encyclopédie, ou Dictionnaire Raisonné des Sciences, des Arts et des Métiers, compiled by Diderot and D'Alembert, and published in Geneva around 1778. Of the many illuminating entries in this substantial work, the following definition of a probabilist was not overlooked. 
PROBABILISTE, s. m. (Gram. Théol.) celui qui tient pour la doctrine abominable des opinions rendues probables par la décision d'un casuiste, \& qui assure l'innocence de l'action faite en conséquence. Pascal a foudroyé ce systême, qui ouvroit la porte au crime en accordant à l'autorité les prérogatives de la certitude, à l'opinion \& la sécurité qui n'appartient qu'à la bonne conscience.

This work was aided by partial financial support from the European Union under contract CHRX-CT93-0411, and from the Engineering and Physical Sciences Research Council under grant GR/L15425.

Geoffrey R. Grimmett

Statistical Laboratory

University of Cambridge

16 Mill Lane

Cambridge CB2 $1 S B$

United Kingdom.

October 1996

1. Introductory Remarks

1.1 Percolation

1.2 Some possible questions

1.3 History

2. Notation and Definitions

2.1 Graph terminology

2.2 Probability

A partial orde

2.4 Site percolation

3. Phase Transition

3.1 Percolation probability

3.2 Existence of phase transition

3.3 A question

Inequalities for Critical Probabilities

4.1 Russo's formula

4.2 Strict inequalities for critical probabilities

4.3 The square and triangular lattices

4.4 Enhancements

5. Correlation Inequalities

5.1 FKG inequality

5.2 Disjoint occurrence

5.3 Site and bond percolation

6. Subcritical Percolation

6.1 Using subadditivity

6.2 Exponential decay

6.3 Ornstein-Zernike decay

\section{Supercritical Percolation}

7.1 Uniqueness of the infinite cluster

7.2 Percolation in slabs

7.3 The block construction

7.4 Limit of slab critical points

7.5 Percolation probability

7.6 Cluster-size distribution

CONTENTS 
8. Critical Percolation

8.1 Percolation probability

8.2 Critical exponent

8.3 Scaling theory

8.4 Rigorous results

8.5 Mean-field theory

9. Percolation in Two Dimensions

9.1 The critical probability is $\frac{1}{2}$

9.2 RSW technology

9.3 Conformal invariance

0. Random Walks in Random Labyrinth

10.1 Random walk on the infinite percolation cluste

10.2 Random walks in two-dimensional labyrinths

10.3 General labyrinth

\section{Fractal Percolation}

1.1 Random fractals

11.2 Percolation

11.3 A morphology

1.4 Relationship to Brownian Motion

\section{Ising and Potts Models}

12.1 Ising model for ferromagnets

12.2 Potts models

12.3 Random-cluster models

\section{Random-Cluster Models}

13.1 Basic properties

13.2 Weak limits and phase transitions

13.3 First and second order transitions

13.4 Exponential decay in the subcritical phase

13.5 The case of two dimensions

\section{INTRODUCTORY REMARKS}

1.1. Percolation

We will focus our ideas on a specific percolation process, namely 'bond percolation on the cubic lattice', defined as follows. Let $\mathbb{L}^{d}=\left(\mathbb{Z}^{d}, \mathbb{E}^{d}\right)$ be the hypercubic lattice in $d$ dimensions, where $d \geq 2$. Each edge of $\mathbb{L}^{d}$ is declared open with probability $p$ and closed otherwise. Different edges are given independent designations. We think of an open edge as being open to the transmission of disease, or to the passage of water. Now concentrate on the set of open edges, a random set. Percolation theory is concerned with ascertaining properties of this set.

The following question is considered central. If water is supplied at the origin, and flows along the open edges only, can it reach infinitely many vertices with strictly positive probability? It turns out that the answer is no for small $p$, and yes for large $p$. There is a critical probability $p_{\mathrm{c}}$ dividing these two phases. Percolation theory is particularly concerned with understanding the geometry of open edges in the subcritical phase (when $p<p_{\mathrm{c}}$ ), the supercritical phase (when $p>p_{\mathrm{c}}$ ), and when $p$ is near or equal to $p_{\mathrm{c}}$ (the critical case).

As an illustration of the concrete problems of percolation, consider the function $\theta(p)$, defined as the probability that the origin lies in an infinite cluster of open edges (this is the probabity referrod to above, in the discussion of $p_{c}$ ). It is believed that

- $\theta$ should be smooth on $\left(p_{\mathrm{c}}, 1\right)$. It is known to be infinitely differentiable, but there is no proof known that it is real analytic for all $d$.

- Presumably $\theta$ is continuous at $p_{\mathrm{c}}$. No proof is known which is valid for all $d$.

- Perhaps $\theta$ is concave on $\left(p_{\mathrm{c}}, 1\right]$, or at least on $\left(p_{\mathrm{c}}, p_{\mathrm{c}}+\delta\right)$ for some positive $\delta$.

- As $p \downarrow p_{\mathrm{c}}$, perhaps $\theta(p) \sim a\left(p-p_{\mathrm{c}}\right)^{\beta}$ for some constant $a$ and some "critical exponent' $\beta$.

We stress that, although each of the points raised above is unproved in general, there are special arguments which answer some of them when either $d=2$ or $d$ is sufficiently large. The case $d=3$ is a good one on which to concentrate.

1.2 Some Possible Questions

Here are some apparently reasonable questions, some of which turn out to be feasible

- What is the value of $p_{\mathrm{c}}$ ?

- What are the structures of the subcritical and supercritical phases?

- What happens when $p$ is near to $p_{\mathrm{c}}$ ?

- Are there other points of phase tra

- What are the properties of other 'macroscopic' quantities, such as the mean size of the open cluster containing the origin?

- What is the relevance of the choice of dimension or lattice?

- In what ways are the large-scale properties different if the states of nearby edges are allowed to be dependent rather than independent?

There is a variety of reasons for the explosion of interest in the percolation model, and we mention next a few of these. 


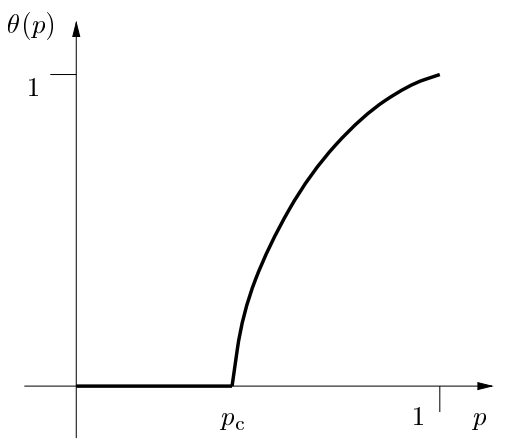

Fig. 1.1. It is generally believed that the percolation probability $\theta(p)$ behave tiable except at the critical point $p_{c}$. The possibility of a jump discontinuity at $p_{c}$ has not been ruled out when $d>3$ but $d$ is not too large.

- The problems are simple and elegant to state, and apparently hard to solve.

- Their solutions require a mixture of new ideas, from analysis, geometry, and discrete mathematics.

- Physical intuition has provided a bunch of beautiful conjectures.

- Techniques developed for percolation have applications to other more complicated spatial random processes, such as epidemic models.

- Percolation gives insight and method for understanding other physical models of spatial interaction, such as Ising and Potts models.

- Percolation provides a 'simple' model for porous bodies and other 'transport' problems.

The rate of publication of papers on percolation and its ramifications is very high in the physics journals, although substantial mathematical contributions are rare The depth of the 'culture chasm' is such that few (if anyone) can honestly boast to understand all the major mathematical and physical ideas which have contributed to the subject.

\subsection{History}

In 1957, Simon Broadbent and John Hammersley [81] presented a model for a disordered porous medium which they called the percolation model. Their motivation was perhaps to understand flow through a discrete disordered system, such as particles flowing through the filter of a gas mask, or fluid seeping through the interstices of a porous stone. They proved in $[81,174,175]$ that the percolation model has a phase transition, and they developed some technology for studying the two phases of the process.

These early papers were followed swiftly by a small number of high quality articles by others, particularly [138, 181,339], but interest flagged for a period beginning around 1964. Despite certain appearances to the contrary, some individuals realised that a certain famous conjecture remained unproven, namely that the critical probability of bond percolation on the square lattice equals $\frac{1}{2}$. Fundamental rigorous progress towards this conjecture was made around 1976 by Russo [326] and Seymour and Welsh [338], and the conjecture was finally resolved in a famous paper of Kesten [200]. This was achieved by a development of a sophisticated mechanism for study[200]. This was achieved by a development of a sophisticated mechanism for study-
ing percolation in two dimensions, relying in part on path-intersection properties ing percolation in two dimensions, relying in part on path-intersection properties
which are not valid in higher dimensions. This mechanism was laid out more fully by Kesten in his monograph [202].

Percolation became a subject of vigorous research by mathematicians and physicists, each group working in its own vernacular. The decade beginning in 1980 saw the rigorous resolution of many substantial difficulties, and the formulation of concrete hypotheses concerning the nature of phase transition.

The principal progress was on three fronts. Initially mathematicians concentrated on the 'subcritical phase', when the density $p$ of open edges satisfies $p<p_{\mathrm{c}}$ (here and later, $p_{\mathrm{c}}$ denotes the critical probability). It was in this context that the correct generalisation of Kesten's theorem was discovered, valid for all dimension (i.e., two or more). This was achieved independently by Aizenman and Barsky [13] and Menshikov [268, 269].

The second front concerned the 'supercritical phase', when $p>p_{\mathrm{c}}$. The key question here was resolved by Grimmett and Marstrand [165] following work of Barsky, Grimmett, and Newman [50].

The critical case, when $p$ is near or equal to the critical probability $p_{c}$, remains largely unresolved by mathematicians (except when $d$ is sufficiently large). Progress has certainly been made, but we seem far from understanding the beautiful picture of the phase transition, involving scaling theory and renormalisation, which is displayed before us by physicists. This multifaceted physical image is widely accepted as an accurate picture of events when $p$ is near to $p_{\mathrm{c}}$, but its mathematical verification is an open challenge of the first order. 


\section{NOTATION AND DEFINITIONS}

2.1 Graph Terminology

We shall follow the notation of $[\mathrm{G}]$ whenever possible (we refer to [156] as [G]). The number of dimensions is $d$, and we assume throughout that $d \geq 2$. We write $\mathbb{Z}=\{\ldots,-1,0,1, \ldots\}$ for the integers, and $\mathbb{Z}^{d}$ for the set of all vectors $x=\left(x_{1}, x_{2}, \ldots, x_{d}\right)$ of integers. For $x \in \mathbb{Z}^{d}$, we generally denote by $x_{i}$ the $i$ th coordinate of $x$. We use two norms on $\mathbb{Z}^{d}$, namely

$$
|x|=\sum_{i=1}^{d}\left|x_{i}\right|, \quad\|x\|=\max \left\{\left|x_{i}\right|: 1 \leq i \leq d\right\}
$$

and note that

$$
\|x\| \leq|x| \leq d\|x\| .
$$

We write

$$
\delta(x, y)=|y-x| .
$$

Next we turn $\mathbb{Z}^{d}$ into a graph, called the $d$-dimensional cubic lattice, by adding edges $\langle x, y\rangle$ between all pairs $x, y \in \mathbb{Z}^{d}$ with $\delta(x, y)=1$. This lattice is denoted $\mathbb{L}^{d}=\left(\mathbb{Z}^{d}, \mathbb{E}^{d}\right)$. We use the usual language of graph theory. Vertices $x, y$ with $\delta(x, y)=1$ are called adjacent, and an edge $e$ is incident to a vertex $x$ if $x$ is an endpoint of $e$. We write $x \sim y$ if $x$ and $y$ are adjacent, and we write $\langle x, y\rangle$ for the corresponding edge. The origin of $\mathbb{L}^{d}$ is written as the zero vector 0 , and $e_{1}$ denotes the unit vector $e_{1}=(1,0,0, \ldots, 0)$

A path of $\mathbb{L}^{d}$ is an alternating sequence $x_{0}, e_{0}, x_{1}, e_{1}, \ldots$ of distinct vertices $x_{i}$ and edges $e_{i}=\left\langle x_{i}, x_{i+1}\right\rangle$. If the path terminates at some vertex $x_{n}$, it is said to connect $x_{0}$ to $x_{n}$, and to have length $n$. If the path is infinite, it is said to connect $x_{0}$ to $\infty$. A circuit of $\mathbb{L}^{d}$ is an alternating sequence $x_{0}, e_{0}, x_{1}, e_{1}, \ldots, e_{n-1}, x_{n}, e_{n}, x_{0}$ such that $x_{0}, e_{0}, \ldots, e_{n-1}, x_{n}$ is a path and $e_{n}=\left\langle x_{n}, x_{0}\right\rangle$; such a circuit has length $n+1$. Two subgraphs of $\mathbb{L}^{d}$ are called edge-disjoint if they have no edges in common, and disjoint if they have no vertices in common.

A box is a subset of $\mathbb{Z}^{d}$ of the form

$$
B(a, b)=\prod_{i=1}^{d}\left[a_{i}, b_{i}\right] \quad \text { for } a, b \in \mathbb{Z}^{d}
$$

where $\left[a_{i}, b_{i}\right]$ is interpreted as $\left[a_{i}, b_{i}\right] \cap \mathbb{Z}$ and it is assumed that $a_{i} \leq b_{i}$ for all $i$ Such a box $B(a, b)$ may be turned into a graph by the addition of all relevant edges from $\mathbb{L}^{d}$. A useful expanding sequence of boxes is given by

$$
B(n)=[-n, n]^{d}=\left\{x \in \mathbb{Z}^{d}:\|x\| \leq n\right\} .
$$

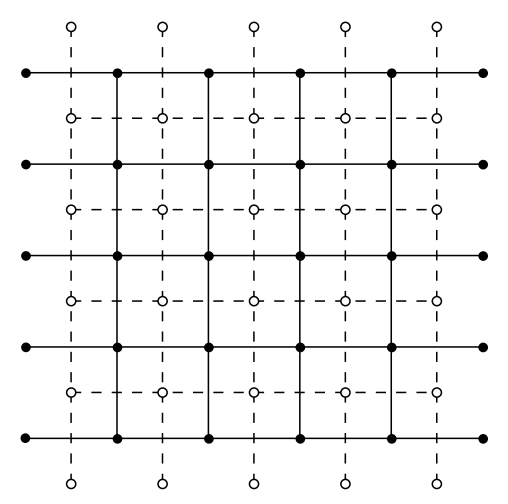

Fig. 2.1. Part of the square lattice $\mathbb{L}^{2}$ and its dual.

The case of two-dimensional percolation turns out to have a special property namely that of duality. Planar duality arises as follows. Let $G$ be a planar graph, drawn in the plane. The planar dual of $G$ is the graph constructed in the following way. We place a vertex in every face of $G$ (including the infinite face if it exists) and we join two such vertices by an edge if and only if the corresponding faces of and we join two such vertices by an edge if and only if the corresponding faces of
$G$ share a boundary edge. It is easy to see that the dual of the square lattice $\mathbb{L}^{2}$ $G$ share a boundary edge. It is easy to see that the dual of the square lattice $\mathbb{L}^{2}$
is a copy of $\mathbb{L}^{2}$, and we refer therefore to the square lattice as being self-dual. See Figure 2.1.

2.2 Probability

Let $p$ and $q$ satisfy $0 \leq p=1-q<1$. We declare each edge of $\mathbb{L}^{d}$ to be open with probability $p$, and closed otherwise, different ed ges having independent designations. The appropriate sample space is the set $\Omega=\{0,1\}^{\mathbb{E}^{d}}$, points of which are represented as $\omega=\left(\omega(e): e \in \mathbb{E}^{d}\right)$ called configurations. The value $\omega(e)=1$ corresponds to $e$ being open, and $\omega(e)=0$ to $e$ being closed. Our $\sigma$-field $\mathcal{F}$ is that generated by the finite-dimensional cylinders of $\Omega$, and the probability measure is product measure $P_{p}$ having density $p$. In summary, our probability space is $\left(\Omega, \mathcal{F}, P_{p}\right)$, and we write $E_{p}$ for the expectation operator corresponding to $P_{p}$.

\subsection{GEOMEtRY}

Percolation theory is concerned with the study of the geometry of the set of open edges, and particularly with the question of whether or not there is an infinite cluster of open edges.

Let $\omega \in \Omega$ be a configuration. Consider the graph having $\mathbb{Z}^{d}$ as vertex set, and as edge set the set of open edges. The connected components of this graph are called open clusters. We write $C(x)$ for the open cluster containing the vertex $x$, and call $C(x)$ the open cluster at $x$. Using the translation-invariance of $P_{p}$, we see that the distribution of $C(x)$ is the same as that of the open cluster $C=C(0)$ at the origin. 


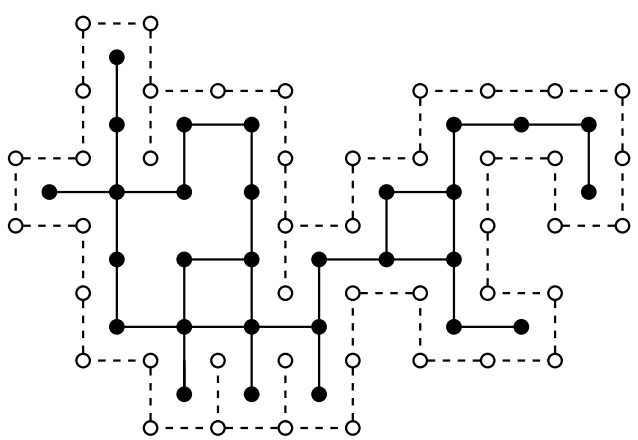

Fig. 2.2. An open cluster, surrounded by a closed circuit in the dual.

We shall be interested in the size of a cluster $C(x)$, and write $|C(x)|$ for the number of vertices in $C(x)$.

If $A$ and $B$ are sets of vertices, we write ' $A \leftrightarrow B$ ' if there is an open path (i.e., a path all of whose edges are open) joining some member of $A$ to some member of $B$. The negation of such a statement is written ' $A \leftrightarrow B$ '. We write ' $A \leftrightarrow \infty$ ' to mean that some vertex in $A$ lies in an infinite open path. Also, for a set $D$ of vertices (resp. edges), ' $A \leftrightarrow B$ off $D$ ' means that there is an open path joining $A$ to $B$ using no vertex (resp. edge) in $D$.

We return briefly to the discussion of graphical duality at the end of Section 2.1 . Recall that $\mathbb{L}^{2}$ is self-dual. For the sake of definiteness, we take as vertices of this dual lattice the set $\left\{x+\left(\frac{1}{2}, \frac{1}{2}\right): x \in \mathbb{Z}^{2}\right\}$ and we join two such neighbouring vertices by a straight line segment of $\mathbb{R}^{2}$. There is a one-one correspondence between the edges of $\mathbb{L}^{2}$ and the edges of the dual, since each edge of $\mathbb{L}^{2}$ is crossed by a unique edge of the dual. We declare an edge of the dual to be open or closed depending respectively on whether it crosses an open or closed edge of $\mathbb{L}^{2}$. This assignment gives rise to a bond percolation process on the dual lattice with the same edgeprobability $p$.

Suppose now that the open cluster at the origin of $\mathbb{L}^{2}$ is finite, and see Figure 2.2 for a sketch of the situation. We see that the origin is surrounded by a necklace of closed edges which are blocking off all possible routes from the origin to infinity. We may satisfy ourselves that the corresponding edges of the dual contain a closed circuit in the dual which contains the origin of $\mathbb{L}^{2}$ in its interior. This is best seen by inspecting Figure 2.2 again. It is somewhat tedious to formulate and prove such a statement with complete rigour, and we shall not do so here; see $[202, \mathrm{p} .386]$ for a more careful treatment. The convere holds similarly: if the origin is in the interior of a closed circuit of the dual lattice, then the open cluster at the origin is finite We summarise these remarks by saying that $|C|<\infty$ if and only if the origin of $\mathbb{L}^{2}$ is in the interior of a closed circuit of the dual.
2.3 A Partial Order

There is a natural partial order on $\Omega$, namely $\omega_{1} \leq \omega_{2}$ if and only if $\omega_{1}(e) \leq \omega_{2}(e)$ for all $e$. This partial order allows us to discuss orderings of probability measures on $(\Omega, \mathcal{F})$. We call a random variable $X$ on $(\Omega, \mathcal{F})$ increasing if

$$
X\left(\omega_{1}\right) \leq X\left(\omega_{2}\right) \quad \text { whenever } \omega_{1} \leq \omega_{2},
$$

and decreasing if $-X$ is increasing. We call an event $A$ (i.e., a set in $\mathcal{F}$ ) increasing (resp. decreasing) if its indicator function $1_{A}$, given by

$$
1_{A}(\omega)= \begin{cases}1 & \text { if } \omega \in A \\ 0 & \text { if } \omega \notin A\end{cases}
$$

is increasing (resp. decreasing)

Given two probability measures $\mu_{1}$ and $\mu_{2}$ on $(\Omega, \mathcal{F})$ we say that $\mu_{1}$ dominates $\mu_{2}$, written $\mu_{1} \geq \mu_{2}$, if $\mu_{1}(A) \geq \mu_{2}(A)$ for all increasing events $A$. Using this partial order on measures, it may easily be seen that the probability measure $P_{p}$ is non-decreasing in $p$, which is to say that

$$
P_{p_{1}} \geq P_{p_{2}} \quad \text { if } \quad p_{1} \geq p_{2} .
$$

General sufficient conditions for such an inequality have been provided by Holley [192] and others (see Holley's inequality, Theorem 5.5), but there is a simple direc proof in the case of product measures. It makes use of the following elementary device.

Let $\left(X(e): e \in \mathbb{E}^{d}\right)$ be a family of independent random variables each being uniformly distributed on the interval $[0,1]$, and write $P_{p}$ for the associated (product) measure on $[0,1]^{\mathbb{E}^{d}}$. For $0 \leq p \leq 1$, define the random variable $\eta_{p}=\left(\eta_{p}(e): e \in \mathbb{E}^{d}\right)$

$$
\eta_{p}(e)= \begin{cases}1 & \text { if } X(e)<p \\ 0 & \text { if } X(e) \geq p .\end{cases}
$$

It is clear that:

a) the vector $\eta_{p}$ has distribution given by $P_{p}$,

(b) if $p_{1} \geq p_{2}$ then $\eta_{p_{1}} \geq \eta_{p_{2}}$.

Let $A$ be an increasing event, and $p_{1} \geq p_{2}$. Then

$$
\begin{aligned}
P_{p_{1}}(A) & =P\left(\eta_{p_{1}} \in A\right) \geq P\left(\eta_{p_{2}} \in A\right) \quad \text { since } \eta_{p_{1}} \geq \eta_{p_{2}} \\
& =P_{p_{2}}(A),
\end{aligned}
$$

whence $P_{p_{1}} \geq P_{p_{2}}$ 
In bond percolation, it is the edges which are designated open or closed; in site percolation, it is the vertices. In a sense, site percolation is more general than bond percolation, since a bond model on a lattice $\mathcal{L}$ may be transformed into a site model on its 'line' (or 'covering') lattice $\mathcal{L}^{\prime}$ (obtained from $\mathcal{L}$ by placing a vertex in the middle of each edge, and calling two such vertices adjacent whenever the corresponding edges of $\mathcal{L}$ share an endvertex). See [138]. In practice, it matter little whether we choose to work with site or bond percolation, since sufficiently many methods work equally well for both models.

In a more general 'hypergraph' model, we are provided with a hypergraph on the vertex set $\mathbb{Z}^{d}$, and we declare each hyperedge to be open with probability $p$. We then study the existence of infinite paths in the ensuing open hypergraph.

We shall see that a percolation model necessarily has a 'critical probability' $p_{c}$. Included in Section 5.3 is some information about the relationship between the critical probabilities of site and bond models on a general graph $G$
3.1 Percolation Probability

One of the principal objects of study is the percolation probability

$$
\theta(p)=P_{p}(0 \leftrightarrow \infty)
$$

or alternatively $\theta(p)=P_{p}(|C|=\infty)$ where $C=C(0)$ is, as usual, the open cluster at the origin. The event $\{0 \leftrightarrow \infty\}$ is increasing, and therefore $\theta$ is non-decreasing (using (2.4)), and it is natural to define the critical probability $p_{\mathrm{c}}=p_{\mathrm{c}}\left(\mathbb{L}^{d}\right)$ by

$$
p_{\mathrm{c}}=\sup \{p: \theta(p)=0\} .
$$

See Figure 1.1 for a sketch of the function $\theta$.

\subsection{Existence of Phase Transition}

It is easy to show that $p_{\mathrm{c}}(\mathbb{L})=1$, and therefore the case $d=1$ is of limited interest from this point of view.

Theorem 3.2. If $d \geq 2$ then $0<p_{c}\left(\mathbb{L}^{d}\right)<1$

Actually we shall prove that

$$
\frac{1}{\mu(d)} \leq p_{\mathrm{c}}\left(\mathbb{L}^{d}\right) \leq 1-\frac{1}{\mu(2)} \quad \text { for } d \geq 2
$$

where $\mu(d)$ is the connective constant of $\mathbb{L}^{d}$.

Proof. Since $\mathbb{L}^{d}$ may be embedded in $\mathbb{L}^{d+1}$, it is 'obvious' that $p_{\mathrm{c}}\left(\mathbb{L}^{d}\right)$ is nonincreasing in $d$ (actually it is strictly decreasing). Therefore we need only to show that

$$
\begin{array}{ll}
(3.4) & p_{\mathrm{c}}\left(\mathbb{L}^{d}\right)>0 \quad \text { for all } d \geq 2, \\
(3.5) & p_{\mathrm{c}}\left(\mathbb{L}^{2}\right)<1 .
\end{array}
$$

The proof of (3.4) is by a standard 'path counting' argument. Let $N(n)$ be the number of open paths of length $n$ starting at the origin. The number of such path cannot exceed a theoretical upper bound of $2 d(2 d-1)^{n-1}$. Therefore

$$
\begin{aligned}
\theta(p) & \leq P_{p}(N(n) \geq 1) \leq E_{p}(N(n)) \\
& \leq 2 d(2 d-1)^{n-1} p^{n}
\end{aligned}
$$

which tends to 0 as $n \rightarrow \infty$ if $p<(2 d-1)^{-1}$. Hence $p_{\mathrm{c}}\left(\mathbb{L}^{d}\right) \geq(2 d-1)^{-1}$. By estimating $N(n)$ more carefully, this lower bound may be improved to

$$
p_{\mathrm{c}}\left(\mathbb{L}^{d}\right) \geq \mu(d)^{-1} .
$$


We use a 'Peierls argument' to obtain (3.5). Let $M(n)$ be the number of closed circuits of the dual, having length $n$ and containing 0 in their interior. Note that $|C|<\infty$ if and only if $M(n) \geq 1$ for some $n$. Therefore

$$
\begin{aligned}
1-\theta(p) & =P_{p}(|C|<\infty)=P_{p}\left(\sum_{n} M(n) \geq 1\right) \\
& \leq E_{p}\left(\sum_{n} M(n)\right)=\sum_{n=4}^{\infty} E_{p}(M(n)) \\
& \leq \sum_{n=4}^{\infty}\left(n 4^{n}\right)(1-p)^{n},
\end{aligned}
$$

where we have used the facts that the shortest dual circuit containing 0 has length 4 , and that the total number of dual circuits, having length $n$ and surrounding the origin, is no greater than $n 4^{n}$. The final sum may be made strictly smaller than 1 by choosing $p$ sufficiently close to 1 , say $p>1-\epsilon$ where $\epsilon>0$. This implies that $p_{\mathrm{c}}\left(\mathbb{L}^{2}\right)<1-\epsilon$.

This upper bound may be improved to obtain $p_{\mathrm{c}}\left(\mathbb{L}^{2}\right) \leq 1-\mu(2)^{-1}$. Here is a sketch. Let $F_{m}$ be the event that there exists a closed dual circuit containing the box $B(m)$ in its interior, and let $G_{m}$ be the event that all edges of $B(m)$ are open These two events are independent, since they are defined in terms of disjoint sets of edges. Now,

$$
P_{p}\left(F_{m}\right) \leq P_{p}\left(\sum_{n=4 m}^{\infty} M(n) \geq 1\right) \leq \sum_{n=4 m}^{\infty} n a_{n}(1-p)^{n}
$$

where $a_{n}$ is the number of paths of $\mathbb{L}^{2}$ starting at the origin and having length $n$ It is the case that $n^{-1} \log a_{n} \rightarrow \log \mu(2)$ as $n \rightarrow \infty$. If $1-p<\mu(2)^{-1}$, we may find It is the case that $n^{-1} \log a_{n} \rightarrow \log \mu$ such that $P_{p}\left(F_{m}\right)<\frac{1}{2}$. However,

$$
\theta(p) \geq P_{p}\left(\overline{F_{m}} \cap G_{m}\right)=P_{p}\left(\overline{F_{m}}\right) P_{p}\left(G_{m}\right) \geq \frac{1}{2} P_{p}\left(G_{m}\right)>0
$$

if $1-p<\mu(2)^{-1}$.

Issues related to this theorem include:

- The counting of self-avoiding walks (SAWS)

- The behaviour of $p_{\mathrm{c}}\left(\mathbb{L}^{d}\right)$ as a function of $d$.

- In particular, the behaviour of $p_{\mathrm{c}}\left(\mathbb{L}^{d}\right)$ for large $d$.

Kesten [200] proved that $p_{\mathrm{c}}\left(\mathbb{L}^{2}\right)=\frac{1}{2}$. This very special calculation makes essential use of the self-duality of $\mathbb{L}^{2}$ (see Chapter 9 ). There are various ways of proving the strict inequality

$$
p_{\mathrm{c}}\left(\mathbb{L}^{d}\right)-p_{\mathrm{c}}\left(\mathbb{L}^{d+1}\right)>0 \quad \text { for } d \geq 2,
$$

and good recent references include $[20,158]$.

On the third point above, we point out that

$$
p_{\mathrm{c}}\left(\mathbb{L}^{d}\right)=\frac{1}{2 d}+\frac{1}{(2 d)^{2}}+\frac{7}{2} \frac{1}{(2 d)^{3}}+\mathrm{O}\left(\frac{1}{(2 d)^{4}}\right) \quad \text { as } d \rightarrow \infty .
$$

See $[178,179]$, and earlier work of $[151,209]$

We note finally the canonical arguments used to establish that $0<p_{c}\left(\mathbb{L}^{d}\right)<1$ The first inequality was proved by counting paths, and the second by counting circuits in the dual. These approaches are fundamental to proofs of the existence of phase transition in a multitude of settings.

\subsection{A Question}

The definition of $p_{\mathrm{c}}$ entails that

$$
\theta(p) \begin{cases}=0 & \text { if } p<p_{\mathrm{c}}, \\ >0 & \text { if } p>p_{\mathrm{c}},\end{cases}
$$

but what happens when $p=p_{\mathrm{c}}$ ?

Conjecture 3.8. $\theta\left(p_{\mathrm{c}}\right)=0$

This conjecture is known to be valid when $d=2$ (using duality, see Section 9.1 ) and for sufficiently large $d$, currently $d \geq 19$ (using the 'bubble expansion', see Section 8.5). Concentrate your mind on the case $d=3$.

Let us turn to the existence of an infinite open cluster, and set

$$
\psi(p)=P_{p}(|C(x)|=\infty \text { for some } x) .
$$

By using the usual zero-one law (see [170], p. 290), for any $p$ either $\psi(p)=0$ or $\psi(p)=1$. Using the fact that $\mathbb{Z}^{d}$ is countable, we have that

$$
\psi(p)=1 \quad \text { if and only if } \theta(p)>0 .
$$

The above conjecture may therefore be written equivalently as $\psi\left(p_{\mathrm{c}}\right)=0$.

There has been progress towards this conjecture: see $[50,165]$. It is proved that, when $p=p_{\mathrm{c}}$, no half-space of $\mathbb{Z}^{d}$ (where $d>3$ ) can contain an infinite open cluster. Therefore we are asked to eliminate the following absurd possibility: there exist a.s. an infinite open cluster in $\mathbb{L}^{d}$, but any such cluster is a.s. cut into finite parts by the removal of all edges of the form $\langle x, x+e\rangle$, as $x$ ranges over a hyperplane of $L^{d}$ and where $e$ is a unit vector perpendicular to this hyperplane. 


\section{INEQUALITIES FOR CRITICAL PROBABILITIES}

4.1 Russo's Formula

There is a fundamental formula, known in this area as Russo's formula but developed earlier in the context of reliability theory. Let $E$ be a finite set, and let $\Omega_{E}=\{0,1\}^{E}$ For $\omega \in \Omega_{E}$ and $e \in E$, we define the configurations $\omega^{e}, \omega_{e}$ by

$$
\omega^{e}(f)=\left\{\begin{array}{ll}
\omega(f) & \text { if } f \neq e, \\
1 & \text { if } f=e,
\end{array} \quad \omega_{e}(f)= \begin{cases}\omega(f) & \text { if } f \neq e, \\
0 & \text { if } f=e .\end{cases}\right.
$$

Let $A$ be a subset of $\Omega_{E}$, i.e., an event. For $\omega \in \Omega_{E}$, we call $e$ pivotal for $A$ if

$$
\text { either } \omega^{e} \in A, \omega_{e} \notin A \text { or } \omega^{e} \notin A, \omega_{e} \in A,
$$

which is to say that the occurrence or not of $A$ depends on the state of the edge $e$. Note that the set of pivotal edges for $A$ depends on the choice of $\omega$. We write $N_{A}$ for the number of pivotal edges for $A$ (so that $N_{A}$ is a random variable). Finally, let $N: \Omega_{E} \rightarrow \mathbb{R}$ be given by

$$
N(\omega)=\sum_{e \in E} \omega(e),
$$

the 'total number of open edges'

Theorem 4.2. Let $0<p<1$

(a) For any event $A$

$$
\frac{d}{d p} P_{p}(A)=\frac{1}{p(1-p)} \operatorname{cov}_{p}\left(N, 1_{A}\right) .
$$

(b) For any increasing event $A$,

$$
\frac{d}{d p} P_{p}(A)=E_{p}\left(N_{A}\right)
$$

Here, $P_{p}$ and $E_{p}$ are the usual product measure and expectation on $\Omega_{E}$, and $\operatorname{cov}_{p}$ denotes covariance.

Proof. We have that

$$
P_{p}(A)=\sum_{\omega} p^{N(\omega)}(1-p)^{|E|-N(\omega)} 1_{A}(\omega)
$$

whence

$$
\begin{aligned}
\frac{d}{d p} P_{p}(A) & =\sum_{\omega}\left(\frac{N(\omega)}{p}-\frac{|E|-N(\omega)}{1-p}\right) 1_{A}(\omega) P_{p}(\omega) \\
& =\frac{1}{p(1-p)} E_{p}\left(\{N-p|E|\} 1_{A}\right),
\end{aligned}
$$

as required for part (a)

Turning to (b), assume $A$ is increasing. Using the definition of $N$, we have that

$$
\operatorname{cov}_{p}\left(N, 1_{A}\right)=\sum_{e \in E}\left\{P_{p}\left(A \cap J_{e}\right)-p P_{p}(A)\right\}
$$

where $J_{e}=\{\omega(e)=1\}$. Now, writing $\{$ piv $\}$ for the event that $e$ is pivotal for $A$,

$$
P_{p}\left(A \cap J_{e}\right)=P_{p}\left(A \cap J_{e} \cap\{\operatorname{piv}\}\right)+P_{p}\left(A \cap J_{e} \cap\{\text { not piv }\}\right) .
$$

We use the important fact that $J_{e}$ is independent of $\{$ piv $\}$, which holds since the latter event depends only on the states of edges $f$ other than $e$. Since $A \cap J_{e} \cap\{$ piv $\}=$ $J_{e} \cap\{$ piv $\}$, the first term on the right side above equals

$$
P_{p}\left(J_{e} \cap\{\operatorname{piv}\}\right)=P_{p}\left(J_{e} \mid \operatorname{piv}\right) P_{p}(\operatorname{piv})=p P_{p}(\text { piv }),
$$

and similarly the second term equals (since $J_{e}$ is independent of the event $A \cap$ $\{$ not piv $\}$

$$
P_{p}\left(J_{e} \mid A \cap\{\text { not } \operatorname{piv}\}\right) P_{p}(A \cap\{\text { not } \operatorname{piv}\})=p P_{p}(A \cap\{\text { not piv }\})
$$

Returning to (4.3), the summand equals

$$
\begin{aligned}
\left\{p P_{p}(\mathrm{piv})\right. & \left.+p P_{p}(A \cap\{\text { not } \operatorname{piv}\})\right\}-p\left\{P_{p}(A \cap\{\text { piv }\})+P_{p}(A \cap\{\text { not piv }\})\right\} \\
& =p P_{p}(\bar{A} \cap\{\operatorname{piv}\})=p P_{p}\left(\overline{J_{e}} \mid \operatorname{piv}\right) P_{p}(\text { piv }) \\
& =p(1-p) P_{p}(\text { piv }) .
\end{aligned}
$$

Insert this into (4.3) to obtain part (b) from part (a). An alternative proof of part (b) may be found in $[\mathrm{G}]$.

Although the above theorem was given for a finite product space $\Omega_{E}$, the conclusion is clearly valid for the infinite space $\Omega$ so long as the event $A$ is finitedimensional.

The methods above may be used further to obtain formulae for the higher derivatives of $P_{p}(A)$. First, Theorem 4.2(b) may be generalised to obtain that

$$
\frac{d}{d p} E_{p}(X)=\sum_{e \in E} E_{p}\left(\delta_{e} X\right)
$$

where $X$ is any given random variable on $\Omega$ and $\delta_{e} X$ is defined by $\delta_{e} X(\omega)=$ $X\left(\omega^{e}\right)-X\left(\omega_{e}\right)$. It follows that

$$
\frac{d^{2}}{d p^{2}} E_{p}(X)=\sum_{e, f \in E} E_{p}\left(\delta_{e} \delta_{f} X\right) .
$$

Now $\delta_{e} \delta_{e} X=0$, and for $e \neq f$

$$
\delta_{e} \delta_{f} X(\omega)=X\left(\omega^{e f}\right)-X\left(\omega_{f}^{e}\right)-X\left(\omega_{e}^{f}\right)+X\left(\omega_{e f}\right) .
$$


Let $X=1_{A}$ where $A$ is an increasing event. We deduce that

$$
\begin{aligned}
\frac{d^{2}}{d p^{2}} P_{p}(A) & =\sum_{\substack{e, f \in E \\
e \neq f}}\left\{1_{A}\left(\omega^{e f}\right)\left(1-1_{A}\left(\omega_{e}^{f}\right)\right)\left(1-1_{A}\left(\omega_{f}^{e}\right)\right)\right. \\
& =E_{p}\left(N_{A}^{\mathrm{ser}}\right)-E_{p}\left(N_{A}^{\mathrm{par}}\right)
\end{aligned}
$$

where $N_{A}^{\text {ser }}$ (resp. $N^{\mathrm{par}}$ ) is the number of distinct ordered pairs $e, f$ of edges such that $\omega^{e f} \in A$ but $\omega_{e}^{f}, \omega_{f}^{e} \notin A$ (resp. $\omega_{e}^{f}, \omega_{f}^{e} \in A$ but $\omega_{\text {ef }} \notin A$ ). (The superscripts here are abbreviations for 'series' and 'parallel'.) This argument may be generalised to higher derivatives.

4.2 Strict Inequalities for Critical Probabilities

If $\mathcal{L}$ is a sublattice of the lattice $\mathcal{L}^{\prime}$ (written $\mathcal{L} \subset \mathcal{L}^{\prime}$ ) then clearly $p_{\mathrm{c}}(\mathcal{L}) \geq p_{\mathrm{c}}\left(\mathcal{L}^{\prime}\right)$ but when does the strict inequality $p_{\mathrm{c}}(\mathcal{L})>p_{\mathrm{c}}\left(\mathcal{L}^{\prime}\right)$ hold? The question may be quantified by asking for non-trivial lower bounds for $p_{\mathrm{c}}(\mathcal{L})-p_{\mathrm{c}}\left(\mathcal{L}^{\prime}\right)$.

Similar questions arise in many ways, not simply within percolation theory. More generally, consider any process indexed by a continuously varying parameter $T$ and enjoying a phase transition at some point $T=T_{\mathrm{c}}$. In many cases of interest, enough structure is available to enable us to conclude that certain systematic changes to the process can change $T_{\mathrm{c}}$ but that any such change must push $T_{\mathrm{c}}$ in one particular direction (thereby increasing $T_{\mathrm{c}}$, say). The question then is to understand which systematic changes chenge $T_{c}$ strictly. In the context of the previous parag in in not in $\mathcal{L}$

A related percolation question is that of 'entanglements'. Consider bond percolation on $\mathbb{L}^{3}$, and examine the box $B(n)$. Think about the open edges as being solid connections made of elastic, say. Try to 'pull apart' a pair of opposite faces of $B(n)$. If $p>p_{\mathrm{c}}$, then you will generally fail because, with large probability (tending to as $n \rightarrow \infty$ ), there is an open path joining one face to the other. Even if $p<p_{\mathrm{c}}$ then you may fail, owing to an 'entanglement' of open paths (a necklace of necklaces, perhaps, see Figure 411). It may be seen that there is an 'entanglement transition'

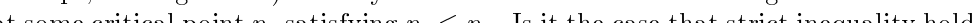
.e, $p_{\mathrm{e}}<p_{\mathrm{c}}$ ?

A technology has been developed for approaching such questions of strict inequality. Although, in particular cases, ad hoc arguments can be successful, there appears to be only one general approach. We illustrate this approach in the next section, by sketching the details in a particular case.

Important references include $[20,157,158,269]$. See also [75].

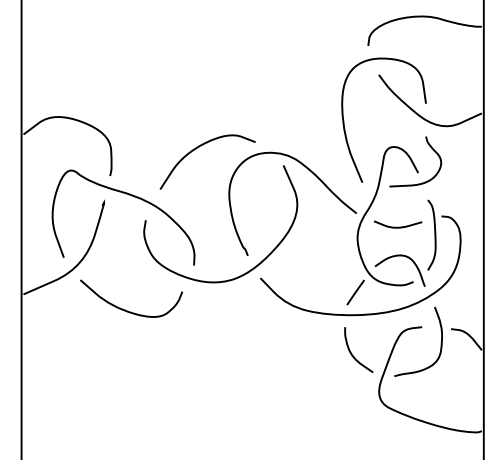

Fig. 4.1. An entanglement between opposite sides of a cube in three dimensions. Note the necklace of necklaces on the right.

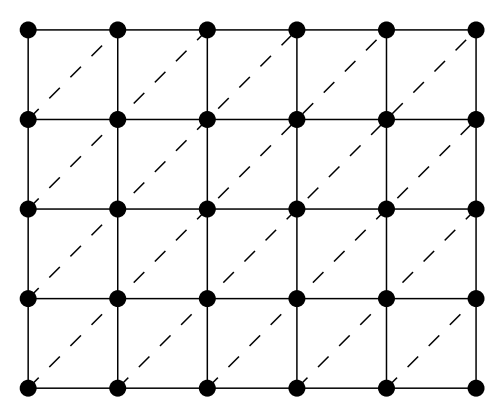

Fig. 4.2. The triangular lattice may be obtained from the square lattice by the addition of certain diagonals

4.3 The Square and Triangular Lattices

The triangular lattice $\mathbb{T}$ may be obtained by adding diagonals across the squares of the square lattice $\mathbb{L}^{2}$, in the manner of Figure 4.2. Since any infinite open cluster of $\mathbb{L}^{2}$ is also an infinite open cluster of $\mathbb{T}$, it follows that $p_{\mathrm{c}}(\mathbb{T}) \leq p_{\mathrm{c}}\left(\mathbb{L}^{2}\right)$, but does strict inequality hold? There are various ways of proving the strict inequality. Here we adopt the canonical argument of [20], as an illustration of a general technique.

Before embarking on this exercise, we point out that, for this particular case, there is a variety of ways of obtaining the result, by using special properties of the square and triangular lattices. The attraction of the method described here is its generality, relying as it does on essentially no assumptions about graph-structure or number of dimensions.

First we embed the problem in a two-parameter system. Let $0 \leq p, s \leq 1$. We declare each edge of $\mathbb{T}^{2}$ to be open with probability $p$, and each further edge of $\mathbb{T}$ 
(i.e., the dashed edges in Figure 4.2) to be open with probability $s$. Writing $P_{p, s}$ for the associated measure, define

$$
\theta(p, s)=P_{p, s}(0 \leftrightarrow \infty) .
$$

We propose to prove differential inequalities which imply that $\partial \theta / \partial p$ and $\partial \theta / \partial s$ are comparable, uniformly on any closed subset of the interior $(0,1)^{2}$ of the parameter space. This cannot itself be literally achieved, since we have insufficient information about the differentiability of $\theta$. Therefore we approximate $\theta$ by a finitevolume quantity $\theta_{n}$, and we then work with the partial derivatives of $\theta_{n}$.

For any set $A$ of vertices, we define the 'interior boundary' $\partial A$ by

$$
\partial A=\{a \in A: a \sim b \text { for some } b \notin A\} .
$$

Let $B(n)=[-n, n]^{d}$, and define

$$
\theta_{n}(p, s)=P_{p, s}(0 \leftrightarrow \partial B(n)) .
$$

Note that $\theta_{n}$ is a polynomial in $p$ and $s$, and that

$$
\theta_{n}(p, s) \downarrow \theta(p, s) \quad \text { as } n \rightarrow \infty \text {. }
$$

Lemma 4.5. There exists a positive integer $L$ and a continuous strictly positive function $g:(0,1)^{2} \rightarrow(0, \infty)$ such that

$$
g(p, s)^{-1} \frac{\partial}{\partial p} \theta_{n}(p, s) \geq \frac{\partial}{\partial s} \theta_{n}(p, s) \geq g(p, s) \frac{\partial}{\partial p} \theta_{n}(p, s)
$$

for $0<p, s<1, n \geq L$

Once this is proved, the main result follows immediately, namely the following. Theorem 4.7. It is the case that $p_{\mathrm{c}}(\mathbb{T})<p_{\mathrm{c}}\left(\mathbb{L}^{2}\right)$.

Sketch Proof of Theorem 4.7. Here is a rough argument, which needs some rigour. There is a 'critical curve' in $(p, s)$-space, separating the regime where $\theta(p, s)=0$ from that when $\theta(p, s)>0$ (see Figure 4.3). Suppose that this critical curve may be written in the form $h(p, s)=0$ for some increasing and continuously differentiable function $h$. It is enough to prove that the graph of $h$ contains no vertical segment. Now

$$
\nabla h=\left(\frac{\partial h}{\partial p}, \frac{\partial h}{\partial s}\right)
$$

and, by Lemma 4.5 ,

$$
\nabla h \cdot(0,1)=\frac{\partial h}{\partial s} \geq g(p, s) \frac{\partial h}{\partial p},
$$

whence

$$
\frac{1}{|\nabla h|} \frac{\partial h}{\partial s}=\left\{\left(\frac{\partial h}{\partial p} / \frac{\partial h}{\partial s}\right)^{2}+1\right\}^{-\frac{1}{2}} \geq \frac{g}{\sqrt{g^{2}+1}},
$$

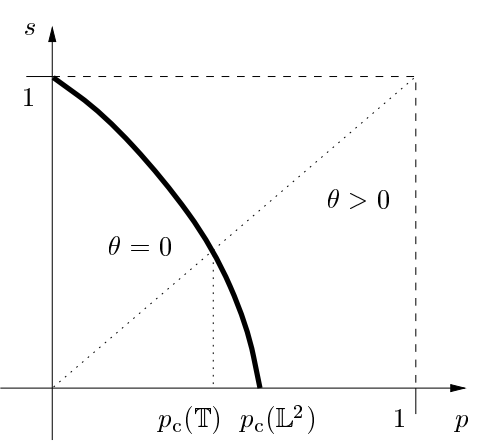

Fig. 4.3. The critical 'surface'. The area beneath the curve is the set of $(p, s)$ for which $\theta(p, s)=0$.

which is bounded away from 0 on any closed subset of $(0,1)^{2}$. This indicates as required that $h$ has no vertical segment.

Here is the proper argument. There is more than one way of defining the critical surface. Let $C_{\mathrm{sub}}=\{(p, s): \theta(p, s)=0\}$, and let $C_{\text {crit }}$ be the set of all points lying in the closure of both $C_{\text {sub }}$ and its complement.

Let $\eta$ be positive and small, and find $\gamma(>0)$ such that $g(p, s) \geq \gamma$ on $[\eta, 1-\eta]^{2}$. At the point $(a, b) \in[\eta, 1-\eta]^{2}$, the rate of change of $\theta_{n}(a, b)$ in the direction $(\cos \alpha,-\sin \alpha)$, where $0 \leq \alpha<\frac{\pi}{2}$, is

$$
\begin{aligned}
\nabla \theta_{n} \cdot(\cos \alpha,-\sin \alpha) & =\frac{\partial \theta_{n}}{\partial a} \cos \alpha-\frac{\partial \theta_{n}}{\partial b} \sin \alpha \\
& \leq \frac{\partial \theta_{n}}{\partial a}(\cos \alpha-\gamma \sin \alpha) \leq 0
\end{aligned}
$$

so long as $\tan \alpha \geq \gamma^{-1}$

Suppose $\theta(a, b)=0$, and $\tan \alpha=\gamma^{-1}$. Let $\left(a^{\prime}, b^{\prime}\right)=(a, b)+\epsilon(\cos \alpha,-\sin \alpha)$ where $\epsilon$ is small and positive. Then, by (4.8),

$$
\theta\left(a^{\prime}, b^{\prime}\right)=\lim _{n \rightarrow \infty} \theta_{n}\left(a^{\prime}, b^{\prime}\right) \leq \lim _{n \rightarrow \infty} \theta_{n}(a, b)=\theta(a, b)=0,
$$

whence $\left(a^{\prime}, b^{\prime}\right) \in C_{\text {sub }}$

There is quite a lot of information in such a calculation, but we abstract a small amount only. Take $a=b=p_{\mathrm{c}}(\mathbb{T})-\zeta$ for some small positive $\zeta$. Then choose $\epsilon$ large enough so that $a^{\prime}>p_{\mathrm{c}}(\mathbb{T})$. The above calculation, for small enough $\zeta$, implies that

$$
\theta\left(a^{\prime}, 0\right) \leq \theta\left(a^{\prime}, b^{\prime}\right)=0,
$$

whence $p_{\mathrm{c}}\left(\mathbb{L}^{2}\right) \geq a^{\prime}>p_{\mathrm{c}}(\mathbb{T})$ 


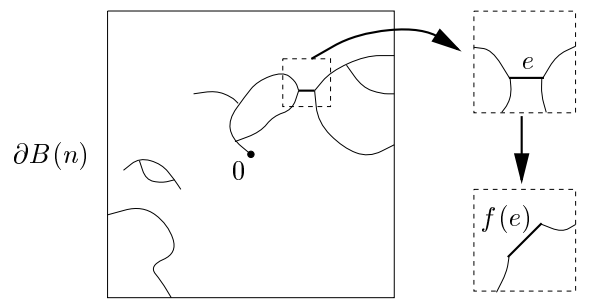

Fig. 4.4. Inside the box $B(n)$, the edge $e$ is pivotal for the event $\{0 \leftrightarrow \partial B(n)\}$. By altering the configuration inside the smaller box, we may construct a configuration in which $f(e)$ is pivotal instead.

Proof of Lemma 4.5. With $\mathbb{E}^{2}$ the edge set of $\mathbb{L}^{2}$, and $\mathbb{F}$ the additional edges in the triangular lattice $\mathbb{T}$ (i.e., the diagonals in Figure 4.2), we have by Russo's formula (in a slightly more general version than Theorem 4.2) that

$$
\begin{aligned}
\frac{\partial}{\partial p} \theta_{n}(p, s) & =\sum_{e \in \mathbb{E}^{2}} P_{p, s}\left(e \text { is pivotal for } A_{n}\right), \\
\frac{\partial}{\partial s} \theta_{n}(p, s) & =\sum_{f \in \mathbb{F}} P_{p, s}\left(f \text { is pivotal for } A_{n}\right),
\end{aligned}
$$

where $A_{n}=\{0 \leftrightarrow \partial B(n)\}$. The idea now is to show that each summand in the first summation is comparable with some given summand in the second. Actually we shall only prove the second inequality in (4.6), since this is the only one used in proving the theorem, and additionally the proof of the other part is similar.

With each edge $e$ of $\mathbb{E}^{2}$ we associate a unique edge $f=f(e)$ of $\mathbb{F}$ such that $f$ lies near to $e$. This may be done in a variety of ways, but in order to be concrete we specify that if $e=\left\langle u, u+e_{1}\right\rangle$ or $e=\left\langle u, u+e_{2}\right\rangle$ then $f=\left\langle u, u+e_{1}+e_{2}\right\rangle$, where $e_{1}$ and $e_{2}$ are unit vectors in the directions of the (increasing) $x$ and $y$ axes.

We claim that there exists a function $h(p, s)$, strictly positive on $(0,1)^{2}$, such that

(4.10) $\quad h(p, s) P_{p, s}\left(e\right.$ is pivotal for $\left.A_{n}\right) \leq P_{p, s}\left(f(e)\right.$ is pivotal for $\left.A_{n}\right)$

for all $e$ lying in $B(n)$. Once this is shown, we sum over $e$ to obtain by (4.9) that

$$
\begin{aligned}
h(p, s) \frac{\partial}{\partial p} \theta_{n}(p, s) & \leq \sum_{e \in \mathbb{E}^{2}} P_{p, s}\left(f(e) \text { is pivotal for } A_{n}\right) \\
& \leq 2 \sum_{f \in \mathbb{F}} P_{p, s}\left(f \text { is pivotal for } A_{n}\right) \\
& =2 \frac{\partial}{\partial s} \theta_{n}(p, s)
\end{aligned}
$$

as required. The factor 2 arises because, for each $f(\in \mathbb{F})$, there are exactly two edges $e$ with $f(e)=f$.
Finally, we indicate the reason for (4.10). Let us consider the event $\{e$ is pivotal for $\left.A_{n}\right\}$. We claim that there exists an integer $M$, chosen uniformly for edges $e$ in $B(n)$ and for all large $n$, such that

(a) all paths from 0 to $\partial B(n)$ pass through the region $e+B(M)$

(b) by altering the configuration within $e+B(M)$ only, we may obtain an event on which $f(e)$ is pivotal for $A_{n}$.

This claim is proved by inspecting Figure 4.4. A special argument may be needed when the box $e+B(M)$ either contains the origin or intersects $\partial B(n)$, but such special arguments pose no substantial difficulty. Once this geometrical claim is accepted, (4.10) follows thus. Write $E_{g}$ for the event that the edge $g$ is pivotal for $A_{n}$. For $\omega \in E_{e}$, let $\omega^{\prime}=\omega^{\prime}(\omega)$ be the configuration obtained as above, so that $\omega^{\prime}$ agrees with $\omega$ off $e+B(M)$, and furthermore $\omega^{\prime} \in E_{f(e)}$. Then

$$
P_{p, s}\left(E_{e}\right)=\sum_{\omega \in E_{e}} P_{p, s}(\omega) \leq \sum_{\omega \in E_{e}} \frac{1}{\alpha^{R}} P_{p, s}\left(\omega^{\prime}\right) \leq\left(\frac{2}{\alpha}\right)^{R} P_{p, s}\left(E_{f(e)}\right)
$$

where $\alpha=\min \{p, s, 1-p, 1-s\}$ and $R$ is the number of edges of $\mathbb{T}$ in $e+B(M)$.

\subsection{ENHANCEMENTS}

An 'enhancement' is loosely defined as a systematic addition of connections according to local rules. Enhancements may involve further coin flips. Can an enhancement create an infinite cluster when previously there was none?

Clearly the answer can be negative. For example the rule may be of the type: join any two neighbours of $\mathbb{Z}^{d}$ with probability $\frac{1}{2} p_{\mathrm{c}}$, whenever they have no incident open edges. Such an enhancement creates extra connections but (a.s.) no extra infinite cluster.

Here is a proper definition. Consider bond percolation on $\mathbb{L}^{d}$ with parameter $p$, and consider enhancements of the following type. Let $R>0$, and let $f$ be a function which associates to each configuration on the box $B(R)$ a graph on $\mathbb{Z}^{d}$ with finitely many edges. For each $x \in \mathbb{Z}^{d}$, we observe the configuration $\omega$ on the box $x+B(R)$, and we write $f(x, \omega)$ for the associated evaluation of $f$. The enhanced configuration is the graph

$$
G(\mathrm{enh})=G(\omega) \cup\left\{\bigcup_{x: H(x)=1}\{x+f(x, \omega)\}\right\}
$$

where $G(\omega)$ is the graph of open edges, and $\left\{H(x): x \in \mathbb{Z}^{d}\right\}$ is a family of Bernoulli random variables, each taking the value 1 with probability $s$ (independently of everything else). The parameter $s$ is the 'density' of the enhancement. In writing the union of graphs, we mean the graph with vertex set $\mathbb{Z}^{d}$ having the union of the appropriate edge sets.

We call such an enhancement essential if there exists a percolation configuration $\omega$ containing no doubly-infinite open path but such that $G(\omega) \cup f(0, \omega)$ does contain such a path. The following theorem is taken from [20] and may be proved in a manner similar to the proof given in the last section. 
25

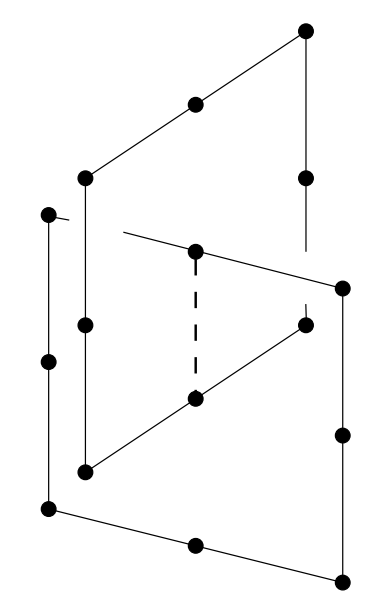

Fig 4.5. A sketch of the enhancement which adds an edge between any two interlocking $2 \times 2$ squares in $\mathbb{L}^{3}$.

Theorem 4.11. Let $s>0$. For any essential enhancement, there exists a nonempty interval $\left(\pi(s), p_{\mathrm{c}}\right)$ such that

$$
P(G(\mathrm{enh}) \text { contains an infinite cluster })>0
$$

when $\pi(s)<p \leq p_{\mathrm{c}}$

That is, essential enhancements shift the critical point strictly. Here is such an enhancement relevant to the entanglement transition in $\mathbb{L}^{3}$. Whenever we see two interlinking $2 \times 2$ open squares, then we join them by an edge (see Figure 4.5). It is easy to see that this enhancement is essential, and therefore it shifts the critical point downwards. Hence the entanglement critical point $p_{\mathrm{e}}$ satisfies $p_{\mathrm{e}}<p_{\mathrm{c}}$. See $[20,198]$.

Finally we note that one may find explicit functions $g$ in Lemma 4.5, whence the mechanism of the method leads to numerical lower bounds on the change in critical value.

\section{CORRELATION INEQUALITIES}

\subsection{FKG INEQUALITY}

The FKG inequality for percolation processes was discovered by Harris [181], and is often named now after the authors of [144] who proved a more general version which is the subject of this section.

Let $E$ be a finite set, and $\Omega_{E}=\{0,1\}^{E}$ as usual. We write $\mathcal{F}_{E}$ for the set of all subsets of $\Omega_{E}$, and call a probability measure $\mu$ on $\left(\Omega_{E}, \mathcal{F}_{E}\right)$ positive if $\mu(\omega)>0$ for all $\omega \in \Omega_{E}$.

Theorem 5.1 (FKG Inequality). Let $\mu$ be a positive probability measure on $\left(\Omega_{E}, \mathcal{F}_{E}\right)$ such that

(5.2) $\quad \mu\left(\omega_{1} \vee \omega_{2}\right) \mu\left(\omega_{1} \wedge \omega_{2}\right) \geq \mu\left(\omega_{1}\right) \mu\left(\omega_{2}\right) \quad$ for all $\omega_{1}, \omega_{2} \in \Omega_{E}$.

Then

$$
\mu(f g) \geq \mu(f) \mu(g)
$$

for all increasing random variables $f, g: \Omega_{E} \rightarrow \mathbb{R}$.

Here, $\omega_{1} \vee \omega_{2}$ and $\omega_{1} \wedge \omega_{2}$ are defined as the maximum and minimum configurations,

$$
\omega_{1} \vee \omega_{2}(e)=\max \left\{\omega_{1}(e), \omega_{2}(e)\right\}, \quad \omega_{1} \wedge \omega_{2}(e)=\min \left\{\omega_{1}(e), \omega_{2}(e)\right\},
$$

for all $e \in E$. In (5.3), we have used $\mu$ to denote expectation as well as probability. Specialising to the indicator functions $f=1_{A}, g=1_{B}$, inequality (5.3) implies that

$$
\mu(A \cap B) \geq \mu(A) \mu(B) \quad \text { for increasing events } A, B .
$$

It is easily checked that the product measure $P_{p}$ satisfies the hypotheses of the theorem (when $0<p<1$ ), and therefore $P_{p}$ satisfies the FKG inequality (5.3). This inequality may be proved directly in the special case of product measure (see $[\mathrm{G}]$, p. 26). Here we shall prove the more general theorem given above. The proof proceeds by first proving a theorem about stochastic orderings of measures, usually called Holley's inequality after [192].

Theorem 5.5 (Holley's Inequality). Let $\mu_{1}$ and $\mu_{2}$ be positive probability measures on $\left(\Omega_{E}, \mathcal{F}_{E}\right)$ such that

(5.6) $\quad \mu_{1}\left(\omega_{1} \vee \omega_{2}\right) \mu_{2}\left(\omega_{1} \wedge \omega_{2}\right) \geq \mu_{1}\left(\omega_{1}\right) \mu_{2}\left(\omega_{2}\right) \quad$ for all $\omega_{1}, \omega_{2} \in \Omega_{E}$.

Then

$$
\mu_{1}(f) \geq \mu_{2}(f) \quad \text { for all increasing } f: \Omega_{E} \rightarrow \mathbb{R}
$$


which is to say that $\mu_{1} \geq \mu_{2}$.

Proof of Theorem 5.5. The theorem is 'merely' a numerical inequality involving a finite number of positive reals. It may be proved in a totally elementary manner, using essentially no general mechanism. Nevertheless, in a more useful (and remarkable) proof we construct Markov chains and appeal to the ergodic theorem. This requires a mechanism, but the method is beautiful, and in addition yields a structure which finds applications elsewhere.

The main step is the proof that $\mu_{1}$ and $\mu_{2}$ can be 'coupled' in such a way that the component with marginal measure $\mu_{1}$ lies above (in the sense of sample realisations) that with marginal measure $\mu_{2}$. This is achieved by constructing a certain Markov chain with the coupled measure as unique invariant measure.

Here is a preliminary calculation. Let $\mu$ be a positive probability measure on $\left(\Omega_{E}, \mathcal{F}_{E}\right)$. We may construct a time-reversible Markov chain with state space $\Omega_{E}$ and unique invariant measure $\mu$, in the following way. We do this by choosing a suitable generator (or ' $Q$-matrix') satisfying the detailed balance equations. The dynamics of the chain involve the 'switching on or off' of components of the current state. For $\omega \in \Omega_{E}$, let $\omega^{e}$ and $\omega_{e}$ be given as in (4.1). Define the function $G: \Omega_{E}^{2} \rightarrow$ $\mathbb{R}$ by

$$
G\left(\omega_{e}, \omega^{e}\right)=1, \quad G\left(\omega^{e}, \omega_{e}\right)=\frac{\mu\left(\omega_{e}\right)}{\mu\left(\omega^{e}\right)},
$$

for all $\omega \in \Omega_{E}, e \in E$; define $G\left(\omega, \omega^{\prime}\right)=0$ for all other pairs $\omega, \omega^{\prime}$ with $\omega \neq \omega^{\prime}$. The diagonal elements are chosen so that

$$
\sum_{\omega^{\prime}} G\left(\omega, \omega^{\prime}\right)=0 \quad \text { for all } \omega \in \Omega_{E} .
$$

It is elementary that

$$
\mu(\omega) G\left(\omega, \omega^{\prime}\right)=\mu\left(\omega^{\prime}\right) G\left(\omega^{\prime}, \omega\right) \quad \text { for all } \omega, \omega^{\prime} \in \Omega_{E},
$$

and therefore $G$ generates a time-reversible Markov chain on the state space $\Omega_{E}$. This chain is irreducible (using (5.7)), and therefore has a unique invariant measure $\mu$ (see [170], p. 208).

We next follow a similar route for pairs of configurations. Let $\mu_{1}$ and $\mu_{2}$ satisfy the hypotheses of the theorem, and let $S$ be the set of all pairs $(\pi, \omega)$ of configurations in $\Omega_{E}$ satisfying $\pi \leq \omega$. We define $H: S \times S \rightarrow \mathbb{R}$ by

$$
\begin{aligned}
H\left(\pi_{e}, \omega ; \pi^{e}, \omega^{e}\right) & =1, \\
H\left(\pi, \omega^{e} ; \pi_{e}, \omega_{e}\right) & =\frac{\mu_{1}\left(\omega_{e}\right)}{\mu_{1}\left(\omega^{e}\right)}, \\
H\left(\pi^{e}, \omega^{e} ; \pi_{e}, \omega^{e}\right) & =\frac{\mu_{2}\left(\pi_{e}\right)}{\mu_{2}\left(\pi^{e}\right)}-\frac{\mu_{1}\left(\omega_{e}\right)}{\mu_{1}\left(\omega^{e}\right)},
\end{aligned}
$$

for all $(\pi, \omega) \in S$ and $e \in E$; all other off-diagonal values of $H$ are set to 0 . The diagonal terms are chosen so that

$$
\sum_{\pi^{\prime}, \omega^{\prime}} H\left(\pi, \omega ; \pi^{\prime}, \omega^{\prime}\right)=0 \quad \text { for all }(\pi, \omega) \in S
$$

Equation (5.8) specifies that, for $\pi \in \Omega_{E}$ and $e \in E$, the edge $e$ is acquired by $\pi$ (if it does not already contain it) at rate 1 ; any edge so acquired is added also to $\omega$ if it does not already contain it. (Here, we speak of a configuration $\psi$ containing an edge $e$ if $\psi(e)=1$.) Equation (5.9) specifies that, for $\omega \in \Omega_{E}$ and $e \in E$ with $\omega(e)=1$, the edge $e$ is removed from $\omega$ (and also from $\pi$ if $\pi(e)=1$ ) at the rate given in (5.9). For $e$ with $\pi(e)=1$, there is an additional rate given in (5.10) at which $e$ is removed from $\pi$ but not from $\omega$. We need to check that this additiona rate is indeed non-negative. This poses no problem, since the required inequality

$$
\mu_{1}\left(\omega^{e}\right) \mu_{2}\left(\pi_{e}\right) \geq \mu_{1}\left(\omega_{e}\right) \mu_{2}\left(\pi^{e}\right) \quad \text { where } \pi \leq \omega
$$

\section{follows from assumption (5.6)}

Let $\left(X_{t}, Y_{t}\right)_{t>0}$ be a Markov chain on $S$ having generator $H$, and set $\left(X_{0}, Y_{0}\right)=$ $(0,1)$, where 0 (resp. 1) is the state of all 0 's (resp. 1's). By examination of (5.8)(5.10) we see that $X=\left(X_{t}\right)_{t \geq 0}$ is a Markov chain with generator given by (5.7) with $\mu=\mu_{2}$, and that $Y=\left(Y_{t}\right)_{t \geq 0}$ arises similarly with $\mu=\mu_{1}$.

Let $\kappa$ be an invariant measure for the paired chain $\left(X_{t}, Y_{t}\right)_{t \geq 0}$. Since $X$ and $Y$ have (respective) unique invariant measures $\mu_{2}$ and $\mu_{1}$, it follows that the marginals of $\kappa$ are $\mu_{2}$ and $\mu_{1}$. We have by construction that

$$
\kappa(\{(\pi, \omega): \pi \leq \omega\})=1,
$$

and $\kappa$ is the required 'coupling' of $\mu_{1}$ and $\mu_{2}$.

Let $(\pi, \omega) \in S$ be chosen according to the measure $\kappa$. Then

$$
\mu_{1}(f)=\kappa(f(\omega)) \geq \kappa(f(\pi))=\mu_{2}(f),
$$

for any increasing function $f$. Therefore $\mu_{1} \geq \mu_{2}$.

Proof of Theorem 5.1. Assume that $\mu$ satisfies (5.2), and let $f$ and $g$ be increasing functions. By adding a constant to the function $g$, we see that it suffices to prove (5.3) under the extra hypothesis that $g$ is strictly positive. We assume this holds. Define positive probability measures $\mu_{1}$ and $\mu_{2}$ on $\left(\Omega_{E}, \mathcal{F}_{E}\right)$ by $\mu_{2}=\mu$ and

$$
\mu_{1}(\omega)=\frac{g(\omega) \mu(\omega)}{\sum_{\omega^{\prime}} g\left(\omega^{\prime}\right) \mu\left(\omega^{\prime}\right)} \quad \text { for } \omega \in \Omega_{E} .
$$

Since $g$ is increasing, (5.6) follows from (5.2). By Holley's inequality,

$$
\mu_{1}(f) \geq \mu_{2}(f)
$$

which is to say that

$$
\frac{\sum_{\omega} f(\omega) g(\omega) \mu(\omega)}{\sum_{\omega^{\prime}} g\left(\omega^{\prime}\right) \mu\left(\omega^{\prime}\right)} \geq \sum_{\omega} f(\omega) \mu(\omega)
$$

as required. 


\subsection{DisJoint OCCURRENCE}

Van den Berg has suggested a converse to the FKG inequality, namely that, for some interpretation of the binary operation $\circ$,

$$
P_{p}(A \circ B) \leq P_{p}(A) P_{p}(B) \text { for all increasing events } A, B \text {. }
$$

The correct interpretation of $A \circ B$ turns out to be ' $A$ and $B$ occur disjointly'. We explain this statement next

As usual, $E$ is a finite set, $\Omega_{E}=\{0,1\}^{E}$, and so on. For $\omega \in \Omega_{E}$, let

$$
K(\omega)=\{e \in E: \omega(e)=1\},
$$

so that there is a one-one correspondence between configurations $\omega$ and sets $K(\omega)$. For increasing events $A, B$, let

$$
\begin{gathered}
A \circ B=\left\{\omega: \text { for some } H \subseteq K(\omega), \text { we have that } \omega^{\prime} \in A \text { and } \omega^{\prime \prime} \in B,\right. \\
\text { where } \left.K\left(\omega^{\prime}\right)=H \text { and } K\left(\omega^{\prime \prime}\right)=K(\omega) \backslash H\right\},
\end{gathered}
$$

and we call $A \circ B$ the event that $A$ and $B$ occur disjointly.

The canonical example of disjoint occurrence in percolation theory concerns the existence of disjoint open paths. If $A=\{u \leftrightarrow v\}$ and $B=\{x \leftrightarrow y\}$, then $A \circ B$ is the event that are two edge-disjoint paths, one joining $u$ to $v$, and the other joining $x$ to $y$.

Theorem 5.11 (BK Inequality [67]). If $A$ and $B$ are increasing events, then

$$
P_{p}(A \circ B) \leq P_{p}(A) P_{p}(B) .
$$

Proof. The following sketch can be made rigorous (see [58], and [G], p. 32). For the sake of being concrete, we take $E$ to be the edge-set of a finite graph $G$, and consider the case when $A=\{u \leftrightarrow v\}$ and $B=\{x \leftrightarrow y\}$ for four distinct vertices $u, v, x, y$.

Let $e$ be an edge of $E$. In the process of 'splitting' $e$, we replace $e$ by two copies $e^{\prime}$ and $e^{\prime \prime}$ of itself, each of which is open with probability $p$ (independently of the other, and of all other edges). Having split $e$, we look for disjoint paths from $u$ to $v$, and from $x$ to $y$, but with the following difference: the path from $u$ to $v$ is not permitted to use $e^{\prime \prime}$, and the path from $x$ to $y$ is not permitted to use $e^{\prime}$.

The crucial observation is that this splitting cannot decrease the chance of finding the required open paths.

We split each edge in turn, the appropriate probability being non-decreasing at each stage. After every edge has been split, we are then looking for two paths within two independent copies of $G$, and this probability is just $P_{p}(A) P_{p}(B)$. Therefore

$$
P_{p}(A \circ B) \leq \cdots \leq P_{p}(A) P_{p}(B) .
$$

Van den Berg and Kesten [67] conjectured a similar inequality for arbitrary $A$ and $B$ (not just the monotone events), with a suitable redefinition of the operation
○. Their conjecture rebutted many serious attempts at proof, before 1995. Here is the more general statement.

For $\omega \in \Omega_{E}, K \subseteq E$, define the cylinder event

$$
C(\omega, K)=\left\{\omega^{\prime}: \omega^{\prime}(e)=\omega(e) \text { for } e \in K\right\} .
$$

Now, for events $A$ and $B$, define

$$
A \square B=\{\omega \text { : for some } K \subseteq E \text {, we have } C(\omega, K) \subseteq A \text { and } C(\omega, \bar{K}) \subseteq B\} .
$$

Theorem 5.12 (Reimer's Inequality [322]). For all events $A$ and $B$,

$$
P_{p}(A \square B) \leq P_{p}(A) P_{p}(B) .
$$

The search is on for 'essential' applications of this beautiful inequality; such an application may be found in the study of dependent percolation models [65]. Related results may be found in $[62,64]$.

Note that Reimer's inequality contains the FKG inequality, by using the fact that $A \square \bar{B}=A \cap \bar{B}$ if $A$ and $B$ are increasing events.

5.3 Site and Bond Percolation

Let $G=(V, E)$ be an infinite connected graph with maximum vertex degree $\Delta$. For a vertex $x$, define $\theta(p, x$, bond) (resp. $\theta(p, x$, site $))$ to be the probability that $x$ lies in an infinite open cluster of $G$ in a bond percolation (resp. site percolation) process on $G$ with parameter $p$. Clearly $\theta(p, x$, bond) and $\theta(p, x$, site) are non-decreasing in p. Also, using the FKG inequality,

$$
\theta(p, x \text {, bond }) \geq P_{p}(\{x \leftrightarrow y\} \cap\{y \leftrightarrow \infty\}) \geq P_{p}(x \leftrightarrow y) \theta(p, y, \text { bond }),
$$

with a similar inequality for the site process. It follows that the critical points

$$
\begin{aligned}
p_{\mathrm{c}}(\text { bond }) & =\sup \{p: \theta(p, x, \text { bond })=0\}, \\
p_{\mathrm{c}}(\text { site }) & =\sup \{p: \theta(p, x, \text { site })=0\},
\end{aligned}
$$

exist and are independent of the choice of the vertex $x$

Theorem 5.13. We have that

$$
\frac{1}{\Delta-1} \leq p_{\mathrm{c}}(\text { bond }) \leq p_{\mathrm{c}}(\text { site }) \leq 1-\left(1-p_{\mathrm{c}}(\text { bond })\right)^{\Delta} .
$$

One consequence of this theorem is that $p_{\mathrm{c}}$ (bond) $<1$ if and only if $p_{\mathrm{c}}($ site $)<1$. The third inequality of (5.14) may be improved by replacing the exponent $\Delta$ by $\Delta-1$, but we do no prove this here. Also, the methods of Chapter 4 may be used to establish the strict inequality $p_{\mathrm{c}}$ (bond) $<p_{\mathrm{c}}($ site). See [169] for proofs of the latter facts. 
Proof. The first inequality of (5.14) follows by counting paths, as in the proof of (3.4). We turn to the remaining two inequalities. Let 0 be a vertex of $G$, called the origin. We claim that

$$
C^{\prime}(p, 0, \text { site }) \leq C(p, 0, \text { bond })
$$

and

$$
C(p, 0, \text { bond }) \leq C^{\prime}\left(p^{\prime}, 0, \text { site }\right) \quad \text { if } p^{\prime} \geq 1-(1-p)^{\Delta},
$$

where " $\leq$ " denotes stochastic ordering, and where $C(p, 0$, bond $)\left(\right.$ resp. $C^{\prime}(p, 0$, site $\left.)\right)$ has the law of the cluster of bond percolation at the origin (resp. the cluster of site percolation at the origin conditional on 0 being an open site). Since

$$
\theta(p, 0, \text { bond })=\operatorname{Prob}(\mid C(p, 0, \text { bond }) \mid=\infty),
$$$$
p^{-1} \theta(p, 0, \text { site })=\operatorname{Prob}\left(\mid C^{\prime}(p, 0, \text { site }) \mid=\infty\right)
$$

the remaining claims of (5.14) follow from (5.15)-(5.16).

We construct appropriate couplings in order to prove (5.15)-(5.16). Let $\omega \in$ $\{0,1\}^{E}$ be a realisation of a bond percolation process on $G=(V, E)$ with density $p$. We may build the cluster at the origin in the following standard manner. Let $e_{1}, e_{2}, \ldots$ be a fixed ordering of $E$. At each stage $k$ of the inductive construction, we shall have a pair $\left(A_{k}, B_{k}\right)$ where $A_{k} \subseteq V, B_{k} \subseteq E$. Initially we set $A_{0}=\{0\}$, $B_{0}=\varnothing$. Having found $\left(A_{k}, B_{k}\right)$ for some $k$, we define $\left(A_{k+1}, B_{k+1}\right)$ as follows. We find the earliest edge $e_{i}$ in the ordering of $E$ with the following properties: $e_{i} \notin B_{k}$, and $e_{i}$ is incident with exactly one vertex of $A_{k}$, say the vertex $x$. We now set

$$
\begin{aligned}
& A_{k+1}= \begin{cases}A_{k} & \text { if } e_{i} \text { is closed, } \\
A_{k} \cup\{y\} & \text { if } e_{i} \text { is open, }\end{cases} \\
& B_{k+1}= \begin{cases}B_{k} \cup\left\{e_{i}\right\} & \text { if } e_{i} \text { is closed, } \\
B_{k} & \text { if } e_{i} \text { is open, }\end{cases}
\end{aligned}
$$

where $e_{i}=\langle x, y\rangle$. If no such edge $e_{i}$ exists, we declare $\left(A_{k+1}, B_{k+1}\right)=\left(A_{k}, B_{k}\right)$. The sets $A_{k}, B_{k}$ are non-decreasing, and the open cluster at the origin is given by $A_{\infty}=\lim _{k \rightarrow \infty} A_{k}$

We now augment the above construction in the following way. We colour the vertex 0 red. Furthermore, on obtaining the edge $e_{i}$ given above, we colour the vertex $y$ red if $e_{i}$ is open, and black otherwise. We specify that each vertex is coloured at most once in the construction, in the sense that any vertex $y$ which is obtained at two or more stages is coloured in perpetuity according to the first colour it receives.

Let $A_{\infty}$ (red) be the set of points connected to the origin by red paths of $G$. It may be seen that $A_{\infty}$ (red) $\subseteq A_{\infty}$, and that $A_{\infty}$ (red) has the same distribution as $C^{\prime}(p, 0$, site). Inequality (5.15) follows.

The derivation of (5.16) is similar but slightly more complicated. We start with a directed version of $G$, namely $\vec{G}=(V, \vec{E})$ obtained from $G$ by replacing each edge $e=\langle x, y\rangle$ by two directed edges, one in each direction, and denoted respectively by $[x, y\rangle$ and $[y, x\rangle$. We now let $\vec{\omega} \in\{0,1\}^{\vec{E}}$ be a realisation of an (oriented) bond percolation process on $\vec{G}$ with density $p$

We colour the origin green. We colour a vertex $x(\neq 0)$ green if at least one edge $f$ of the form $[y, x\rangle$ satisfies $\vec{\omega}(f)=1$; otherwise we colour $x$ black. Then

$$
P_{p}(x \text { is green })=1-(1-p)^{\rho(x)} \leq 1-(1-p)^{\Delta},
$$

where $\rho(x)$ is the degree of $x$, and $\Delta=\max _{x} \rho(x)$.

We now build a copy $A_{\infty}$ of $C(p, 0$, bond $)$ more or less as described above in (5.17)-(5.18). The only difference is that, on obtaining the edge $e_{i}=\langle x, y\rangle$ where $x \in A_{k}, y \notin A_{k}$, we declare $e_{i}$ to be open for the purpose of (5.17)-(5.18) if and only if $\vec{\omega}([x, y\rangle)=1$. Finally, we set $A_{\infty}$ (green) to be the set of points connected to the origin by green paths. It may be seen that $A_{\infty}$ (green) $\supseteq A_{\infty}$ Furthermore, by (5.19), $A_{\infty}$ (green) is no larger in distribution that $C^{\prime}\left(p^{\prime}, 0\right.$, site) where $p^{\prime}=1-(1-p)^{\Delta}$. Inequality (5.16) follows. 


\section{SUBCRITICAL PERCOLATION}

6.1 Using SubadDitivity

We assume throughout this chapter that $p<p_{\mathrm{c}}$. All open clusters are a.s. finite and the phase is sometimes called 'disordered' by mathematical physicists, since there are no long-range connections. In understanding the phase, we need to know how fast the tails of certain distributions go to zero, and a rule of thumb is that 'everything reasonable' should have exponentially decaying tails. In particular, the limits

$$
\begin{aligned}
& \phi(p)=\lim _{n \rightarrow \infty}\left\{-\frac{1}{n} \log P_{p}(0 \leftrightarrow \partial B(n))\right\}, \\
& \zeta(p)=\lim _{n \rightarrow \infty}\left\{-\frac{1}{n} \log P_{p}(|C|=n)\right\},
\end{aligned}
$$

should exist, and be strictly positive when $p<p_{\mathrm{c}}$. The function $\phi(p)$ measures a 'distance effect' and $\zeta(p)$ a 'volume effect'.

The existence of such limits is a quite different matter from their positiveness. Existence is usually proved by an appeal to subadditivity (see below) via a correlation inequality. To show positiveness usually requires a hard estimate.

Theorem 6.1 (Subadditive Inequality). If $\left(x_{r}: r \geq 1\right)$ is a sequence of reals satisfying the subadditive inequality

$$
x_{m+n} \leq x_{m}+x_{n} \text { for all } m, n,
$$

then the limit

$$
\lambda=\lim _{r \rightarrow \infty}\left\{\frac{1}{r} x_{r}\right\}
$$

exists, with $-\infty \leq \lambda<\infty$, and satisfies

$$
\lambda=\inf \left\{\frac{1}{r} x_{r}: r \geq 1\right\} .
$$

The history here is that the existence of exponents such as $\phi(p)$ and $\zeta(p)$ was shown using the subadditive inequality, and their positiveness was obtained under extra hypotheses. These extra hypotheses were then shown to be implied by the assumption $p<p_{\mathrm{c}}$, in important papers of Aizenman and Barsky [13] and Menshikov [268, 271]. The case $d=2$ had been dealt with earlier by Kesten [200, 202].

As an example of the subadditive inequality in action, we present a proof of the existence of $\phi(p)$ (and other things ...). The required 'hard estimate' is given in the next section. We denote by $e_{1}$ a unit vector in the direction of increasing first coordinate.
Theorem 6.2. Let $0<p<1$. The limits

$$
\begin{aligned}
& \phi_{1}(p)=\lim _{n \rightarrow \infty}\left\{-\frac{1}{n} \log P_{p}(0 \leftrightarrow \partial B(n))\right\}, \\
& \phi_{2}(p)=\lim _{n \rightarrow \infty}\left\{-\frac{1}{n} \log P_{p}\left(0 \leftrightarrow n e_{1}\right)\right\},
\end{aligned}
$$

exist and are equal.

Before proving this theorem, we introduce the important concept of 'correlation length'. Suppose that $p<p_{\mathrm{c}}$. In the next section, we shall see that the common limit $\phi(p)$ in (6.3)-(6.4) is strictly positive (whereas it equals 0 when $p \geq p_{\mathrm{c}}$ ). At a basic mathematical level, we define the subcritical correlation length $\xi(p)$ by

$$
\xi(p)=1 / \phi(p) \text { for } p<p_{\mathrm{c}} .
$$

The physical motivation for this definition may be expressed as follows. We begin with the following statistical question. Given certain information about the existence (or not) of long open paths in the lattice, how may we distinguish between the two hypotheses that $p=p_{\mathrm{c}}$ and that $p<p_{\mathrm{c}}$. In particular, on what 'length-scale' need we observe the process in order to distinguish these two possibilities? In order to be concrete, let us suppose that we are told that the event $A_{n}=\{0 \leftrightarrow \partial B(n)\}$ occurs. concrete, let us suppose that we are told that the event $A_{n}=\{0 \leftrightarrow \partial B(n)\}$ occurs.
How large must $n$ be that this information be helpful? In performing the classical How large must $n$ be that this information be helpful? In performing the classical
statistical hypothesis test of $\mathrm{H}_{0}: p=p_{\mathrm{c}}$ versus $\mathrm{H}_{1}: p=p^{\prime}$, where $p^{\prime}<p_{\mathrm{c}}$, we will reject the null hypothesis if

$$
P_{p^{\prime}}\left(A_{n}\right)>\beta P_{p_{\mathrm{c}}}\left(A_{n}\right)
$$

where $\beta(<1)$ is chosen in order to adjust the significance level of the test.

Now $P_{p}\left(A_{n}\right)$ is 'approximately' $e^{-n \phi(p)}$, and we shall see in the next section that $\phi(p)>0$ if and only if $p<p_{\mathrm{c}}$. (The fact that $\phi\left(p_{\mathrm{c}}\right)=0$ is slightly delicate; see [G], equation (5.18).) Inequality (6.6) may therefore be written as $n \phi\left(p^{\prime}\right)<$ $\mathrm{O}(1)$, which is to say that $n$ should be of no greater order than $\xi\left(p^{\prime}\right)=1 / \phi\left(p^{\prime}\right)$. This statistical discussion supports the loosely phrased statement that 'in order to distinguish between bond percolation at $p=p_{\mathrm{c}}$ and at $p=p^{\prime}$, it is necessary to distinguish between bond percolation at $p=p_{\mathrm{c}}$ and at
observe the process over a length-scale of at least $\xi\left(p^{\prime}\right)^{\prime}$.

The existence of the function $\phi$ in Theorem 6.2 will be shown using standard results associated with the subadditive inequality. When such inequalities are explored carefully (see [G], Chapter 5), they yield some smoothness of $\phi$, namely that $\phi$ is continuous and non-increasing on $(0,1]$, and furthermore that $\phi\left(p_{\mathrm{c}}\right)=0$. Taken together with the fact that $\chi(p) \geq \phi(p)^{-1}$ (see [27, G]), we obtain that

$$
\chi\left(p_{\mathrm{c}}\right)=\infty .
$$

Now $\phi(p)=0$ when $p>p_{\mathrm{c}}$ ( since $P_{p}\left(A_{n}\right) \geq \theta(p)>0$ ). Therefore the above discussion needs more thought in this case. In defining the supercritical correlation length, it is normal to work with the 'truncated' probabilities $P_{p}\left(A_{n},|C|<\infty\right)$. It may be shown $([95,165])$ that the limit

(6.8)

$$
\phi(p)=\lim _{n \rightarrow \infty}\left\{-\frac{1}{n} \log P_{p}(0 \leftrightarrow \partial B(n),|C|<\infty)\right\}
$$


exists for all $p$, and satisfies $\phi(p)>0$ if and only if $p \neq p_{\mathrm{c}}$. We now define the correlation length $\xi(p)$ by

$$
\xi(p)=1 / \phi(p) \quad \text { for } 0<p<1 .
$$

Proof of Theorem 6.2. Define the (two-point) connectivity function $\tau_{p}(x, y)=$ $P_{p}(x \leftrightarrow y)$. Using the FKG inequality,

$$
\tau_{p}(x, y) \geq P_{p}(\{x \leftrightarrow z\} \cap\{z \leftrightarrow y\}) \geq \tau_{p}(x, z) \tau_{p}(z, y)
$$

for any $z \in \mathbb{Z}^{d}$. Set $x=0, z=m e_{1}, y=(m+n) e_{1}$, to obtain that $\tau_{p}(r)=$ $P_{p}\left(0 \leftrightarrow r e_{1}\right)$ satisfies $\tau_{p}(m+n) \geq \tau_{p}(m) \tau_{p}(n)$. Therefore the limit $\phi_{2}(p)$ exists by the subadditive inequality.

The existence of $\phi_{1}(p)$ may be shown similarly, using the BK inequality as follows. Note that

$$
\{0 \leftrightarrow \partial B(m+n)\} \subseteq \bigcup_{x \in \partial B(m)}\{\{0 \leftrightarrow x\} \circ\{x \leftrightarrow x+\partial B(n)\}\}
$$

(this is geometry). Therefore $\beta_{p}(r)=P_{p}(0 \leftrightarrow \partial B(r))$ satisfies

$$
\beta_{p}(m+n) \leq \sum_{x \in \partial B(m)} \tau_{p}(0, x) \beta_{p}(n) .
$$

Now $\tau_{p}(0, x) \leq \beta_{p}(m)$ for $x \in \partial B(m)$, so that

$$
\beta_{p}(m+n) \leq|\partial B(m)| \beta_{p}(m) \beta_{p}(n) .
$$

With a little ingenuity, and the subadditive inequality, we deduce the existence of $\phi_{1}(p)$ in (6.3). That $\phi_{2}(p) \geq \phi_{1}(p)$ follows from the fact that $\tau_{p}\left(0, n e_{1}\right) \leq \beta_{p}(n)$. For the converse inequality, pick $x \in \partial B(n)$ such that

$$
\tau_{p}(0, x) \geq \frac{1}{|\partial B(n)|} \beta_{p}(n),
$$

and assume that $x_{1}=+n$. Now

$$
\tau_{p}\left(0,2 n e_{1}\right) \geq P_{p}\left(\{0 \leftrightarrow x\} \cap\left\{x \leftrightarrow 2 n e_{1}\right\}\right) \geq \tau_{p}(0, x)^{2}
$$

by the FKG inequality.
6.2 Exponential Decay

The target of this section is to prove exponential decay for connectivity functions when $p<p_{c}$, i.e., that the common limit $\phi(p)$ in $(6.3)-(6.4)$ is strictly positive when $0<p<p_{\mathrm{c}}$

Theorem 6.10. There exists $\psi(p)$, satisfying $\psi(p)>0$ when $0<p<p_{\mathrm{c}}$, such that

$$
P_{p}(0 \leftrightarrow \partial B(n)) \leq e^{-n \psi(p)} \quad \text { for all } n .
$$

It is straightforward to obtain inequality (6.11) with some $\psi(p)$ which is strictly positive when $p<(2 d-1)^{-1}$; just follow the proof of (3.4). The problem is to extend the conclusion from 'small positive $p$ ' to 'all subcritical values of $p$ '. Such a difficulty is canonical: one may often obtain estimates valid for sufficiently small (resp. large) $p$, but one may require such estimates all the way up to (resp. down to) the critical value $p_{\mathrm{c}}$.

We prove Theorem 6.10 via Menshikov's method $[268,271]$ rather than that of Aizenman-Barsky [13]. The proof given below is essentially a reproduction of that given in $[\mathrm{G}]$, but with the correction of a minor error on page 50 of $[\mathrm{G}]$. The equation, theorem, and figure numbers are taken unchanged from [G], pages $47-56^{1}$ It is a minor convenience here to work with the ball $S(n)=\left\{x \in \mathbb{Z}^{d}: \delta(0, x) \leq n\right\}$ containing all points within graph-theoretic distance $n$ of the origin. Note that $S(n)$ is a 'diamond' (see the forthcoming figure labelled Fig. 3.1), and write $A_{n}=$ $\{0 \leftrightarrow \partial S(n)\}$.

(The remainder of this section is extracted largely from [G])

Let $S(n, x)$ be the ball of radius $n$ with centre at the vertex $x$, and let $\partial S(n, x)$ be the surface of $S(n, x)$; thus $S(n, x)=x+S(n)$ and $\partial S(n, x)=x+\partial S(n)$. Similarly, let $A_{n}(x)$ be the event that there is an open path from the vertex $x$ to some vertex in $\partial S(n, x)$. We are concerned with the probabilities

$$
g_{p}(n)=P_{p}\left(A_{n}\right)=P_{p}\left(A_{n}(x)\right) \quad \text { for any } x .
$$

Now $A_{n}$ is an increasing event which depends on the edges joining vertices in $S(n)$ only. We apply Russo's formula to $P_{p}\left(A_{n}\right)$ to obtain

$$
g_{p}^{\prime}(n)=E_{p}\left(N\left(A_{n}\right)\right)
$$

where the prime denotes differentiation with respect to $p$, and $N\left(A_{n}\right)$ is the number of edges which are pivotal for $A_{n}$. It follows as in $(2.29)^{2}$ that

$$
\begin{aligned}
g_{p}^{\prime}(n) & =\frac{1}{p} E_{p}\left(N\left(A_{n}\right) ; A_{n}\right) \\
& =\frac{1}{p} E_{p}\left(N\left(A_{n}\right) \mid A_{n}\right) g_{p}(n)
\end{aligned}
$$

${ }^{1}$ Reproduced with the kind permission of Springer Verlag, which holds the copyright. ${ }^{2}$ See Theorem 42 of the current lecture notes. 


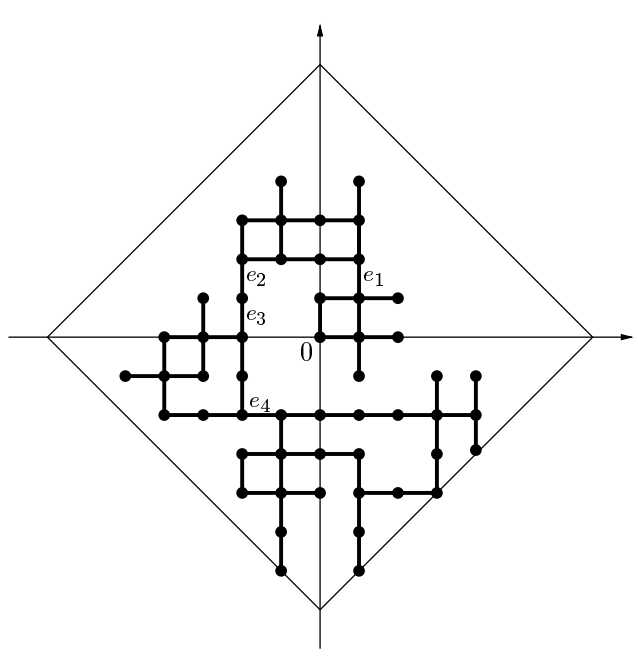

Fig. 3.1. A picture of the open cluster of $S(7)$ at the origin. There are exactly four pivotal edges for $A_{n}$ in this configuration, and these are labelled $e_{1}, e_{2}, e_{3}, e_{4}$

so that

$$
\frac{1}{g_{p}(n)} g_{p}^{\prime}(n)=\frac{1}{p} E_{p}\left(N\left(A_{n}\right) \mid A_{n}\right) .
$$

Let $0 \leq \alpha<\beta \leq 1$, and integrate (3.10) from $p=\alpha$ to $p=\beta$ to obtain

$$
\begin{aligned}
g_{\alpha}(n) & =g_{\beta}(n) \exp \left(-\int_{\alpha}^{\beta} \frac{1}{p} E_{p}\left(N\left(A_{n}\right) \mid A_{n}\right) d p\right) \\
& \leq g_{\beta}(n) \exp \left(-\int_{\alpha}^{\beta} E_{p}\left(N\left(A_{n}\right) \mid A_{n}\right) d p\right),
\end{aligned}
$$

as in (2.30). We need now to show that $E_{p}\left(N\left(A_{n}\right) \mid A_{n}\right)$ grows roughly linearly in $n$ when $p<p_{\mathrm{c}}$, and then this inequality will yield an upper bound for $g_{\alpha}(n)$ of the form required in (3.5). The vast majority of the work in the proof is devoted to estimating $E_{p}\left(N\left(A_{n}\right) \mid A_{n}\right)$, and the argument is roughly as follows. If $p<p_{\mathrm{c}}$ then $P_{p}\left(A_{n}\right) \rightarrow 0$ as $n \rightarrow \infty$, so that for large $n$ we are conditioning on an event of small probability. If $A_{n}$ occurs, 'but only just', then the connections between the origin and $\partial S(n)$ must be sparse; indeed, there must exist many open edges in $S(n)$ which are crucial for the occurrence of $A_{n}$ (see Figure 3.1). It is plausible that the number of such pivotal edges in paths from the origin to $\partial S(2 n)$ is approximately twice the number of such edges in paths to $\partial S(n)$, since these sparse paths have to traverse twice the distance. Thus the number $N\left(A_{n}\right)$ of edges pivotal for $A_{n}$ should grow linearly in $n$.
Suppose that the event $A_{n}$ occurs, and denote by $e_{1}, e_{2}, \ldots, e_{N}$ the (random) edges which are pivotal for $A_{n}$. Since $A_{n}$ is increasing, each $e_{j}$ has the property that $A_{n}$ occurs if and only if $e_{j}$ is open; thus all open paths from the origin to $\partial S(n)$ traverse $e_{j}$, for every $j$ (see Figure 3.1). Let $\pi$ be such an open path; we assume that the edges $e_{1}, e_{2} \ldots e_{N}$ have been enumerated in the order in which they are that the edges $e_{1}, e_{2}, \ldots, e_{N}$ have been enumerated in the order in which they are
traversed by $\pi$. A glance at Figure 3.1 confirms that this ordering is independent of the choice of $\pi$. We denote by $x_{i}$ the endvertex of $e_{i}$ encountered first by $\pi$, and by $y_{i}$ the other endvertex of $e_{i}$. We observe that there exist at least two edgedisjoint open paths joining 0 to $x_{1}$, since, if two such paths cannot be found then, by Menger's theorem (Wilson $1979^{3}$, p. 126), there exists a pivotal edge in $\pi$ which is encountered prior to $x_{1}$, a contradiction. Similarly, for $1 \leq i<N$, there exist at least two edge-disjoint open paths joining $y_{i}$ to $x_{i+1}$ : see Figure 3.2. In the words of the discoverer of this proof, the open cluster containing the origin resembles a chain of sausages.

As before, let $M=\max \left\{k: A_{k}\right.$ occurs $\}$ be the radius of the largest ball whose surface contains a vertex which is joined to the origin by an open path. We note that, if $p<p_{\mathrm{c}}$, then $M$ has a non-defective distribution in that $P_{p}(M \geq k)=g_{p}(k) \rightarrow 0$ as $k \rightarrow \infty$. We shall show that, conditional on $A_{n}, N\left(A_{n}\right)$ is at least as large as the number of renewals up to time $n$ of a renewal process whose inter-renewal times have approximately the same distribution as $M$. In order to compare $N\left(A_{n}\right)$ with such a renewal process, we introduce the following notation. Let $\rho_{1}=\delta\left(0, x_{1}\right)$ and $\rho_{i+1}=\delta\left(y_{i}, x_{i+1}\right)$ for $1 \leq i<N$. The first step is to show that, roughly speaking, the random variables $\rho_{1}, \rho_{2}, \ldots$ are jointly smaller in distribution than a sequence $M_{1}, M_{2}, \ldots$ of independent random variables distributed as $M$.

(3.12) Lemma. Let $k$ be a positive integer, and let $r_{1}, r_{2}, \ldots, r_{k}$ be non-negative integers such that $\sum_{i=1}^{k} r_{i} \leq n-k$. Then, for $0<p<1$,

(3.13) $P_{p}\left(\rho_{k} \leq r_{k}, \rho_{i}=r_{i}\right.$ for $\left.1 \leq i<k \mid A_{n}\right)$

$$
\geq P_{p}\left(M \leq r_{k}\right) P_{p}\left(\rho_{i}=r_{i} \text { for } 1 \leq i<k \mid A_{n}\right) .
$$

Proof. Suppose by way of illustration that $k=1$ and $0 \leq r_{1}<n$. Then

$$
\left\{\rho_{1}>r_{1}\right\} \cap A_{n} \subseteq A_{r_{1}+1} \circ A_{n},
$$

since if $\rho_{1}>r_{1}$ then the first endvertex of the first pivotal edge lies either outside $S\left(r_{1}+1\right)$ or on its surface $\partial S\left(r_{1}+1\right)$; see Figure 3.2. However, $A_{r_{1}+1}$ and $A_{n}$ are increasing events which depend on the edges within $S(n)$ only, and the BK inequality yields

$$
P_{p}\left(\left\{\rho_{1}>r_{1}\right\} \cap A_{n}\right) \leq P_{p}\left(A_{r_{1}+1}\right) P_{p}\left(A_{n}\right) .
$$

We divide by $P_{p}\left(A_{n}\right)$ to obtain

$$
P_{p}\left(\rho_{1}>r_{1} \mid A_{n}\right) \leq g_{p}\left(r_{1}+1\right) ;
$$

however $P_{p}(M \geq m)=g_{p}(m)$, and thus we have obtained (3.13) in the case $k=1$. 


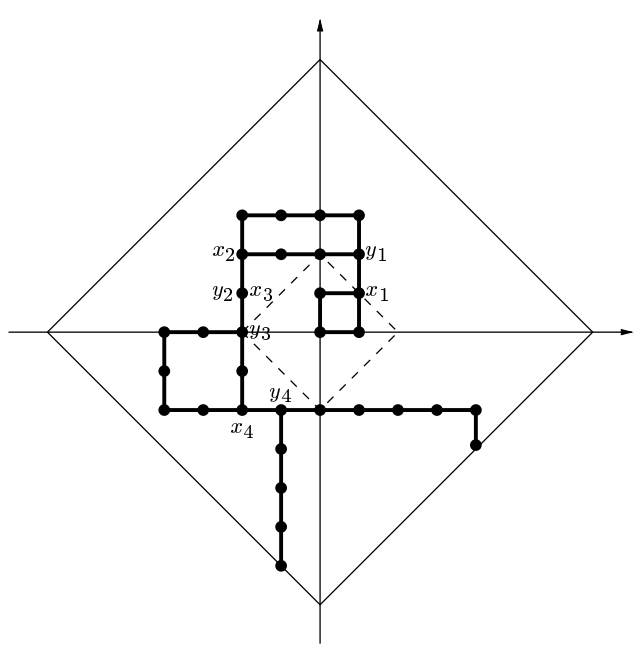

Fig. 3.2. The pivotal edges are $e_{i}=\left\langle x_{i}, y_{i}\right\rangle$ for $i=1,2,3,4$. Note that $x_{3}=y_{2}$ in this conf edge-disjoint paths from the origin to $\partial S\left(\rho_{1}\right)$

We now prove the lemma for general values of $k$. Suppose that $k \geq 1$, and let $r_{1}, r_{2} \ldots, r_{k}$ be non-negative integers with sum not exceeding $n-k$. Let $N$ be the number of edges which are pivotal for $A_{n}$; we enumerate and label these edges as $e_{i}=\left\langle x_{i}, y_{i}\right\rangle$ as before.

(The following section in italics replaces an incorrect passage in $[\mathrm{G}]$.)

For any edge $e=\langle u, v\rangle$, let $D_{e}$ be the set of vertices attainable from 0 along open paths not using e, together with all open edges between such vertices. Let $B_{e}$ be the event that the following statements hold:

(a) exactly one of $u$ or $v$ lies in $D_{e}$, say $u$,

(b) $e$ is open,

(c) De contains no vertex of $\partial S(n)$

(d) the pivotal edges for the event $\{0 \leftrightarrow v\}$ are, taken in order, $\left\langle x_{1}, y_{1}\right\rangle,\left\langle x_{2}, y_{2}\right\rangle$, $\ldots,\left\langle x_{k-2}, y_{k-2}\right\rangle,\left\langle x_{k-1}, y_{k-1}\right\rangle=e$, where $\delta\left(y_{i-1}, x_{i}\right)=r_{i}$ for $1 \leq i<k$, and $y_{0}=0$

We now define the event $B=\bigcup_{e} B_{e}$. For $\omega \in A_{n} \cap B$, there is a unique $e=e(\omega)$ such that $B_{e}$ occurs

For $\omega \in B$, we consider the set of vertices and open edges attainable along open paths from the origin without using $e=e(\omega)$; to this graph we append $e$ and its other endvertex $v=y_{k-1}$, and we place a mark over $y_{k-1}$ in order to distinguish it from the other vertices. We denote by $G=D_{e}$ the resulting (marked) graph, and we write $y(G)$ for the unique marked vertex of $G$. We condition on $G$ to obtain

$$
P_{p}\left(A_{n} \cap B\right)=\sum_{\Gamma} P_{p}(B, G=\Gamma) P_{p}\left(A_{n} \mid B, G=\Gamma\right),
$$

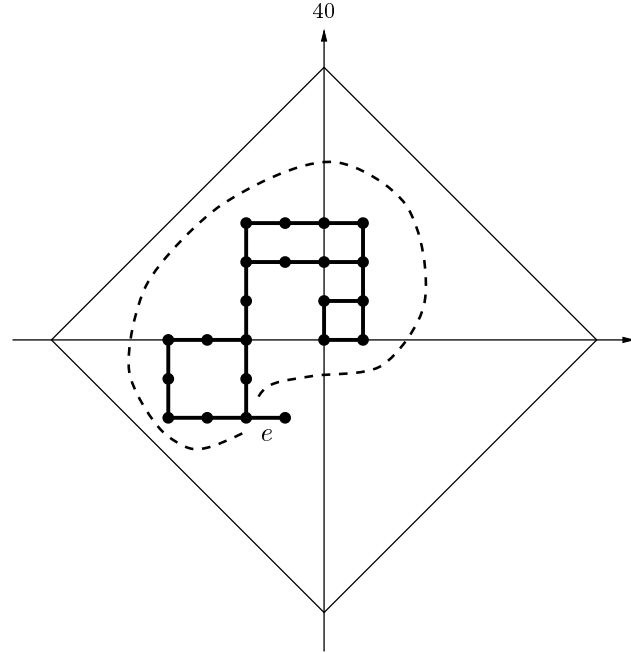

Fig. 3.3. A sketch of the event $B_{e}$. The dashed line indicates that the only open 'exit' from the interior is via the edge $e$. Note the existence of 3 pivotal edges for the event that 0 is connected to an endvertex of $e$

where the sum is over all possible values $\Gamma$ of $G$. The final term in this summation is the probability that $y(\Gamma)$ is joined to $\partial S(n)$ by an open path which has no vertex other than $y(\Gamma)$ in common with $\Gamma$. Thus, in the obvious terminology,

$$
P_{p}\left(A_{n} \cap B\right)=\sum_{\Gamma} P_{p}(B, G=\Gamma) P_{p}(y(\Gamma) \leftrightarrow \partial S(n) \text { off } \Gamma) .
$$

\section{Similarly,}

$$
\begin{aligned}
P_{p}\left(\left\{\rho_{k}>r_{k}\right\} \cap A_{n} \cap B\right) & =\sum_{\Gamma} P_{p}(B, G=\Gamma) P_{p}\left(\left\{\rho_{k}>r_{k}\right\} \cap A_{n} \mid B, G=\Gamma\right) \\
=\sum_{\Gamma} & P_{p}(B, G=\Gamma) \\
& \quad \times P_{p}\left(\left\{y(\Gamma) \leftrightarrow \partial S\left(r_{k}+1, y(\Gamma)\right) \text { off } \Gamma\right\} \circ\{y(\Gamma) \leftrightarrow \partial S(n) \text { off } \Gamma\}\right) .
\end{aligned}
$$

We apply the BK inequality to the last term to obtain

$(3.16$

$$
\begin{aligned}
& P_{p}\left(\left\{\rho_{k}>r_{k}\right\} \cap A_{n} \cap B\right) \\
& \quad \leq \sum_{\Gamma} P_{p}(B, G=\Gamma) P_{p}(y(\Gamma) \leftrightarrow \partial S(n) \text { off } \Gamma) P_{p}\left(y(\Gamma) \leftrightarrow \partial S\left(r_{k}+1, y(\Gamma)\right) \text { off } \Gamma\right) \\
& \quad \leq g_{p}\left(r_{k}+1\right) P_{p}\left(A_{n} \cap B\right)
\end{aligned}
$$


by (3.15) and the fact that, for each possible $\Gamma$,

$$
\begin{aligned}
P_{p}\left(y(\Gamma) \leftrightarrow \partial S\left(r_{k}+1, y(\Gamma)\right) \text { off } \Gamma\right) & \leq P_{p}\left(y(\Gamma) \leftrightarrow \partial S\left(r_{k}+1, y(\Gamma)\right)\right) \\
& =P_{p}\left(A_{r_{k}+1}\right) \\
& =g_{p}\left(r_{k}+1\right) .
\end{aligned}
$$

We divide each side of (3.16) by $P_{p}\left(A_{n} \cap B\right)$ to obtain

$$
P_{p}\left(\rho_{k} \leq r_{k} \mid A_{n} \cap B\right) \geq 1-g_{p}\left(r_{k}+1\right),
$$

throughout which we multiply by $P_{p}\left(B \mid A_{n}\right)$ to obtain the result.

(3.17) Lemma. For $0<p<1$, it is the case that

$$
E_{p}\left(N\left(A_{n}\right) \mid A_{n}\right) \geq \frac{n}{\sum_{i=0}^{n} g_{p}(i)}-1 .
$$

Proof. It follows from Lemma (3.12) that

(3.19) $\quad P_{p}\left(\rho_{1}+\rho_{2}+\cdots+\rho_{k} \leq n-k \mid A_{n}\right) \geq P\left(M_{1}+M_{2}+\cdots+M_{k} \leq n-k\right)$,

where $k \geq 1$ and $M_{1}, M_{2}, \ldots$ is a sequence of independent random variables distributed as $M$. We defer until the end of this proof the minor chore of deducing (3.19) from (3.13). Now $N\left(A_{n}\right) \geq k$ if $\rho_{1}+\rho_{2}+\cdots+\rho_{k} \leq n-k$, so that

$$
P_{p}\left(N\left(A_{n}\right) \geq k \mid A_{n}\right) \geq P\left(M_{1}+M_{2}+\cdots+M_{k} \leq n-k\right) .
$$

A minor difficulty is that the $M_{i}$ may have a defective distribution. Indeed,

$$
\begin{aligned}
P(M \geq r) & =g_{p}(r) \\
& \rightarrow \theta(p) \quad \text { as } r \rightarrow \infty ;
\end{aligned}
$$

thus we allow the $M_{i}$ to take the value $\infty$ with probability $\theta(p)$. On the other hand, we are not concerned with atoms at $\infty$, since

$$
P\left(M_{1}+M_{2}+\cdots+M_{k} \leq n-k\right)=P\left(M_{1}^{\prime}+M_{2}^{\prime}+\cdots+M_{k}^{\prime} \leq n\right),
$$

where $M_{i}^{\prime}=1+\min \left\{M_{i}, n\right\}$, and we work henceforth with these truncated random variables. Summing (3.20) over $k$, we obtain

$$
\begin{aligned}
E_{p}\left(N\left(A_{n}\right) \mid A_{n}\right) & \geq \sum_{k=1}^{\infty} P\left(M_{1}^{\prime}+M_{2}^{\prime}+\cdots+M_{k}^{\prime} \leq n\right) \\
& =\sum_{k=1}^{\infty} P(K \geq k+1) \\
& =E(K)-1,
\end{aligned}
$$

where $K=\min \left\{k: M_{1}^{\prime}+M_{2}^{\prime}+\cdots+M_{k}^{\prime}>n\right\}$. Let $S_{k}=M_{1}^{\prime}+M_{2}^{\prime}+\cdots+M_{k}^{\prime}$, the sum of independent, identically distributed, bounded random variables. By Wald's equation (see Chow and Teicher $1978^{4}$.pp. 137, 150),

$$
n<E\left(S_{K}\right)=E(K) E\left(M_{1}^{\prime}\right),
$$

giving that

$$
E(K)>\frac{n}{E\left(M_{1}^{\prime}\right)}=\frac{n}{1+E\left(\min \left\{M_{1}, n\right\}\right)}=\frac{n}{\sum_{i=0}^{n} g_{p}(i)}
$$

since

$$
E\left(\min \left\{M_{1}, n\right\}\right)=\sum_{i=1}^{n} P(M \geq i)=\sum_{i=1}^{n} g_{p}(i)
$$

It remains to show that (3.19) follows from Lemma (3.12). We have that

$$
\begin{aligned}
P_{p}\left(\rho_{1}\right. & \left.+\rho_{2}+\cdots+\rho_{k} \leq n-k \mid A_{n}\right) \\
& =\sum_{i=0}^{n-k} P_{p}\left(\rho_{1}+\rho_{2}+\cdots+\rho_{k-1}=i, \rho_{k} \leq n-k-i \mid A_{n}\right) \\
& \geq \sum_{i=0}^{n-k} P(M \leq n-k-i) P_{p}\left(\rho_{1}+\rho_{2}+\cdots+\rho_{k-1}=i \mid A_{n}\right) \quad \text { by }(3.13) \\
& =P_{p}\left(\rho_{1}+\rho_{2}+\cdots+\rho_{k-1}+M_{k} \leq n-k \mid A_{n}\right),
\end{aligned}
$$

where $M_{k}$ is a random variable which is independent of all edge-states in $S(n)$ and is distributed as $M$. There is a mild abuse of notation here, since $P_{p}$ is not the correct probability measure unless $M_{k}$ is measurable on the usual $\sigma$-field of events, but we need not trouble ourselves overmuch about this. We iterate the above argument in the obvious way to deduce (3.19), thereby completing the proof of the lemma.

The conclusion of Theorem (3.8) is easily obtained from this lemma, but we delay this step until the end of the section. The proof of Theorem (3.4) proceeds by substituting (3.18) into (3.11) to obtain that, for $0 \leq \alpha<\beta \leq 1$,

$$
g_{\alpha}(n) \leq g_{\beta}(n) \exp \left(-\int_{\alpha}^{\beta}\left[\frac{n}{\sum_{i=0}^{n} g_{p}(i)}-1\right] d p\right) .
$$

It is difficult to calculate the integral in the exponent, and so we use the inequality $g_{p}(i) \leq g_{\beta}(i)$ for $p \leq \beta$ to obtain

(3.22) $\quad g_{\alpha}(n) \leq g_{\beta}(n) \exp \left(-(\beta-\alpha)\left[\frac{n}{\sum_{i=0}^{n} g_{\beta}(i)}-1\right]\right)$,

${ }^{4}$ Reference [107]. 
and it is from this relation that the conclusion of Theorem (3.4) will be extracted. Before continuing, it is interesting to observe that by combining (3.10) and (3.18) we obtain a differential-difference inequality involving the function

$$
G(p, n)=\sum_{i=0}^{n} g_{p}(i)
$$

rewriting this equation rather informally as a partial differential inequality, we obtain

$$
\frac{\partial^{2} G}{\partial p \partial n} \geq \frac{\partial G}{\partial n}\left(\frac{n}{G}-1\right)
$$

Efforts to integrate this inequality directly have failed so far.

Once we know that

$$
E_{\beta}(M)=\sum_{i=1}^{\infty} g_{\beta}(i)<\infty \quad \text { for all } \beta<p_{\mathrm{c}},
$$

then $(3.22)$ gives us that

$$
g_{\alpha}(n) \leq e^{-n \psi(\alpha)} \quad \text { for all } \alpha<p_{\mathrm{c}}
$$

for some $\psi(\alpha)>0$, as required. At the moment we know rather less than the finite summability of the $g_{p}(i)$ for $p<p_{\mathrm{c}}$, knowing only that $g_{p}(i) \rightarrow 0$ as $i \rightarrow \infty$. In order to estimate the rate at which $g_{p}(i) \rightarrow 0$, we shall use $(3.22)$ as a mathematical turbocharger.

(3.24) Lemma. For $p<p_{\mathrm{c}}$, there exists $\delta(p)$ such that

$$
g_{p}(n) \leq \delta(p) n^{-1 / 2} \quad \text { for } n \geq 1 .
$$

Once this lemma has been proved, the theorem follows quickly. To see this, note that (3.25) implies the existence of $\Delta(p)<\infty$ such that

$$
\sum_{i=0}^{n} g_{p}(i) \leq \Delta(p) n^{1 / 2} \quad \text { for } p<p_{\mathrm{c}} .
$$

Let $\alpha<p_{\mathrm{c}}$, and find $\beta$ such that $\alpha<\beta<p_{\mathrm{c}}$. Substitute (3.26) with $p=\beta$ into (3.22) to find that

$$
\begin{aligned}
g_{\alpha}(n) & \leq g_{\beta}(n) \exp \left\{-(\beta-\alpha)\left(\frac{n^{1 / 2}}{\Delta(\beta)}-1\right)\right\} \\
& \leq \exp \left\{1-\frac{(\beta-\alpha)}{\Delta(\beta)} n^{1 / 2}\right\}
\end{aligned}
$$

Thus

$$
\sum_{n=1}^{\infty} g_{\alpha}(n)<\infty \quad \text { for } \alpha<p_{\mathrm{c}}
$$

and the theorem follows from the observations made prior to the statement of Lemma (3.24). We shall now prove this lemma.

Proof. First, we shall show the existence of a subsequence $n_{1}, n_{2}, \ldots$ along which $g_{p}(n)$ approaches 0 rather quickly; secondly, we shall fill in the gaps in this subsequence.

Fix $\beta<p_{c}$ and a positive integer $n$. Let $\alpha$ satisfy $0<\alpha<\beta$ and let $n^{\prime} \geq n$ later we shall choose $\alpha$ and $n^{\prime}$ explicitly in terms of $\beta$ and $n$. From (3.22),

$$
\begin{aligned}
g_{\alpha}\left(n^{\prime}\right) & \leq g_{\beta}\left(n^{\prime}\right) \exp \left(1-\frac{n^{\prime}(\beta-\alpha)}{\sum_{i=0}^{n^{\prime}} g_{\beta}(i)}\right) \\
& \leq g_{\beta}(n) \exp \left(1-\frac{n^{\prime}(\beta-\alpha)}{\sum_{i=0}^{n^{\prime}} g_{\beta}(i)}\right)
\end{aligned}
$$

since $n \leq n^{\prime}$. We wish to write the exponent in terms of $g_{\beta}(n)$, and to this end we shall choose $n^{\prime}$ appropriately. We split the summation into two parts corresponding to $i<n$ and $i \geq n$, and we use the monotonicity of $g_{\beta}(i)$ to find that

$$
\begin{aligned}
\frac{1}{n^{\prime}} \sum_{i=0}^{n^{\prime}} g_{\beta}(i) & \leq \frac{1}{n^{\prime}}\left\{n g_{\beta}(0)+n^{\prime} g_{\beta}(n)\right\} \\
& \leq 3 g_{\beta}(n) \quad \text { if } n^{\prime} \geq n\left\lfloor g_{\beta}(n)^{-1}\right\rfloor .
\end{aligned}
$$

We now define

$$
n^{\prime}=n \gamma_{\beta}(n) \quad \text { where } \gamma_{\beta}(n)=\left\lfloor g_{\beta}(n)^{-1}\right\rfloor
$$

and deduce from (3.27) that

$$
g_{\alpha}\left(n^{\prime}\right) \leq g_{\beta}(n) \exp \left(1-\frac{\beta-\alpha}{3 g_{\beta}(n)}\right)
$$

Next we choose $\alpha$ by setting

$$
\beta-\alpha=3 g_{\beta}(n)\left\{1-\log g_{\beta}(n)\right\} .
$$

Now $g_{\beta}(m) \rightarrow 0$ as $m \rightarrow \infty$, so that $0<\alpha<\beta$ if $n$ has been picked large enough; (3.29) then yields

$$
g_{\alpha}\left(n^{\prime}\right) \leq g_{\beta}(n)^{2} .
$$

This conclusion is the basic recursion step which we shall use repeatedly. We have shown that, for $\beta<p_{\mathrm{c}}$, there exists $n_{0}(\beta)$ such that (3.31) holds for all $n \geq n_{0}(\beta)$ whenever $n^{\prime}$ and $\alpha$ are given by (3.28) and (3.30), respectively.

Next, we fix $p<p_{\mathrm{c}}$ and choose $\pi$ such that $p<\pi<p_{\mathrm{c}}$. We now construct sequences $\left(p_{i}: i \geq 0\right)$ of probabilities and $\left(n_{i}: i \geq 0\right)$ of integers as follows. We set 

$p_{0}=\pi$ and shall pick $n_{0}$ later. Having found $p_{0}, p_{1}, \ldots, p_{i}$ and $n_{0}, n_{1}, \ldots, n_{i}$, we
define

$(3.32$

$$
n_{i+1}=n_{i} \gamma_{i} \quad \text { and } \quad p_{i}-p_{i+1}=3 g_{i}\left(1-\log g_{i}\right)
$$

where $g_{i}=g_{p_{i}}\left(n_{i}\right)$ and $\gamma_{i}=\left\lfloor g_{i}^{-1}\right\rfloor$. We note that $n_{i} \leq n_{i+1}$ and $p_{i}>p_{i+1}$. The recursion (3.32) is valid so long as $p_{i+1}>0$, and this is indeed the case so long as $n_{0}$ has been chosen to be sufficiently large. To see this we argue as follows. From the definition of $p_{0}, \ldots, p_{i}$ and $n_{0}, \ldots, n_{i}$ and the discussion leading to (3.31), we find that

\section{(3.33)}

$$
g_{j+1} \leq g_{j}^{2} \text { for } j=0,1, \ldots, i-1 .
$$

If a real sequence $\left(x_{j}: j \geq 0\right)$ satisfies $0<x_{0}<1, x_{j+1}=x_{j}^{2}$ for $j \geq 0$, then it is easy to check that

$$
s\left(x_{0}\right)=\sum_{j=0}^{\infty} 3 x_{j}\left(1-\log x_{j}\right)<\infty,
$$

and furthermore that $s\left(x_{0}\right) \rightarrow 0$ as $x_{0} \rightarrow 0$. We may pick $x_{0}$ sufficiently small such that

\section{$(3.34)$}

$$
s\left(x_{0}\right) \leq \pi-p
$$

and then we pick $n_{0}$ sufficiently large that $g_{0}=g_{\pi}\left(n_{0}\right)<x_{0}$. Now $h(x)=$ $3 x(1-\log x)$ is an increasing function on $\left[0, x_{0}\right]$, giving from (3.32) and (3.33) that

$$
\begin{aligned}
p_{i+1} & =p_{i}-3 g_{i}\left(1-\log g_{i}\right) \\
& =\pi-\sum_{j=0}^{i} 2 g_{j}\left(1-\log g_{j}\right) \\
& \geq \pi-\sum_{j=0}^{\infty} 3 x_{j}\left(1-\log x_{j}\right) \\
& \geq p \quad \text { by }(3.34) .
\end{aligned}
$$

Thus, by a suitable choice of $n_{0}$ we may guarantee not only that $p_{i+1}>0$ for all $i$ but also that

$$
\widetilde{p}=\lim _{i \rightarrow \infty} p_{i}
$$

satisfies $\widetilde{p} \geq p$. Let us suppose that $n_{0}$ has been chosen accordingly, so that the recursion (3.32) is valid and $\widetilde{p} \geq p$. We have from (3.32) and (3.33) that

$$
n_{k}=n_{0} \gamma_{0} \gamma_{1} \ldots \gamma_{k-1} \quad \text { for } k \geq 1
$$

and

$$
(3.35)
$$

$$
\begin{aligned}
g_{k-1}^{2} & =g_{k-1} g_{k-1} \\
& \leq g_{k-1} g_{k-2}^{2} \leq \cdots \\
& \leq g_{k-1} g_{k-2} \ldots g_{1} g_{0}^{2} \\
& \leq\left(\gamma_{k-1} \gamma_{k-2} \ldots \gamma_{0}\right)^{-1} g_{0} \\
& =\delta^{2} n_{k}^{-1}
\end{aligned}
$$

where $\delta^{2}=n_{0} g_{0}$.

We are essentially finished. Let $n>n_{0}$, and find an integer $k$ such that $n_{k-1} \leq$ $n<n_{k}$; this is always possible since $g_{k} \rightarrow 0$ as $k \rightarrow \infty$, and therefore $n_{k-1}<n_{k}$ for all large $k$. Then

$$
\begin{aligned}
g_{p}(n) & \leq g_{p_{k-1}}\left(n_{k-1}\right) & & \text { since } p \leq p_{k-1} \\
& =g_{k-1} & & \\
& \leq \delta n_{k}^{-1 / 2} & & \text { by }(3.35) \\
& \leq \delta n^{-1 / 2} & & \text { since } n<n_{k}
\end{aligned}
$$

as required. This is valid for $n>n_{0}$, but we may adjust the constant $\delta$ so that a similar inequality is valid for all $n \geq 1$.

\subsection{ORNSTEIN-ZERNIKE DeCAY}

The connectivity function $\tau_{p}(x, y)=P_{p}(x \leftrightarrow y)$ decays exponentially when $p<p_{\mathrm{c}}$, which is to say that the limits

$$
\phi(p, x)=\lim _{n \rightarrow \infty}\left\{-\frac{1}{n} \log \tau_{p}(0, n x)\right\}
$$

exist and satisfy $\phi(p, x)>0$ for $0<p<p_{\mathrm{c}}$ and $x \in \mathbb{Z}^{d} \backslash\{0\}$ (cf. Theorem 6.2).

In one direction, this observation may lead to a study of the function $\phi(p, \cdot)$. In another, one may ask for finer asymptotics in (6.12). We concentrate on the case $x=e_{1}$, and write $\phi(p)=\phi\left(p, e_{1}\right)$

Theorem 6.13 (Ornstein-Zernike Decay). Suppose that $0<p<p_{\mathrm{c}}$. There exists a positive function $A(p)$ such that

$$
\tau_{p}\left(0, n e_{1}\right)=\left(1+\mathrm{O}\left(n^{-1}\right)\right) \frac{A(p)}{n^{\frac{1}{2}(d-1)}} e^{-n \phi(p)} \quad \text { as } n \rightarrow \infty .
$$

The correction factor $n^{-\frac{1}{2}(d-1)}$ occurs similarly in many other disordered systems, as was proposed by Ornstein and Zernike [301]. Theorem 6.13, and certain extensions, was obtained for percolation by Campanino, Chayes, and Chayes [86]. 


\section{SUPERCRITICAL PERCOLATION}

7.1 Uniqueness of the Infinite Cluster

Let $I$ be the number of infinite open clusters.

Theorem 7.1. For any $p$, either $P_{p}(I=0)=1$ or $P_{p}(I=1)=1$.

This result was proved first in [21], then more briefly in [146], and the definitive proof of Burton and Keane [83] appeared shortly afterwards. This last proof is short and elegant, and relies only on the zero-one law and a little geometry.

Proof. Fix $p \in[0,1]$. The sample space $\Omega=\{0,1\}^{\mathbb{E}}$ is a product space with a natural family of translations inherited from the translations of the lattice $\mathbb{L}^{d}$. Furthermore, $P_{p}$ is a product measure on $\Omega$. Since $I$ is a translation-invariant function on $\Omega$, it is a.s. constant, which is to say that

$$
\text { there exists } k \in\{0,1, \ldots\} \cup\{\infty\} \text { such that } P_{p}(I=k)=1 .
$$

Naturally, the value of $k$ depends on the choice of $p$. Next we show that the $k$ in question satisfies $k \in\{0,1, \infty\}$. Suppose (7.2) holds with some $k$ satisfying $2 \leq k<\infty$. We may find a box $B$ sufficiently large that

$$
P_{p}(B \text { intersects two or more infinite clusters })>\frac{1}{2} .
$$

By changing the states of edges in $B$ (by making all such edges open, say) we can decrease the number of infinite clusters (on the event in (7.3)). Therefore $P_{p}(I=k-1)>0$, in contradiction of $(7.2)$. Therefore we cannot have $2 \leq k<\infty$ in $(7.2)$.

It remains to rule out the case $k=\infty$. Suppose that $k=\infty$. We will derive a contradiction by using a geometrical argument. We call a vertex $x$ a trifurcation if:

(a) $x$ lies in an infinite open cluster, and

(b) the deletion of $x$ splits this infinite cluster into exactly three disjoint infinite clusters and no finite clusters,

and we denote by $T_{x}$ the event that $x$ is a trifurcation. Now $P_{p}\left(T_{x}\right)$ is constant for all $x$, and therefore

$$
\frac{1}{|B(n)|} E_{p}\left(\sum_{x \in B(n)} 1_{T_{x}}\right)=P_{p}\left(T_{0}\right)
$$

(Recall that $1_{A}$ denotes the indicator function of an event $A$.) It is useful to know that the quantity $P_{p}\left(T_{0}\right)$ is strictly positive, and it is here that we use the assumed multiplicity of infinite clusters. Since $P_{p}(I=\infty)=1$ by assumption, we may find a box $B(n)$ sufficiently large that it intersects at least three distinct infinite clusters with probability at least $\frac{1}{2}$. By changing the configuration inside $B(n)$, we

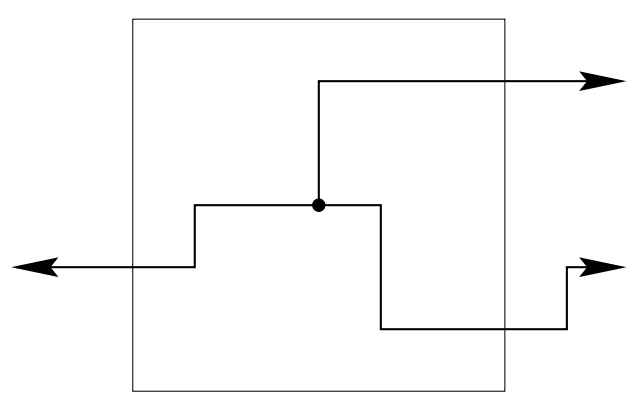

Fig. 7.1. Take a box $B$ which intersects at least three distinct infinite open clusters, and then alter the configuration inside $B$ in order to create a configuration in which 0 is a trifurcation

may turn the origin into a trifurcation (see Figure 7.1). The corresponding set of configurations has strictly positive probability, so that $P_{p}\left(T_{0}\right)>0$ in (7.4).

Before turning to the geometry, we present a lemma concerning partitions. Let $Y$ be a finite set with $|Y| \geq 3$, and define a 3-partition $\Pi=\left\{P_{1}, P_{2}, P_{3}\right\}$ of $Y$ to be a partition of $Y$ into exactly three non-empty sets $P_{1}, P_{2}, P_{3}$. For 3-partitions $\Pi=\left\{P_{1}, P_{2}, P_{3}\right\}$ and $\Pi^{\prime}=\left\{P_{1}^{\prime}, P_{2}^{\prime}, P_{3}^{\prime}\right\}$, we say that $\Pi$ and $\Pi^{\prime}$ are compatible if there exists an ordering of their elements such that $P_{1} \supseteq P_{2}^{\prime} \cup P_{3}^{\prime}$ (or, equivalently, that $\left.P_{1}^{\prime} \supseteq P_{2} \cup P_{3}\right)$. A collection $\mathcal{P}$ of 3-partitions is compatible if each pair therein is compatible.

Lemma 7.5. If $\mathcal{P}$ is a compatible family of distinct 3-partitions of $Y$, then $|\mathcal{P}| \leq$ $|Y|-2$.

Proof. There are several ways of doing this; see [83]. For any set $\mathcal{Q}$ of distinct compatible 3-partitions of $Y$, we define an equivalence relation $\sim$ on $Y$ by $x \sim y$ if, for all $\Pi \in \mathcal{Q}, x$ and $y$ lie in the same element of $\Pi$. Write $\alpha(\mathcal{Q})$ for the number of equivalence classes of $\sim$. Now, write $\mathcal{P}=\left(\Pi_{1}, \Pi_{2}, \ldots, \Pi_{m}\right)$ in some order, and let $\alpha_{k}=\alpha\left(\Pi_{1}, \Pi_{2}, \ldots, \Pi_{k}\right)$. Evidently $\alpha_{1}=3$ and, using the compatibility of $\Pi_{1}$ and $\Pi_{2}$, we have that $\alpha_{2} \geq 4$. By comparing $\Pi_{r+1}$ with $\Pi_{1}, \Pi_{2}, \ldots, \Pi_{r}$ in turn, and using their compatibility, one sees that $\alpha\left(\Pi_{1}, \Pi_{2}, \ldots, \Pi_{r+1}\right) \geq \alpha\left(\Pi_{1}, \Pi_{2}, \ldots, \Pi_{r}\right)+1$, whence $\alpha_{m} \geq \alpha_{1}+(m-1)=m+2$. However $\alpha_{m} \leq|Y|$, and the claim of the lemma follows.

Let $K$ be a connected open cluster of $B(n)$, and write $\partial K=K \cap \partial B(n)$. If $x(\in B(n-1))$ is a trifurcation in $K$, then the removal of $x$ induces a 3-partition $\Pi_{K}(x)=\left\{P_{1}, P_{2}, P_{3}\right\}$ of $\partial K$ with the properties that

(a) $P_{i}$ is non-empty, for $i=1,2,3$,

(b) $P_{i}$ is a subset of a connected subgraph of $B(n) \backslash\{x\}$,

(c) $P_{i} \leftrightarrow P_{j}$ in $B(n)$, if $i \neq j$.

Furthermore, if $x$ and $x^{\prime}$ are distinct trifurcations of $K \cap B(n-1)$, then $\Pi_{K}(x)$ and $\Pi_{K}\left(x^{\prime}\right)$ are distinct and compatible; see Figure 7.2. 


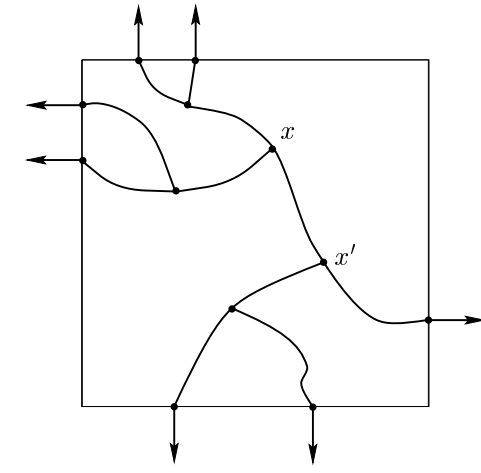

Fig. 7.2. Two trifurcations $x$ and $x^{\prime}$ belonging to a cluster $K$ of $B(n)$. They induce compatible partitions of $\partial K$.

It follows by Lemma 7.5 that the number $T(K)$ of trifurcations in $K \cap B(n-1)$ satisfies

$$
T(K) \leq|\partial K|-2 .
$$

We sum this inequality over all connected clusters of $B(n)$, to obtain that

$$
\sum_{x \in B(n-1)} 1_{T_{x}} \leq|\partial B(n)| .
$$

Take expectations, and use (7.4) to find that

$$
|B(n-1)| P_{p}\left(T_{0}\right) \leq|\partial B(n)|,
$$

which is impossible for large $n$ since the left side grows as $n^{d}$ and the right side as $n^{d-1}$. This contradiction completes the proof.

\subsection{Percolation in Slabs}

Many results were proved for subcritical percolation under the hypothesis of 'finite susceptibility', i.e., that $\chi(p)=E_{p}|C|$ satisfies $\chi(p)<\infty$. Subsequently, it was proved in $[13,268,271]$ that this hypothesis is satisfied whenever $p<p_{\mathrm{c}}$. The situation was similar for supercritical percolation, the corresponding hypothesis being that percolation occurs in slabs. We define the slab of thickness $k$ by

$$
S_{k}=\mathbb{Z}^{d-1} \times\{0,1, \ldots, k\},
$$

with critical probability $p_{\mathrm{c}}\left(S_{k}\right)$; we assume here that $d \geq 3$. The decreasing limit $p_{\mathrm{c}}(S)=\lim _{k \rightarrow \infty} p_{\mathrm{c}}\left(S_{k}\right)$ exists, and satisfies $p_{\mathrm{c}}(S) \geq p_{\mathrm{c}}$. The hypothesis of 'percolation in slabs' is that $p>p_{\mathrm{c}}(S)$. Here is an example of the hypothesis in action (cf. Theorem 6.2 and equation (6.8))
Theorem 7.6. The limit

$$
\sigma(p)=\lim _{n \rightarrow \infty}\left\{-\frac{1}{n} \log P_{p}(0 \leftrightarrow \partial B(n),|C|<\infty)\right\}
$$

exists. Furthermore $\sigma(p)>0$ if $p>p_{\mathrm{c}}(S)$.

This theorem asserts the exponential decay of a 'truncated' connectivity function when $d \geq 3$. Corresponding results when $d=2$ may be proved using duality.

Proof. The existence of the limit is an exercise in subadditivity (see [95, G]), and we sketch here only a proof that $\sigma(p)>0$. Assume that $p>p_{\mathrm{c}}(S)$, so that $p>p_{\mathrm{c}}\left(S_{k}\right)$ for some $k$; choose $k$ accordingly. Let $H_{n}$ be the hyperplane containing all vertices $x$ with $x_{1}=n$. It suffices to prove that

$$
P_{p}\left(0 \leftrightarrow H_{n},|C|<\infty\right) \leq e^{-\gamma n}
$$

for some $\gamma=\gamma(p)>0$. Define the slabs

$$
T_{i}=\left\{x \in \mathbb{Z}^{d}:(i-1) k \leq x_{1}<i k\right\}, \quad 1 \leq i<\lfloor n / k\rfloor .
$$

Any path from 0 to $H_{n}$ must traverse every such slab. Since $p>p_{\mathrm{c}}\left(S_{k}\right)$, each slab a.s. contains an infinite open cluster. If $0 \leftrightarrow H_{n}$ and $|C|<\infty$, then all paths from is paid for each. With a touch of rigour, this argument implies that

$$
P_{p}\left(0 \leftrightarrow H_{n},|C|<\infty\right) \leq\left\{1-\theta_{k}(p)\right\}^{\lfloor n / k\rfloor}
$$

where

$$
\theta_{k}(p)=P_{p}\left(0 \leftrightarrow \infty \text { in } S_{k}\right)>0 .
$$

For more details, see $[\mathrm{G}]$.

Grimmett and Marstrand [165] proved that $p_{\mathrm{c}}=p_{\mathrm{c}}(S)$, using ideas similar to those of $[49,50]$. This was achieved via a 'block construction' which appears to be central to a full understanding of supercritical percolation and to have further applications elsewhere. The details are presented next.

7.3 Limit of Slab Critical Points

Material in this section is taken from [165]. We assume that $d \geq 3$ and that $p$ is such that $\theta(p)>0$; under this hypothesis, we wish to gain some control of the (a.s.) unique open cluster. In particular we shall prove the following theorem, in which $p_{c}(A)$ denotes the critical value of bond percolation on the subgraph of $\mathbb{Z}^{d}$ induced by the vertex set $A$. In this notation, $p_{\mathrm{c}}=p_{\mathrm{c}}\left(\mathbb{Z}^{d}\right)$. 
Theorem 7.8. If $F$ is an infinite connected subset of $\mathbb{Z}^{d}$ with $p_{\mathrm{c}}(F)<1$, then for each $\eta>0$ there exists an integer $k$ such that

$$
p_{\mathrm{c}}(2 k F+B(k)) \leq p_{\mathrm{c}}+\eta .
$$

Choosing $F=\mathbb{Z}^{2} \times\{0\}^{d-2}$, we have that $2 k F+B(k)=\left\{x \in \mathbb{Z}^{d}:-k \leq x_{j} \leq\right.$ $k$ for $3 \leq j \leq d\}$. The theorem implies that $p_{\mathrm{c}}(2 k F+B(k)) \rightarrow p_{\mathrm{c}}$ as $k \rightarrow \infty$, which is a stronger statement than the statement that $p_{\mathrm{c}}=p_{\mathrm{c}}(S)$.

In the remainder of this section, we sketch the salient features of the block construction necessary to prove the above theorem. This construction may be used directly to obtain further information concerning supercritical percolation.

The main idea involves working with a 'block lattice' each point of which represents a large box of $\mathbb{L}^{d}$, these boxes being disjoint and adjacent. In this block lattice, we declare a vertex to be 'open' if there exist certain open paths in and near the corresponding box of $\mathbb{L}^{d}$. We shall show that, with positive probability, there exists an infinite path of open vertices in the block lattice. Furthermore, this infinite path of open blocks corresponds to an infinite open path of $\mathbb{L}^{d}$. By choosing sufficiently large boxes, we aim to find such a path within a sufficiently wide slab. Thus there is a probabilistic part of the proof, and a geometric part.

There are two main steps in the proof. In the first, we show the existence of long finite paths. In the second, we show how to take such finite paths and build an infinite cluster in a slab.

The principal parts of the first step are as follows. Pick $p$ such that $\theta(p)>0$

1. Let $\epsilon>0$. Since $\theta(p)>0$, there exists $m$ such that

$$
P_{p}(B(m) \leftrightarrow \infty)>1-\epsilon .
$$

This is elementary probability theory.

2. Let $n \geq 2 m$, say, and let $k \geq 1$. We may choose $n$ sufficiently large to ensure that, with probability at least $1-2 \epsilon, B(m)$ is joined to at least $k$ points in $\partial B(n)$.

3. By choosing $k$ sufficiently large, we may ensure that, with probability at least $1-3 \epsilon, B(m)$ is joined to some point of $\partial B(n)$, which is itself connected to a copy of $B(m)$, lying 'on' the surface $\partial B(n)$ and every edge of which is open. 4. The open copy of $B(m)$, constructed above, may be used as a 'seed' for iterating the above construction. When doing this, we shall need some contro over where the seed is placed. It may be shown that every face of $\partial B(n)$ contains (with large probability) a point adjacent to some seed, and indeed many such points.

Above is the scheme for constructing long finite paths, and we turn to the second step.

5. This construction is now iterated. At each stage there is a certain (small) probability of failure. In order that there be a strictly positive probability of an infinite sequence of successes, we iterate 'in two independent directions'. With care, one may show that the construction dominates a certain supercritical site percolation process on $\mathbb{L}^{2}$.

6. We wish to deduce that an infinite sequence of successes entails an infinite open path of $\mathbb{L}^{d}$ within the corresponding slab. There are two difficulties with this. First, since there is not total control of the positions of the seeds, the actual path in $\mathbb{L}^{d}$ may leave every slab. This may be overcome by a process of 'steering', in which, at each stage, we choose a seed in such a position as to compensate for any earlier deviation in space.

7. A larger problem is that, in iterating the construction, we carry with us a mixture of 'positive' and 'negative' information (of the form that 'certain paths exist' and 'others do not'). In combining events we cannot use the FKG inequality. The practical difficulty is that, although we may have an infinite sequence of successes, there will generally be breaks in any corresponding open route to $\infty$. This is overcome by sprinkling down a few more open edges, i.e., by working at edge-density $p+\delta$ where $\delta>0$, rather than at $p$.

In conclusion, we show that, if $\theta(p)>0$ and $\delta>0$, then there is (with large probability) an infinite $(p+\delta)$-open path in a slice of the form

$$
T_{k}=\left\{x \in \mathbb{Z}^{d}: 0 \leq x_{j} \leq k \text { for } j \geq 3\right\}
$$

where $k$ is sufficiently large. This implies that $p+\delta>p_{\mathrm{c}}(T)=\lim _{k \rightarrow \infty} p_{\mathrm{c}}\left(T_{k}\right)$ if $p>p_{\mathrm{c}}$, i.e., that $p_{\mathrm{c}} \geq p_{\mathrm{c}}(T)$. Since $p_{\mathrm{c}}(T) \geq p_{\mathrm{c}}$ by virtue of the fact that $T_{k} \subseteq \mathbb{Z}$ for all $k$, we may conclude that $p_{\mathrm{c}}=p_{\mathrm{c}}(T)$, implying also that $p_{\mathrm{c}}=p_{\mathrm{c}}(S)$.

Henceforth we suppose that $d=3$; similar arguments are valid when $d>3$. We begin with some notation and two key lemmas. As usual, $B(n)=[-n, n]^{3}$, and we shall concentrate on a special face of $B(n)$,

$$
F(n)=\left\{x \in \partial B(n): x_{1}=n\right\},
$$

and indeed on a special 'quadrant' of $F(n)$,

$$
T(n)=\left\{x \in \partial B(n): x_{1}=n, x_{j} \geq 0 \text { for } j \geq 2\right\} .
$$

For $m, n>1$, let

$$
T(m, n)=\bigcup_{j=1}^{2 m+1}\left\{j e_{1}+T(n)\right\}
$$

where $e_{1}=(1,0,0)$ as usual.

We call a box $x+B(m)$ a seed if every edge in $x+B(m)$ is open. We now set

$$
K(m, n)=\left\{x \in T(n):\left\langle x, x+e_{1}\right\rangle\right. \text { is open, and }
$$$$
\left.x+e_{1} \text { lies in some seed lying within } T(m, n)\right\} .
$$

The random set $K(m, n)$ is necessarily empty if $n<2 m$. 


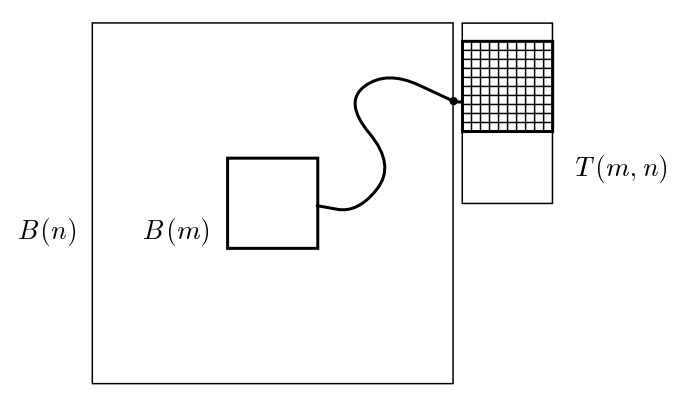

Fig. 7.3. An illustration of the event in (7.10). The hatched region is a copy of $B(m)$ all of whose edges are $p$-open. The central box $B(m)$ is joined by a path to $B(n)$.

Lemma 7.9. If $\theta(p)>0$ and $\eta>0$, there exists $m=m(p, \eta)$ and $n=n(p, \eta)$ such that $2 m<n$ and

$$
P_{p}(B(m) \leftrightarrow K(m, n) \text { in } B(n))>1-\eta .
$$

The event in (7.10) is illustrated in Figure 7.3.

Proof. Since $\theta(p)>0$, there exists a.s. an infinite open cluster, whence

$$
P_{p}(B(m) \leftrightarrow \infty) \rightarrow 1 \quad \text { as } \quad m \rightarrow \infty .
$$

We pick $m$ such that

$$
P_{p}(B(m) \leftrightarrow \infty)>1-\left(\frac{1}{3} \eta\right)^{24},
$$

for a reason which will become clear later.

For $n>m$, let $V(n)=\{x \in T(n): x \leftrightarrow B(m)$ in $B(n)\}$. Pick $M$ such that

$$
p P_{p}(B(m) \text { is a seed })>1-\left(\frac{1}{2} \eta\right)^{1 / M} .
$$

We shall assume for simplicity that $2 m+1$ divides $n+1$ (and that $2 m<n$ ), and we partition $T(n)$ into disjoint squares with side-length $2 m$. If $|V(n)| \geq(2 m+1)^{2} M$ then $B(m)$ is joined in $B(n)$ to at least $M$ of these squares. Therefore, by (7.12),

$$
P_{p}(B(m) \leftrightarrow K(m, n) \text { in } B(n))
$$

$$
\begin{aligned}
\text { (7.13) } \quad & \geq\left\{1-\left[1-p P_{p}(B(m) \text { is a seed })\right]^{M}\right\} P_{p}\left(|V(n)| \geq(2 m+1)^{2} M\right) \\
& \geq\left(1-\frac{1}{2} \eta\right) P_{p}\left(|V(n)| \geq(2 m+1)^{2} M\right) .
\end{aligned}
$$

We now bound the last probability from below. Using the symmetries of $\mathbb{L}^{3}$ obtained by reflections in hyperplanes, we see that the face $F(n)$ comprises fou copies of $T(n)$. Now $\partial B(n)$ has six faces, and therefore 24 copies of $T(n)$. By symmetry and the FKG inequality,

$$
\text { (7.14) } \quad P_{p}\left(|U(n)|<24(2 m+1)^{2} M\right) \geq P_{p}\left(|V(n)|<(2 m+1)^{2} M\right)^{24}
$$

where $U(n)=\{x \in \partial B(n): x \leftrightarrow B(m)$ in $B(n)\}$. Now, with $l=24(2 m+1)^{2} M$,

(7.15) $\quad P_{p}(|U(n)|<l) \leq P_{p}(|U(n)|<l, B(m) \leftrightarrow \infty)+P_{p}(B(m) \leftrightarrow \infty)$,

and

$$
\begin{aligned}
P_{p}(|U(n)|<l, B(m) \leftrightarrow \infty) & \leq P_{p}(1 \leq|U(n)|<l) \\
& \leq(1-p)^{-3 l} P_{p}(U(n+1)=\varnothing, U(n) \neq \varnothing) \\
& \rightarrow 0 \quad \text { as } n \rightarrow \infty .
\end{aligned}
$$

(Here we use the fact that $U(n+1)=\varnothing$ if every edge exiting $\partial B(n)$ from $U(n)$ is closed.)

By (7.14)-(7.16) and (7.11),

$$
P_{p}\left(|V(n)|<(2 m+1)^{2} M\right) \leq P_{p}(|U(n)|<l)^{1 / 24} \leq\left(a_{n}+\left(\frac{1}{3} \eta\right)^{24}\right)^{1 / 24}
$$

where $a_{n} \rightarrow 0$ as $n \rightarrow \infty$. We pick $n$ such that

$$
P_{p}\left(|V(n)|<(2 m+1)^{2} M\right) \leq \frac{1}{2} \eta,
$$

and the claim of the lemma follows by (7.13).

Having constructed open paths from $B(m)$ to $K(m, n)$, we shall need to repeat the construction, beginning instead at an appropriate seed in $K(m, n)$. This is problematic, since we have discovered a mixture of information, some of it negative, about the immediate environs of such seeds. In order to overcome the effect of such negative information, we shall work at edge-density $p+\delta$ rather than $p$. In preparation, let $\left(X(e): e \in \mathbb{E}^{d}\right)$ be independent random variables having the uniform distribution on $[0,1]$, and let $\eta_{p}(e)$ be the indicator function that $X(e)<p$; recall Section 2.3. We say that $e$ is $p$-open if $X(e)<p$ and $p$-closed otherwise, and we denote by $P$ the appropriate probability measure.

For any subset $V$ of $\mathbb{Z}^{3}$, we define the exterior boundary $\Delta V$ and exterior edgeboundary $\Delta_{\mathrm{e}} V$ by

$$
\begin{aligned}
\Delta V & =\left\{x \in \mathbb{Z}^{3}: x \notin V, x \sim y \text { for some } y \in V\right\}, \\
\Delta_{\mathrm{e}} V & =\{\langle x, y\rangle: x \in V, y \in \Delta V, x \sim y\} .
\end{aligned}
$$

We write $\mathbb{E}_{V}$ for the set of all edges of $\mathbb{L}^{3}$ joining pairs of vertices in $V$. 
$B(n)$

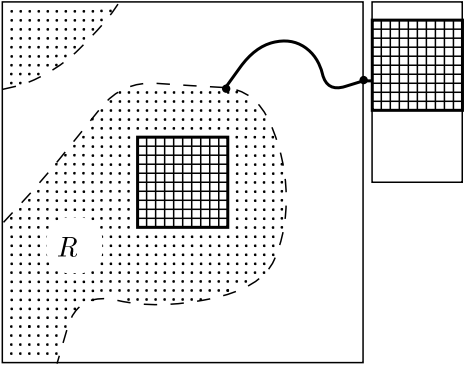

Fig. 7.4. An illustration of Lemma 7.17. The hatched regions are copies of $B(m)$ all of whose edges are $p$-open. The central box $B(m)$ lies within some (dotted) region $R$. Some vertex in $R$ is joined by a path to some
connected to a seed lying on the surface of $B(n)$.

We shall make repeated use of the following lemma ${ }^{5}$, which is illustrated in Figure 7.4

Lemma 7.17. If $\theta(p)>0$ and $\epsilon, \delta>0$, there exist integers $m=m(p, \epsilon, \delta)$ and $n=n(p, \epsilon, \delta)$ such that $2 m<n$ and with the following property. Let $R$ be such that $B(m) \subseteq R \subseteq B(n)$ and $(R \cup \Delta R) \cap T(n)=\varnothing$, and let $\beta: \Delta_{\mathrm{e}} R \cap \mathbb{E}_{B(n)} \rightarrow[0,1-\delta]$. $B(m) \subseteq R \subseteq B(n)$

$G=\{$ there exists a path joining $R$ to $K(m, n)$, this path being $p$-open outside $\Delta_{\mathrm{e}} R$ and $(\beta(e)+\delta)$-open at its unique edge $e$ lying in $\left.\Delta_{\mathrm{e}} R\right\}$,

$H=\left\{e\right.$ is $\beta(e)$-closed for all $\left.e \in \Delta_{\mathrm{e}} R \cap \mathbb{E}_{B(n)}\right\}$.

Then $P(G \mid H)>1-\epsilon$.

Proof. Assume that $\theta(p)>0$, and let $\epsilon, \delta>0$. Pick an integer $t$ so large that

$$
(1-\delta)^{t}<\frac{1}{2} \epsilon
$$

and then choose $\eta(>0)$ such that

$$
\eta<\frac{1}{2} \epsilon(1-p)^{t}
$$

We apply Lemma 7.9 with this value of $\eta$, thereby obtaining integers $m, n$ such that $2 m<n$ and

$$
P_{p}(B(m) \leftrightarrow K(m, n) \text { in } B(n))>1-\eta .
$$

${ }^{5}$ This lemma is basically Lemma 6 of [165], the difference being that [165] was addressed at site percolation. R. Meester and J. Steif have kindly pointed out that, in the case of site percolation, a slightly more general lemma is required than that presented in [165]. The following remarks are directed at the necessary changes to Lemma 6 of [165], and they use the notation of [165]. The proof of the more general lemma is similar to that of the original version. The domain of $\beta$ is $S$ to $K(m, n)$ this path being $p$ - $(n) \backslash T(n(n) \backslash S$ and $(\beta(u)+\delta)$-open at its unique vertex $u \in S\}$. In applying the lemma just after (4.10) of [165], we take $S=\Delta C_{2} \cap B(n)$ (and similarly later).
Let $R$ and $\beta$ satisfy the hypotheses of the lemma. Since any path from $B(m)$ to $K(m, n)$ contains a path from $\partial R$ to $K(m, n)$ using no edges of $\mathbb{E}_{R}$, we have that

$$
P_{p}(\partial R \leftrightarrow K(m, n) \text { in } B(n))>1-\eta .
$$

Let $K \subseteq T(n)$, and let $U(K)$ be the set of edges $\langle x, y\rangle$ of $B(n)$ such that (i) $x \in R, y \notin R$, and

(ii) there is an open path joining $y$ to $K$, using no edges of $\mathbb{E}_{R} \cup \Delta_{\mathrm{e}} R$

We wish to show that $U(K)$ must be large if $P_{p}(\partial R \leftrightarrow K$ in $B(n))$ is large. The argument centres on the fact that every path from $\partial R$ to $K$ passes through $U(K)$; if $U(K)$ is 'small' then there is substantial uncertainty for the occurrence of the event $\{\partial R \leftrightarrow K$ in $B(n)\}$, implying that this event cannot have probability near 1. More rigorously,

$$
\begin{aligned}
P_{p}(\partial R \leftrightarrow K \text { in } B(n)) & =P_{p}(\text { all edges in } U(K) \text { are closed }) \\
& \geq(1-p)^{t} P_{p}(|U(K)| \leq t) .
\end{aligned}
$$

We may apply this with $K=K(m, n)$, since $K(m, n)$ is defined on the set of edges exterior to $B(n)$. Therefore, by (7.21), (7.22), and (7.19),

$(7.23)$

$$
\begin{aligned}
P_{p}(|U(K(m, n))|>t) & \geq 1-(1-p)^{-t} P_{p}(\partial R \leftrightarrow K(m, n) \text { in } B(n)) \\
& \geq 1-(1-p)^{-t} \eta>1-\frac{1}{2} \epsilon .
\end{aligned}
$$

We now couple together the percolation processes with different values of $p$ on the same probability space, as described in Section 2.3 and just prior to the statement of Lemma 7.17. We borrow the notation and results derived above by specialising to the $p$-open edges. Conditional on the set $U=U(K(m, n))$, the values of $X(e)$, for $e \in U$, are independent and uniform on $[0,1]$. Therefore

$$
P(\text { every } e \text { in } U \text { is }(\beta(e)+\delta) \text {-closed, }|U|>t \mid H) \leq(1-\delta)^{t},
$$

whence, using (7.18) and (7.23),

$$
\begin{aligned}
P(\text { some } e \text { in } U \text { is }(\beta(e)+\delta) \text {-open } \mid H) & \geq P(|U|>t \mid H)-(1-\delta)^{t} \\
& =P_{p}(|U|>t)-(1-\delta)^{t} \\
& \geq\left(1-\frac{1}{2} \epsilon\right)-\frac{1}{2} \epsilon,
\end{aligned}
$$

and the lemma is proved.

This completes the two key geometrical lemmas. In moving to the second part of the proof, we shall require a method for comparison of a 'dependent' process and a site percolation process. The argument required at this stage is as follows.

Let $F$ be an infinite connected subset of $\mathbb{L}^{d}$ for which the associated (site) critical probability satisfies $p_{\mathrm{c}}(F$, site $)<1$, and let $\{Z(x): x \in F\}$ be random variables taking values in $[0,1]$. We construct a connected subset of $F$ in the following recursive 
manner. Let $e(1), e(2), \ldots$ be a fixed ordering of the edges of the graph induced by $F$. Let $x_{1} \in F$, and define the ordered pair $S_{1}=\left(A_{1}, B_{1}\right)$ of subsets of $F$ by

$$
S_{1}= \begin{cases}\left(\left\{x_{1}\right\}, \varnothing\right) & \text { if } Z\left(x_{1}\right)=1 \\ \left(\varnothing,\left\{x_{1}\right\}\right) & \text { if } Z\left(x_{1}\right)=0 .\end{cases}
$$

Having defined $S_{1}, S_{2}, \ldots, S_{t}=\left(A_{t}, B_{t}\right)$, for $t \geq 1$, we define $S_{t+1}$ as follows. Let $f$ be the earliest edge in the fixed ordering of the $e(i)$ with the property that one endvertex, $x_{t+1}$ say, lies in $A_{t}$ and the other endvertex lies outside $A_{t} \cup B_{t}$. Then we declare

$$
S_{t+1}= \begin{cases}\left(A_{t} \cup\left\{x_{t+1}\right\}, B_{t}\right) & \text { if } Z\left(x_{t+1}\right)=1, \\ \left(A_{t}, B_{t} \cup\left\{x_{t+1}\right\}\right) & \text { if } Z\left(x_{t+1}\right)=0 .\end{cases}
$$

If no such edge $f$ exists, we declare $S_{t+1}=S_{t}$. The sets $A_{t}, B_{t}$ are non-decreasing and we set $A_{\infty}=\lim _{t \rightarrow \infty} A_{t}, B_{\infty}=\lim _{t \rightarrow \infty} B_{t}$. Think about $A_{\infty}$ as the 'occupied cluster' at $x_{1}$, and $B_{\infty}$ as its external boundary.

Lemma 7.24. Suppose there exists a constant $\gamma$ such that $\gamma>p_{\mathrm{c}}(F$, site $)$ and

$$
P\left(Z\left(x_{t+1}\right)=1 \mid S_{1}, S_{2}, \ldots, S_{t}\right) \geq \gamma \text { for all } t .
$$

Then $P\left(\left|A_{\infty}\right|=\infty\right)>0$.

We omit a formal proof of this lemma (but see [165]). Informally, (7.25) implies that, uniformly in the past history, the chance of extending $A_{t}$ exceeds the critical value of a supercritical site percolation process on $F$. Therefore $A_{\infty}$ stochastically dominates the open cluster at $x_{1}$ of a supercritical site percolation cluster. The latter cluster is infinite with strictly positive probability, whence $P\left(\left|A_{\infty}\right|=\infty\right)>0$.

Having established the three basic lemmas, we turn to the construction itself Recall the notation and hypotheses of Theorem 7.8. Let $0<\eta<p_{\mathrm{c}}$, and choose

$$
p=p_{\mathrm{c}}+\frac{1}{2} \eta, \quad \delta=\frac{1}{12} \eta, \quad \epsilon=\frac{1}{24}\left(1-p_{\mathrm{c}}(F, \text { site })\right) .
$$

Note that $p_{\mathrm{c}}(F$, site $)<1$ since by assumption $p_{\mathrm{c}}(F)=p_{\mathrm{c}}(F$, bond $)<1$ (cf. Theorem 5.13). Since $p>p_{\mathrm{c}}$, we have that $\theta(p)>0$, and we apply Lemma 7.17 with the above $\epsilon, \delta$ to find corresponding integers $m, n$. We define $N=m+n+1$, and we shall define a process on the blocks of $\mathbb{Z}^{3}$ having side-length $2 N$.

Consider the set $\left\{4 N x: x \in \mathbb{Z}^{d}\right\}$ of vertices, and the associated boxes $B_{x}(N)=$ $\left\{4 N x+B(N): x \in \mathbb{Z}^{d}\right\}$; these boxes we call site-boxes. A pair $B_{x}(N), B_{y}(N)$ of site-boxes is deemed adjacent if $x$ and $y$ are adjacent in $\mathbb{L}^{d}$. Adjacent site-boxes are linked by bond-boxes, i.e., boxes $N z+B(N)$ for $z \in \mathbb{Z}^{d}$ exactly one component of which is not divisible by 4 . If this exceptional component of $z$ is even, the box $N z+B(N)$ is called a half-way box. See Figure 7.5.

We shall examine site-boxes one by one, declaring each to be 'occupied' or 'unoccupied' according to the existence (or not) of certain open paths. Two properties of this construction will emerge.

(a) For each new site-box, the probability that it is occupied exceeds the critical probability of a certain site percolation process. This will imply that, with strictly positive probability, there is an infinite occupied path of site-boxes.

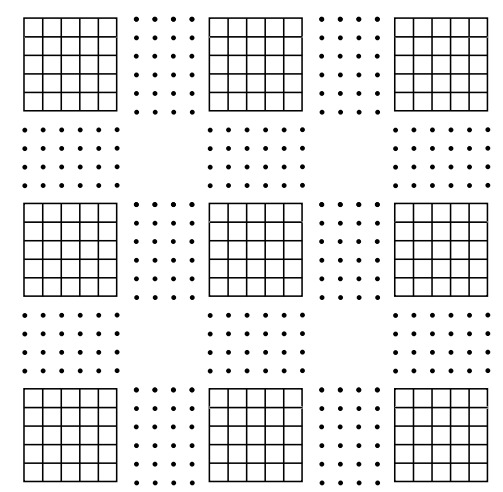

Fig. 7.5. The hatched squares are site-boxes, and the dotted squares are half-way boxes. Each box has side-length $2 N$.

(b) The existence of this infinite occupied path necessarily entails an infinite open path of $\mathbb{L}^{d}$ lying within some restricted region.

The site-boxes will be examined in sequence, the order of this sequence being random, and depending on the past history of the process. Thus, the renormalisation is 'dynamic' rather than 'static'.

As above, let $F$ be an infinite connected subset of $\mathbb{Z}^{d}$; we shall assume for neatness that $F$ contains the origin 0 (otherwise, translate $F$ accordingly). As above, le $e(1), e(2), \ldots$ be a fixed ordering of the edges joining vertices in $F$. We shall examine the site-boxes $B_{x}(N)$, for $x \in F$, and determine their states. This we do according to the algorithm sketched before Lemma 7.24 , for appropriate random variables $Z(x)$ to be described next.

We begin at the origin, with the site-box $B_{0}(N)=B(N)$. Once we have explained what is involved in determining the state of $B_{0}(N)$, most of the work will have been done. (The event $\left\{B_{0}(N)\right.$ is occupied $\}$ is sketched in Figure 7.7.)

Note that $B(m) \subseteq B(N)$, and say that 'the first step is successful' if every edge in $B(m)$ is $p$-open, which is to say that $B(m)$ is a 'seed'. (Recall that $p$ and othe parameters are given in (7.26).) At this stage we write $E_{1}$ for the set of edges of $B(m)$.

In the following sequential algorithm, we shall construct an increasing sequence $E_{1}, E_{2}, \ldots$ of edge-sets. At each stage $k$, we shall acquire information about the values of $X(e)$ for certain $e \in \mathbb{E}^{3}$ (here, the $X(e)$ are independent uniform $[0,1]$ valued random variables, as usual). This information we shall record in the form 'each $e$ is $\beta_{k}(e)$-closed and $\gamma_{k}(e)$-open' for suitable functions $\beta_{k}, \gamma_{k}: \mathbb{E}^{3} \rightarrow[0,1]$ satisfying

(7.27) $\quad \beta_{k}(e) \leq \beta_{k+1}(e), \quad \gamma_{k}(e) \geq \gamma_{k+1}(e), \quad$ for all $e \in \mathbb{E}^{3}$ 


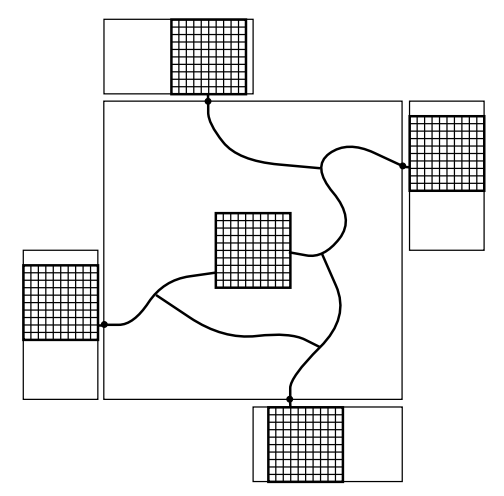

Fig. 7.6. An illustration of the first two steps in the construction of the event $\{0$ is occupied $\}$, when these steps are successful. Each hatched square is a seed.

Having constructed $E_{1}$, above, we set

$$
\begin{aligned}
& \beta_{1}(e)=0 \quad \text { for all } e \in \mathbb{E}^{3}, \\
& \gamma_{1}(e)= \begin{cases}p & \text { if } e \in E_{1}, \\
1 & \text { otherwise }\end{cases}
\end{aligned}
$$

Since we are working with edge-sets $E_{j}$ rather than with vertex-sets, it will be useful to have some corresponding notation. Two edges $e, f$ are called adjacent, written $e \approx f$, if they have exactly one common endvertex. This adjacency relation defines a graph. Paths in this graph are said to be $\alpha$-open if $X(e)<\alpha$ for all $e$ lying in the path. The exterior edge-boundary $\Delta_{\mathrm{e}} E$ of an edge-set $E$ is the set of all edges $f \in \mathbb{E}^{3} \backslash E$ such that $f \approx e$ for some $e \in E$.

For $j=1,2,3$ and $\sigma= \pm$, let $L_{j}^{\sigma}$ be an automorphism of $\mathbb{L}^{3}$ which preserves the origin and maps $e_{1}=(1,0,0)$ onto $\sigma e_{j}$; we insist that $L_{1}^{+}$is the identity. We now define $E_{2}$ as follows. Consider the set of all paths $\pi$ lying within the region

$$
B_{1}^{\prime}=B(n) \cup\left\{\bigcup_{\substack{1 \leq j \leq 3 \\ \sigma= \pm 土}} L_{j}^{\sigma}(T(m, n))\right\}
$$

such that

(a) the first edge $f$ of $\pi$ lies in $\Delta_{\mathrm{e}} E_{1}$ and is $\left(\beta_{1}(f)+\delta\right)$-open, and

(b) all other edges lie outside $E_{1} \cup \Delta_{\mathrm{e}} E_{1}$ and are $p$-open.

We define $E_{2}=E_{1} \cup F_{1}$ where $F_{1}$ is the set of all edges in the union of such paths $\pi$. We say that 'the second step is successful' if, for each $j=1,2,3$ and $\sigma= \pm$, there is an edge in $E_{2}$ having an endvertex in $K_{j}^{\sigma}(m, n)$, where

$$
K_{j}^{\sigma}(m, n)=\left\{z \in L_{j}^{\sigma}(T(n)):\left\langle z, z+\sigma e_{j}\right\rangle \text { is } p \text {-open, and } z+\sigma e_{j}\right. \text { lies }
$$$$
\text { in some seed lying within } \left.L_{j}^{\sigma}(T(m, n))\right\} \text {. }
$$

The corresponding event is illustrated in Figure 7.6.
Next we estimate the probability that the second step is successful, conditional on the first step being successful. Let $G$ be the event that there exists a path in $B(n) \backslash B(m)$ from $\partial B(m)$ to $K(m, n)$, every edge $e$ of which is $p$-open off $\Delta_{\mathrm{e}} E_{1}$ and whose unique edge $f$ in $\Delta_{\mathrm{e}} E_{1}$ is $\left(\beta_{1}(f)+\delta\right)$-open. We write $G_{j}^{\sigma}$ for the corresponding event with $K(m, n)$ replaced by $L_{j}^{\sigma}(K(m, n))$. We now apply Lemma 7.17 with $R=B(m)$ and $\beta=\beta_{1}$ to find that

$$
P\left(G_{j}^{\sigma} \mid B(m) \text { is a seed }\right)>1-\epsilon \text { for } j=1,2,3, \sigma= \pm .
$$

Therefore

$$
P\left(G_{j}^{\sigma} \text { occurs for all } j, \sigma \mid B(m) \text { is a seed }\right)>1-6 \epsilon,
$$

so that the second step is successful with conditional probability at least $1-6 \epsilon$

If the second step is successful, then we update the $\beta, \gamma$ functions accordingly, setting

$$
\begin{gathered}
\beta_{2}(e)= \begin{cases}\beta_{1}(e) & \text { if } e \notin \mathbb{E}_{B_{1}^{\prime}}, \\
\beta_{1}(e)+\delta & \text { if } e \in \Delta_{\mathrm{e}} E_{1} \backslash E_{2}, \\
p & \text { if } e \in \Delta_{\mathrm{e}} E_{2} \backslash \Delta_{\mathrm{e}} E_{1}, \\
0 & \text { otherwise, }\end{cases} \\
\gamma_{2}(e)= \begin{cases}\gamma_{1}(e) & \text { if } e \in E_{1}, \\
\beta_{1}(e)+\delta & \text { if } e \in \Delta_{\mathrm{e}} E_{1} \cap E_{2}, \\
p & \text { if } e \in E_{2} \backslash\left(E_{1} \cup \Delta_{\mathrm{e}} E_{1}\right), \\
1 & \text { otherwise }\end{cases}
\end{gathered}
$$

Suppose that the first two steps have been successful. We next aim to link the appropriate seeds in each $L_{j}^{\sigma}(T(m, n))$ to a new seed lying in the bond-box $2 \sigma N e_{j}+B(N)$, i.e., the half-way box reached by exiting the origin in the direction $\sigma e_{j}$. If we succeed with each of the six such extensions, then we terminate this stage of the process, and declare the vertex 0 of the renormalised lattice to be occupied; such success constitutes the definition of the term 'occupied'. See Figure 7.7.

We do not present all the details of this part of the construction, since they are very similar to those already described. Instead we concentrate on describing the basic strategy, and discussing any novel aspects of the construction. First, let $B_{2}=b_{2}+B(m)$ be the earliest seed (in some ordering of all copies of $B(m)$ ) all of whose edges lie in $E_{2} \cap \mathbb{E}_{T(m, n)}$. We now try to extend $E_{2}$ to include a seed lying within the bond-box $2 N e_{1}+B(N)$. Clearly $B_{2} \subseteq N e_{1}+B(N)$. In performing this extension, we encounter a 'steering' problem. It happens (by construction) that all coordinates of $b_{2}$ are positive, implying that $b_{2}+T(m, n)$ is not a subset of $2 N e_{1}+B(N)$. We therefore replace $b_{2}+T(m, n)$ by $b_{2}+T^{*}(m, n)$ where $T^{*}(m, n)$ is given as follows. Instead of working with the 'quadrant' $T(n)$ of the face $F(n)$, we use the set

$$
T^{*}(n)=\left\{x \in \partial B(n): x_{1}=n, x_{j} \leq 0 \text { for } j=2,3\right\} .
$$


61

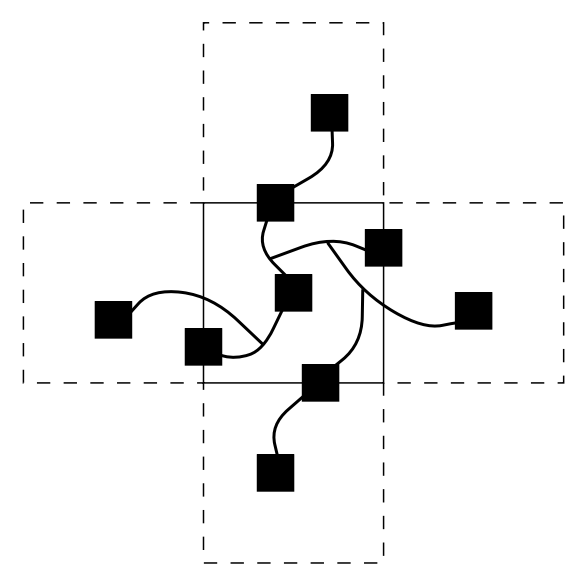

Fig. 7.7. An illustration of the event $\{0$ is occupied $\}$. Each black square is a seed.

We then define

$$
T^{*}(m, n)=\bigcup_{j=1}^{2 m+1}\left\{j e_{1}+T^{*}(n)\right\},
$$

and obtain that $b_{2}+T^{*}(m, n) \subseteq 2 N e_{1}+B(N)$. We now consider the set of all paths $\pi$ lying within the region

$$
B_{2}^{\prime}=b_{2}+\left\{B(n) \cup T^{*}(m, n)\right\}
$$

such that:

(a) the first edge $f$ of $\pi$ lies in $\Delta_{\mathrm{e}} E_{2}$ and is $\left(\beta_{2}(f)+\delta\right)$-open, and

(b) all other edges lie outside $E_{2} \cup \Delta_{\mathrm{e}} E_{2}$ and are $p$-open.

We set $E_{3}=E_{2} \cup F_{2}$ where $F_{2}$ is the set of all edges lying in the union of such paths. We call this step successful if $E_{3}$ contains an edge having an endvertex in the set

$$
b_{2}+K^{*}(m, n)=\left\{z \in b_{2}+T^{*}(m, n):\left\langle z, z+e_{1}\right\rangle \text { is } p \text {-open, } z+e_{1}\right.
$$

$$
\text { lies in some seed lying in } \left.b_{2}+T^{*}(m, n)\right\} \text {. }
$$

Using Lemma 7.17, the (conditional) probability that this step is successful exceeds $1-\epsilon$.

We perform similar extensions in each of the other five directions exiting $B_{0}(N)$. If all are successful, we declare 0 to be occupied. Combining the above estimates of success, we find that

$(7.33)$

$P(0$ is occupied $\mid B(m)$ is a seed $)>(1-6 \epsilon)(1-\epsilon)^{6}>1-12 \epsilon$

$$
=\frac{1}{2}\left(1+p_{\mathrm{c}}(F, \text { site })\right)
$$

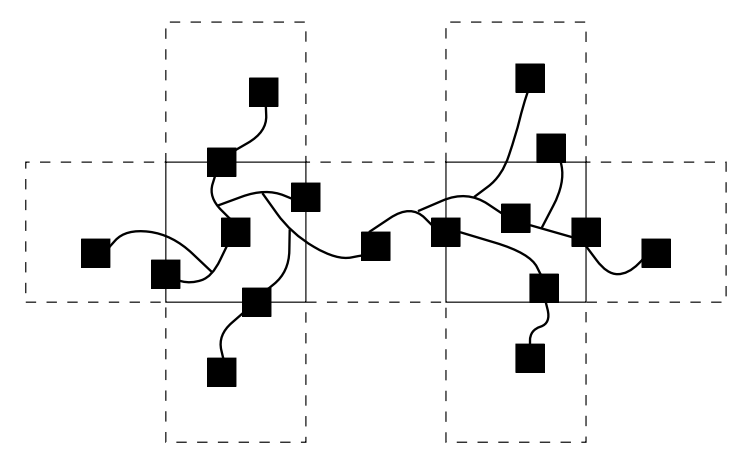
Fig. 7.8. Two adjacent site-boxes both of which are occupied. The construction
began with the left site-box $B_{0}(N)$ and has been extended to the right sitebox $B_{e_{1}}(N)$. The black squares are seeds, as before.

by $(7.26)$.

If 0 is not occupied, we end the construction. If 0 is occupied, then this has been achieved after the definition of a set $E_{8}$ of edges. The corresponding functions $\beta_{8}, \gamma_{8}$ are such that

$$
\beta_{8}(e) \leq \gamma_{8}(e) \leq p+6 \delta \text { for } e \in E_{8} ;
$$

this follows since no edge lies in more than 7 of the copies of $B(n)$ used in the repeated application of Lemma 7.17. Therefore every edge of $E_{8}$ is $(p+\eta)$-open, since $\delta=\frac{1}{12} \eta($ see $(7.26))$

The basic idea has been described, and we now proceed similarly. Assume 0 is occupied, and find the earliest edge $e(r)$ induced by $F$ and incident with the origin; we may assume for the sake of simplicity that $e(r)=\left\langle 0, e_{1}\right\rangle$. We now attempt to link the seed $b_{3}+B(m)$, found as above inside the half-way box $2 N e_{1}+B(N)$, to a seed inside the site-box $4 N e_{1}+B(N)$. This is done in two steps of the earlier kind. Having found a suitable seed inside the new site-box $4 N e_{1}+B(N)$, we attempt to branch-out in the other 5 directions from this site-box. If we succeed in finding seeds in each of the corresponding half-way boxes, then we declare the vertex $e_{1}$ of the renormalised lattice to be occupied. As before, the (conditional) probability that $e_{1}$ is occupied is at least $\frac{1}{2}\left(1+p_{\mathrm{c}}(F\right.$, site $\left.)\right)$, and every edge in the ensuing construction is $(p+\eta)$-open. See Figure 7.8.

Two details arise at this and subsequent stages, each associated with 'steering'. First, if $b_{3}=\left(\alpha_{1}, \alpha_{2}, \alpha_{3}\right)$ we concentrate on the quadrant $T_{\alpha}(n)$ of $\partial B(n)$ defined as the set of $x \in \partial B(n)$ for which $x_{j} \alpha_{j} \leq 0$ for $j=2,3$ (so that $x_{j}$ has the opposite sign to $\alpha_{j}$ ). Having found such a $T_{\alpha}(n)$, we define $T_{\alpha}^{*}(m, n)$ accordingly, and look for paths from $b_{3}+B(m)$ to $b_{3}+T_{*}^{*}(m, n)$. This mechanism guarantes that any the next step.

A further detail arises when branching out from the seed $b^{*}+B(m)$ reached inside $4 N e_{1}+B(N)$. In finding seeds lying in the new half-way boxes abutting 


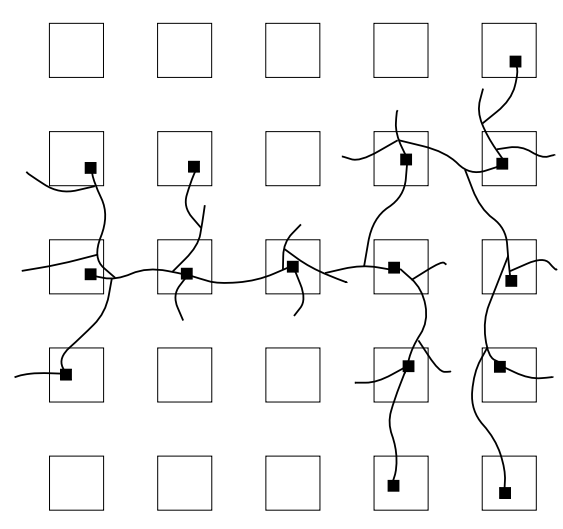

Fig. 7.9. The central seed is $B(m)$, and the connections represent $(p+\eta)$-open path joining seeds within the site-boxes.

$4 N e_{1}+B(N)$, we 'steer away from the inlet branch', by examining seeds lying on the surface of $b^{*}+B(n)$ with the property that the first coordinates of their vertices are not less than that of $b^{*}$. This process guarantees that these seeds have not been examined previously.

We now continue to apply the algorithm presented before Lemma 7.24. At each stage, the chance of success exceeds $\gamma=\frac{1}{2}\left(1+p_{\mathrm{c}}(F\right.$, site $\left.)\right)$. Since $\gamma>p_{\mathrm{c}}(F$, site), we

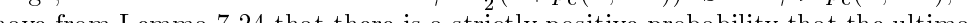
have from Lex set of occupied vertices of $F$ (i.e., renormalised bocks of $\mathbb{L}^{3}$ ) is infinite. Now, o this event, there must exist an infinite $(p+\eta)$-open path of $\mathbb{L}^{3}$ corresponding to the enlargement of $F$. This infinite open path must lie within the enlarged se $4 N F+B(2 N)$, implying that $p_{\mathrm{c}}+\eta \geq p_{\mathrm{c}}(4 N F+B(2 N))$, as required for Theorem 7.9. See Figure 7.9. The proof is complete.

\subsection{Percolation in Half-Spaces}

In the last section, we almost succeeded in proving that $\theta\left(p_{\mathrm{c}}\right)=0$ when $d>3$. The reason for this statement is as follows. Suppose $\theta(p)>0$ and $\eta>0$. There is effectively defined in Section 7.3 an event $A$ living in a finite box $B$ such that

(a) $P_{p}(A)>1-\epsilon$, for some prescribed $\epsilon>0$

(b) the fact (a) implies that $\theta(p+\eta)>0$.

Suppose that we could prove this with $\eta=0$, and that $\theta\left(p_{\mathrm{c}}\right)>0$. Then $P_{p_{\mathrm{c}}}(A)>$ $1-\epsilon$, which implies by continuity that $P_{p^{\prime}}(A)>1-\epsilon$ for some $p^{\prime}<p_{\mathrm{c}}$, and therefore $\theta\left(p^{\prime}\right)>0$ by (b). This would contradict the definition of $p_{\mathrm{c}}$, whence we deduce by contradiction that $\theta\left(p_{\mathrm{c}}\right)=0$

The fact that $\eta$ is strictly positive is vital for the construction, since we need to 'spend some extra money' in order to compensate for negative information acquired earlier in the construction. In a slightly different setting, no extra money is required.
Let $\mathbb{H}=\{0,1, \ldots\} \times \mathbb{Z}^{d-1}$ be a half-space when $d \geq 3$, and write $p_{\mathrm{c}}(\mathbb{H})$ for its critical probability. It follows from Theorem 7.8 that $p_{\mathrm{c}}(\mathbb{H})=p_{\mathrm{c}}$, since $\mathbb{H}$ contains slabs of all thicknesses. Let

$$
\theta_{\mathbb{H}}(p)=P_{p}(0 \leftrightarrow \infty \text { in } \mathbb{H}) .
$$

Theorem 7.35. We have that $\theta_{\mathbb{H}}\left(p_{\mathrm{c}}\right)=0$.

The proof is not presented here, but may be found in $[49,50]$. It is closely related to that presented in Section 7.3, but with some crucial differences. The construction of blocks is slightly more complicated, owing to the lack of symmetry of $\mathbb{H}$, but there are compensating advantages of working in a half-space. For amusement, we present in Figure 7.10 two diagrams (relevant to the argument of [50]) depicting the necessary constructions.

As observed in Section 3.3, such a conclusion for half-spaces has a striking implication for the conjecture that $\theta\left(p_{\mathrm{c}}\right)=0$. If $\theta\left(p_{\mathrm{c}}\right)>0$, then there exists a.s. a unique infinite open cluster in $\mathbb{Z}^{d}$, which is a.s. partitioned into (only) finite clusters by any division of $\mathbb{Z}^{d}$ into two half-spaces.

\subsection{Percolation Probability}

Although the methods of Chapter 6 were derived primarily in order to study $s u b$ critical percolation, they involve a general inequality of wider use, namely

$$
g_{\pi}^{\prime}(n) \geq g_{\pi}(n)\left(\frac{n}{\sum_{i=0}^{n} g_{\pi}(i)}-1\right)
$$

where $g_{\pi}(n)=P_{\pi}\left(0 \leftrightarrow \partial S_{n}\right)$; see equations (3.10) and (3.18) in Section 6. We argue loosely as follows. Clearly $g_{\pi}(n) \rightarrow \theta(\pi)$ as $n \rightarrow \infty$, whence (cross your fingers here)

$$
\theta^{\prime}(\pi) \geq \theta(\pi)\left(\frac{1}{\theta(\pi)}-1\right)
$$

$$
\theta^{\prime}(\pi)+\theta(\pi) \geq 1 .
$$

Integrate this over the interval $\left(p_{\mathrm{c}}, p\right)$ to obtain

$$
\theta(p) e^{p}-\theta\left(p_{\mathrm{c}}\right) e^{p_{\mathrm{c}}} \geq e^{p}-e^{p_{\mathrm{c}}}, \quad p_{\mathrm{c}} \leq p,
$$

whence it is an easy exercise to show that

$$
\theta(p)-\theta\left(p_{\mathrm{c}}\right) \geq a\left(p-p_{\mathrm{c}}\right), \quad p_{\mathrm{c}} \leq p,
$$

for some positive constant $a$. The above argument may be made rigorous.

Differential inequalities of the type above are used widely in percolation and disordered systems. 


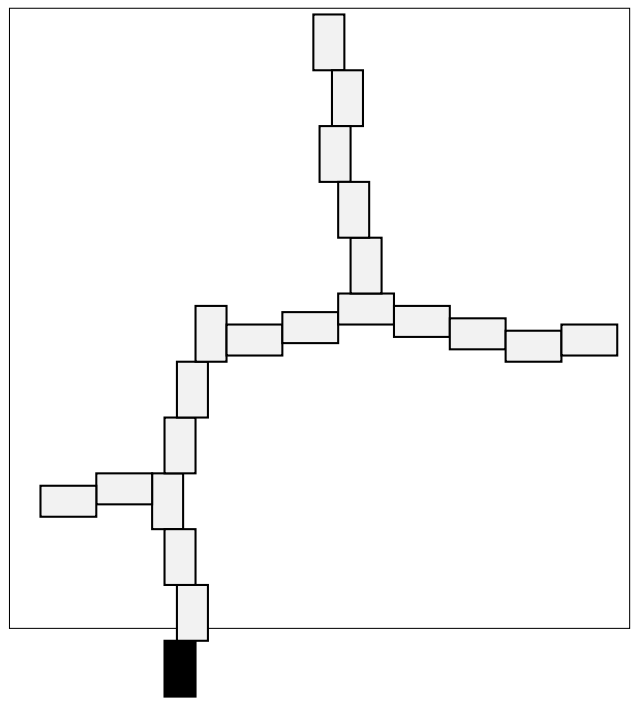

7.6 Cluster-Size Distribution

When $p<p_{\mathrm{c}}$, the tail of the cluster-size $|C|$ decays exponentially. Exponential decay is not correct when $p>p_{\mathrm{c}}$, but rather 'stretched exponential decay'.

Theorem 7.37. Suppose $p_{\mathrm{c}}<p<1$. There exist positive constants $\alpha(p), \beta(p)$ such that, for all $n$

(7.38) $\quad \exp \left(-\alpha n^{(d-1) / d}\right) \leq P_{p}(|C|=n) \leq \exp \left(-\beta n^{(d-1) / d}\right)$.

See $[\mathrm{G}]$ for a proof of this theorem. The reason for the power $n^{(d-1) / d}$ is roughly as follows. It is thought that a large finite cluster is most likely created as a cluster of compact shape, all of whose boundary edges are closed. Now, if a ball has volume $n$, then its surface area has order $n^{(d-1) / d}$. The price paid for having a surface all of whose edges are closed is $(1-p)^{m}$ where $m$ is the number of such edges. By the above remark, $m$ should have order $n^{(d-1) / d}$, as required for (7.38).

It is believed that the limit

$$
\gamma(p)=\lim _{n \rightarrow \infty}\left\{-\frac{1}{n^{(d-1) / d}} \log P_{p}(|C|=n)\right\}
$$

exists, but no proof is known.

Much more is known in two dimensions than for general $d$. The size and geometry of large finite clusters have been studied in detail in [38], where it was shown that such clusters may be approximated by the so called 'Wulff shape'. This work includes a proof of the existence of the limit in (7.39) when $d=2$.

Fig. 7.10. Illustrations of the block construction for the proof of Theorem 735 presented in [50] The grey regions contain open paths joining the black 'inlet' to the three 'outlets'. The fundamental building block is a rectangle rather than a square. We have no control of the aspect ratio of this rectangle, and consequently two cases with somewhat different geometries need to be considered. Compare with Figure 7.7. 


\section{CRITICAL PERCOLATION}

8.1 Percolation Probability

The next main open question is to verify the following.

Conjecture 8.1. We have that $\theta\left(p_{\mathrm{c}}\right)=0$

This is known to hold when $d=2$ (using results of Harris [181], see Theorem 9.1) and for sufficiently large values of $d$ (by work of Hara and Slade [178, 179]), currently for $d \geq 19$. The methods of Hara and Slade might prove feasible for values of $d$ as small as 6 or 7 , but not for smaller $d$. Some new idea is needed for the general conclusion. As remarked in Section 7.4, we need to rule out the remaining theoretical possibility that there is an infinite cluster in $\mathbb{Z}^{d}$ when $p=p_{\mathrm{c}}$, but no infinite cluster in any half-space.

\subsection{CRitical Exponents}

Macroscopic functions, such as the percolation probability, have a singularity at $p=p_{\mathrm{c}}$, and it is believed that there is 'power law behaviour' at and near this singularity. The nature of the singularity is supposed to be canonical, in that it is expected to have certain general features in common with phase transitions in other physical systems. These features are sometimes referred to as 'scaling theory' and they relate to 'critical exponents'

There are two sets of critical exponents, arising firstly in the limit as $p \rightarrow p_{\mathrm{c}}$, and secondly in the limit over increasing distances when $p=p_{\mathrm{c}}$. We summarise the notation in Table 7.1.

The asymptotic relation $\approx$ should be interpreted loosely (perhaps via logarithmic asymptotics). The radius of $C$ is defined by $\operatorname{rad}(C)=\max \{n: 0 \leftrightarrow \partial B(n)\}$. The limit as $p \rightarrow p_{\mathrm{c}}$ should be interpreted in a manner appropriate for the function in question (for example, as $p \downarrow p_{\mathrm{c}}$ for $\theta(p)$, but as $p \rightarrow p_{\mathrm{c}}$ for $\kappa(p)$ ).

There are eight critical exponents listed in Table 7.1 , denoted $\alpha, \beta, \gamma, \delta, \nu, \eta, \rho, \Delta$ but there is no general proof of the existence of any of these exponents.

\subsection{SCALING THEORY}

In general, the eight critical exponents may be defined for phase transitions in a quite large family of physical systems. However, it is not believed that they are independent variables, but rather that they satisfy the following:

(8.2) Scaling relations

$$
\begin{aligned}
2-\alpha & =\gamma+2 \beta=\beta(\delta+1), \\
\Delta & =\delta \beta, \\
\gamma & =\nu(2-\eta),
\end{aligned}
$$

\begin{tabular}{|c|c|c|c|}
\hline \multicolumn{2}{|r|}{ Function } & Behaviour & Exponent \\
\hline $\begin{array}{l}\text { percolation } \\
\text { probability }\end{array}$ & $\theta(p)=P_{p}(|C|=\infty)$ & $\theta(p) \approx\left(p-p_{\mathrm{c}}\right)^{\beta}$ & $\beta$ \\
\hline $\begin{array}{c}\text { truncated } \\
\text { mean cluster size }\end{array}$ & $\chi^{\mathrm{f}}(p)=E_{p}(|C| ;|C|<\infty)$ & $\chi^{\mathrm{f}}(p) \approx\left|p-p_{\mathrm{c}}\right|^{-\gamma}$ & $\gamma$ \\
\hline $\begin{array}{c}\text { number of } \\
\text { clusters per vertex }\end{array}$ & $\kappa(p)=E_{p}\left(|C|^{-1}\right)$ & $\kappa^{\prime \prime \prime}(p) \approx\left|p-p_{c}\right|^{-1-\alpha}$ & $\alpha$ \\
\hline cluster moments & $\chi_{k}^{\mathrm{f}}(p)=E_{p}\left(|C|^{k} ;|C|<\infty\right)$ & $\frac{\chi_{k+1}^{\mathrm{f}}(p)}{\chi_{k}^{\mathrm{f}}(p)} \approx\left|p-p_{\mathrm{c}}\right|^{-\Delta}, k \geq 1$ & $\Delta$ \\
\hline correlation length & $\xi(p)$ & $\xi(p) \approx\left|p-p_{\mathrm{c}}\right|^{-\nu}$ & $\nu$ \\
\hline \multicolumn{2}{|c|}{ cluster volume } & $P_{p_{\mathrm{c}}}(|C|=n) \approx n^{-1-1 / \delta}$ & $\delta$ \\
\hline \multicolumn{2}{|c|}{ cluster radius } & $P_{p_{\mathrm{c}}}(\operatorname{rad}(C)=n) \approx n^{-1-1 / \rho}$ & $\rho$ \\
\hline \multicolumn{2}{|c|}{ connectivity function } & $P_{p_{\mathrm{c}}}(0 \leftrightarrow x) \approx\|x\|^{2-d-\eta}$ & $\eta$ \\
\hline
\end{tabular}

Table 7.1. Eight functions and their critical exponent

and, when $d$ is not too large, the

(8.3) Hyperscaling relations

$$
\begin{aligned}
d \rho & =\delta+1, \\
2-\alpha & =d \nu .
\end{aligned}
$$

The upper critical dimension is the largest value $d_{c}$ such that the hyperscaling relations hold for $d \leq d_{\mathrm{c}}$. It is believed that $d_{\mathrm{c}}=6$ for percolation.

There is no general proof of the validity of the scaling and hyperscaling relations, although certain things are known when $d=2$ and for large $d$.

In the context of percolation, there is an analytical rationale behind the scaling relations, namely the 'scaling hypotheses' that

$$
\begin{aligned}
P_{p}(|C|=n) & \sim n^{-\sigma} f\left(n / \xi(p)^{\tau}\right) \\
P_{p}(0 \leftrightarrow x,|C|<\infty) & \sim\|x\|^{2-d-\eta} g(\|x\| / \xi(p))
\end{aligned}
$$

in the double limit as $p \rightarrow p_{\mathrm{c}}, n \rightarrow \infty$, and for some constants $\sigma, \tau, \eta$ and functions $f, g$. Playing loose with rigorous mathematics, the scaling relations may be derived from these hypotheses. Similarly, the hyperscaling relations may be shown to be not too unreasonable, at least when $d$ is not too large. For further discussion, see $[\mathrm{G}]$ 
We make some further points.

Universality. It is believed that the numerical values of critical exponents depend only on the value of $d$, and are independent of the particular percolation model.

Two dimensions. When $d=2$, perhaps

$$
\alpha=-\frac{2}{3}, \quad \beta=\frac{5}{36}, \quad \gamma=\frac{43}{18}, \quad \delta=\frac{91}{5}, \ldots
$$

Large dimension. When $d$ is sufficiently large (actually, $d \geq d_{c}$ ) it is believed that the critical exponents are the same as those for percolation on a tree (the 'meanfield model'), namely $\delta=2, \gamma=1, \nu=\frac{1}{2}, \rho=\frac{1}{2}$, and so on (the other exponents are found to satisfy the scaling relations). Using the first hyperscaling relation, this supports the contention that $d_{\mathrm{c}}=6$. Such statements are known to hold for $d \geq 19$; see $[178,179]$ and Section 8.5 .

\subsection{Rigorous Resutes}

Open challenges include to prove:

- the existence of critical exponents,

- universality,

- the scaling relations,

- the conjectured values when $d=2$,

- the conjectured values when $d \geq 6$.

Progress towards these goals has been slender, but positive. Most is known in the case of large $d$, see the next section. For sufficiently large $d$, exact values are known for many exponents, namely the values from percolation on a regular tree. When $d=2$, Kesten $[204,205]$ has proved that, if two critical exponents exist, then certain others do also, and certain scaling relations are valid. However, the provocative case when $d=3$ is fairly open terrain.

Certain partial results are known in generality, yielding inequalities in situations where one expects (asymptotic) equalities. For example, it is known that $\beta<1$, if $\beta$ exists (cf. (7.36)). In similar vein, we have that $\gamma \geq 1$ and $\delta>2$ for all $d$.

\subsection{Mean-Field Theory}

The expression 'mean-field' permits several interpretations depending on context. A narrow interpretation of the term 'mean-field theory' for percolation involves trees rather than lattices. For percolation on a regular tree, it is quite easy to perform exact calculations of many quantities, including the numerical values of critical exponents. That is, $\delta=2, \gamma=1, \nu=\frac{1}{2}, \rho=\frac{1}{2}$, and other exponents are given according to the scaling relations (8.2); see [G], Section 8.1 .

Turning to percolation on $\mathbb{L}^{d}$, it is known that the critical exponents agree with those of a regular tree when $d$ is sufficiently large. In fact, this is believed to hold if and only if $d \geq 6$, but progress so far assumes that $d \geq 19$. In the following theorem, taken from [179], we write $f(x) \simeq g(x)$ if there exist positive constants $c_{1}, c_{2}$ such that $c_{1} f(x) \leq g(x) \leq c_{2} f(x)$ for all $x$ close to a limiting value.
Theorem 8.4. If $d \geq 19$ then

$$
\begin{array}{rlrl}
\theta(p) & \simeq\left(p-p_{\mathrm{c}}\right)^{1} & & \text { as } p \downarrow p_{\mathrm{c}}, \\
\chi(p) & \simeq\left(p_{\mathrm{c}}-p\right)^{-1} \quad \text { as } p \uparrow p_{\mathrm{c}}, \\
\xi(p) & \simeq\left(p_{\mathrm{c}}-p\right)^{-\frac{1}{2}} \quad \text { as } p \uparrow p_{\mathrm{c}}, \\
\frac{\chi_{k+1}^{\mathrm{f}}(p)}{\chi_{k}^{\mathrm{f}}(p)} & \simeq\left(p_{\mathrm{c}}-p\right)^{-2} & & \text { as } p \uparrow p_{\mathrm{c}}, \text { for } k \geq 1 .
\end{array}
$$

Note the strong form of the asymptotic relation $\simeq$, and the identification of the critical exponents $\beta, \gamma, \Delta, \nu$. The proof of Theorem 8.4 centres on a property known as the 'triangle condition'. Define

$$
T(p)=\sum_{x, y \in \mathbb{Z}^{d}} P_{p}(0 \leftrightarrow x) P_{p}(x \leftrightarrow y) P_{p}(y \leftrightarrow 0),
$$

and introduce the following condition,

(8.10) Triangle condition:

$T\left(p_{\mathrm{c}}\right)<\infty$.

The triangle condition was introduced by Aizenman and Newman [27], who showed that it implied that $\chi(p) \simeq\left(p_{\mathrm{c}}-p\right)^{-1}$ as $p \uparrow p_{\mathrm{c}}$. Subsequently other authors showed that the triangle condition implied similar asymptotics for other quantities. It was Hara and Slade [178] who verified the triangle condition for large $d$, exploiting a technique known as the 'lace expansion'.

We present no full proof of Theorem 8.4 here, pleading two reasons. First, such a proof would be long and complicated. Secondly, we are unable to do better tha is already contained in the existing literature (see $[178,179]$ ). Instead, we (nearly) prove the above Aizenman-Newman result (equation (8.6) above), namely that the triangle condition implies that $\chi(p) \sim\left(p_{c}-p\right)^{-1}$ as $p \uparrow p_{c}$, then we present a very We begin with a lemma.

Lemma 8.11. Let $\tau_{p}(u, v)=P_{p}(u \leftrightarrow v)$, and

$$
Q(a, b)=\sum_{v, w \in \mathbb{Z}^{d}} \tau_{p}(a, v) \tau_{p}(v, w) \tau_{p}(w, b) \quad \text { for } a, b \in \mathbb{Z}^{d} .
$$

Then $Q$ is a positive-definite form, in that

$$
\sum_{a, b} f(a) Q(a, b) \overline{f(b)} \geq 0
$$

for all suitable functions $f: \mathbb{Z}^{d} \rightarrow \mathbb{C}$.

Proof. We have that

$$
\begin{aligned}
\sum_{a, b} f(a) Q(a, b) \overline{f(b)} & =\sum_{v, w} g(v) \tau_{p}(v, w) \overline{g(w)} \\
& =E_{p}\left(\sum_{v, w} g(v) 1_{\{v \leftrightarrow w\}} \overline{g(w)}\right) \\
& =E_{p}\left(\sum_{C}\left|\sum_{x \in C} g(x)\right|^{2}\right)
\end{aligned}
$$


71

where $g(v)=\sum_{a} f(a) \tau_{p}(a, v)$, and the penultimate summation is over all open clusters $C$.

We note the consequence of Lemma 8.11, that

$(8.12)$

$$
Q(a, b)^{2} \leq Q(a, a) Q(b, b)=T(p)^{2}
$$

by Schwarz's inequality.

Theorem 8.13. If $d \geq 2$ and $T\left(p_{\mathrm{c}}\right)<\infty$ then

$$
\chi(p) \simeq\left(p_{\mathrm{c}}-p\right)^{-1} \quad \text { as } p \uparrow p_{\mathrm{c}} .
$$

Proof. This is taken from [27]; see also [G] and [179]. The following sketch is incomplete in one important regard, namely that, in the use of Russo's formula, one should first restrict oneself to a finite region $\Lambda$, and later pass to the limit as $\Lambda \uparrow \mathbb{Z}^{d}$; we omit the details of this.

Write $\tau_{p}(u, v)=P_{p}(u \leftrightarrow v)$ as before, so that

$$
\chi(p)=\sum_{x \in \mathbb{Z}^{d}} \tau_{p}(0, x) .
$$

By (ab)use of Russo's formula,

(8.14) $\quad \frac{d \chi}{d p}=\frac{d}{d p} \sum_{x \in \mathbb{Z}^{d}} \tau_{p}(0, x)=\sum_{x \in \mathbb{Z}^{d}} \sum_{e \in \mathbb{E}^{d}} P_{p}(e$ is pivotal for $\{0 \leftrightarrow x\})$.

If $e=\langle a, b\rangle$ is pivotal for $\{0 \leftrightarrow x\}$, then one of the events $\{0 \leftrightarrow a\} \circ\{b \leftrightarrow x\}$ and $\{0 \leftrightarrow b\} \circ\{a \leftrightarrow x\}$ occurs. Therefore, by the BK inequality,

$$
\begin{aligned}
\frac{d \chi}{d p} & \leq \sum_{x} \sum_{e=\langle a, b\rangle}\left\{\tau_{p}(0, a) \tau_{p}(b, x)+\tau_{p}(0, b) \tau_{p}(a, x)\right\} \\
& =\sum_{e=\langle a, b\rangle}\left\{\tau_{p}(0, a)+\tau_{p}(0, b)\right\} \chi(p)=2 d \chi(p)^{2} .
\end{aligned}
$$

This inequality may be integrated, to obtain that

$$
\frac{1}{\chi\left(p_{1}\right)}-\frac{1}{\chi\left(p_{2}\right)} \leq 2 d\left(p_{2}-p_{1}\right) \text { for } p_{1} \leq p_{2}
$$

Take $p_{1}=p<p_{\mathrm{c}}$ and $p_{2}>p_{\mathrm{c}}$, and allow the limit $p_{2} \downarrow p_{\mathrm{c}}$, thereby obtaining that (8.16)

$$
\chi(p) \geq \frac{1}{2 d\left(p_{\mathrm{c}}-p\right)} \quad \text { for } p<p_{\mathrm{c}} .
$$

In order to obtain a corresponding lower bound for $\chi(p)$, we need to obtain a lower bound for (8.14). Let $e=\langle a, b\rangle$ in (8.14), and change variables $(x \mapsto x-a)$ in the summation to obtain that

$$
\frac{d \chi}{d p}=\sum_{x, y} \sum_{|u|=1} P_{p}\left(0 \leftrightarrow x, u \leftrightarrow y \text { off } C_{u}(x)\right)
$$

where the second summation is over all unit vectors $u$ of $\mathbb{Z}^{d}$. The (random set) $C_{u}(x)$ is defined as the set of all points joined to $x$ by open paths not using $\langle 0, u\rangle$.

In the next lemma, we have a strictly positive integer $R$, and we let $B=B(R)$. The set $C_{B}(x)$ is the set of all points reachable from $x$ along open paths using no vertex of $B$.

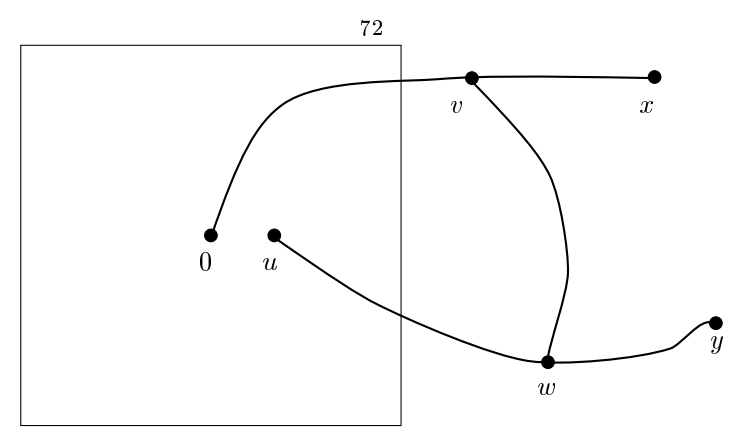

Fig. 8.1. If $0 \leftrightarrow x, u \leftrightarrow y$, and $x \leftrightarrow y$ off $B$, then there exist $v, w \notin B$ such that there are disjoint open paths from $x$ to $v$, from $v$ to 0 , from $v$ to $w$, from $w$ to $u$, and from $w$ to $y$.

Lemma 8.18. Let $u$ be a unit vector. We have that

$P_{p}\left(0 \leftrightarrow x, u \leftrightarrow y\right.$ off $\left.C_{u}(x)\right) \geq \alpha(p) P_{p}\left(0 \leftrightarrow x, u \leftrightarrow y\right.$ off $\left.C_{B}(x)\right)$,

where $\alpha(p)=\{\min (p, 1-p)\}^{2 d(2 R+1)^{d}}$

Proof of Lemma 8.18. Define the following events,

$$
\begin{aligned}
& E=\left\{0 \leftrightarrow x, u \leftrightarrow y \text { off } C_{u}(x)\right\}, \\
& F=\left\{0 \leftrightarrow x, u \leftrightarrow y \text { off } C_{B}(x)\right\}, \\
& G=\left\{B \cap C(x) \neq \varnothing, B \cap C(y) \neq \varnothing, C_{B}(x) \cap C_{B}(y)=\varnothing\right\},
\end{aligned}
$$

noting that $E \subseteq F \subseteq G$. Now

$$
P_{p}(E)=P_{p}(E \mid G) P_{p}(G) \geq P_{p}(E \mid G) P_{p}(F) .
$$

The event $G$ is independent of all edges lying in the edge-set $\mathbb{E}_{B}$ of $B$. Also, for any $\omega \in G$, there exists a configuration $\omega_{B}=\omega_{B}(\omega)$ for the edges in $\mathbb{E}_{B}$ such that the composite configuration $\left(\omega\right.$ off $\mathbb{E}_{B}$, and $\omega_{B}$ on $\mathbb{E}_{B}$ ) lies in $E$. Since $\mathbb{E}_{B}$ is finite, and $P_{p}\left(\omega_{B}\right) \geq \alpha(p)$ whatever the choice of $\omega_{B}$, we have that $P_{p}(E \mid G) \geq \alpha(p)$, and the conclusion of the lemma follows.

The event $F$ in (8.19) satisfies

$$
F=\{0 \leftrightarrow x, u \leftrightarrow y\} \backslash\{0 \leftrightarrow x, u \leftrightarrow y, x \leftrightarrow y \text { off } B\},
$$

whence, by the FKG inequality,

$$
\begin{aligned}
P_{p}(F) & =P_{p}(0 \leftrightarrow x, u \leftrightarrow y)-P_{p}(0 \leftrightarrow x, u \leftrightarrow y, x \leftrightarrow y \text { off } B) \\
& \geq \tau_{p}(0, x) \tau_{p}(u, y)-P_{p}(0 \leftrightarrow x, u \leftrightarrow y, x \leftrightarrow y \text { off } B) .
\end{aligned}
$$

To bound the last term, we use the BK inequality. Glancing at Figure 8.1, we see that, if $0 \leftrightarrow x, u \leftrightarrow y$, and $x \leftrightarrow y$ off $B$, then there exist $v, w \notin B$ such that there 
are disjoint open paths from $x$ to $v$, from $v$ to 0 , from $v$ to $w$, from $w$ to $u$, and from $w$ to $y$. Applying the BK inequality,

$$
P_{p}(F) \geq \tau_{p}(0, x) \tau_{p}(u, y)-\sum_{v, w \notin B} \tau_{p}(x, v) \tau_{p}(v, 0) \tau_{p}(v, w) \tau_{p}(w, u) \tau_{p}(w, y) .
$$

Now sum over $x$ and $y$ to obtain via (8.17) and Lemma 8.18 that

$$
\frac{d \chi}{d p} \geq 2 d \alpha(p) \chi^{2}\left(1-\sup _{|u|=1} \sum_{v, w \notin B} \tau_{p}(0, v) \tau_{p}(v, w) \tau_{p}(w, u)\right) .
$$

Now, by (8.12),

$$
\sum_{v, w} \tau_{p}(0, v) \tau_{p}(v, w) \tau_{p}(w, u) \leq T(p) \quad \text { for all } u .
$$

Assuming that $T\left(p_{\mathrm{c}}\right)<\infty$, we may choose $B=B(R)$ sufficiently large that

$$
\frac{d \chi}{d p} \geq 2 d \alpha(p) \chi^{2}\left(1-\frac{1}{2}\right) \quad \text { for } p \leq p_{\mathrm{c}} .
$$

Integrate this, as for (8.15), to obtain that

$$
\chi(p) \leq \frac{1}{\alpha^{\prime}\left(p_{\mathrm{c}}-p\right)} \quad \text { for } p \leq p_{\mathrm{c}}
$$

where $\alpha^{\prime}=\alpha^{\prime}(p)$ is strictly positive and continuous for $0<p<1$ (and we have used the fact (6.7) that $\left.\chi\left(p_{\mathrm{c}}\right)=\infty\right)$.

Finally we discuss the verification of the triangle condition $T\left(p_{\mathrm{c}}\right)<\infty$. This has been proved for large $d$ (currently $d \geq 19$ ) by Hara and Slade [176, 177, 178, 179, 180], and is believed to hold for $d \geq 7$. The corresponding condition for a 'spread-out' percolation model, having large but finite-range links rather than nearest-neighbour only, is known to hold for $d>6$.

The proof that $T\left(p_{\mathrm{c}}\right)<\infty$ is long and technical, and is to be found in [178]; since the present author has no significant improvement on that version, the details are not given here. Instead, we survey briefly the structure of the proof.

The triangle function (8.9) involves convolutions, and it is therefore natural to introduce the Fourier transform of the connectivity function $\tau_{p}(x, y)=P_{p}(x \leftrightarrow y)$.

More generally, if $f: \mathbb{Z}^{d} \rightarrow \mathbb{R}$ is summable, we define

$$
\widehat{f}(\theta)=\sum_{x \in \mathbb{Z}^{d}} f(x) e^{i \theta \cdot x}, \quad \text { for } \theta=\left(\theta_{1}, \ldots, \theta_{d}\right) \in[-\pi, \pi]^{d},
$$

where $\theta \cdot x=\sum_{j=1}^{d} \theta_{j} x_{j}$. If $f$ is symmetric (i.e., $f(x)=f(-x)$ for all $x$ ), then $\widehat{f}$ is real.

We have now that

(8.23) $\quad T(p)=\sum_{x, y} \tau_{p}(0, x) \tau_{p}(x, y) \tau_{p}(y, 0)=(2 \pi)^{-d} \int_{[-\pi, \pi]^{d}} \widehat{\tau}_{p}(\theta)^{3} d \theta$.
The proof that $T\left(p_{\mathrm{c}}\right)<\infty$ involves an upper bound on $\widehat{\tau}_{p}$, namely the so called infra-red bound

$(8.24)$

$$
\widehat{\tau}_{p}(\theta) \leq \frac{c(p)}{|\theta|^{2}}
$$

where $|\theta|=\sqrt{\theta \cdot \theta}$. It is immediate via (8.23) that the infra-red bound (8.24) implie that $T(p)<\infty$. Also, if $(8.24)$ holds for some $c(p)$ which is uniformly bounded for $p<p_{\mathrm{c}}$, then $T\left(p_{\mathrm{c}}\right)=\lim _{p \uparrow p_{\mathrm{c}}} T(p)<\infty$.

It is believed that

$$
\widehat{\tau}_{p}(\theta) \simeq \frac{1}{|\theta|^{2-\eta}} \quad \text { as }|\theta| \rightarrow 0
$$

where $\eta$ is the critical exponent given in the table of Section 8.2.

Theorem 8.26 (Hara-Slade [178]). There exists D satisfying $D>6$ such that, if $d \geq D$, then

$$
\widehat{\tau}_{p}(\theta) \leq \frac{c(p)}{|\theta|^{2}}
$$

for some $c(p)$ which is uniformly bounded for $p<p_{\mathrm{c}}$. Also $T\left(p_{\mathrm{c}}\right)<\infty$.

The proof is achieved by establishing and using the following three facts:

(a) $T(p)$ and

$$
W(p)=\sum_{x \in \mathbb{Z}^{d}}|x|^{2} \tau_{p}(0, x)^{2}
$$

are continuous for $p \leq p_{\mathrm{c}}$;

(b) there exist constants $k_{T}$ and $k_{W}$ such that

$$
T(p) \leq 1+\frac{k_{T}}{d}, \quad W(p) \leq \frac{k_{W}}{d}, \quad \text { for } p \leq \frac{1}{2 d} ;
$$

(c) for large $d$, and for $p$ satisfying $(2 d)^{-1} \leq p<p_{\mathrm{c}}$, we have that

$$
T(p) \leq 1+\frac{3 k_{T}}{d}, \quad W(p) \leq \frac{3 k_{W}}{d}, \quad p \leq \frac{3}{2 d}
$$

whenever

$$
T(p) \leq 1+\frac{4 k_{T}}{d}, \quad W(p) \leq \frac{4 k_{W}}{d}, \quad p \leq \frac{4}{2 d} .
$$

Fact (a) is a consequence of the continuity of $\tau_{p}$ and monotone convergence Fact (b) follows by comparison with a simpler model (the required comparison is successful for sufficiently small $p$, namely $p \leq(2 d)^{-1}$ ). Fact (c) is much harder to prove, and it is here that the 'lace expansion' is used. Part (c) implies that there is a 'forbidden region' for the pairs $(p, T(p))$ and $(p, W(p))$; see Figure 8.2. Since $T$ and $W$ are finite for small $p$, and continuous up to $p_{\mathrm{c}}$, part (c) implies that

$$
T\left(p_{\mathrm{c}}\right) \leq 1+\frac{3 k_{T}}{d}, \quad W\left(p_{\mathrm{c}}\right) \leq \frac{3 k_{W}}{d}, \quad p_{\mathrm{c}} \leq \frac{3}{2 d} .
$$




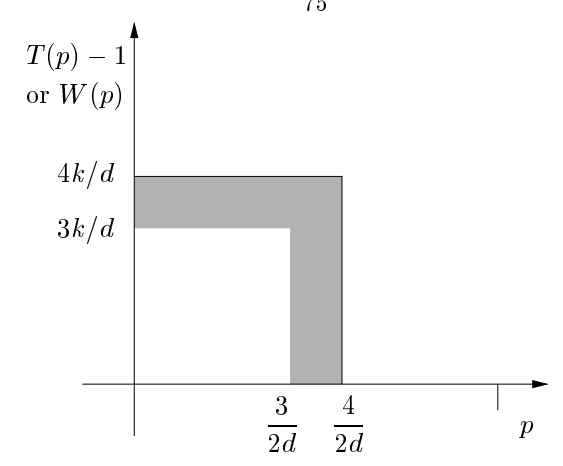

Fig. 8.2. There is a 'forbidden region' for the pairs $(p, T(p)-1)$ and $(p, W(p))$, namely the shaded region in this figure. The quantity $k$ denotes $k_{T}$ or $k_{W}$ as appropriate

The infra-red bound emerges in the proof of (c), of which there follows an extremely brief account

We write $x \Leftrightarrow y$, and say that $x$ is 'doubly connected' to $y$, if there exist two edge-disjoint open paths from $x$ to $y$. We express $\tau_{p}(0, x)$ in terms of the 'doubly connected' probabilities $\delta_{p}(u, v)=P_{p}(u \Leftrightarrow v)$. In doing so, we encounter formulae involving convolutions, which may be treated by taking transforms. At the first stage, we have that

$$
\{0 \leftrightarrow x\}=\{0 \Leftrightarrow x\} \cup\left\{\bigcup_{\langle u, v\rangle}\{0 \Leftrightarrow(u, v) \leftrightarrow x\}\right\}
$$

where $\{0 \Leftrightarrow(u, v) \leftrightarrow x\}$ represents the event that $\langle u, v\rangle$ is the 'first pivotal edge' for the event $\{0 \leftrightarrow x\}$, and that 0 is doubly connected to $u$. (Similar but more complicated events appear throughout the proof.) Therefore

$$
\tau_{p}(0, x)=\delta_{p}(0, x)+\sum_{\langle u, v\rangle} P_{p}(0 \Leftrightarrow(u, v) \leftrightarrow x) .
$$

Now, with $A(0, u ; v, x)=\left\{v \leftrightarrow x\right.$ off $\left.C_{\langle u, v\rangle}(0)\right\}$,

$$
\begin{aligned}
P_{p}(0 \Leftrightarrow(u, v) \leftrightarrow x) & =p P_{p}(0 \Leftrightarrow u, A(0, u ; v, x)) \\
& =p \delta_{p}(0, u) \tau_{p}(v, x)-p E_{p}\left(1_{\{0 \Leftrightarrow u\}}\left\{\tau_{p}(v, x)-1_{A(0, u ; v, x)}\right\}\right)
\end{aligned}
$$

whence, by (8.27),

$$
\tau_{p}(0, x)=\delta_{p}(0, x)+\delta_{p} \star(p I) \star \tau_{p}(x)-R_{p, 0}(0, x)
$$

where $\star$ denotes convolution, $I$ is the nearest-neighbour function $I(u, v)=1$ if and only if $u \sim v$, and $R_{p, 0}$ is a remainder.
Equation (8.28) is the first step of the lace expansion, In the second step, the remainder $R_{p, 0}$ is expanded similarly, and so on. Such further expansions yield the lace expansion: if $p<p_{\mathrm{c}}$ then

$$
\tau_{p}(0, x)=h_{p, N}(0, x)+h_{p, N} \star(p I) \star \tau_{p}(x)+(-1)^{N+1} R_{p, N}(0, x)
$$

for appropriate remainders $R_{p, N}$, and where

$$
h_{p, N}(0, x)=\delta_{p}(0, x)+\sum_{j=1}^{N}(-1)^{j} \Pi_{p, j}(0, x)
$$

and the $\Pi_{p, n}$ are appropriate functions (see Theorem 4.2 of [179]) involving nested expectations of quantities related to 'double connections'.

We take Fourier transforms of (8.29), and solve to obtain that

$$
\widehat{\tau}_{p}=\frac{\widehat{\delta}_{p}+\sum_{j=1}^{N}(-1)^{j} \widehat{\Pi}_{p, j}+(-1)^{N+1} \widehat{R}_{p, N}}{1-p \widehat{I} \widehat{\delta}_{p}-p \widehat{I} \sum_{j=1}^{N}(-1)^{j} \widehat{\Pi}_{p, j}}
$$

The convergence of the lace expansion, and the consequent validity of this formula for $\widehat{\tau}_{p}$, is obtained roughly as follows. First, one uses the BK inequality to derive bounds for the $\delta_{p}, \Pi_{p, j}, R_{p, j}$ in terms of the functions $T(p)$ and $W(p)$. These bounds then imply bounds for the corresponding transforms. In this way, one may obtain a conclusion which is close to point (c) stated above. 


\section{PERCOLATION IN TWO DIMENSIONS}

9.1 The Critical Probability is $\frac{1}{2}$

The famous exact calculation for bond percolation on $\mathbb{L}^{2}$ is the following, proved originally by Kesten [200]. The proof given here is taken from [G].

Theorem 9.1. The critical probability of bond percolation on $\mathbb{Z}^{2}$ equals $\frac{1}{2}$. Furthermore, $\theta\left(\frac{1}{2}\right)=0$.

Proof. Zhang discovered a beautiful proof that $\theta\left(\frac{1}{2}\right)=0$, using only the uniqueness of the infinite cluster. Set $p=\frac{1}{2}$. Let $T(n)$ be the box $T(n)=[0, n]^{2}$, and find $N$ sufficiently large that

$$
P_{\frac{1}{2}}(\partial T(n) \leftrightarrow \infty)>1-\frac{1}{8^{4}} \quad \text { for } n \geq N .
$$

We set $n=N+1$. Writing $A^{1}, A^{\mathrm{r}}, A^{\mathrm{t}}, A^{\mathrm{b}}$ for the (respective) events that the left, right, top, bottom sides of $T(n)$ are joined to $\infty$ off $T(n)$, we have by the FKG inequality that

$$
\begin{aligned}
P_{\frac{1}{2}}(T(n) \leftrightarrow \infty) & =P_{\frac{1}{2}}\left(\overline{A^{1}} \cap \overline{A^{\mathrm{r}}} \cap \overline{A^{\mathrm{t}}} \cap \overline{A^{\mathrm{b}}}\right) \\
& \geq P_{\frac{1}{2}}\left(\overline{A^{1}}\right) P\left(\overline{A^{\mathrm{r}}}\right) P\left(\overline{A^{\mathrm{t}}}\right) P\left(\overline{A^{\mathrm{b}}}\right) \\
& =P_{\frac{1}{2}}\left(\overline{A^{\mathrm{g}}}\right)^{4}
\end{aligned}
$$

by symmetry, for $\mathrm{g}=1, \mathrm{r}, \mathrm{t}, \mathrm{b}$. Therefore

$$
P_{\frac{1}{2}}\left(A^{\mathrm{g}}\right) \geq 1-\left(1-P_{\frac{1}{2}}(T(n) \leftrightarrow \infty)\right)^{1 / 4}>\frac{7}{8}
$$

Now we move to the dual box, with vertex set $T(n)_{\mathrm{d}}=\left\{x+\left(\frac{1}{2}, \frac{1}{2}\right): 0 \leq\right.$ $\left.x_{1}, x_{2}<n\right\}$. Let $A_{\mathrm{d}}^{\mathrm{l}}, A_{\mathrm{d}}^{\mathrm{r}}, A_{\mathrm{d}}^{\mathrm{t}}, A_{\mathrm{d}}^{\mathrm{b}}$ denote the (respective) events that the left, right, top, bottom sides of $T(n)_{\mathrm{d}}$ are joined to $\infty$ by a closed dual path off $T(n)_{\mathrm{d}}$. Since each edge of the dual is closed with probability $\frac{1}{2}$, we have that

$$
P_{\frac{1}{2}}\left(A_{\mathrm{d}}^{\mathrm{g}}\right)>\frac{7}{8} \quad \text { for } \mathrm{g}=1, \mathrm{r}, \mathrm{t}, \mathrm{b} .
$$

Consider the event $A=A^{\mathrm{l} \cap} A^{\mathrm{r}} \cap A_{\mathrm{d}}^{\mathrm{t}} \cap A_{\mathrm{d}}^{\mathrm{b}}$, and see Figure 9.1. Clearly $P_{\frac{1}{2}}(\bar{A}) \leq \frac{1}{2}$, so that $P_{1}(A) \geq \frac{1}{2}$. However, on $A$, either $\mathbb{L}^{2}$ has two infinite open clusters, or its dual has two infinite closed clusters. Each event has probability 0 , a contradiction. We deduce that $\theta\left(\frac{1}{2}\right)=0$, implying that $p_{\mathrm{c}} \geq \frac{1}{2}$.

Next we prove that $p_{\mathrm{c}} \leq \frac{1}{2}$. Suppose instead that $p_{\mathrm{c}}>\frac{1}{2}$, so that

$$
P_{\frac{1}{2}}(0 \leftrightarrow \partial B(n)) \leq e^{-\gamma n} \quad \text { for all } n,
$$

for some $\gamma>0$. Let $S(n)$ be the graph with vertex set $\left\{x \in \mathbb{Z}^{2}: 0 \leq x_{1} \leq n+1,0 \leq\right.$ $\left.x_{2} \leq n\right\}$ and edge set containing all edges inherited from $\mathbb{L}^{2}$ except those in either

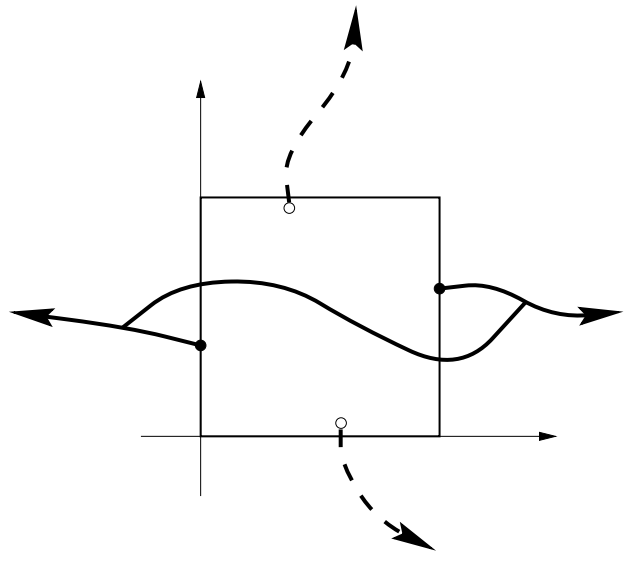

Fig. 9.1. The left and right sides of the box are joined to infinity by open paths of the primal lattice, and the top and bottom sides are joined to infinity by closed dual paths. Using the uniqueness of the infinite open cluster, the two open paths must be oined. This forces the existence of two disjoint infinite closed clusters in the dual.

the left side or the right side of $S(n)$. Denote by $A$ the event that there is an open path joining the left side and right side of $S(n)$. Using duality, if $A$ does not occur then the top side of the dual of $S(n)$ is joined to the bottom side by a closed dual path. Since the dual of $S(n)$ is isomorphic to $S(n)$, and since $p=\frac{1}{2}$, it follows that $P_{\frac{1}{2}}(A)=\frac{1}{2}$. See Figure 9.2. However, using (9.2),

$$
P_{\frac{1}{2}}(A) \leq(n+1) e^{-\gamma n},
$$

a contradiction for large $n$. We deduce that $p_{\mathrm{c}} \leq \frac{1}{2}$.

9.2 RSW TECHNOLOGY

Substantially more is known about the phase transition in two dimensions than in higher dimensions. The main reason for this lies in the fact that geometrical constraints force the intersection of certain paths in two dimensions, whereas they can avoid one another in three dimensions. Path-intersection properties play a central role in two dimensions, whereas in higher dimensions we have to rely on the more complicated Grimmett-Marstrand construction of Section 7.3.

A basic tool in two dimensions is the RSW lemma, which was discovered independently by Russo [326] and Seymour-Welsh [331]. Consider the rectangle $B(k l, l)=[-l,(2 k-1) l] \times[-l, l]$, a rectangle of side-lengths $2 k l$ and $2 l$; note that $B(l, l)=B(l)$. We write $\operatorname{LR}(l)$ for the event that $B(l)$ is crossed from left to right by an open path, and $\mathrm{O}(l)$ for the event that there is an open circuit of the annulus $A(l)=B(3 l) \backslash B(l)$ containing the origin in its interior. 


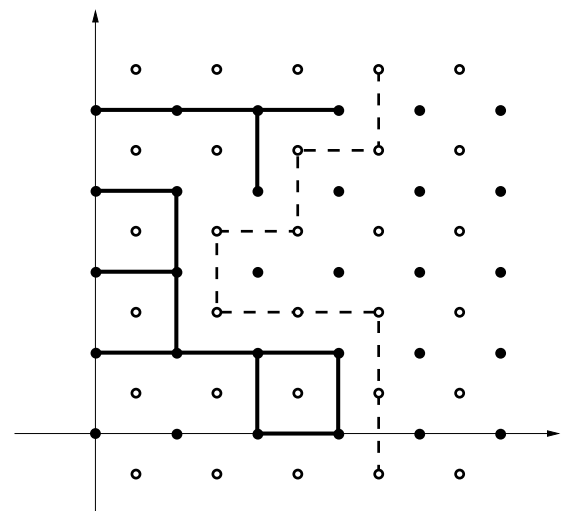

Fig. 9.2. If there is no open left-right crossing of $S(n)$, then there must be a closed

Theorem 9.3 (RSW Lemma). If $P_{p}(\operatorname{LR}(l))=\tau$ then

$$
P_{p}(\mathrm{O}(l)) \geq\left\{\tau(1-\sqrt{1-\tau})^{4}\right\}^{12} .
$$

When $p=\frac{1}{2}$, we have from self-duality that $P_{\frac{1}{2}}(\operatorname{LR}(l)) \geq \frac{1}{4}$ for $l \geq 1$, whence

$$
\text { (9.4) } \quad P_{\frac{1}{2}}(\mathrm{O}(l)) \geq 2^{-24}\left(1-\frac{\sqrt{3}}{2}\right)^{48} \quad \text { for } l \geq 1 \text {. }
$$

We refer the reader to $[\mathrm{G}]$ for a proof of the RSW lemma. In common with almost every published proof of the lemma ([331] is an exception, possibly amongst others), the proof given in $[\mathrm{G}]$ contains a minor error. Specifically, the event $G$ below (9.80) on page 223 is not increasing, and therefore we may not simply use the FKG inequality at (9.81). Instead, let $A_{\pi}$ be the event that the path $\pi$ is open. Then, in the notation of $[\mathrm{G}]$,

$$
\begin{aligned}
P_{\frac{1}{2}}\left(\operatorname{LR}\left(\frac{3}{2} l, l\right)\right) & \geq P_{\frac{1}{2}}\left(N^{+} \cap\left(\bigcup_{\pi \in \mathcal{T}^{-}}\left[A_{\pi} \cap M_{\pi}^{-}\right]\right)\right) \\
& \geq P_{\frac{1}{2}}\left(N^{+}\right) P_{\frac{1}{2}}\left(\bigcup_{\pi}\left[A_{\pi} \cap M_{\pi}^{-}\right]\right) \\
& \geq P_{\frac{1}{2}}\left(N^{+}\right) \sum_{\pi} P_{\frac{1}{2}}\left(L_{\pi} \cap M_{\pi}^{-}\right) \\
& \geq P_{\frac{1}{2}}\left(N^{+}\right)(1-\sqrt{1-\tau}) \sum_{\pi} P_{\frac{1}{2}}\left(L_{\pi}\right) \quad \text { by FKG }(9.84) \\
& =P_{\frac{1}{2}}\left(N^{+}\right)(1-\sqrt{1-\tau}) P_{\frac{1}{2}}\left(L^{-}\right)
\end{aligned}
$$

There are several applications of the RSW lemma, of which we present one.

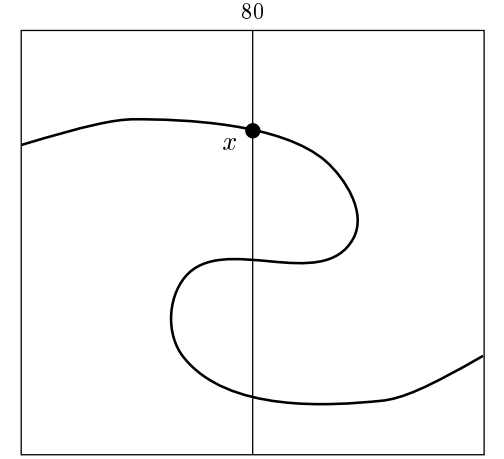

Fig. 9.3. If there is an open left-right crossing of the box, then there must exis in the centre which is connected disjointly to the left and right sides.

Theorem 9.5. There exist constants A, $\alpha$ satisfying $0<A, \alpha<\infty$ such that

$$
\frac{1}{2} n^{-1 / 2} \leq P_{\frac{1}{2}}(0 \leftrightarrow \partial B(n)) \leq A n^{-\alpha} .
$$

Similar power-law estimates are known for other macroscopic quantities at and near the critical point $p_{\mathrm{c}}=\frac{1}{2}$. In the absence of a proof that quantities have 'power-type' singularities near the critical point, it is reasonable to look for upper and lower bounds of the appropriate type. As a general rule, one such bound is and lower bounds of the appropriate type. As a general rule, one such bound is that $\left.\theta(p)-\theta\left(p_{\mathrm{c}}\right) \geq a\left(p-p_{\mathrm{c}}\right)\right)$. The complementary bound is harder, and is generally unavailable at the moment when $d \geq 3$ (but $d$ is not too large).

Proof. Let $R(n)=[0,2 n] \times[0,2 n-1]$, and let $\operatorname{LR}(n)$ be the event that $R(n)$ is traversed from left to right by an open path. We have by self-duality that $P_{\frac{1}{2}}(\operatorname{LR}(n))=\frac{1}{2}$. On the event $\operatorname{LR}(n)$, there exists a vertex $x$ with $x_{1}=n$ such that $x \leftrightarrow x+\partial B(n)$ by two disjoint open paths. See Figure 9.3. Therefore

$$
\frac{1}{2}=P_{\frac{1}{2}}(\operatorname{LR}(n)) \leq \sum_{k=0}^{2 n-1} P_{\frac{1}{2}}\left(A_{k} \circ A_{k}\right) \leq 2 n P_{\frac{1}{2}}(0 \leftrightarrow \partial B(n))^{2}
$$

where $A_{k}=\{(n, k) \leftrightarrow(n, k)+\partial B(n)\}$, and we have used the BK inequality. This provides the lower bound in $(9.6)$.

For the upper bound, we have from (9.4) that $P_{\frac{1}{2}}(\mathrm{O}(l)) \geq \xi$ for all $l$, where $\xi>0$. Now, on the event $\{0 \leftrightarrow \partial B(n)\}$, there can be no closed dual circuit surrounding the origin and contained within $B(n)$. In particular, no dual annulus of the form $B\left(3^{r+1}\right) \backslash B\left(3^{r}\right)+\left(\frac{1}{2}, \frac{1}{2}\right)$, for $0 \leq r<\log _{3} n-1$, can contain such a closed circuit. Therefore

$$
P_{\frac{1}{2}}(0 \leftrightarrow \partial B(n)) \leq(1-\xi)^{\log _{3} n-2}
$$

as required for the upper bound in (9.6). 
9.3 CONFormal Invariance

We concentrate on bond percolation in two dimensions with $p=p_{\mathrm{c}}=\frac{1}{2}$. With $S(n)=[0, n+1] \times[0, n]$, we have by self-duality that

(9.8) $\quad P_{\frac{1}{2}}(S(n)$ traversed from left to right by open path $)=\frac{1}{2}$

for all $n$. Certainly $\mathbb{L}^{2}$ must contain long open paths, but no infinite paths (since $\theta\left(\frac{1}{2}\right)=0$ ). One of the features of (hypothetical) universality is that the chances of long-range connections (when $p=p_{\mathrm{c}}$ ) should be independent of the choice of lattice structure. In particular, local deformations of space should, within limits, not affect such probabilities. One family of local changes arises by local rotations and dilations, and particularly by applying a conformally invariant mapping to $\mathbb{R}^{2}$. and dilations, and particularly by applying a conformally invariant mapping to $\mathbb{R}$. to be explored, invariant under conformal maps of $\mathbb{R}^{2}$. (See [8] for an account of conformal maps.)

Such a hypothesis may be formulated, and investigated numerically. Such a programme has been followed by Langlands, Pouliot, and Saint-Aubin [231] and Aizenman [10], and their results support the hypothesis. In this summary, we refer to bond percolation on $\mathbb{L}^{2}$ only, although such conjectures may be formulated for y two-dimensional percolation model.

We begin with a concrete conjecture concerning crossing probabilities. Let $B(k l, l)$ be a $2 k l$ by $2 l$ rectangle, and let $\operatorname{LR}(k l, l)$ be the event that $B(k l, l)$ is traversed between its opposite sides of length $2 l$ by an open path, as in Section 9.2. It is not difficult to show, using (9.8), that

$$
P_{\frac{1}{2}}(\operatorname{LR}(l, l)) \rightarrow \frac{1}{2} \quad \text { as } l \rightarrow \infty,
$$

and it is reasonable to conjecture that the limit

$$
\lambda_{k}=\lim _{l \rightarrow \infty} P_{\frac{1}{2}}(\operatorname{LR}(k l, l))
$$

exists for all $0<k<\infty$. By self-duality, we have that $\lambda_{k}+\lambda_{k^{-1}}=1$ if the $\lambda_{k}$ exist. It is apparently difficult to establish the limit in (9.9).

In [231] we see a generalisation of this conjecture which is fundamental for a Monte Carlo approach to conformal invariance. Take a simple closed curve $C$ in the plane, and arcs $\alpha_{1}, \alpha_{2}, \ldots, \alpha_{m}, \beta_{1}, \ldots, \beta_{m}$, as well as arcs $\gamma_{1}, \gamma_{2}, \ldots, \gamma_{n}, \delta_{1}, \ldots, \delta_{n}$, of $C$. For a dilation factor $r$, define

$$
\pi_{r}(G)=P\left(r \alpha_{i} \leftrightarrow r \beta_{i}, r \gamma_{i} \leftrightarrow r \delta_{i}, \text { for all } i, \text { in } r C\right)
$$

where $P=P_{p_{\mathrm{c}}}$ and $G$ denotes the collection $\left(C ; \alpha_{i}, \beta_{i} ; \gamma_{i}, \delta_{i}\right)$.
Conjecture 9.11. The following limit exists:

$$
\pi(G)=\lim _{r \rightarrow \infty} \pi_{r}(G)
$$

Some convention is needed in order to make sense of (9.10), arising from the fact that $r C$ lives in the plane $\mathbb{R}^{2}$ rather than on the lattice $\mathbb{L}^{2}$; this poses no major problem. Conjecture (9.9) is a special case of $(9.11)$, with $C=B(k, 1)$, and $\alpha_{1}, \beta_{1}$ being the left and right sides of the box.

Let $\phi: \mathbb{R}^{2} \rightarrow \mathbb{R}^{2}$ be a reasonably smooth function. The composite object $G=$ $\left(C ; \alpha_{i}, \beta_{i} ; \gamma_{i}, \delta_{i}\right)$ has an image under $\phi$, namely $\phi G=\left(\phi C ; \phi \alpha_{i}, \phi \beta_{i} ; \phi \gamma_{i}, \phi \delta_{i}\right)$, which itself corresponds to an event concerning the existence or non-existence of certain open paths. If we believe that crossing probabilities are not affected (as $r \rightarrow \infty$, in $(9.10)$ ) by local dilations and rotations, then it becomes natural to formulate conjecture of invariance under conformal maps [10,231].

Conjecture 9.12 (Conformal Invariance). For all $G=\left(C ; \alpha_{i}, \beta_{i} ; \gamma_{i}, \delta_{i}\right)$, we have that $\pi(\phi G)=\pi(G)$ for any $\phi: \mathbb{R}^{2} \rightarrow \mathbb{R}^{2}$ which is bijective on $C$ and conformal on its interior.

Lengthy computer simulations, reported in [231], support this conjecture. Particularly stimulating evidence is provided by a formula known as Cardy's formula [87]. By following a sequence of transformations of models, and applying ideas of conformal field theory, Cardy was led to an explicit formula for crossing probabilities between two sub-intervals of a simple closed curve $C$.

Let $C$ be a simple closed curve, and let $z_{1}, z_{2}, z_{3}, z_{4}$ be four points on $C$ in clockwise order. There is a conformal map $\phi$ on the interior of $C$ which maps to the unit disc, taking $C$ to its circumference, and the points $z_{i}$ to the points $w_{i}$. Ther are many such maps, but the cross-ratio of such maps,

$$
u=\frac{\left(w_{4}-w_{3}\right)\left(w_{2}-w_{1}\right)}{\left(w_{3}-w_{1}\right)\left(w_{4}-w_{2}\right)},
$$

is a constant satisfying $0 \leq u \leq 1$ (we think of $z_{i}$ and $w_{i}$ as points in the complex plane). We may parametrise the $w_{i}$ as follows: we may assume that

$$
w_{1}=e^{i \theta}, \quad w_{2}=e^{-i \theta}, \quad w_{3}=-e^{i \theta}, \quad w_{4}=-e^{-i \theta}
$$

for some $\theta$ satisfying $0 \leq \theta \leq \frac{\pi}{2}$. Note that $u=\sin ^{2} \theta$. We take $\alpha$ to be the segment of $C$ from $z_{1}$ to $z_{2}$, and $\beta$ the segment from $z_{3}$ to $z_{4}$. Using the hypothesis of conformal invariance, we have that $\pi(G)=\pi(\phi G)$, where $G=(C ; \alpha, \beta ; \varnothing, \varnothing)$, of conformal invariance, we have that $\pi(G)=\pi(\phi G)$, where $G=(C ; \alpha, \beta ; \varnothing, \varnothing)$,
implying that $\pi(G)$ may be expressed as some function $f(u)$, where $u$ is given in implying that $\pi(G)$ may be expressed as some function $f(u)$,
(9.13). Cardy has derived a differential equation for $f$, namely

$$
u(1-u) f^{\prime \prime}(u)+\frac{2}{3}(1-2 u) f^{\prime}(u)=0,
$$

together with the boundary conditions $f(0)=0, f(1)=1$. The solution is a hypergeometric function,

$$
f(u)=\frac{3 \Gamma\left(\frac{2}{3}\right)}{\Gamma\left(\frac{1}{3}\right)^{2}} u^{1 / 3}{ }_{2} F_{1}\left(\frac{1}{3}, \frac{2}{3}, \frac{4}{3} ; u\right) .
$$


Recall that $u=\sin ^{2} \theta$. The derivation is somewhat speculative, but the predictions of the formula may be verified by Monte Carlo simulation (see Figure 3.2 of [231]).

The above 'calculation' is striking. Similar calculations may well be possible for more complicated crossing probabilities than the case treated above. See, for example, $[10,345]$.

In the above formulation, the principle of conformal invariance is expressed in terms of a collection $\{\pi(G)\}$ of limiting 'crossing probabilities'. It would be useful to have a representation of these $\pi(G)$ as probabilities associated with a specific random variable on a specific probability space. Aizenman [10] has made certain proposals about how this might be possible. In his formulation, we observe a bounded region $D_{R}=[0, R]^{2}$, and we shrink the lattice spacing $a$ of bond percolation restricted to this domain. Let $p=p_{c}$, and let $G_{a}$ be the graph of open connections of bond percolation with lattice spacing $a$ on $D_{R}$. By describing $G_{a}$ through the set of Jordan curves describing the realised paths, he has apparently obtained sufficient compactness to imply the existence of weak limits as $a \rightarrow 0$. Possibly there is a unique weak limit, and Aizenman has termed an object sampled according to this limit as the 'web'. The fundamental conjectures are therefore that there is a unique weak limit, and that this limit is conformally invariant.

The quantities $\pi(G)$ should then arise as crossing probabilities in 'web-measure' This geometrical vision may be useful to physicists and mathematicians in understanding conformal invariance.

In one interesting 'continuum' percolation model, conformal invariance may actually be proved rigorously. Drop points $\left\{X_{1}, X_{2}, \ldots\right\}$ in the plane $\mathbb{R}^{2}$ in the manner of a Poisson process with intensity $\lambda$. Now divide $\mathbb{R}^{2}$ into tiles $\left\{T\left(X_{1}\right), T\left(X_{2}\right), \ldots\right\}$, where $T(X)$ is defined as the set of points in $\mathbb{R}^{2}$ which are no further from $X$ than they are from any other point of the Poisson process (this is the 'Voronoi tesselation'). We designate each tile to be open with probability $\frac{1}{2}$ and closed otherwise. This continuum percolation model has a property of self-duality, and it inherits properties of conformal invariance from those of the underlying Poisson point proces See $[11,57]$.

\section{RANDOM WALKS IN RANDOM LABYRINTHS}

10.1 Random Walk on the Infinite Percolation Cluster

It is a classical result that symmetric random walk on $\mathbb{L}^{d}$ is recurrent when $d=2$ but transient when $d \geq 3$ (see [170], pages 188, 266). Three-dimensional space is sufficiently large that a random walker may become lost, whereas two-dimensional space is not. The transience or recurrence of a random walk on a graph $G$ is a crude measure of the 'degree of connectivity' of $G$, a more sophisticated measure being the transition probabilities themselves. In studying the geometry of the infinite open percolation cluster, we may ask whether or not a random walk on this cluster is percolation
recurrent.

Theorem 10.1. Suppose $p>p_{\mathrm{c}}$. Random walk on the (a.s. unique) infinite open cluster is recurrent when $d=2$ and a.s. transient when $d \geq 3$.

This theorem, proved in $[164]^{6}$, follows by a consideration of the infinite open cluster viewed as an electrical network. The relationship between random walks and electrical networks is rather striking, and has proved useful in a number of contexts; see [118].

We denote the (a.s.) unique infinite open cluster by $I=I(\omega)$, whenever it exists. On the graph $I$, we construct a random walk as follows. First, we set $S_{0}=x$ where $x$ is a given vertex of $I$. Given $S_{0}, S_{1}, \ldots, S_{n}$, we specify that $S_{n+1}$ is chosen uniformly from the set of neighbours of $S_{n}$ in $I$, this choice being independent of all earlier choices. We call $\omega$ a transient configuration if the random walk is transient, and a recurrent configuration otherwise. Since $I$ is connected, the transience or recurrence of $S$ does not depend on the choice of the starting point $x$.

The corresponding electrical network arises as follows. For $x \in I$, we denote by $B_{n}(x)$ the set of all vertices $y$ of $I$ such that $\delta(x, y) \leq n$, and we write $\partial B_{n}(x)=$ $B_{n}(x) \backslash B_{n-1}(x)$. We turn $B_{n}(x)$ into a graph by adding all induced (open) edges of $I$. Next we turn this graph into an electrical network by replacing each edge by of $I$. Next we turn this graph into an electrical network by replacing each edge by
a unit resistor, and by 'shorting together' all vertices in $\partial B_{n}(x)$. Let $R_{n}(x)$ be the a unit resistor, and by 'shorting together' all vertices in $\partial B_{n}(x)$. Let $R_{n}(x)$ be
effective resistance of the network between $x$ and the composite vertex $\partial B_{n}(x)$.

By an argument using monotonicity of effective resistance (as a function of the
By anstance of the network between $x$ and the composite vertex $\partial B_{n}(x)$. individual resistances), the increasing limit $R_{\infty}(x)=\lim _{n \rightarrow \infty} R_{n}(x)$ exists for all $x$ It is a consequence of the relationship between random walk and electrical networks that the random walk on $I$, beginning at $x$, is transient if and only if $R_{\infty}(x)<\infty$. Therefore Theorem 10.1 is a consequence of the following.

Theorem 10.2. Let $p>p_{\mathrm{c}}$, and let $I$ be the (a.s.) unique infinite open cluster.

(a) If $d=2$ then $R_{\infty}(x)=\infty$ for all $x \in I$.

(b) If $d \geq 3$ then $P_{p}\left(R_{\infty}(0)<\infty \mid 0 \in I\right)=1$.

Part (a) is obvious, as follows. The electrical resistance of a graph can only ncrease if any individual edge-resistance is increased. Since the network on $I$ may be obtained from that on $\mathbb{L}^{2}$ by setting the resistances of closed edges to $\infty$, we have that $R_{\infty}(x)$ is no smaller than the resistance 'between $x$ and $\infty$ ' of $\mathbb{L}^{2}$. The

${ }^{6}$ For a quite different and more recent approach, see [54]. 

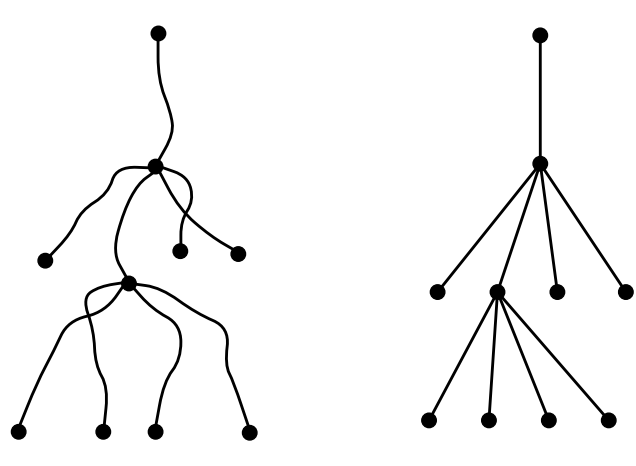

Fig. 10.1. The left picture depicts a tree-like subgraph of the lattice. The right picture is obtained by nent paths by single edges. The resistance of such an edge emanating from the $k$ th generation has order $\beta^{k}$.

latter resistance is infinite (since random walk is recurrent, or by direct estimation), implying that $R_{\infty}(x)=\infty$.

Part (b) is harder, and may be proved by showing that $I$ contains a subgraph having finite resistance. We begin with a sketch of the proof. Consider first a tree $T$ of 'down-degree' 4; see Figure 10.1. Assume that any edge joining the $k$ th generation to the $(k+1)$ th generation has electrical resistance $\beta^{k}$ where $\beta>1$. Using the series and parallel laws, the resistance of the tree, between the root and infinity, is $\sum_{k}(\beta / 4)^{k}$; this is finite if $\beta<4$. Now we do a little geometry. Let us try to imbed such a tree in the lattice $\mathbb{L}^{3}$, in such a way that the vertices of the tree are vertices of the lattice, and that the edges of the tree are paths of the lattice which are 'almost' disjoint. Since the resistance from the root to a point in the $k$ th generation is

$$
\sum_{r=0}^{k-1} \beta^{r}=\frac{\beta^{k}-1}{\beta-1}
$$

it is reasonable to try to position the $k$ th generation vertices on or near the surface $\partial B\left(\beta^{k-1}\right)$. The number of $k$ th generation vertices if $4^{k}$, and the volume of $\partial B\left(\beta^{k-1}\right)$ has order $\beta^{(k-1)(d-1)}$. The above construction can therefore only succeed when $4^{k}<\beta^{(k-1)(d-1)}$ for all large $k$, which is to say that $\beta>4^{1 /(d-1)}$

This crude picture suggests the necessary inequalities

$$
4^{1 /(d-1)}<\beta<4,
$$

which can be satisfied if and only if $d \geq 3$.

Assume now that $d \geq 3$. Our target is to show that the infinite cluster $I$ contains sufficiently many disjoint paths to enable a comparison of its effective resistance with that of the tree in Figure 10.1, and with some value of $\beta$ satisfying (10.3). In presenting a full proof of this, we shall use the following two percolation estimates, which are consequences of the results of Chapter 7.
Lemma 10.4. Assume that $p>p_{\mathrm{c}}$.

(a) There exists a strictly positive constant $\gamma=\gamma(p)$ such that

$$
P_{p}(B(n) \leftrightarrow \infty) \geq 1-e^{-\gamma n} \quad \text { for all } n .
$$

(b) Let $\sigma>1$, and let $A(n, \sigma)$ be the event that there exist two vertices inside $B(n)$ with the property that each is joined by an open path to $\partial B(\sigma n)$ but that there is no open path of $B(\sigma n)$ joining these two vertices. There exists a strictly positive constant $\delta=\delta(p)$ such that

$$
P_{p}(A(n, \sigma)) \leq e^{-\delta n(\sigma-1)} \quad \text { for all } n .
$$

We restrict ourselves here to the case $d=3$; the general case $d \geq 3$ is similar. The surface of $B(n)$ is the union of six faces, and we concentrate on the face

$$
F(n)=\left\{x \in \mathbb{Z}^{3}: x_{1}=n,\left|x_{2}\right|,\left|x_{3}\right| \leq n\right\} .
$$

We write $B_{k}=B\left(3^{k}\right)$ and $F_{k}=F\left(3^{k}\right)$. On $F_{k}$, we distinguish $4^{k}$ points, namely

$$
x_{k}(i, j)=\left(i d_{k}, j d_{k}\right), \quad-2^{k-1}<i, j \leq 2^{k-1}
$$

where $d_{k}=\left\lfloor(4 / 3)^{k}\right\rfloor$. The $x_{k}(i, j)$ are distributed on $F_{k}$ in the manner of a rectangular grid, and they form the 'centres of attraction' corresponding to the $k$ th generation of the tree discussed above.

With each $x_{k}(i, j)$ we associate four points on $F_{k+1}$, namely those in the set

$$
I_{k}(i, j)=\left\{x_{k+1}(r, s): r=2 i-1,2 i, s=2 j-1,2 j\right\} .
$$

These four points are called children of $x_{k}(i, j)$. The centroid of $I_{k}(i, j)$ is denoted $\bar{I}_{k}(i, j)$. We shall attempt to construct open paths from points near $x_{k}(i, j)$ to points near to each member of $I_{k}(i, j)$, and this will be achieved with high probability. In order to control the geometry of such paths, we shall build them within certain 'tubes' to be defined next.

Write $L(u, v)$ for the set of vertices lying within euclidean distance $\sqrt{3}$ of the line segment of $\mathbb{R}^{3}$ joining $u$ to $v$. Let $a>0$. Define the region

$$
T_{k}(i, j)=A_{k}(i, j) \cup C_{k}(i, j)
$$

where

$$
\begin{aligned}
& A_{k}(i, j)=B(a k)+L\left(x_{k}(i, j), \bar{I}_{k}(i, j)\right) \\
& C_{k}(i, j)=B(a k)+\bigcup_{x \in I_{k}(i, j)} L\left(\bar{I}_{k}(i, j), x\right) .
\end{aligned}
$$




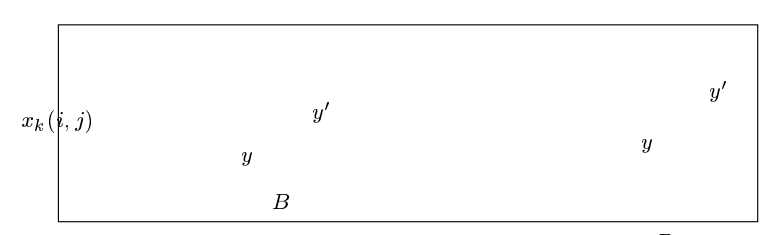

$B$

Fig. 10.2. A diagram of the region $T_{k}(i, j, j)$, with the points $Y_{k}(i, j)$ marked. The larger box is an enlargement of the box $B$ on the left. In the larger box appear open paths of the sort required for the corresponding event $E_{u}$, where $y=y_{u}$. Note that
the two smaller boxes within $B$ are joined to the surface of $B$, and that any two such connections are joined to one another within $B$.

See Figure 10.2

Within each $T_{k}(i, j)$ we construct a set of vertices as follows. In $A_{k}(i, j)$ we find vertices $y_{1}, y_{2}, \ldots, y_{t}$ such that the following holds. Firstly, there exists a constant $\nu$ such that $t \leq \nu 3^{k}$ for all $k$. Secondly, each $y_{u}$ lies in $A_{k}(i, j)$

$$
y_{u} \in L\left(x_{k}(i, j), \bar{I}_{k}(i, j)\right), \quad \frac{1}{3} a k \leq \delta\left(y_{u}, y_{u+1}\right) \leq \frac{2}{3} a k
$$

for $1 \leq u<t$, and furthermore $y_{1}=x_{k}(i, j)$, and $\left|y_{t}-\bar{I}_{k}(i, j)\right| \leq 1$.

Likewise, for each $x \in I_{k}(i, j)$, we find a similar sequence $y_{1}(x), y_{2}(x), \ldots, y_{v}(x)$ satisfying (10.7) with $x_{k}(i, j)$ replaced by $x$, and with $y_{1}(x)=y_{t}, y_{v}(x)=x$, and $v=v(x) \leq \nu 3^{k}$.

The set of all such $y$ given above is denoted $Y_{k}(i, j)$. We now construct open paths using $Y_{k}(i, j)$ as a form of skeleton. Let $0<7 b<a$. For $1 \leq u<t$, let $E_{u}=E_{u}(k, i, j)$ be the event that

(a) there exist $z_{1} \in y_{u}+B(b k)$ and $z_{2} \in y_{u+1}+B(b k)$ such that $z_{i} \leftrightarrow y_{u}+\partial B(a k)$ for $i=1,2$, and

(b) any two points lying in $\left\{y_{u}, y_{u+1}\right\}+B(b k)$ which are joined to $y_{u}+\partial B(a k)$ are also joined to one another within $y_{u}+\partial B(a k)$

We define similar events $E_{x, u}=E_{x, u}(k, i, j)$ for $x \in I_{k}(i, j)$ and $1 \leq u<v=v(x)$, and finally let

$$
\mathcal{E}_{k}(i, j)=\left\{\bigcap_{1 \leq u<t} E_{u}\right\} \cap\left\{\bigcap_{\substack{1 \leq u<v(x) \\ x \in I_{k}(i, j)}} E_{x, u}\right\} .
$$

Let us estimate $P_{p}\left(\mathcal{E}_{k}(i, j)\right)$. Using Lemma 10.4 , we have that

$$
P_{p}\left(\overline{\mathcal{E}_{k}(i, j)}\right) \leq 5 \nu 3^{k} e^{-\gamma b k}+5 \nu 3^{k} e^{-\delta a k / 6} .
$$

We call $x_{m+l}(r, s)$ a descendant of $x_{m}(0,0)$ if it is a child of a child $\ldots$ of a child of $x_{m}(0,0)$. Write $\mathcal{K}_{m}$ for the set of all $(m+l, r, s)$ such that $x_{m+l}(r, s)$ is a descendant of $x_{m}(0,0)$. We have from $(10.8)$ that

$$
U_{m}=\sum_{(k, r, s) \in \mathcal{K}_{m}} P_{p}\left(\overline{\mathcal{E}_{k}(r, s)}\right) \leq \sum_{k=m}^{\infty} 4^{k-m} 5 \nu 3^{k}\left(e^{-\gamma b k}+e^{-\delta a k / 6}\right)
$$

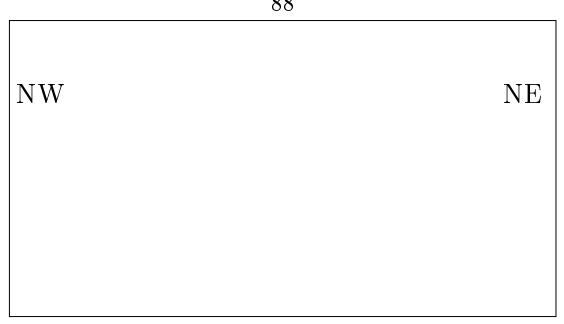

Fig. 10.3. NW and NE reflectors in action

Now pick $a, b$ such that $0<7 b<a$ and $e^{-\gamma b}, e^{-\delta a / 6}<\frac{1}{12}$, so that $U_{m} \rightarrow 0$ as $m \rightarrow \infty$. This implies that there exists a (random) value $M$ of $m$ such that $\mathcal{E}_{k}(r, s)$ occurs for all $(k, r, s) \in \mathcal{K}_{M}$

Turning to the geometry implied in the definition of the $\mathcal{E}_{k}(r, s)$, we find that the infinite open cluster contains a topological copy of the tree in Figure 10.1, where the length of a path joining a $k$ th generation vertex to one of its children is no greater than $C k^{3} 3^{k}$ for some constant $C$. In particular, this length is smaller than $C^{\prime} \beta^{k}$ for any $\beta$ satisfying $3<\beta<4$ and for some $C^{\prime}=C^{\prime}(\beta)$. Choosing $\beta$ and $C^{\prime}$ accordingly, and referring to the discussion around (10.3), we conclude that contains a tree having finite resistance between its root and infinity. The second claim of Theorem 10.2 follows.

\subsection{Random Walks in Two-Dimensional Labyrinths}

A beautiful question dating back to Lorentz [245] and Ehrenfest [129] concerns the behaviour of a particle moving in $\mathbb{R}^{d}$ but scattered according to reflecting obstacles distributed about $\mathbb{R}^{d}$. There is a notorious lattice version of this question which is largely unsolved. Start with the two-dimensional square lattice $\mathbb{L}^{2}$. A reflector may be placed at any vertex in either of two ways: either it is a NW reflector (which deflects incoming rays heading northwards, resp. southwards, to the west, resp. east, and vice versa) or it is a NE reflector (defined similarly); see Figure 10.3. Think of a reflector as being a two-sided mirror placed at $45^{\circ}$ to the axes, so that an incoming light ray is reflected along an axis perpendicular to its direction of arrival. Now, for each vertex $x$, with probability $p$ we place a reflector at $x$, and otherwise we place nothing at $x$. This is done independently for different $x$. If a reflector is placed at $x$, then we specify that it is equally likely to be $\mathrm{NW}$ as NE.

We shine a torch northwards from the origin. The light is reflected by the mirrors, and we ask whether or not the light ray returns to the origin. Letting

$$
\eta(p)=P_{p} \text { (the light ray returns to the origin) }
$$

we would like to know for which values of $p$ it is the case that $\eta(p)=1$. It is reasonable to conjecture that $\eta$ is non-decreasing in $p$. Certainly $\eta(0)=0$, and it is well known' that $\eta(1)=1$. 

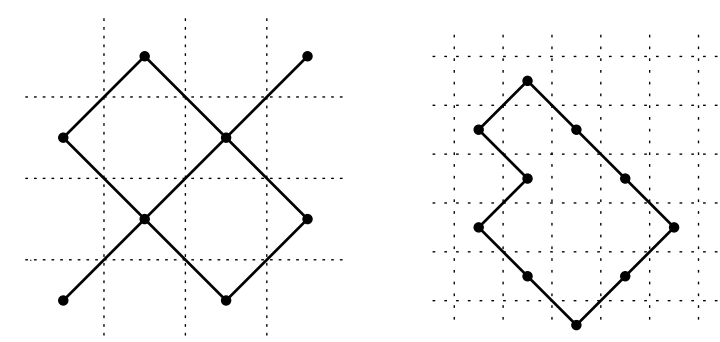

Fig. 10.4. (a) The heavy lines form the lattice $\mathbb{L}_{A}^{2}$, and the central point is the origin of $\mathbb{L}^{2}$. (b) An open circuit in $\mathbb{L}_{A}^{2}$ constitutes a barrier of mirrors through which no light may penetrate.

Theorem 10.9. It is the case the $\eta(1)=1$.

Proof of Theorem 10.9. This proof is alluded to in [G] and included in [82]. From $\mathbb{L}^{2}$ we construct an ancillary lattice $\mathbb{L}_{A}^{2}$ as follows. Let

$$
A=\left\{\left(m+\frac{1}{2}, n+\frac{1}{2}\right): m+n \text { is even }\right\} .
$$

On $A$ we define the adjacency relation $\sim$ by $\left(m+\frac{1}{2}, n+\frac{1}{2}\right) \sim\left(r+\frac{1}{2}, s+\frac{1}{2}\right)$ if and only if $|m-r|=|n-s|=1$, obtaining thereby a copy of $\mathbb{L}^{2}$ denoted as $\mathbb{L}_{A}^{2}$. See Figure 10.4 .

We now use the above 'labyrinth' to define a bond percolation process on $\mathbb{L}_{A}^{2}$. We declare the edge of $\mathbb{L}_{A}^{2}$ joining $\left(m-\frac{1}{2}, n-\frac{1}{2}\right)$ to $\left(m+\frac{1}{2}, n+\frac{1}{2}\right)$ to be open if there is a NE mirror at $(m, n)$; similarly we declare the edge joining $\left(m-\frac{1}{2}, n+\frac{1}{2}\right)$ to $\left(m+\frac{1}{2}, n-\frac{1}{2}\right)$ to be open if there is a NW mirror at $(m, n)$. Edges which are not open are designated closed. This defines a percolation model in which northeasterly edges (resp. north-westerly edges) are open with probability $p_{\mathrm{NE}}=\frac{1}{2}$ (resp. $p_{\mathrm{NW}}=\frac{1}{2}$ ). Note that $p_{\mathrm{NE}}+p_{\mathrm{NW}}=1$, which implies that the percolation model is critical (see [G, 202]).

Let $N$ be the number of open circuits in $\mathbb{L}_{A}^{2}$ which contain the origin in their interiors. Using general results from percolation theory, we have that $\mathbb{P}(N \geq 1)=1$, where $\mathbb{P}$ is the appropriate probability measure. (This follows from the fact that $\theta\left(\frac{1}{2}\right)=0 ;$ cf. Theorem 9.1, see also [G, 181, 202].) However, such an open circuit corresponds to a barrier of mirrors surrounding the origin, from which no light can escape (see Figure 10.4 again). Therefore $\eta(1)=1$.

We note that the above proof is valid in the slightly more general setting in which $\mathrm{NE}$ mirrors are present with density $p_{\mathrm{NE}}$ and NW mirrors with density $p_{\mathrm{NW}}$ where $p_{\mathrm{NW}}+p_{\mathrm{NE}}=1$ and $0<p_{\mathrm{NW}}<1$. This generalisation was noted in [82].

When $0<p<1$, the question of whether or not $\eta(p)=1$ is wide open, despite many attempts to answer it? ${ }^{7}$. It has been conjectured that $\eta(p)=1$ for all $p>$

${ }^{7}$ I heard of this problem in a conversation with Hermann Rost and Frank Spitzer in Heidelberg in 1978 . The proof that $\eta(1)=1$ was known to me (and presumably to others) in 1978 also.
0 , based on numerical simulations; see [111, 376]. Some progress has been made recently by Quas [321].

The above lattice version of the 'mirror model' appears to have been formulated first around 20 years ago. In a systematic approach to random environments of reflectors, Ruijgrok and Cohen [325] proposed a programme of study of 'mirror' reflectors, Ruijgrok and Cohen [325] proposed a programme of study of 'mirror'
and 'rotator' models. Since then, there have been reports of many Monte Carlo experiments, and several interesting conjectures have emerged (see $[109,110,111$, $344,376])$. Rigorous progress has been relatively slight; see $[82, \mathrm{G}, 321]$ for partial results.

The principal difficulty in the above model resides in the facts that the environment is random but that the trajectory of the light is (conditionally) deterministic. If we relax the latter determinism, then we arrive at model which is more tractable. In this new version, there are exactly three types of point, called mirrors, crossings, In this new version, there are exactly three types of point, called mirrors, crossings,
and random walk $(r w)$ points. Let $p_{\mathrm{rw}}, p_{+} \geq 0$ be such that $p_{\mathrm{rw}}+p_{+} \leq 1$. We and random walk $(r w)$ points,
designate each vertex $x$ to be

$$
\text { a random walk (rw) point, with probability } p_{\mathrm{rw}} \text {, }
$$

a crossing, with probability $p_{+}$,

a mirror, otherwise.

If a vertex is a mirror, then it is occupied by a NW reflector with probability $\frac{1}{2}$, and otherwise by a NE reflector. The environment of mirrors and rw points is denoted by $Z=\left(Z_{x}: x \in \mathbb{Z}^{2}\right)$ and is termed a 'labyrinth'; we write $\mathbb{P}$ for the probability measure associated with the labyrinth, so that $\mathbb{P}$ is product measure on the corresponding environment space.

The physical meaning of these terms is as follows. Suppose that some vertex $x$ is occupied by a candle, which emits light rays along the four axes leaving $x$. When a ray is incident with a mirror, then it is reflected accordingly. When a ray encounters a crossing, then it continues undeflected. When a ray encounters a rw point, then it leaves this point in one of the four available directions, chosen at random in the manner of a random walk.

We formalise this physical explanation by defining a type of random walk $X=$ $\left(X_{0}, X_{1}, \ldots\right)$ on $\mathbb{L}^{2}$. Assume that $p_{\mathrm{rw}}>0$, and sample a random labyrinth $Z$ according to the measure $\mathbb{P}$. Let $x$ be a rw point, and set $X_{0}=x$. We choose a random neighbour $X_{1}$ of $x$, each of the four possibilities being equally likely. Having constructed $X_{0}, X_{1}, \ldots, X_{r}$, we define $X_{r+1}$ as follows. If $X_{r}$ is a rw point, we let $X_{r+1}$ be a randomly chosen neighbour of $X_{r}$ (chosen independently of all earlier choices); if $X_{r}$ is not a rw point, then we define $X_{r+1}$ to be the next vertex illuminated by a ray of light which is incident with $X_{r}$ travelling in the direction $X_{r}-X_{r-1}$. The consequent sequence $X$ is called a 'random walk in a random labyrinth'. Let $P_{x}^{Z}$ denote the law of $X$, conditional on $Z$, and starting at $x$. We say that the rw point $x$ is $Z$-recurrent if there exists $\left(P_{r}^{Z}\right.$-a.s.) an integer $N$ such that $X_{N}=x$, and otherwise we say that $x$ is $Z$-transient. We say that the labyrinth $Z$ is recurrent if every rw point is $Z$-recurrent. It is easily seen, using the translationinvariance of $\mathbb{P}$ and the zero-one law, that the labyrinth is $\mathbb{P}$-a.s. recurrent if and only if

$\mathbb{P}(0$ is $Z$-recurrent $\mid 0$ is a rw point $)=1$. 


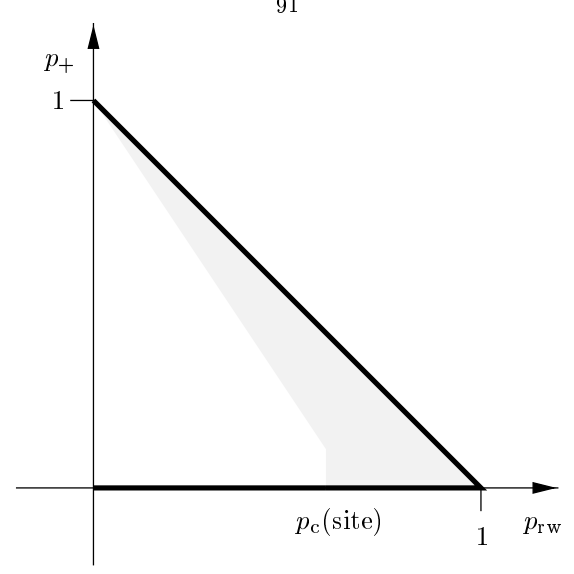

Fig. 10.5. The grey region and the heavy lines of the figure indicate the part of $\left(p_{\mathrm{rw}}, p_{+}\right)$space for which non-localisation is proved. The labyrinth is a.s. localised

Theorem 10.10. If $p_{\mathrm{rw}}>0$ then the labyrinth $Z$ is $\mathbb{P}$-a.s. recurrent.

This theorem, together with most other results in this chapter, appears in [166], and is proved by showing that a corresponding electrical network has infinite resistance. A brief proof appears at the end of this section.

Remembering that irreducible Markov chains on finite state spaces are necessarily recurrent, we turn our attention to a question of 'localisation'. Let $x$ be a rw point in the random labyrinth $Z$, and let $X$ be constructed as above. We say that $x$ is $Z$-localised if $X$ visits $\left(P_{x}^{Z}\right.$-a.s. $)$ only finitely many vertices; we call $x$ $Z$-non-localised otherwise. We say that the random labyrinth $Z$ is localised if all rw points are $Z$-localised, and we call it non-localised otherwise. Using the translationinvariance of $Z$ and the zero-one law, we may see that $Z$ is $\mathbb{P}$-a.s. localised if and only if

$$
\mathbb{P}(0 \text { is } Z \text {-localised } \mid 0 \text { is a rw point })=1 .
$$

Theorem 10.11. Let $p_{\mathrm{rw}}>0$. There exists a strictly positive constant $A=A\left(p_{\mathrm{rw}}\right)$ such that the following holds. The labyrinth $Z$ is $\mathbb{P}$-a.s. non-localised if any of the following conditions hold:

(a) $p_{\mathrm{rw}}>p_{\mathrm{c}}\left(\right.$ site), the critical probability of site percolation on $\mathbb{L}^{2}$,

(b) $p_{+}=0$,

(c) $p_{\text {rw }}+p_{+}>1-A$.

We shall see in the proof of part (c) (see Theorem 10.17) that $A\left(p_{\mathrm{rw}}\right) \rightarrow 0$ as $p_{\text {rw }} \downarrow 0$. This fact is reported in Figure 10.5, thereby correcting an error in the corresponding figure contained in [166].

Proof of Theorem 10.10. Assume $p_{\text {rw }}>0$. We shall compare the labyrinth with a certain electrical network. By showing that the effective resistance of this network between 0 and $\infty$ is a.s. infinite, we shall deduce that $Z$ is a.s. recurrent. For details of the relationship between Markov chains and electrical networks, see the book [118] and the papers [251, 277].

By the term $Z$-path we mean a path of the lattice (possibly with self-intersections) which may be followed by the light: i.e., at rw points it is unconstrained, while at reflectors and crossings it conforms to the appropriate rule. A formal definition will be presented in Section 10.3 .

Let $e=\langle u, v\rangle$ be an edge of $\mathbb{L}^{2}$. We call $e$ a normal edge if it lies in some $Z$-path $\pi(e)$ which is minimal with respect to the property that its two endvertices (and no others) are rw points, and furthermore that these two endvertices are distinct. If $e$ is normal, we write $l(e)$ for the number of edges in $\pi(e)$; if $e$ is not normal, we write $l(e)=0$. We define $\rho(e)=1 / l(e)$, with the convention that $1 / 0=\infty$.

Next, we construct an electrical network $E(\rho)$ on $\mathbb{L}^{2}$ by, for each edge $e$ of $\mathbb{L}^{2}$, placing an electrical resistor of size $\rho(e)$ at $e$. Let $R=R(Z)$ be the effective resistance of this network between 0 and $\infty$ (which is to say that $R=\lim _{n \rightarrow \infty} R_{n}$, where $R_{n}$ is the resistance between 0 and a composite vertex obtained by identifying all vertices in $\partial B(n))$.

Lemma 10.12. We have that $\mathbb{P}(R(Z)=\infty \mid 0$ is a rw point $)=1$.

Proof. We define the 'edge-boundary' $\Delta_{\mathrm{e}} B(n)$ of $B(n)$ to be the set of edges $e=$ $\langle x, y\rangle$ with $x \in \partial B(n)$ and $y \in \partial B(n+1)$. We claim that there exists a positive constant $c$ and a random integer $M$ such that

(10.13) $\quad \rho(e) \geq \frac{c}{\log n}$ for all $e \in \Delta_{\mathrm{e}} B(n)$ and $\quad n \geq M$.

To show this, we argue as follows. Assume that $e=\langle x, y\rangle$ is normal, and let $\lambda_{1}$ be the number of edges in the path $\pi(e)$ on one side of $e$ (this side being chosen in an arbitrary way), and $\lambda_{2}$ for the number on the other side. Since each new vertex an arbitrary way), and $\lambda_{2}$ for the number on the other side. Since each new vertex
visited by the path is a rw point with probability $p_{\text {rw }}$, and since no vertex appears more than twice in $\pi(e)$, we have that

$\mathbb{P}(l(e)>2 k, e$ is normal $) \leq 2 \mathbb{P}\left(\lambda_{1} \geq k, e\right.$ is normal $) \leq 2\left(1-p_{\text {rw }}\right)^{\frac{1}{2}(k-1)}$.

Therefore, for $c>0$ and all $n \geq 2$,

$\mathbb{P}\left(\rho(e)<\frac{c}{\log n}\right.$ for some $\left.e \in \Delta_{\mathrm{e}} B(n)\right) \leq 4(2 n+1) \mathbb{P}\left(l(e)>\frac{\log n}{c}, e\right.$ is normal $)$ $\leq \beta n^{1-\alpha}$

where $\alpha=\alpha(c)=-(4 c)^{-1} \log \left(1-p_{\mathrm{rw}}\right)$ and $\beta=\beta\left(c, p_{\mathrm{rw}}\right)<\infty$. We choose $c$ such that $\alpha>\frac{5}{2}$, whence (10.13) follows by the Borel-Cantelli lemma.

The conclusion of the lemma is a fairly immediate consequence of (10.13), using the usual argument which follows. From the electrical network $E(\rho)$ we construct another network with no larger resistance. This we do by identifying all vertices contained in each $\partial B(n)$. In this new system there are $\left|\Delta_{\mathrm{e}} B(n)\right|$ parallel connections 
between $\partial B(n)$ and $\partial B(n+1)$, each of which has (for $n \geq M)$ a resistance at least $c / \log n$. The effective resistance from the origin to infinity is therefore at least

$$
\sum_{n=M}^{\infty} \frac{c}{\left|\Delta_{\mathrm{e}} B(n)\right| \log n}=\sum_{n=M}^{\infty} \frac{c}{4(2 n+1) \log n}=\infty,
$$

and the proof of the lemma is complete.

Returning to the proof of Theorem 10.10, suppose that 0 is a rw point, and consider a random walk $X$ with $X_{0}=0$. Let $C_{0}$ be the set of rw points which may be reached by light originating at $0 ; C_{0}$ is the state space of the embedded Markov chain obtained by sampling $X$ at times when it visits rw points. This embedded chain constitutes an irreducible time-reversible Markov chain on $C_{0}$. There is a corresponding electrical network with nodes $C_{0}$, and with resistors of unit resistance joining every distinct pair $u, v$ of such sites which are joined by some $Z$-path which visits no rw point other than its endpoints. This may be achieved by assigning to each corresponding edge $e$ of $\mathbb{L}^{2}$ the resistance $\rho(e)$. Since the latter network may be obtained from $E(\rho)$ by deleting certain connections between paths, we have tha the embedded Markov chain on $C_{0}$ is recurrent if $E(\rho)$ has infinite resistance. This latter fact was proved in Lemma 10.12 .

Proof of Theorem 10.11. Part (c) will be proved in the next section, as part of Theorem 10.17. We begin with part (a). If $p_{\text {rw }}>p_{\mathrm{c}}(\mathrm{site})$, then there exists a.s. a unique infinite cluster $I$ of rw points having strictly positive density. Suppose $x \in I$. The walk $X$ will $\left(P_{x}^{Z}\right.$-a.s. $)$ visit every vertex in $I$, whence the labyrinth is non-localised.

Next we prove (b), of which the proof is similar to that of Theorem 10.9. This time we construct two copies of $\mathbb{L}^{2}$ as follows. Let

$$
A=\left\{\left(m+\frac{1}{2}, n+\frac{1}{2}\right): m+n \text { is even }\right\}, B=\left\{\left(m+\frac{1}{2}, n+\frac{1}{2}\right): m+n \text { is odd }\right\} .
$$

On $A \cup B$ we define the adjacency relation $\left(m+\frac{1}{2}, n+\frac{1}{2}\right) \sim\left(r+\frac{1}{2}, s+\frac{1}{2}\right)$ if and only if $|m-r|=1$ and $|n-s|=1$, obtaining thereby two copies of $\mathbb{L}^{2}$ denoted respectively as $\mathbb{L}_{A}^{2}$ and $\mathbb{L}_{B}^{2}$. See Figure 10.6 .

We now define bond percolation processes on $\mathbb{L}_{A}^{2}$ and $\mathbb{L}_{B}^{2}$. Assume $p_{+}=0$. We present the rules for $\mathbb{L}_{A}^{2}$ only; the rules for $\mathbb{L}_{B}^{2}$ are analogous. An edge of $\mathbb{L}_{A}^{2}$ joining $\left(m-\frac{1}{2}, n-\frac{1}{2}\right)$ to $\left(m+\frac{1}{2}, n+\frac{1}{2}\right)$ to declared to be open if there is a NE mirro at $(m, n)$; similarly we declare the edge joining $\left(m-\frac{1}{2}, n+\frac{1}{2}\right)$ to $\left(m+\frac{1}{2}, n-\frac{1}{2}\right)$ to be open if there is a NW mirror at $(m, n)$. Edges which are not open are called closed. This defines percolation models on $\mathbb{L}_{A}^{2}$ and $\mathbb{L}_{B}^{2}$ in which north-easterly edges (resp. north-westerly edges) are open with probability $p_{\mathrm{NE}}=\frac{1}{2}\left(1-p_{\mathrm{rw}}\right)$ (resp. $\left.p_{\mathrm{NW}}=\frac{1}{2}\left(1-p_{\mathrm{rw}}\right)\right)$. These processes are subcritical since $p_{\mathrm{NE}}+p_{\mathrm{NW}}=1-p_{\mathrm{rw}}<1$ Therefore, there exists ( $\mathbb{P}$-a.s.) no infinite open path in either $\mathbb{L}_{A}^{2}$ or $\mathbb{L}_{B}^{2}$, and we assume henceforth that no such infinite open path exists.

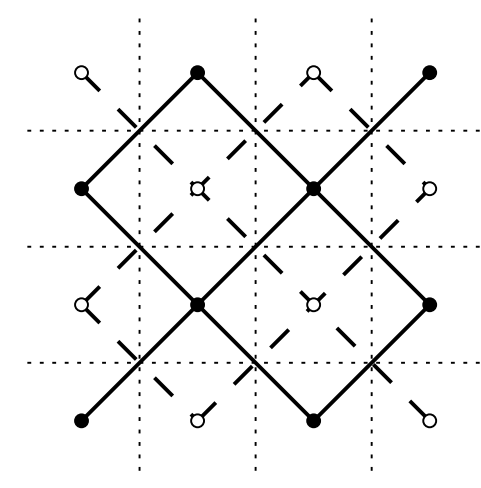

Fig. 10.6. The heavy lines are the edges of the lattice $\mathbb{L}_{A}^{2}$, and the dashed lines are the edges of the lattice $\mathbb{L}_{B}^{2}$

Let $N(A)($ resp. $N(B))$ be the number of open circuits in $\mathbb{L}_{A}^{2}$ (resp. $\mathbb{L}_{B}^{2}$ ) which contain the origin in their interiors. Since the above percolation processes are subcritical, there exists (by Theorem 6.10) a strictly positive constant $\alpha=\alpha\left(p_{\mathrm{NW}}, p_{\mathrm{NE}}\right)$ such that

(10.14)

$\mathbb{P}\left(x\right.$ lies in an open cluster of $\mathbb{L}_{A}^{2}$ of diameter at least $\left.n\right) \leq e^{-\alpha n}$ for all $n$,

for any vertex $x$ of $\mathbb{L}_{A}^{2}$. (By the diameter of a set $C$ of vertices, we mean $\max \{|y-z|$ : $y, z \in C\}$.) The same conclusion is valid for $\mathbb{L}_{B}^{2}$. We claim that

$$
\mathbb{P}(0 \text { is a rw point, and } N(A)=N(B)=0)>0,
$$

and we prove this as follows. Let $\Lambda(k)=[-k, k]^{2}$, and let $N_{k}(A)$ (resp. $\left.N_{k}(B)\right)$ be the number of circuits contributing to $N(A)$ (resp. $N(B)$ ) which contain only points lying strictly outside $\Lambda(k)$. If $N_{k}(A) \geq 1$ then there exists some vertex $\left(m+\frac{1}{2}, \frac{1}{2}\right)$ of lying strictly outside $\Lambda(k)$. If $N_{k}(A) \geq 1$ then there exists some vertex $\left(m+\frac{1}{2}, \frac{1}{2}\right)$ of
$\mathbb{L}^{2}$, with $m \geq k$, which belongs to an open circuit of diameter exceeding $m$. Using $\mathbb{L}_{A}^{2}$, with
$(10.14)$,

$$
\mathbb{P}\left(N_{k}(A) \geq 1\right) \leq \sum_{m=k}^{\infty} e^{-\alpha m}<\frac{1}{3}
$$

for sufficiently large $k$. We pick $k$ accordingly, whence

$$
\mathbb{P}\left(N_{k}(A)+N_{k}(B) \geq 1\right) \leq \frac{2}{3} .
$$

Now, if $N_{k}(A)=N_{k}(B)=0$, and in addition all points of $\mathbb{L}^{2}$ inside $\Lambda(k)$ are rw points, then $N(A)=N(B)=0$. These last events have strictly positive probabilities, and (10.15) follows.

Let $J$ be the event that there exists a rw point $x=x(Z)$ which lies in the interior of no open circuit of either $\mathbb{L}_{A}^{2}$ or $\mathbb{L}_{B}^{2}$. Since $J$ is invariant with respect to 

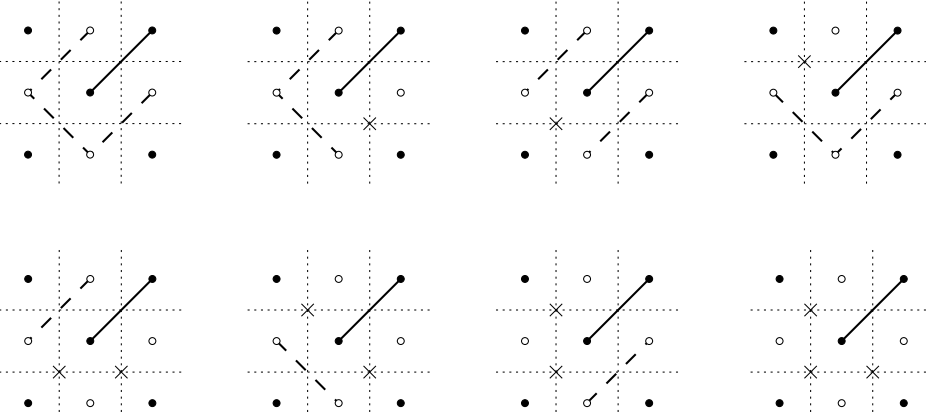

Fig. 10.7. The solid line in each picture is the edge $e=\langle u, v\rangle$, and the central vertex is $u$. If all three of the other edges of $\mathbb{L}_{A}^{2}$ incident with the vertex $u$ are closed in $\mathbb{L}_{A}^{2}$ then there are eight possibilities for the corresponding edges of $\mathbb{L}_{B}^{2}$. The dashed lines indicate open edges of $\mathbb{L}_{B}^{2}$, and the crosses mark rw points of $\mathbb{L}^{2}$. In every picture,

light incident with one side of the mirror at $e$ will illuminate the other side also.

translations of $\mathbb{L}^{2}$, and since $\mathbb{P}$ is product measure, we have that $\mathbb{P}(J)$ equals either 0 or 1 . Using (10.15), we deduce that $\mathbb{P}(J)=1$. Therefore we may find a.s. some such vertex $x=x(Z)$. We claim that $x$ is $Z$-non-localised, which will imply as claimed that the labyrinth if a.s. non-localised.

Let $C_{x}$ be the set of rw points reachable by light originating at the rw point $x$. The set $C_{x}$ may be generated in the following way. We allow light to leave $x$ along the four axial directions. When a light ray hits a crossing or a mirror, it follows the associated rule; when a ray hits a rw point, it causes light to depart the point along each of the other three axial directions. Now $C_{x}$ is the set of rw points thus reached. Following this physical picture, let $F$ be the set of 'frontier mirrors', i.e., the set of mirrors only one side of which is illuminated. Assume that $F$ is non-empty, say $F$ contains a mirror at some point $(m, n)$. Now this mirror must correspond to an open edge $e$ in either $\mathbb{L}_{A}^{2}$ and $\mathbb{L}_{B}^{2}$ (see Figure 10.6 again), and we may assume without loss of generality that this open edge $e$ is in $\mathbb{L}_{A}^{2}$. We write $e=\langle u, v\rangle$ where $u, v \in A$, and we assume that $v=u+(1,1)$; an exactly similar argument holds otherwise. There are exactly three other edges of $\mathbb{L}_{A}^{2}$ which are incident to $u$ (resp. $v$ ), and we claim that one of these is open. To see this, argue as follows. If none is open, then $u+\left(-\frac{1}{2}, \frac{1}{2}\right)$ either is a rw point or has a NE mirror,

$u+\left(-\frac{1}{2},-\frac{1}{2}\right)$ either is a rw point or has a NW mirror

$u+\left(\frac{1}{2},-\frac{1}{2}\right)$ either is a rw point or has a NE mirror.

See Figure 10.7 for a diagram of the eight possible combinations. By inspection, each such combination contradicts the fact that $e=\langle u, v\rangle$ corresponds to a frontier mirror.

Therefore, $u$ is incident to some other open edge $f$ of $\mathbb{L}_{A}^{2}$, other than $e$. By a further consideration of each of $2^{3}-1$ possibilities, we may deduce that there exists such an edge $f$ lying in $F$. Iterating the argument, we find that $e$ lies in eithe an open circuit or an infinite open path of $F$ lying in $\mathbb{L}_{A}^{2}$. Since there exists (by assumption) no infinite open path, this proves that $f$ lies in an open circuit of $F$ in $\mathbb{L}_{A}^{2}$.

By taking the union over all $e \in F$, we obtain that $F$ is a union of open circuits of $\mathbb{L}_{A}^{2}$ and $\mathbb{L}_{B}^{2}$. Each such circuit has an interior and an exterior, and $x$ lies (by assumption, above) in every exterior. There are various ways of deducing that $x$ is $Z$-non-localised, and here is such a way.

Assume that $x$ is $Z$-localised. Amongst the set of vertices $\{x+(n, 0): n \geq 1\}$, let $y$ be the rightmost vertex at which there lies a frontier mirror. By the above argument, $y$ belongs to some open circuit $G$ of $F$ (belonging to either $\mathbb{L}_{A}^{2}$ or $\mathbb{L}_{B}^{2}$ ), whose exterior contains $x$. Since $y$ is rightmost, we have that $y^{\prime}=y+(-1,0)$ is illuminated by light originating at $x$, and that light traverses the edge $\left\langle y^{\prime}, y\right\rangle$. Similarly, light does not traverse the edge $\left\langle y, y^{\prime \prime}\right\rangle$, where $y^{\prime \prime}=y+(1,0)$. Therefore, the point $y+\left(\frac{1}{2}, 0\right)$ of $\mathbb{R}^{2}$ lies in the interior of $G$, which contradicts the fact that $y$ is rightmost. This completes the proof for part (b).

\subsection{General Labyrinths}

There are many possible types of reflector, especially in three and more dimensions. Consider $\mathbb{Z}^{d}$ where $d \geq 2$. Let $I=\left\{u_{1}, u_{2}, \ldots, u_{d}\right\}$ be the set of positive unit vectors, and let $I^{ \pm}=\{-1,+1\} \times I$; members of $I^{ \pm}$are written as $\pm u_{j}$. We make the following definition. A reflector is a map $\rho: I^{ \pm} \rightarrow I^{ \pm}$satisfying $\rho(-\rho(u))=-u$ for all $u \in I^{ \pm}$. We denote by $\mathcal{R}$ the set of reflectors. The 'identity reflector' is called a crossing (this is the identity map on $I^{ \pm}$), and denoted by + .

The physical interpretation of a reflector is as follows. If light impinges on a reflector $\rho$, moving in a direction $u\left(\in I^{ \pm}\right)$then it is required to depart the reflector in the direction $\rho(u)$. The condition $\rho(-\rho(u))=-u$ arises from the reversibility of reflections.

Using elementary combinatorics, one may calculate that the number of distinct reflectors in $d$ dimensions is

$$
\sum_{s=0}^{d} \frac{(2 d) !}{(2 s) ! 2^{d-s}(d-s) !}
$$

A random labyrinth is constructed as follows. Let $p_{\text {rw }}$ and $p_{+}$be non-negative reals satisfying $p_{\mathrm{rw}}+p_{+} \leq 1$. Let $Z=\left(Z_{x}: x \in \mathbb{Z}^{d}\right)$ be independent random variables taking values in $\mathcal{R} \cup\{\varnothing\}$, with common mass function

$$
\mathbb{P}\left(Z_{0}=\alpha\right)= \begin{cases}p_{\text {rw }} & \text { if } \alpha=\varnothing \\ p_{+} & \text {if } \alpha=+ \\ \left(1-p_{\text {rw }}-p_{+}\right) \pi(\rho) & \text { if } \alpha=\rho \in \mathcal{R} \backslash\{+\},\end{cases}
$$

where $\pi$ is a prescribed probability mass function on $\mathcal{R} \backslash\{+\}$. We call a point $x$ a crossing if $Z_{x}=+$, and a random walk (rw) point if $Z_{x}=\varnothing$.

$\mathrm{A} \mathbb{L}^{d}$-path is defined to be a sequence $x_{0}, e_{0}, x_{1}, e_{1}, \ldots$ of alternating vertices $x_{i}$ and distinct edges $e_{j}$ such that $e_{j}=\left\langle x_{j}, x_{j+1}\right\rangle$ for all $j$. If the path has a final vertex $x_{n}$, then it is said to have length $n$ and to join $x_{0}$ to $x_{n}$. If it is infinite, then 
it is said to join $x_{0}$ to $\infty$. A $\mathbb{L}^{d}$-path may visit vertices more than once, but we insist that its edges be distinct.

We define a $Z$-path to be a $\mathbb{L}^{d}$-path $x_{0}, e_{0}, x_{1}, e_{1}, \ldots$ with the property that, for all $j$,

$$
x_{j+1}-x_{j}=Z_{x_{j}}\left(x_{j}-x_{j-1}\right) \quad \text { whenever } Z_{x_{j}} \neq \varnothing,
$$

which is to say that the path conforms to all reflectors.

Let $N$ be the set of rw points. We define an equivalence relation $\leftrightarrow$ on $N$ by $x \leftrightarrow y$ if and only if there exists a $Z$-path with endpoints $x$ and $y$. We denote by $C_{x}$ the equivalence class of $(N, \leftrightarrow)$ containing the rw point $x$, and by $\mathcal{C}$ the set of equivalence classes of $(N, \leftrightarrow)$. The following lemma will be useful; a sketch proof is deferred to the end of the section.

Lemma 10.16. Let $d \geq 2$ and $p_{\mathrm{rw}}>0$. The number $M$ of equivalence classes of $(N, \leftrightarrow)$ having infinite cardinality satisfies

$$
\text { either } \mathbb{P}(M=0)=1 \quad \text { or } \quad \mathbb{P}(M=1)=1 .
$$

Next we define a random walk in the random labyrinth $Z$. Let $x$ be a rw point. A walker, starting at $x$, flips a fair coin (in the manner of a symmetric random walker) whenever it arrives at a rw point in order to determine its next move. When it meets a reflector, it moves according to the reflector (i.e., if it strikes the reflector $\rho$ in the direction $u$, then it departs in the direction $\rho(u)$ ). Writing $P_{x}^{Z}$ for the law of the walk, we say that the point $x$ is $Z$-recurrent if $P_{x}^{Z}\left(X_{N}=x\right.$ for some $\left.N \geq 1\right)=1$, and $Z$-transient otherwise. As before, we say that $Z$ is transient if there exists a rw point $x$ which is $Z$-transient, and recurrent otherwise.

Note that, if the random walker starts at the rw point $x$, then the sequence of rw points visited constitutes an irreducible Markov chain on the equivalence class $C_{x}$. Therefore, the rw point $x$ is $Z$-localised if and only if $\left|C_{x}\right|<\infty$. As before, we say that $Z$ is localised if all rw points are $Z$-localised, and non-localised otherwise.

Theorem 10.17. Let $p_{\mathrm{rw}}>0$. There exists a strictly positive constant $A=$ $A\left(p_{\mathrm{rw}}, d\right)$ such that the following holds.

(a) Assume that $d \geq 2$. If $1-p_{\mathrm{rw}}-p_{+}<A$, then the labyrinth $Z$ is $\mathbb{P}$-a.s. non-localised.

(b) Assume that $d \geq 3$. If $1-p_{\mathrm{rw}}-p_{+}<A$, then $Z$ is $\mathbb{P}$-a.s. transient.

As observed after 10.11, we have that $A=A\left(p_{\text {rw }}, d\right) \rightarrow 0$ as $p_{\text {rw }} \downarrow 0$.

Using methods presented in $[115,116,219]$, one may obtain an invariance principle for a random walk in a random labyrinth, under the condition that $1-p_{\mathrm{rw}}-p$ is sufficiently small. Such a principle is valid for a walk which starts in the (a.s) unique infinite equivalence class of $Z$. The details will appear in [74].

The following proof of Theorem 10.17 differs from that presented in $[166]^{8}$. It is slightly more complicated, but gives possibly a better numerical value for the constant $A$.

Proof. The idea is to relate the labyrinth to a certain percolation process, as follows. We begin with the usual lattice $\mathbb{L}^{d}=\left(\mathbb{Z}^{d}, \mathbb{E}^{d}\right)$, and from this we construct the 'line lattice' (or 'covering lattice') $\mathcal{L}$ as follows. The vertex set of $\mathcal{L}$ is the edge-set $\mathbb{E}^{d}$ of $\mathbb{L}^{d}$, and two distinct vertices $e_{1}, e_{2}\left(\in \mathbb{E}^{d}\right)$ of $\mathcal{L}$ are called adjacent in $\mathcal{L}$ if and only if they have a common vertex of $\mathbb{L}^{d}$. If this holds, we write $\left\langle e_{1}, e_{2}\right\rangle$ for the corresponding edge of $\mathcal{L}$, and denote by $\mathbb{F}$ the set of all such edges. We shall work with the graph $\mathcal{L}=\left(\mathbb{E}^{d}, \mathbb{F}\right)$, and shall construct a bond percolation process on $\mathcal{L}$ We may identify $\mathbb{E}^{d}$ with the set of midpoints of members of $\mathbb{E}$; this embedding is We may identify $\mathbb{E}^{d}$ with
useful in visualising $\mathcal{L}$.

Let $\left\langle e_{1}, e_{2}\right\rangle \in \mathbb{E}^{d}$. If the edges $e_{1}$ and $e_{2}$ of $\mathbb{L}^{d}$ are perpendicular, we colour $\left\langle e_{1}, e_{2}\right\rangle$ amber, and if they are parallel blue. Let $0 \leq \alpha, \beta \leq 1$. We declare an edge $\left\langle e_{1}, e_{2}\right\rangle$ of $\mathbb{F}$ to be open with probability $\alpha$ (if amber) or $\beta$ (if blue). This we do for each $\langle e, f\rangle \in \mathbb{F}$ independently of all other members of $\mathbb{F}$. Write $P_{\alpha, \beta}$ for the corresponding probability measure, and let $\theta(\alpha, \beta)$ be the probability that a given vertex $e\left(\in \mathbb{E}^{d}\right)$ of $\mathcal{L}$ is in an infinite open cluster of the ensuing percolation process on $\mathcal{L}$. It is easily seen that $\theta(\alpha, \beta)$ is independent of the choice of $e$.

Lemma 10.18. Let $d \geq 2$ and $0<\alpha \leq 1$. then

$$
\beta_{\mathrm{c}}(\alpha, d)=\sup \{\beta: \theta(\alpha, \beta)=0\}
$$

satisfies $\beta_{\mathrm{c}}(\alpha, d)<1$

Note that, when $d=2$ and $\beta=0$, the process is isomorphic to bond percolation on $\mathbb{L}^{2}$ with edge-parameter $\alpha$. Therefore $\theta(\alpha, 0)>0$ and $\beta_{\mathrm{c}}(\alpha, 2)=0$ when $\alpha>\frac{1}{2}$.

Proof. Since $\beta_{\mathrm{c}}(\alpha, d)$ is non-increasing in $d$, it suffices to prove the conclusion when $d=2$. Henceforth assume that $d=2$.

Here is a sketch proof. Let $L \geq 1$ and let $A_{L}$ be the event that every vertex of $\mathcal{L}$ lying within the box $B\left(L+\frac{1}{2}\right)=\left[-L-\frac{1}{2}, L+\frac{1}{2}\right]^{2}\left(\right.$ of $\left.\mathbb{R}^{2}\right)$ is joined to every other vertex lying within $B\left(L+\frac{1}{2}\right)$ by open paths of $\mathcal{L}$ lying inside $B\left(L+\frac{1}{2}\right)$ which do not use boundary edges. For a given $\alpha$ satisfying $0<\alpha<1$, there exist $L$ and $\beta^{\prime}$ such that

$$
P_{\alpha, \beta^{\prime}}\left(A_{L}\right) \geq p_{\mathrm{c}}(\text { site }),
$$

where $p_{\mathrm{c}}($ site $)$ is the critical probability of site percolation on $\mathbb{L}^{2}$. Now tile $\mathbb{Z}^{2}$ with copies of $B\left(L+\frac{1}{2}\right)$, overlapping at the sides. It follows from the obvious relationship with site percolation that, with positive probability, the origin lies in an infinite cluster.

We now construct a labyrinth on $\mathbb{Z}^{d}$ from each realisation $\omega \in\{0,1\}^{\mathbb{F}}$ of the percolation process (where we write $\omega(f)=1$ if and only if the edge $f$ is open). That is, with each point $x \in \mathbb{Z}^{d}$ we shall associate a member $\rho_{x}$ of $\mathcal{R} \cup\{\varnothing\}$, in such a way that $\rho_{x}$ depends only on the edges $\left\langle e_{1}, e_{2}\right\rangle$ of $\mathbb{F}$ for which $e_{1}$ and $e_{2}$ are distinct edges of $\mathbb{L}^{d}$ having common vertex $x$. It will follow that the collection $\left\{\rho_{x}: x \in \mathbb{Z}^{d}\right\}$ is a family of independent and identically distributed objects.

Since we shall define the $\rho_{x}$ according to a translation-invariant rule, it will suffice to present only the definition of the reflector $\rho_{0}$ at the origin. Let $\mathbb{E}_{0}$ be the set of edges of $\mathbb{L}^{d}$ which are incident to the origin. There is a natural one-one correspondence between $\mathbb{E}_{0}$ and $I^{ \pm}$, namely, the edge $\langle 0, u\rangle$ corresponds to the unit vector $u \in I^{ \pm}$. Let $\rho \in \mathcal{R}$. Using the above correspondence, we may associate with 
$\rho$ a set of configurations in $\Omega=\{0,1\}^{\mathbb{F}}$, as follows. Let $\Omega(\rho, 0)$ be the subset of $\Omega$ containing all configurations $\omega$ satisfying

$$
\omega\left(\left\langle 0, u_{1}\right\rangle,\left\langle 0, u_{2}\right\rangle\right)=1 \quad \text { if and only if } \rho\left(-u_{1}\right)=u_{2}
$$

for all distinct pairs $u_{1}, u_{2} \in I^{ \pm}$

It is not difficult to see that

$$
\Omega\left(\rho_{1}, 0\right) \cap \Omega\left(\rho_{2}, 0\right)=\varnothing \quad \text { if } \quad \rho_{1}, \rho_{2} \in \mathcal{R}, \rho_{1} \neq \rho_{2} .
$$

Let $\omega \in \Omega$ be a percolation configuration on $\mathbb{F}$. We define the reflector $\rho_{0}=\rho_{0}(\omega)$ at the origin by

$$
\rho_{0}= \begin{cases}\rho & \text { if } \omega \in \Omega(\rho, 0) \\ \varnothing & \text { if } \omega \notin \bigcup_{\rho \in \mathcal{R}} \Omega(\rho, 0) .\end{cases}
$$

If $\rho_{0}=\rho \in \mathcal{R}$, then the behaviour of a light beam striking the origin behave as in the corresponding percolation picture, in the following sense. Suppose light is incident in the direction $u_{1}$. There exists at most one direction $u_{2}\left(\neq u_{1}\right)$ such that $\omega\left(\left\langle 0,-u_{1}\right\rangle,\left\langle 0, u_{2}\right\rangle\right)=1$. If such a $u_{2}$ exists, then the light is reflected in this direction. If no such $u_{2}$ exists, then it is reflected back on itself, i.e., in the direction $-u_{1}$.

For $\omega \in\{0,1\}^{\mathbb{F}}$, the above construction results in a random labyrinth $L(\omega)$. If the percolation process contains an infinite open cluster, then the corresponding labyrinth contains (a.s.) an infinite equivalence class.

Turning to probabilities, it is easy to see that, for $\rho \in \mathcal{R}$,

$$
\pi(\rho ; \alpha, \beta)=P_{\alpha, \beta}\left(\rho_{0}=\rho\right)
$$

satisfies $\pi(\rho ; \alpha, \beta)>0$ if $0<\alpha, \beta<1$, and furthermore

$$
\pi(+; \alpha, \beta)=\beta^{d}(1-\alpha)^{\left(\begin{array}{c}
2 d \\
2
\end{array}\right)-d} .
$$

Also,

$$
\pi(\varnothing ; \alpha, \beta)=P_{\alpha, \beta}\left(\rho_{0}=\varnothing\right)=1-\sum_{\rho \in \mathcal{R}} \pi(\rho ; \alpha, \beta) .
$$

Let $p_{\mathrm{rw}}, p_{+}$satisfy $p_{\mathrm{rw}}, p_{+}>0, p_{\mathrm{rw}}+p_{+} \leq 1$. We pick $\alpha, \beta$ such that $0<\alpha, \beta<1$ $\beta>\beta_{\mathrm{c}}(\alpha, 2)$ and

$$
\pi(+; \alpha, \beta) \geq 1-p_{\mathrm{rw}}\left(\geq p_{+}\right) .
$$

(That this may be done is a consequence of the fact that $\beta_{\mathrm{c}}(\alpha, 2)<1$ for all $\alpha>0$; f. Lemma 10.18.)

With this choice of $\alpha, \beta$, let

$$
A=\min \left\{\frac{\pi(\rho ; \alpha, \beta)}{\pi(\rho)}: \rho \neq+, \rho \in \mathcal{R}\right\}
$$

with the convention that $1 / 0=\infty$. (Thus defined, $A$ depends on $\pi$ as well as on $p_{\text {rw }}$. If we set $A=\min \{\pi(\rho ; \alpha, \beta): \rho \neq+, \rho \in \mathcal{R}\}$, we obtain a (smaller) constant which is independent of $\pi$, and we may work with this definition instead.) Then

$$
\pi(\rho ; \alpha, \beta) \geq A \pi(\rho) \text { for all } \rho \neq+,
$$

and in particular

$$
\pi(\rho ; \alpha, \beta) \geq\left(1-p_{\mathrm{rw}}-p_{+}\right) \pi(\rho) \quad \text { if } \quad \rho \neq+
$$

so long as $p_{+}$satisfies $1-p_{\mathrm{rw}}-p_{+}<A$. Note that $A=A(\alpha, \beta)>0$.

We have from the fact that $\beta>\beta_{\mathrm{c}}(\alpha, 2)$ that the percolation process $\omega$ a.s. contains an infinite open cluster. It follows that there exists a.s. a rw point in $L(\omega)$ which is $L(\omega)$-non-localised. The labyrinth $Z$ of the theorem may be obtained (in distribution) from $L$ as follows. Having sampled $L(\omega)$, we replace any crossing (resp. reflector $\rho(\neq+)$ ) by a rw point with probability $\pi(+; \alpha, \beta)-p_{+}$(resp. $\left.\pi(\rho ; \alpha, \beta)-\left(1-p_{\mathrm{rw}}-p_{+}\right) \pi(\rho)\right) ;$ cf. $(10.20)$ and (10.21). The ensuing labyrinth $L^{\prime}(\omega)$ has the same probability distribution as $Z$. Furthermore, if $L(\omega)$ is non-localised, then so is $L^{\prime}(\omega)$.

The first part of Theorem 10.17 has therefore been proved. Assume henceforth that $d \geq 3$, and consider part (b).

Now consider a labyrinth defined by $p_{\mathrm{rw}}, p_{+}, \pi(\cdot)$. Let $e$ be an edge of $\mathbb{Z}^{d}$. Either $e$ lies in a unique path joining two rw points (but no other rw point) of some length $l(e)$, or it does not (in which case we set $l(e)=0)$. Now, the random walk in thi abyrinth induces an embedded Markov chain on the set of rw points. This chai corresponds to an electrical network obtained by placing an electrical resistor at each edge $e$ having resistance $l(e)^{-1}$. We now make two comparisons, the effect of each of which is to increase all effective resistances of the network. At the first stage, we replace all finite edge-resistances $l(e)^{-1}$ by unit resistances. This cannot decrease any effective resistance. At the next stage we replace each $\mathrm{rw}$ point by

$+\quad$ with probability $\pi(+; \alpha, \beta)-p_{+}$

$$
\rho(\neq+) \quad \text { with probability } \pi(\rho ; \alpha, \beta)-\left(1-p_{\mathrm{rw}}-p_{+}\right) \pi(\rho),
$$

in accordance with (10.20) and (10.21) (and where $\alpha, \beta$ are chosen so that (10.20), (10.21) hold, and furthermore $\left.\beta_{\mathrm{c}}(\alpha, 2)<\beta<1\right)$. Such replacements can only increase effective resistance.

In this way we obtain a comparison between the resistance of the network arising from the above labyrinth and that of the labyrinth $L(\omega)$ defined around (10.19). Indeed, it suffices to prove that the effective resistance between 0 and $\infty$ (in the infinite equivalence class) of the labyrinth $L(\omega)$ is a.s. finite. By examining the geometry, we claim that this resistance is no greater (up to a multiplicative constant) than the resistance between the origin and infinity of the corresponding infinite open percolation cluster of $\omega$. By the next lemma, the last resistance is a.s. finite, whence the original walk is a.s. transient (when confined to the almost surely unique infinite equivalence class). 
Lemma 10.22. Let $d \geq 3,0<\alpha<1$, and $\beta_{\mathrm{c}}(\alpha, 2)<\beta<1$. Let $R$ be the effective resistance between the origin and the points at infinity, in the above bond percolation process $\omega$ on $\mathcal{L}$. Then

$$
P_{\alpha, \beta}(R<\infty \mid 0 \text { belongs to the infinite open cluster })=1 .
$$

Presumably the same conclusion is valid under the weaker hypothesis that $\beta>$ $\beta_{\mathrm{c}}(\alpha, d)$.

Sketch Proof. Rather than present all the details, here are some notes. The main techniques used in [164] arise from [165], and principally one uses the exponential decay noted in Lemma 10.4. That such decay is valid whenever $\beta>\beta_{\mathrm{c}}(\alpha, d)$ uses the machinery of [165]. This machinery may be developed in the present settin (in [165] it is developed only for the hypercubic lattice $\mathbb{L}^{d}$ ). Alternatively, 'slab arguments' show (a) and (b) of Lemma 10.4 for sufficiently large $\beta$; certainly the condition $\beta>\beta_{\mathrm{c}}(\alpha, 2)$ suffices for the conclusion.

Comments on the Proof of Lemma 10.16. This resembles closely the proof of the uniqueness of the infinite percolation cluster (Theorem 7.1). We do not give the details. The notion of 'trifurcation' is replaced by that of an 'encounter zone'. Let $R \geq 1$ and $B=B(R)$. A translate $x+B$ is called an encounter zone if

(a) all points in $x+B$ are rw points, and

(b) in the labyrinth $\mathbb{Z}^{d} \backslash\{x+B\}$, there are three or more infinite equivalence classes which are part of the same equivalence class of $\mathbb{L}^{d}$.

Note that different encounter zones may overlap. See [74] for more details.

\section{FRACTAL PERCOLATION}

11.1. RANDOM FRACTALS

Many so called 'fractals' are generated by iterative schemes, of which the classical middle-third Cantor construction is a canonical example. When the scheme incoporates a randomised step, then the ensuing set may be termed a 'random fractal'. Such sets may be studied in some generality (see [131, 153, 183, 313]), and propertie of fractal dimension may be established. The following simple example is directed at a 'percolative' property, namely the possible existence in the random fractal of long paths.

We begin with the unit square $C_{0}=[0,1]^{2}$. At the first stage, we divide $C_{0}$ into nine (topologically closed) subsquares of side-length $\frac{1}{3}$ (in the natural way), and we declare each of the subsquares to be open with probability $p$ (independently of any ther subsquare). Write $C_{1}$ for the union of the open subsquares thus obtained. We now iterate this construction on each subsquare in $C_{1}$, obtaining a collection of open (sub)subsquares of side-length $\frac{1}{9}$. After $k$ steps we have obtained a union $C_{k}$ of open squares of side-length $\left(\frac{1}{3}\right)^{k}$. The limit set

$$
C=\lim _{k \rightarrow \infty} C_{k}=\bigcap_{k \geq 1} C_{k}
$$

is a random set whose metrical properties we wish to study. See Figure 11.1.

Constructions of the above type were introduced by Mandelbrot [254] and initially studied by Chayes, Chayes, and Durrett [92]. Recent papers include [114, 134, 302]. Many generalisations of the above present themselves.

a) Instead of working to base 3 , we may work to base $M$ where $M \geq 2$

(b) Replace two dimensions by $d$ dimensions where $d \geq 2$.

c) Generalise the use of a square.

In what follows, (a) and (b) are generally feasible, while (c) poses a different circle of problems.

It is easily seen that the number $X_{k}$ of squares present in $C_{k}$ is a branching process with family-size generating function $G(x)=(1-p+p x)^{9}$. Its extinction probability $\eta$ is a root of the equation $\eta=G(\eta)$, and is such that

$$
P_{p}(\text { extinction }) \begin{cases}=1 & \text { if } p \leq \frac{1}{9} \\ <1 & \text { if } p>\frac{1}{9}\end{cases}
$$

Therefore

$$
P_{p}(C=\varnothing)=1 \quad \text { if and only if } \quad p \leq \frac{1}{9} .
$$

When $p>\frac{1}{9}$, then $C$ (when non-extinct) is large but ramified. 

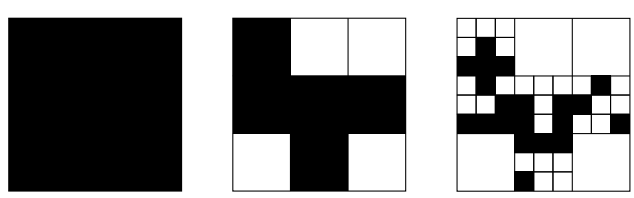

Fig. 11.1. Three stages in the construction of the 'random Cantor set' $C$. At each stage, a square is replaced by a $3 \times 3$ grid of smaller squares, each of which is retained with probability $p$.

Theorem 11.3. Let $p>\frac{1}{9}$. The Hausdorff dimension of $C$, conditioned on the event $\{C \neq \varnothing\}$, equals a.s. $\log (9 p) / \log 3$.

Rather than prove this in detail, we motivate the answer. The set $C$ is covered by $X_{k}$ squares of side-length $\left(\frac{1}{3}\right)^{k}$. Therefore the $\delta$-dimensional box measure $H_{\delta}(C)$ satisfies

$$
H_{\delta}(C) \leq X_{k} 3^{-k \delta} \text {. }
$$

Conditional on $\{C \neq \varnothing\}$, the random variables $X_{k}$ satisfy

$$
\frac{\log X_{k}}{k} \rightarrow \log \mu \quad \text { as } \quad k \rightarrow \infty, \quad \text { a.s. }
$$

where $\mu=9 p$ is the mean family-size of the branching process. Therefore

$$
H_{\delta}(C) \leq(9 p)^{k(1+\mathrm{o}(1))} 3^{-k \delta} \quad \text { a.s. }
$$

which tends to 0 as $k \rightarrow \infty$ if

$$
\delta>\frac{\log (9 p)}{\log 3} .
$$

It follows that the box dimension of $C$ is (a.s.) no larger than $\log (9 p) / \log 3$. Experts may easily show that this bound for the dimension of $C$ is (a.s.) exact on the event that $C \neq \varnothing($ see $[131,183,313])$.

Indeed the exact Hausdorff measure function of $C$ may be ascertained (see [153]), and is found to be $h(t)=t^{d}(\log |\log t|)^{1-\frac{1}{2} d}$ where $d$ is the Hausdorff dimension of

\subsection{Percolation}

Can $C$ contain long paths? More concretely, can $C$ contain a crossing from left to right of the original unit square $C_{0}$ (which is to say that $C$ contains a connected subset which has non-trivial intersection with the left and right sides of the unit square)? Let LR denote the event that such a crossing exists in $C$, and define the percolation probability

(11.4)

$$
\theta(p)=P_{p}(\mathrm{LR})
$$

In [92], it was proved that there is a non-trivial critical probability

$$
p_{\mathrm{c}}=\sup \{p: \theta(p)=0\} .
$$

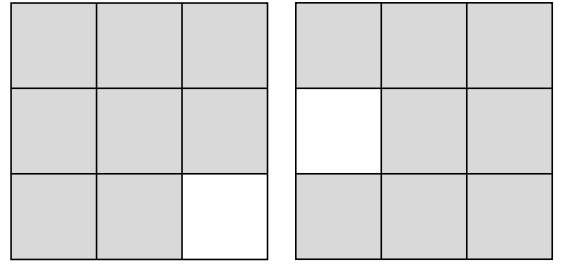

Fig. 11.2. The key fact of the construction is the following. Whenever two large squares abut, and each has the property that at least 8 of its subsquares are retained, then their union contains a crossing from the left side to the right side.

Theorem 11.5. We have that $0<p_{\mathrm{c}}<1$, and furthermore $\theta\left(p_{\mathrm{c}}\right)>0$.

Partial Proof. This proof is taken from [92] with help from [114]. Clearly $p_{\mathrm{c}} \geq \frac{1}{9}$, and we shall prove next that

$$
p_{\mathrm{c}} \leq \frac{8}{9}\left(\frac{64}{63}\right)^{7} \simeq 0.99248 \ldots
$$

Write $\mathcal{C}=\left(C_{0}, C_{1}, \ldots\right)$. We call $\mathcal{C} 1$-good if $\left|C_{1}\right| \geq 8$. More generally, we call $\mathcal{C}$ $(k+1)$-good if at least 8 of the squares in $C_{1}$ are $k$-good. The following fact is crucial for the argument: if $\mathcal{C}$ is $k$-good then $C_{k}$ contains a left-right crossing of the unit square (see Figure 11.2). Therefore (using the fact that the limit of a decreasing sequence of compact connected sets is connected, and a bit more ${ }^{9}$ )

$(11.6$

$$
P_{p}(\mathcal{C} \text { is } k \text {-good }) \leq P_{p}\left(C_{k} \text { crosses } C_{0}\right) \downarrow \theta(p) \quad \text { as } k \rightarrow \infty,
$$

whence it suffices to find a value of $p$ for which $\pi_{k}=\pi_{k}(p)=P_{p}(\mathcal{C}$ is $k$-good) satisfies

$$
\pi_{k}(p) \rightarrow \pi(p)>0 \quad \text { as } \quad k \rightarrow \infty .
$$

We define $\pi_{0}=1$. By an easy calculation,

$$
\pi_{1}=9 p^{8}(1-p)+p^{9}=F_{p}\left(\pi_{0}\right)
$$

where

$$
F_{p}(x)=p^{8} x^{8}(9-8 p x)
$$

More generally

$$
\pi_{k+1}=F_{p}\left(\pi_{k}\right)
$$

${ }^{9}$ There are some topological details which are necessary for the limit in (11.6). Look at the set $\mathcal{S}_{k}$ of maximal connected components of $C_{k}$ which intersect the left and right sides of $C_{0}$. Thes that member. On the event $\left\{C\right.$ crosses $\left.C_{0}\right\}$, the ensuing family tree has finite vertex degrees and contains an infinite path $S_{1}, S_{2} \ldots$ of compact connected sets. The intersection $S_{\infty}=\lim _{k \rightarrow \infty} S_{k}$ is non-empty and connected. By a similar argument, $S_{\infty}$ has non-trivial intersections with the left and right sides of $C_{0}$. It follows that $\left\{C_{k}\right.$ crosses $\left.C_{0}\right\} \downarrow\left\{C\right.$ crosses $\left.C_{0}\right\}$, as required in (11.6). Part of this argument was suggested by Alan Stacey. 


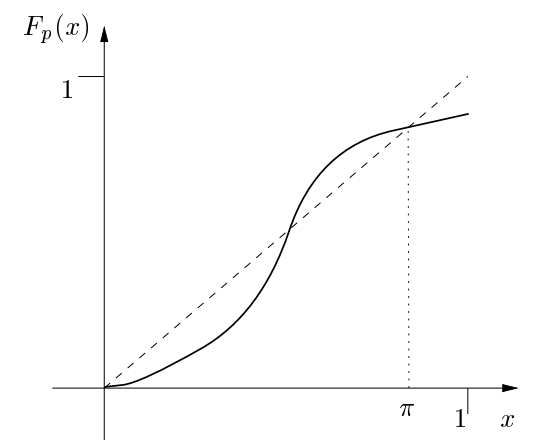

Fig. 11.3. A sketch of the function $F_{p}$ for $p$ close to 1, with the largest fixed point $\pi$ marked.

About the function $F_{p}$ we note that $F_{p}(0)=0, F_{p}(1)<1$, and

$$
F_{p}^{\prime}(x)=72 p^{8} x^{7}(1-p x) \geq 0 \quad \text { on } \quad[0,1] .
$$

See Figure 11.3 for a sketch of $F_{p}$.

It follows that $\pi_{k} \downarrow \pi$ as $k \rightarrow \infty$ where $\pi$ is the largest fixed point of $F_{p}$ in the interval $[0,1]$.

It is elementary that $F_{p_{0}}\left(x_{0}\right)=x_{0}$, where

$$
x_{0}=\frac{9}{8}\left(\frac{63}{64}\right)^{8}, \quad p_{0}=\frac{8}{9}\left(\frac{64}{63}\right)^{7} .
$$

It follows that $\pi\left(p_{0}\right) \geq x_{0}$, yielding $\theta\left(p_{0}\right)>0$. Therefore $p_{\mathrm{c}} \leq p_{0}$, as required in $(11.5)$

The proof that $\theta\left(p_{\mathrm{c}}\right)>0$ is more delicate; see [114]

Consider now the more general setting in which the random step involves replacing a typical square of side-length $M^{-k}$ by a $M \times M$ grid of subsquares of common side-length $M^{-(k+1)}$ (the above concerns the case $M=3$ ). For general $M$, a version of the above argument yields that the corresponding critical probability $p_{\mathrm{c}}(M)$ satisfies $p_{\mathrm{c}}(M) \geq M^{-2}$ and also

$$
p_{\mathrm{c}}(M)<1 \quad \text { if } M \geq 3 \text {. }
$$

When $M=2$, we need a special argument in order to obtain that $p_{\mathrm{c}}(2)<1$, and this may be achieved by using the following coupling of the cases $M=2$ and $M=4$ (see $[92,114]$ ). Divide $C_{0}$ into a $4 \times 4$ grid and do as follows. At the first stage, with probability $p$ we retain all four squares in the top left corner; we do similarly for the three batches of four squares in each of the other three corner of $C_{0}$. Now for the second stage: examine each subsquare of side-length $\frac{1}{4}$ so far retained, and delete such a subsquare with probability $p$ (different subsquares being treated independently). Note that the probability measure at the first stage dominates (stochastically) product measure with intensity $\pi$ so long as $(1-\pi)^{4} \geq$ $1-p$. Choose $\pi$ to satisfy equality here. The composite construction outlined above dominates (stochastically) a single step of a $4 \times 4$ random fractal with parameter $p \pi=p\left(1-(1-p)^{\frac{1}{4}}\right)$, which implies that

$$
p_{\mathrm{c}}(2)\left(1-\left(1-p_{\mathrm{c}}(2)\right)^{\frac{1}{4}}\right) \leq p_{\mathrm{c}}(4)
$$

and therefore $p_{\mathrm{c}}(2)<1$ by $(11.10)$.

\subsection{A Morphology}

Random fractals have many phases, of which the existence of left-right crossings characterises only one. A weaker property than the existence of crossings is that the projection of $C$ onto the $x$-axis is the whole interval $[0,1]$. Projections of random fractals are of independent interest (see, for example, the 'digital sundial' theorem of [132]). Dekking and Meester [114] have cast such properties within a more general morphology.

We write $C$ for a random fractal in $[0,1]^{2}$ (such as that presented in Section 11.1). The projection of $C$ is denoted as

$$
\pi C=\{x \in \mathbb{R}:(x, y) \in C \text { for some } y\},
$$

and $\lambda$ denotes Lebesgue measure. We say that $C$ lies in one of the following phase if it has the stated property. A set is said to percolate if it contains a left-right crossing of $[0,1]^{2}$; dimension is denoted by 'dim'.

I. $C=\varnothing$ a.s.

II. $P(C \neq \varnothing)>0, \operatorname{dim}(\pi C)=\operatorname{dim} C$ a.s.

III. $\operatorname{dim}(\pi C)<\operatorname{dim} C$ a.s. on $\{C \neq \varnothing\}$, but $\lambda(\pi C)=0$ a.s.

IV. $0<\lambda(\pi C)<1$ a.s. on $\{C \neq \varnothing\}$.

V. $P(\lambda(\pi C)=1)>0$ but $C$ does not percolate a.s.

I. $P(C$ percolates $)>0$

In many cases of interest, there is a parameter $p$, and the ensuing fractal moves through the phases, from I to VI, as $p$ increases from 0 to 1 . There may be critical values $p_{M, N}$ at which the model moves from Phase $M$ to Phase $N$. In a variety of cases, the critical values $p_{\mathrm{I}, \mathrm{II}}, p_{\mathrm{II}, \mathrm{III}}, p_{\mathrm{III}, \mathrm{IV}}$ can be determined exactly, whereas $p_{\mathrm{IV}, \mathrm{V}}$ and $p_{\mathrm{V}, \mathrm{VI}}$ can be much harder to find.

Here is a reasonably large family of random fractals. As before, they are constructed by dividing a square into 9 equal subsquares. In this more general system, we are provided with a probability measure $\mu$, and we replace a square by the union of a random collection of subsquares sampled according to $\mu$. This process is iterated on all relevant scales. Certain parameters are especially relevant. Let $\sigma_{l}$ be the number of subsquares retained from the $l$ th column, and let $m_{l}=E\left(\sigma_{l}\right)$ be it mean 

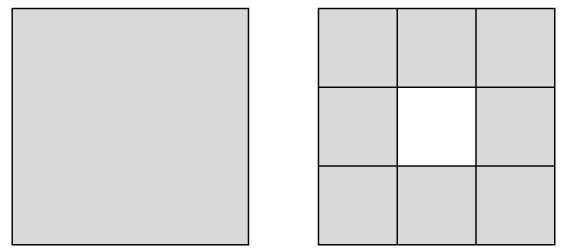

11.4. In the construction of the Sier pinski carpet, the middle square is always deleted.

Theorem 11.11. We have that

(a) $C=\varnothing$ if and only if $\sum_{l=1}^{3} m_{l} \leq 1$ (unless some $\sigma_{l}$ is a.s. equal to 1 ),

(b) $\operatorname{dim}(\pi C)=\operatorname{dim}(C)$ a.s. if and only if

$$
\sum_{l=1}^{3} m_{l} \log m_{l} \leq 0
$$

(c) $\lambda(\pi C)=0$ a.s. if and only if

$$
\sum_{l=1}^{3} \log m_{l} \leq 0
$$

For the proofs, see $[113,134]$. Consequently, one may check the Phases I, II, III by a knowledge of the $m_{l}$ only.

Next we apply Theorem 11.11 to the random Cantor set of Section 11.1, to obtain that, for this model, $p_{\mathrm{I}, \mathrm{I}}=\frac{1}{9}$, and we depart Phase II as $p$ increases through the value $\frac{1}{3}$. The system is never in Phase III (by Theorem 11.1(c)) or in Phase IV (by Theorem 1 of [134]). It turns out that $p_{\mathrm{II}, \mathrm{V}}=\frac{1}{3}$ and $\frac{1}{2}<p_{\mathrm{V}, \mathrm{VI}}<1$.

For the 'random Sierpinski carpet' (RSC) the picture is rather different. Thi model is constructed as the above but with one crucial difference: at each iteration, the central square is removed with probability one, and the others with probability $1-p$ (see Figure 11.4). Applying Theorem 11.11 we find that

$$
p_{\mathrm{I}, \mathrm{II}}=\frac{1}{8}, \quad p_{\mathrm{II}, \mathrm{III}}=54^{-\frac{1}{4}}, \quad p_{\mathrm{III}, \mathrm{IV}}=18^{-\frac{1}{3}},
$$

and it happens that

$$
\frac{1}{2}<p_{\mathrm{IV}, \mathrm{V}} \leq 0.8085, \quad 0.812 \leq p_{\mathrm{V}, \mathrm{VI}} \leq 0.991 .
$$

See $[114]$ for more details

We close this section with a conjecture which has received some attention. Writing $p_{\mathrm{c}}$ (resp. $p_{\mathrm{c}}(\mathrm{RSC})$ ) for the critical point of the random fractal of Section 11.1 (resp. the random Sierpinski carpet), it is evident that $p_{\mathrm{c}} \leq p_{\mathrm{c}}$ (RSC). Prove or disprove the strict inequality $p_{\mathrm{c}}<p_{\mathrm{c}}$ (RSC)
11.4 Relationship to Brownian Motion

Peres [312] has discovered a link between fractal percolation and Brownian Motion, via a notion called 'intersection-equivalence'. For a region $U \subseteq \mathbb{R}^{d}$, we call two andom sets $B$ and $C$ intersection-equivalent in $U$ if

$$
P(B \cap \Lambda \neq \varnothing) \asymp P(C \cap \Lambda \neq \varnothing) \text { for all closed } \Lambda \subseteq U
$$

(i.e., there exist positive finite constants $c_{1}, c_{2}$, possibly depending on $U$, such that

$$
c_{1} \leq \frac{P(B \cap \Lambda \neq \varnothing)}{P(C \cap \Lambda \neq \varnothing)} \leq c_{2}
$$

for all closed $\Lambda \subseteq U)$.

We apply this definition for two particular random sets. First, write $B$ for the range of Brownian Motion in $\mathbb{R}^{d}$, starting at a point chosen uniformly at random in the unit cube. Also, for $d \geq 3$, let $C$ be a random Cantor set constructed by binary splitting (rather than the ternary splitting of Section 11.1) and with parameter $p=2^{2-d}$.

Theorem 11.13. Suppose that $d \geq 3$. The random sets $B$ and $C$ are intersectionequivalent.

A similar result is valid when $d=2$, but with a suitable redefinition of the random set $C$. This is achieved by taking different values of $p$ at the different stages of the construction, namely $p=k /(k+1)$ at the $k$ th stage.

This correspondence is not only beautiful and surprising, but also useful. It provides a fairly straightforward route to certain results concerning intersections of random walks and Brownian Motions, for example. Conversely, using the rotationinvariance of Brownian Motion, one may obtain results concerning projections of the random Cantor set in other directions than onto an axis (thereby complementing results of [113], in the case of the special parameter-value given above).

The proof of Theorem 11.13 is analytical, and proceeds by utilising

- classical potential theory for Brownian Motion,

- the relationship between capacity and percolation for trees ([249]), and

- the relationship between capacity on trees and capacity on an associated Euclidean space ([55, 309]).

It is an attractive target to understand Theorem 11.13 via a coupling of the two random sets. 


\section{ISING AND POTTS MODELS}

12.1 Ising Model For Ferromagnets

In a famous experiment, a piece of iron is exposed to a magnetic field. The field increases from zero to a maximum, and then diminishes to zero. If the temperature is sufficiently low, the iron retains some 'residual magnetisation', otherwise it does not. The critical temperature for this phenomenon is often called the Curie point In a famous scientific paper [194], Ising proposed a mathematical model which may be phrased in the following way, using the modern idiom.

Let $\Lambda$ be a box of $\mathbb{Z}^{d}$, say $\Lambda=[-n, n]^{d}$. Each vertex in $\Lambda$ is allocated a random spin according to a Gibbsian probability measure as follows. Since spins come in two basic types, we take as sample space the set $\Sigma_{\Lambda}=\{-1,+1\}^{\Lambda}$, and we consider the probability measure $\pi_{\Lambda}$ which allocates a probability to a spin vector $\sigma \in \Sigma_{\Lambda}$ given by

$$
\pi_{\Lambda}(\sigma)=\frac{1}{Z_{\Lambda}} \exp \left\{-\beta H_{\Lambda}(\sigma)\right\}, \quad \sigma \in \Sigma_{\Lambda},
$$

where $\beta=T^{-1}$ (the reciprocal of temperature, on a certain scale) and the Hamiltonian $H_{\Lambda}: \Sigma_{\Lambda} \rightarrow \mathbb{R}$ is given by

$$
H_{\Lambda}(\sigma)=-\sum_{e=\langle i, j\rangle} J_{e} \sigma_{i} \sigma_{j}-h \sum_{i} \sigma_{i}
$$

for constants $\left(J_{e}\right)$ and $h$ (called the 'external field') which parameterise the process. The sums in (12.2) are over all edges and vertices of $\Lambda$, respectively.

The measure (12.1) is said to arise from 'free boundary conditions', since the boundary spins have no special role. It turns out to be interesting to allow other types of boundary conditions. For any assignment $\gamma: \partial \Lambda \rightarrow\{-1,+1\}$ there is a corresponding probability measure $\pi_{\Lambda}^{\gamma}$ obtained by restricting the vector $\sigma$ to the set of vectors which agree with $\gamma$ on $\partial \Lambda$. In this way we may obtain measures $\pi_{\Lambda}^{+}$ $\pi_{\Lambda}^{-}$, and $\pi_{\Lambda}^{\mathrm{f}}$ (with free boundary conditions) on appropriate subsets of $\Sigma_{\Lambda}$

For simplicity, we assume here that $J_{e}=J>0$ for all edges $e$. In this 'ferromagnetic' case, measures of the form (12.1) prefer to see configurations $\sigma$ in which neighbouring vertices have like spins. The antiferromagnetic case $J<0$ can be somewhat tricky.

Inspecting (12.1)-(12.2) with $J>0$, we see that spins tend to align with the sign of any external field $h$.

The following questions are basic.

(a) What weak limits $\lim _{\Lambda \rightarrow \mathbb{Z} d} \pi_{\Lambda}^{\gamma}$ exist for possible boundary conditions $\gamma$ ? (This requires redefining $\pi_{\Lambda}^{\gamma}$ as a probability measure associated with the sample space $\Sigma=\{-1,+1\}^{\mathbb{Z}^{d}}$.)

(b) Under what conditions on $J, h, d$ is there a unique limit measure?

(c) How may limit measures be characterised?

d) What are their properties; for example, at what rate do their correlations decay over large distances? (e) Is there a phase transition?

It turns out that there is a unique limit if either $d=1$ or $h \neq 0$. There is non-uniqueness when $d \geq 2, h=0$, and $\beta$ is sufficiently large (i.e., $\beta>T_{\mathrm{c}}^{-1}$ where $T_{\mathrm{c}}$ is the Curie point).

A great deal is known about the Ising model; see, for example, $[9,130,135,150$, $236]$ and many other sources. We choose here to follow a random-cluster analysis, the details of which will follow.

The Ising model on $\mathbb{L}^{2}$ permits one of the famous exact calculations of statistical physics, following Onsager [300].

\subsection{Potts Models}

Whereas the Ising model permits two possible spin-values at each vertex, the Potts model permits a general number $q \in\{2,3, \ldots\}$. The model was introduced by Pott [318] following an earlier paper of Ashkin and Teller [46]

Let $q$ be an integer, at least 2 , and take as sample space $\Sigma_{\Lambda}=\{1,2, \ldots, q\}^{\Lambda}$ where $\Lambda$ is given as before. This time we set

$$
\pi_{\Lambda}(\sigma)=\frac{1}{Z_{\Lambda}} \exp \left\{-\beta H_{\Lambda}(\sigma)\right\}, \quad \sigma \in \Sigma_{\Lambda},
$$

where

$$
H_{\Lambda}(\sigma)=-J \sum_{e=\langle i, j\rangle} \delta_{\sigma_{i}, \sigma_{j}}
$$

and $\delta_{u, v}$ is the Kronecker delta

$$
\delta_{u, v}= \begin{cases}1 & \text { if } u=v \\ 0 & \text { otherwise. }\end{cases}
$$

External field is absent from this formulation, but can be introduced if required by the addition to (12.4) of the term $-h \sum_{i} \delta_{\sigma_{i}, 1}$, which favours an arbitrarily chosen spin-value, being here the value 1 .

The labelling $1,2, \ldots, q$ of the spin-values is of course arbitrary. The case $q=2$ is identical to the Ising model (without external field and with an amended value of $J)$, since

$$
\sigma_{i} \sigma_{j}=2 \delta_{\sigma_{i}, \sigma_{j}}-1 \quad \text { for } \sigma_{i}, \sigma_{j} \in\{-1,+1\} .
$$




\subsection{RANDOM-Cluster MODELS}

It was Fortuin and Kasteleyn who discovered that Potts models may be recast as 'random-cluster models'. In doing so, they described a class of models, including percolation, which merits attention in their own right, and through whose analysis we discover fundamental facts concerning Ising and Potts models. See [159] for a recent account of the relevant history and bibliography.

The neatest construction of random-cluster models from Potts models is that reported in [128]. Let $G=(V, E)$ be a finite graph, and define the sample spaces

$$
\Sigma=\{1,2, \ldots, q\}^{V}, \quad \Omega=\{0,1\}^{E},
$$

where $q$ is a positive integer. We now define a probability mass function $\mu$ on $\Sigma \times \Omega$

$$
\mu(\sigma, \omega) \propto \prod_{e \in E}\left\{(1-p) \delta_{\omega(e), 0}+p \delta_{\omega(e), 1} \delta_{e}(\sigma)\right\}
$$

where $0 \leq p \leq 1$, and

$$
\delta_{e}(\sigma)=\delta_{\sigma_{i}, \sigma_{j}} \quad \text { if } e=\langle i, j\rangle \in E .
$$

Elementary calculations reveal the following facts.

(a) Marginal on $\Sigma$. The marginal measure

$$
\mu(\sigma, \cdot)=\sum_{\omega \in \Omega} \mu(\sigma, \omega)
$$

is given by

$$
\mu(\sigma, \cdot) \propto \exp \left\{\beta J \sum_{e} \delta_{e}(\sigma)\right\}
$$

where $p=1-e^{-\beta J}$. This is the Potts measure (12.3). Note that $\beta J \geq 0$.

(b) Marginal on $\Omega$. Similarly

$$
\mu(\cdot, \omega)=\sum_{\sigma \in \Sigma} \mu(\sigma, \omega) \propto\left\{\prod_{e} p^{\omega(e)}(1-p)^{1-\omega(e)}\right\} q^{k(\omega)}
$$

where $k(\omega)$ is the number of connected components (or 'clusters') of the graph with vertex set $V$ and edge set $\eta(\omega)=\{e \in E: \omega(e)=1\}$.

(c) The conditional measures. Given $\omega$, the conditional measure on $\Sigma$ is obtained by putting (uniformly) random spins on entire clusters of $\omega$ (of which there are $k(\omega)$ ), which are constant on given clusters, and independent between clusters. Given $\sigma$, the conditional measure on $\Omega$ is obtained by setting $\omega(e)=0$ if $\delta_{e}(\sigma)=0$, and otherwise $\omega(e)=1$ with probability $p$ (independently of other edges).
In conclusion, the measure $\mu$ is a coupling of a Potts measure $\pi_{\beta, J}$ on $V$, together with a 'random-cluster measure'

$$
\phi_{p, q}(\omega) \propto\left\{\prod_{e \in E} p^{\omega(e)}(1-p)^{1-\omega(e)}\right\} q^{k(\omega)}, \quad \omega \in \Omega .
$$

The parameters of these measures correspond to one another by the relation $p=$ $1-e^{-\beta J}$. Since $0 \leq p \leq 1$, this is only possible if $\beta J \geq 0$.

Why is this interesting? The 'two-point correlation function' of the Potts measure $\pi_{\beta, J}$ on $G=(V, E)$ is defined to be the function $\tau_{\beta, J}$ given by

$$
\tau_{\beta, J}(i, j)=\pi_{\beta, J}\left(\sigma_{i}=\sigma_{j}\right)-\frac{1}{q}, \quad i, j \in V .
$$

The 'two-point connectivity function' of the random-cluster measure $\phi$ is $\phi_{p, q}(i \leftrightarrow j)$ i.e., the probability that $i$ and $j$ are in the same cluster of a configuration sampled according to $\phi$. It turns out that these 'two-point functions' are (except for a constant factor) the same.

Theorem 12.8. If $q \in\{2,3, \ldots\}$ and $p=1-e^{-\beta J}$ satisfies $0 \leq p \leq 1$, then

$$
\tau_{\beta, J}(i, j)=\left(1-q^{-1}\right) \phi_{p, q}(i \leftrightarrow j)
$$

Proof. We have that

$$
\begin{aligned}
\tau_{\beta, J}(i, j) & =\sum_{\sigma, \omega}\left\{1_{\left\{\sigma_{i}=\sigma_{j}\right\}}(\sigma)-q^{-1}\right\} \mu(\sigma, \omega) \\
& =\sum_{\omega} \phi_{p, q}(\omega) \sum_{\sigma} \mu(\sigma \mid \omega)\left\{1_{\left\{\sigma_{i}=\sigma_{j}\right\}}(\sigma)-q^{-1}\right\} \\
& =\sum_{\omega} \phi_{p, q}(\omega)\left\{\left(1-q^{-1}\right) 1_{\{i \leftrightarrow j\}}(\omega)+0 \cdot 1_{\{i \leftrightarrow j\}}(\omega)\right\} \\
& =\left(1-q^{-1}\right) \phi_{p, q}(i \leftrightarrow j) .
\end{aligned}
$$

This fundamental correspondence implies that properties of Potts correlation can be mapped to properties of random-cluster connection. Since Pottsian phase transition can be formulated in terms of correlation functions, this implies that information about percolative phase transition for random-cluster models is useful for studying Pottsian transitions. In doing so, we study the 'stochastic geometry' of random-cluster models.

The random-cluster measure (12.7) was constructed under the assumption that $q \in\{2,3, \ldots\}$, but $(12.7)$ makes sense for any positive real $q$. We have therefore obtained a rich family of measures which includes percolation $(q=1)$ as well as the Ising $(q=2)$ and Potts measures. 


\section{RANDOM-CLUSTER MODELS}

13.1 Basic Properties

First we summarise some useful properties of random-cluster measures. Let $G=$ $(V, E)$ be a finite graph, and write $\Omega_{E}=\{0,1\}^{E}$. The random-cluster measure on $\Omega_{E}$, with parameters $p, q$ satisfying $0 \leq p \leq 1$ and $q>0$, is given by

$$
\phi_{p, q}(\omega)=\frac{1}{Z}\left\{\prod_{e \in E} p^{\omega(e)}(1-p)^{1-\omega(e)}\right\} q^{k(\omega)}, \quad \omega \in \Omega_{E}
$$

where $Z=Z_{G, p, q}$ is a normalising constant, and $k(\omega)$ is the number of connected components of the graph $(V, \eta(\omega))$, where $\eta(\omega)=\{e: \omega(e)=1\}$ is the set of 'open' edges.

Theorem 13.1. The measure $\phi_{p, q}$ satisfies the FKG inequality if $q \geq 1$.

Proof. If $p=0,1$, the conclusion is obvious. Assume $0<p<1$, and check the condition (5.2), which amounts to the assertion that

$$
k\left(\omega \vee \omega^{\prime}\right)+k\left(\omega \wedge \omega^{\prime}\right) \geq k(\omega)+k\left(\omega^{\prime}\right) \quad \text { for } \omega, \omega^{\prime} \in \Omega_{E} .
$$

This we leave as a graph-theoretic exercise.

Theorem 13.2 (Comparison Inequalities). We have that

$$
\begin{aligned}
& \phi_{p^{\prime}, q^{\prime}} \leq \phi_{p, q} \quad \text { if } \quad p^{\prime} \leq p, q^{\prime} \geq q, q^{\prime} \geq 1 \\
& \phi_{p^{\prime}, q^{\prime}} \geq \phi_{p, q} \quad \text { if } \quad \frac{p^{\prime}}{q^{\prime}\left(1-p^{\prime}\right)} \geq \frac{p}{q(1-p)}, q^{\prime} \geq q, q^{\prime} \geq 1 .
\end{aligned}
$$

Proof. Use Holley's Inequality (Theorem 5.5) after checking condition (5.6).

In the next theorem, the role of the graph $G$ is emphasised in the use of the notation $\phi_{G, p, q}$. The graph $G \backslash e$ (resp. G.e) is obtained from $G$ by deleting (resp. contracting) the edge $e$

Theorem 13.5 (Tower Property). Let $e \in E$.

(a) Given $\omega(e)=0$, the conditional measure obtained from $\phi_{G, p, q}$ is $\phi_{G \backslash e, p, q}$.

(b) Given $\omega(e)=1$, the conditional measure obtained from $\phi_{G, p, q}$ is $\phi_{G . e, p, q}$.

Proof. This is an elementary calculation of conditional probabilities.

More details of these facts may be found in $[27,160,163]$. Another comparison inequality may be found in [162].
13.2 Weak Limits and Phase Transitions

Let $d \geq 2$, and $\Omega=\{0,1\}^{\mathbb{E}^{d}}$. The appropriate $\sigma$-field of $\Omega$ is the $\sigma$-field $\mathcal{F}$ generated by the finite-dimensional sets. For $\omega \in \Omega$ and $e \in \mathbb{E}^{d}$, the edge $e$ is called open if $\omega(e)=1$ and closed otherwise

Let $\Lambda$ be a finite box in $\mathbb{Z}^{d}$. For $b \in\{0,1\}$ define

$$
\Omega_{\Lambda}^{b}=\left\{\omega \in \Omega: \omega(e)=b \text { for } e \notin \mathbb{E}_{\Lambda}\right\},
$$

where $\mathbb{E}_{A}$ is the set of edges of $\mathbb{L}^{d}$ joining pairs of vertices belonging to $A$. On $\Omega_{\Lambda}^{b}$ we define a random-cluster measure $\phi_{\Lambda, p, q}^{b}$ as follows. Let $0 \leq p \leq 1$ and $q>0$. Let

$$
\phi_{\Lambda, p, q}^{b}(\omega)=\frac{1}{Z_{\Lambda, p, q}^{b}}\left\{\prod_{e \in \mathbb{E}_{\Lambda}} p^{\omega(e)}(1-p)^{1-\omega(e)}\right\} q^{k(\omega, \Lambda)}
$$

where $k(\omega, \Lambda)$ is the number of clusters of $\left(\mathbb{Z}^{d}, \eta(\omega)\right)$ which intersect $\Lambda$ (here, as before, $\eta(\omega)=\left\{e \in \mathbb{E}^{d}: \omega(e)=1\right\}$ is the set of open edges). The boundary condition $b=0$ (resp. $b=1)$ is sometimes termed 'free' (resp. 'wired').

Theorem 13.7. The weak limits

$$
\phi_{p, q}^{b}=\lim _{\Lambda \rightarrow \mathbb{Z}^{d}} \phi_{\Lambda, p, q}^{b}, \quad b=0,1,
$$

exist if $q \geq 1$.

Proof. Let $A$ be an increasing cylinder event (i.e., an increasing finite-dimensional event). If $\Lambda \subseteq \Lambda^{\prime}$ and $\Lambda$ includes the 'base' of $A$, then

$$
\phi_{\Lambda, p, q}^{1}(A)=\phi_{\Lambda^{\prime}, p, q}^{1}\left(A \mid \text { all edges in } \mathbb{E}_{\Lambda^{\prime} \backslash \Lambda} \text { are open }\right) \geq \phi_{\Lambda^{\prime}, p, q}^{1}(A),
$$

where we have used the tower property and the FKG inequality. Therefore the limit $\lim _{\Lambda \rightarrow \mathbb{Z}^{d}} \phi_{\Lambda, p, q}^{1}(A)$ exists by monotonicity. Since $\mathcal{F}$ is generated by such events $A$ the weak limit $\phi_{p, q}^{1}$ exists. A similar argument is valid in the case $b=0$.

The measures $\phi_{p, q}^{0}$ and $\phi_{p, q}^{1}$ are called 'random-cluster measures' on $\mathbb{L}^{d}$ with parameters $p$ and $q$. Another route to a definition of such measures uses a type of Dobrushin-Lanford-Ruelle (DLR) formalism rather than weak limits (see [163]) ${ }^{10}$ There is a set of 'DLR measures' $\phi$ satisfying $\phi_{p, q}^{0} \leq \phi \leq \phi_{p, q}^{1}$, whence there is a unique such measure if and only if $\phi_{p, q}^{0}=\phi_{p, q}^{1}$.

Henceforth we assume that $q \geq 1$. Turning to the question of phase transition, and remembering percolation, we define the percolation probabilities

$(13.8)$

$$
\theta^{b}(p, q)=\phi_{p, q}^{b}(0 \leftrightarrow \infty), \quad b=0,1,
$$

e., the probability that 0 belongs to an infinite open cluster. The corresponding critical probabilities are given by

$$
p_{\mathrm{c}}^{b}(q)=\sup \left\{p: \theta^{b}(p, q)=0\right\}, \quad b=0,1 .
$$

Faced possibly with two (or more) distinct critical probabilities, we present the following result, abstracted from $[17,159,160,163]$.

${ }^{10}$ Let $\phi_{\Lambda, p, q}^{\xi}$ be the random-cluster measure on $\Lambda$ having boundary conditions inherited from the configuration $\xi$ off $\Lambda$. It is proved in [163] that any limit point $\phi$ of the family of probability measures $\left\{\phi^{\xi} \quad: \Lambda \subseteq \mathbb{Z}^{d}, \xi \in \Omega\right\}$ is a DLR measure whenever $\phi$ has the property that the number $I$ of infinite open clusters satisfies $\phi(I \leq 1)=1$. It is an open problem to decide exactly which weak limits are DLR measures (if not all). 
Theorem 13.9. Assume that $d \geq 2$ and $q \geq 1$. There exists a countable subset $\mathcal{P}=\mathcal{P}_{q, d}$ of $[0,1]$, possibly empty, such that $\phi_{p, q}^{0}=\phi_{p, q}^{1}$ if either $\theta^{1}(p, q)=0$ or $p \notin \mathcal{P}$

Consequently, $\theta^{0}(p, q)=\theta^{1}(p, q)$ if $p$ does not belong to the countable set $\mathcal{P}_{q, d}$, whence $p_{\mathrm{c}}^{0}(q)=p_{\mathrm{c}}^{1}(q)$. Henceforth we refer to the critical value as $p_{\mathrm{c}}(q)$. It is believed that $\mathcal{P}_{q, d}=\varnothing$ for small $q$ (depending on the value of $d$ ), and that $\mathcal{P}_{q, d}=\left\{p_{\mathrm{c}}(q)\right\}$ for large $q$; see the next section.

Next we prove the non-triviality of $p_{\mathrm{c}}(q)$ for $q \geq 1$ (see [17]).

Theorem 13.10. If $d \geq 2$ and $q \geq 1$ then $0<p_{\mathrm{c}}(q)<1$.

Proof. We compare the case of general $q$ with the case $q=1$ (percolation). Using the comparison inequalities (Theorem 13.2), we find that

$\quad p_{\mathrm{c}}(1) \leq p_{\mathrm{c}}(q) \leq \frac{q p_{\mathrm{c}}(1)}{1+(q-1) p_{\mathrm{c}}(1)}, \quad q \geq 1$,

where $p_{\mathrm{c}}(1)$ is the critical probability of bond percolation on $\mathbb{L}^{d}$. Cf. Theorem 3.2 .

We note that $p_{\mathrm{c}}(q)$ is monotone non-decreasing in $q$, by use of the comparison inequalities. Actually it is strictly monotone and Lipschitz continuous (see [162]).

Finally we return to the Potts model, and we review the correspondence of phase transitions. The relevant 'order parameter' of the Potts model is given by

$$
M(\beta J, q)=\lim _{\Lambda \rightarrow \mathbb{Z}^{d}}\left\{\pi_{\Lambda, \beta, J}^{1}(\sigma(0)=1)-q^{-1}\right\},
$$

where $\pi_{\Lambda, \beta, J}^{1}$ is a Potts measure on $\Lambda$ 'with boundary condition 1'. We may think of $M(\beta J, q)$ as a measure of the degree to which the boundary condition ' 1 ' is noticed at the origin. By an application of Theorem 12.8 to a suitable graph obtained from $\Lambda$, we have that

$$
\pi_{\Lambda, \beta, J}^{1}(\sigma(0)=1)-q^{-1}=\left(1-q^{-1}\right) \phi_{\Lambda, p, q}^{1}(0 \leftrightarrow \partial \Lambda)
$$

where $p=1-e^{-\beta J}$. Therefore

$$
M(\beta J, q)=\left(1-q^{-1}\right) \lim _{\Lambda \rightarrow \mathbb{Z}^{d}} \phi_{\Lambda, p, q}^{1}(0 \leftrightarrow \partial \Lambda) .
$$

By an interchange of limits (which may be justified, see $[17,163]$ ), we have that ${ }^{11}$

$$
\lim _{\Lambda \rightarrow \mathbb{Z}^{d}} \phi_{\Lambda, p, q}^{1}(0 \leftrightarrow \partial \Lambda)=\theta^{1}(p, q),
$$

whence $M(\beta J, q)$ and $\theta^{1}(p, q)$ differ only by the factor $\left(1-q^{-1}\right)$. ${ }^{11}$ We note that the corresponding limit for the free measure, $\lim _{\Lambda \rightarrow \mathbb{Z}^{d}} \phi_{\Lambda, p, q}^{0}(0 \leftrightarrow \partial \Lambda)=$
$\theta^{0}(p, q)$, has not been proved in its full generality; see [163, 316].
13.3 First and Second Order Transitions

Let $q \geq 1$ and $0 \leq p \leq 1$. As before, $\phi_{p, q}^{b}$ is the random-cluster measure on $\mathbb{L}^{d}$ constructed according to the boundary condition $b \in\{0,1\}$. The corresponding percolation probability is $\theta^{b}(p, q)=\phi_{p, q}^{b}(0 \leftrightarrow \infty)$. There is a phase transition at the point $p_{\mathrm{c}}=p_{\mathrm{c}}(q)$. Much of the interest in Potts models (and therefore randomcluster models) has been directed at a dichotomy in the type of phase transition, which depends apparently on whether $q$ is small or large. The following picture is credible but proved only in part.

(a) Small $q$, say $1 \leq q<Q(d)$. It is believed that $\phi_{p, q}^{0}=\phi_{p, q}^{1}$ for all $p$, and that $\theta^{b}\left(p_{\mathrm{c}}(q), q\right)=0$ for $b=0,1$. This will imply (see [163]) that there is a unique random-cluster measure, and that each $\theta^{b}(\cdot, q)$ is continuous at the critical point. Such a transition is sometimes termed 'second order'. The two-point connectivity function

$$
\tau_{p, q}^{b}(x, y)=\phi_{p, q}^{b}(x \leftrightarrow y)
$$

satisfies

$$
-\frac{1}{n} \log \tau_{p, q}^{b}\left(0, n e_{1}\right) \rightarrow \sigma(p, q) \quad \text { as } n \rightarrow \infty
$$

where $\sigma(p, q)>0$ if and only if $p<p_{\mathrm{c}}(q)$. In particular $\sigma\left(p_{\mathrm{c}}(q), q\right)=0$.

(b) Large $q$, say $q>Q(d)$. We have that $\phi_{p, q}^{0}=\phi_{p, q}^{1}$ if and only if $p \neq p_{\mathrm{c}}(q)$. When $p=p_{\mathrm{c}}(q)$, then $\phi_{p, q}^{0}$ and $\phi_{p, q}^{1}$ are the unique translation-invariant random-cluster measures on $\mathbb{L}^{d}$. Furthermore $\theta^{0}\left(p_{\mathrm{c}}(q), q\right)=0$ and $\theta^{1}\left(p_{\mathrm{c}}(q), q\right)>0$, which implies that $\theta^{1}(\cdot, q)$ is discontinuous at the critical point. Such a transition is sometimes termed 'first order'. The limit function $\sigma$, given by (13.13) with $b=0$, satisfies $\sigma\left(p_{\mathrm{c}}(q), q\right)>0$, which is to say that the measure $\phi_{p, q}^{0}$ has exponentially decaying connectivities even at the critical point. Trivially $\sigma(p, q)=0$ when $p>p_{\mathrm{c}}(q)$, and this discontinuity at $p_{\mathrm{c}}(q)$ is termed the 'mass gap'.

It is further believed that $Q(d)$ is non-increasing in $d$ with

$$
Q(d)= \begin{cases}4 & \text { if } d=2 \\ 2 & \text { if } d \geq 6 .\end{cases}
$$

Some progress has been made towards verifying the main features of this picture. When $d=2$, special properties of two-dimensional space (particularly, a duality property) may be utilised (see Section 13.5). As for general values of $d$, we have partial information when $q=1, q=2$, or $q$ is sufficiently large. There is no full proof of a 'sharp cut-off' in the value of $q$, i.e., the existence of a critical value $Q(d)$ for $q$ (even when $d=2$, but see [191]).

Specifically, the following is known.

(c) When $q=1$, it is elementary that there is a unique random-cluster measure, namely product measure. Also, $\theta\left(p_{\mathrm{c}}(1), 1\right)=0$ if $d=2$ or $d \geq 19$ (and perhaps for other $d$ also). There is no mass gap, but $\sigma(p, q)>0$ for $p<p_{\mathrm{c}}(1)$. See [G]. 
(d) When $q=2$, we have information via technology developed for the Ising model. For example, $\theta^{1}\left(p_{\mathrm{c}}(2), 2\right)=0$ if $d \neq 3$. Also, $\sigma(p, 2)>0$ if $p<p_{\mathrm{c}}(2)$. See [14].

(e) When $q$ is sufficiently large, the Pirogov-Sinai theory of contours may be applied to obtain the picture described in (b) above. See [223, 225, 226, 255].

Further information about the above arguments is presented in Section 13.5 for the special case of two dimensions.

13.4 Exponential Decay in the Subcritical Phase

The key theorem for understanding the subcritical phase of percolation states that long-range connections have exponentially decaying probabilities (Theorem 6.10) Such a result is believed to hold for all random-cluster models with $q \geq 1$, but no full proof has been found. The result is known only when $q=1, q=2$, or $q$ is sufficiently large, and the three sets of arguments for these cases are somewhat different from one another. As for results valid for all $q(\geq 1)$, the best that is currently known is that the connectivity function decays exponentially whenever it decays at a sufficient polynomial rate. We describe this result in this section; see [168] for more details.

As a preliminary we introduce another definition of a critical point. Let

(13.15) $\quad Y(p, q)=\limsup _{n \rightarrow \infty}\left\{n^{d-1} \phi_{p, q}^{0}(0 \leftrightarrow \partial B(n))\right\}$

and

$$
p_{\mathrm{g}}(q)=\sup \{p: Y(p, q)<\infty\}
$$

$(13.16)$

Evidently $p_{\mathrm{g}}(q) \leq p_{\mathrm{c}}(q)$, and it is believed that equality is valid here. Next, we define the $k$ th iterate of (natural) logarithm by

$$
\lambda_{1}(n)=\log n, \quad \lambda_{k}(n)=\log ^{+}\left\{\lambda_{k-1}(n)\right\} \quad \text { for } k \geq 2
$$

where $\log ^{+} x=\max \{1, \log x\}$.

We present the next theorem in two parts, and shall give a full proof of part (a) only; for part (b), see [168].

Theorem 13.17. Let $0<p<1$ and $q \geq 1$, and assume that $p<p_{\mathrm{g}}(q)$.

(a) If $k \geq 1$, there exists $\alpha=\alpha(p, q, k)$ satisfying $\alpha>0$ such that

$$
\phi_{p, q}^{0}(0 \leftrightarrow \partial B(n)) \leq \exp \left\{-\alpha n / \lambda_{k}(n)\right\} \quad \text { for all large } n .
$$

(b) If (13.18) holds, then there exists $\beta=\beta(p, q)$ satisfying $\beta>0$ such that

$$
\phi_{p, q}^{0}(0 \leftrightarrow \partial B(n)) \leq e^{-\beta n} \quad \text { for all large } n .
$$

The spirit of the theorem is close to that of Hammersley [174] and Simon-Lieb $[235,332]$, who derived exponential estimates when $q=1,2$, subject to a hypothesis of finite susceptibility (i.e., that $\left.\sum_{x} \phi_{p, q}^{0}(0 \leftrightarrow x)<\infty\right)$. The latter hypothesis is slightly stronger than the assumption of Theorem 13.17 when $d=2$.

Underlying any theorem of this type is an inequality. In this case we use two, of which the first is a consequence of the following version of Russo's formula, taken from [75].
Theorem 13.19. Let $0<p<1, q>0$, and let $\psi_{p}$ be the corresponding randomcluster measure on a finite graph $G=(V, E)$. Then

$$
\frac{d}{d p} \psi_{p}(A)=\frac{1}{p(1-p)}\left\{\psi_{p}\left(N 1_{A}\right)-\psi_{p}(N) \psi_{p}(A)\right\}
$$

for any event $A$, where $N=N(\omega)$ is the number of open edges of a configuration $\omega$. Here, $\psi_{p}$ is used both as probability measure and expectation operator.

Proof. We express $\psi_{p}(A)$ as

$$
\psi_{p}(A)=\frac{\sum_{\omega} 1_{A}(\omega) \pi_{p}(\omega)}{\sum_{\omega} \pi_{p}(\omega)}
$$

where $\pi_{p}(\omega)=p^{N(\omega)}(1-p)^{|E|-N(\omega)} q^{k(\omega)}$. Now differentiate throughout with respect to $p$, and gather the terms to obtain the required formula.

Lemma 13.20. Let $0<p<1$ and $q \geq 1$. For any non-empty increasing event $A$,

$$
\frac{d}{d p}\left\{\log \psi_{p}(A)\right\} \geq \frac{\psi_{p}\left(F_{A}\right)}{p(1-p)}
$$

where

$$
F_{A}(\omega)=\inf \left\{\sum_{e}\left(\omega^{\prime}(e)-\omega(e)\right): \omega^{\prime} \geq \omega, \omega^{\prime} \in A\right\}
$$

Proof. It may be checked that $F_{A} 1_{A}=0$, and that $N+F_{A}$ is increasing. Therefore, by the FKG inequality,

$$
\begin{aligned}
\psi_{p}\left(N 1_{A}\right)-\psi_{p}(N) \psi_{p}(A) & =\psi_{p}\left(\left(N+F_{A}\right) 1_{A}\right)-\psi_{p}(N) \psi_{p}(A) \\
& \geq \psi_{p}\left(F_{A}\right) \psi_{p}(A) .
\end{aligned}
$$

Now use Theorem 13.19

The quantity $F_{A}$ is central to the proof of Theorem 13.17 . In the proof, we shall make use of the following fact. If $A$ is increasing and $A \subset B_{1} \cap B_{2} \cap \cdots \cap B_{m}$, where the $B_{i}$ are cylinder events defined on disjoint sets of edges, then

$$
F_{A} \geq \sum_{i=1}^{m} F_{B_{i}}
$$


Lemma 13.22. Let $q \geq 1$ and $0<r<s<1$. There exists a function $c=c(r, s, q)$, satisfying $1<c<\infty$, such that

$$
\psi_{r}\left(F_{A} \leq k\right) \leq c^{k} \psi_{s}(A) \quad \text { for all } k \geq 0
$$

and for all increasing events $A$.

Proof. We sketch this, which is similar to the so called 'sprinkling lemma' of [15]; see also $[\mathrm{G}, 168]$.

Let $r<s$. The measures $\psi_{r}$ and $\psi_{s}$ may be coupled together in a natural way. That is, there exists a probability measure $\mu$ on $\Omega_{E}^{2}=\{0,1\}^{E} \times\{0,1\}^{E}$ such that:

(a) the first marginal of $\mu$ is $\psi_{r}$,

b) the second marginal of $\mu$ is $\psi_{s}$

(c) $\mu$ puts measure 1 on the set of configurations $(\pi, \omega) \in \Omega_{E}^{2}$ such that $\pi \leq \omega$. Furthermore $\mu$ may be found such that the following holds. There exists a positive number $\beta=\beta(r, s, q)$ such that, for any fixed $\xi \in \Omega_{E}$ and subset $B$ of edges (possibly depending on $\xi$ ), we have that

$$
\frac{\mu(\{(\pi, \omega): \omega(e)=1 \text { for } e \in B, \pi=\xi\})}{\mu(\{(\pi, \omega): \pi=\xi\})} \geq \beta^{|B|} .
$$

That is to say, conditional on the first component of a pair $(\pi, \omega)$ sampled according to $\mu$, the measure of the second component dominates a non-trivial product measure. Now suppose that $\xi\left(\in \Omega_{E}\right)$ is such that $F_{A}(\xi) \leq k$, and find a set $B=B(\xi)$ of edges, such that $|B| \leq k$, and with the property that $\xi^{B} \in A$, where $\xi^{B}$ is the configuration obtained from $\xi$ by declaring all edges in $B$ to be open. By (13.23),

$$
\begin{aligned}
\psi_{s}(A) & \geq \sum_{\xi: F_{A}(\xi) \leq k} \mu(\{(\pi, \omega): \omega(e)=1 \text { for } e \in B, \pi=\xi\}) \\
& \geq \beta^{k} \psi_{r}\left(F_{A} \leq k\right)
\end{aligned}
$$

as required.

Proof of Theorem 13.17. (a) Write $A_{n}=\{0 \leftrightarrow \partial B(n)\}$ and $\psi_{p}=\phi_{B(m), p, q}^{0}$ where $p<p_{\mathrm{c}}=p_{\mathrm{c}}(q)$. We apply Lemma 13.20 (in an integrated form), and pass to the limit as $m \rightarrow \infty$, to obtain that the measures $\phi_{p, q}=\phi_{p, q}^{0}$ satisfy

$$
\phi_{r, q}\left(A_{n}\right) \leq \phi_{s, q}\left(A_{n}\right) \exp \left\{-4(s-r) \phi_{s, q}\left(F_{n}\right)\right\}, \quad \text { if } r \leq s,
$$

where $F_{n}=F_{A_{n}}$ (we have used Theorem 13.2(a) here, together with the fact that $F_{n}$ is a decreasing random variable).

Similarly, by summing the corresponding inequality of Lemma 13.22 over $k$, and letting $m \rightarrow \infty$, we find that

$$
\phi_{r, q}\left(F_{n}\right) \geq \frac{-\log \phi_{s, q}\left(A_{n}\right)}{\log c}-\frac{c}{c-1} \quad \text { if } r<s .
$$

We shall use (13.24) and (13.25) in an iterative scheme. At the first stage, assume $r<s<t<p_{\mathrm{g}}=p_{\mathrm{g}}(q)$. Find $c_{1}(t)$ such that

$(13.26)$

$$
\phi_{p, q}\left(A_{n}\right) \leq \frac{c_{1}(t)}{n^{d-1}} \quad \text { for all } n
$$

By (13.25),

$$
\phi_{s, q}\left(F_{n}\right) \geq \frac{(d-1) \log n}{\log c}+\mathrm{O}(1),
$$

which we insert into (13.24) to obtain

$$
\phi_{r, q}\left(A_{n}\right) \leq \frac{c_{2}(r)}{n^{d-1+\Delta_{2}(r)}} \quad \text { for all } n,
$$

and for some constants $c_{2}(r), \Delta_{2}(r)(>0)$. This is an improvement over (13.26).

At the next stages we shall need to work slightly harder. Fix a positive integer $m$, and let $R_{i}=i m$ for $0 \leq i \leq K$ where $K=\lfloor n / m\rfloor$. Let $L_{i}=\left\{\partial B\left(R_{i}\right) \leftrightarrow \partial B\left(R_{i+1}\right)\right\}$ and $H_{i}=F_{L_{i}}$. By $(13.21)$,

$$
F_{n} \geq \sum_{i=0}^{K-1} H_{i}
$$

Now there exists a constant $\eta(<\infty)$ such that

$$
\phi_{p, q}\left(L_{i}\right) \leq\left|\partial B\left(R_{i}\right)\right| \phi_{p, q}\left(A_{m}\right) \leq \eta n^{d-1} \phi_{p, q}\left(A_{m}\right)
$$

for $0 \leq i \leq K-1$.

Let $r<s<p_{\mathrm{g}}$, and let $c_{2}=c_{2}(s), \Delta_{2}=\Delta_{2}(s)$ as in (13.27). From (13.28)$(13.29)$

$$
\begin{aligned}
\phi_{s, q}\left(F_{n}\right) & \geq \sum_{i=0}^{K-1} \phi_{s, q}\left(\overline{L_{i}}\right) \geq K\left(1-\eta n^{d-1} \phi_{p, q}\left(A_{m}\right)\right) \\
& \geq K\left(1-\eta n^{d-1} \frac{c_{2}}{m^{d-1+\Delta_{2}}}\right) .
\end{aligned}
$$

We now choose $m$ by

$$
m=\left\{\left(2 \eta c_{2}\right) n^{d-1}\right\}^{1 /\left(d-1+\Delta_{2}\right)}
$$

(actually, an integer close to this value) to find that

$$
\phi_{s, q}\left(F_{n}\right) \geq \frac{1}{2} K \geq D n^{\Delta_{3}}
$$

for some $D>0,0<\Delta_{3}<1$. Substitute into (13.24) to obtain

$$
\phi_{r, q}\left(A_{n}\right) \leq \exp \left\{-c_{3} n^{\Delta_{3}}\right\}
$$

for some positive $c_{3}=c_{3}(r), \Delta_{3}=\Delta_{3}(r)$. This improves (13.27) substantially. 
We repeat the last step, using (13.30) in place of (13.27), to obtain

$(13.31)$

$$
\phi_{r, q}\left(A_{n}\right) \leq \exp \left\{-\frac{c_{4} n}{(\log n)^{\Delta_{4}}}\right\} \quad \text { if } r<p_{\mathrm{g}}
$$

for some $c_{4}=c_{4}(r)>0$ and $1<\Delta_{4}=\Delta_{4}(r)<\infty$.

At the next stage, we use (13.28)-(13.29) more carefully. This time, set $m=$ $(\log n)^{2}$, and let $r<s<t<p_{\mathrm{g}}$. By (13.29) and (13.31),

$$
\phi_{t, q}\left(L_{i}\right) \leq \eta n^{d-1} \exp \left\{-\frac{c_{4} m}{(\log m)^{\Delta_{4}}}\right\}
$$

which, via Lemma 13.22 , implies as in (13.25) that

$$
\phi_{s, q}\left(H_{i}\right) \geq \frac{D(\log n)^{2}}{(\log \log n)^{\Delta_{4}}}
$$

for some $D>0$. By (13.28), and the fact that $K=\lfloor n / m\rfloor$,

$$
\phi_{s, q}\left(F_{n}\right) \geq \frac{D^{\prime} n}{(\log \log n)^{\Delta_{4}}}
$$

for some $D^{\prime}>0$. By (13.24),

$$
\phi_{r, q}\left(A_{n}\right) \leq \exp \left\{-\frac{c_{5} n}{(\log \log n)^{\Delta_{4}}}\right\}
$$

Since $\Delta_{4}>1$, this implies the claim of the theorem with $k=1$. The claim for general $k$ requires $k-1$ further iterations of the argument.

(b) We omit the proof of this part. The fundamental argument is taken from [139] and the details are presented in [168].

13.5 The Case of Two Dimensions

In this section we consider the case of random-cluster measures on the square lattice $\mathbb{L}^{2}$. Such measures have a property of self-duality which generalises that of bond percolation. We begin by describing this duality.

Let $G=(V, E)$ be a plane graph with planar dual $G^{\mathrm{d}}=\left(V^{\mathrm{d}}, E^{\mathrm{d}}\right)$. Any configuration $\omega \in \Omega_{E}$ gives rise to a dual configuration $\omega^{\mathrm{d}} \in \Omega_{E^{\mathrm{d}}}$ defined as follows. If $e$ $(\in E)$ is crossed by the dual edge $e^{\mathrm{d}}\left(\in E^{\mathrm{d}}\right)$, we define $\omega^{\mathrm{d}}\left(e^{\mathrm{d}}\right)=1-\omega(e)$. As usual, $\eta(\omega)$ denotes the set $\{e: \omega(e)=1\}$ of edges which are open in $\omega$. By drawing a picture, one may be convinced that every face of $(V, \eta(\omega))$ contains a unique component of $\left(V^{\mathrm{d}}, \eta\left(\omega^{\mathrm{d}}\right)\right)$, and therefore the number $f(\omega)$ of faces (including the infinite face) of $(V, \eta(\omega))$ satisfies $f(\omega)=k\left(\omega^{\mathrm{d}}\right)$. See Figure 13.1. (Note that this definition

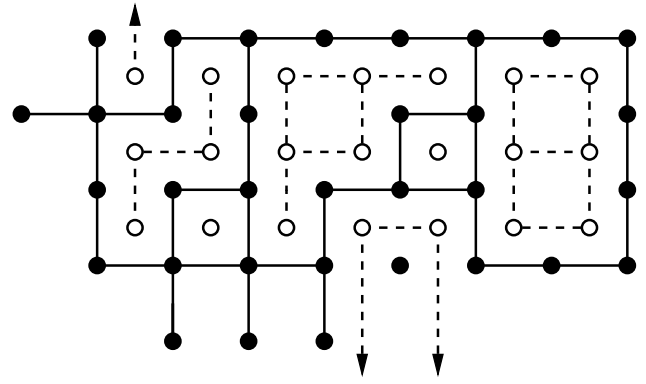

Fig. 13.1. A primal configuration $\omega$ (with solid lines) and its dual configuration $\omega^{\mathrm{d}}$ (with dashed lines). The arrows join the given vertices of the dual to a dual vertex in the infinite face. Note that each face of the primal graph (including the infinite face) contains a unique component of the dual graph.

of the dual configuration differs slightly from that used earlier for two-dimensional percolation.)

The random-cluster measure on $G$ is given by

$$
\phi_{G, p, q}(\omega) \propto\left(\frac{p}{1-p}\right)^{|\eta(\omega)|} q^{k(\omega)} .
$$

Using Euler's formula,

$$
k(\omega)=|V|-|\eta(\omega)|+f(\omega)-1,
$$

and the facts that $f(\omega)=k\left(\omega^{\mathrm{d}}\right)$ and $|\eta(\omega)|+\left|\eta\left(\omega^{\mathrm{d}}\right)\right|=|E|$, we have that

$$
\phi_{G, p, q}(\omega) \propto\left(\frac{q(1-p)}{p}\right)^{\left|\eta\left(\omega^{\mathrm{d}}\right)\right|} q^{k\left(\omega^{\mathrm{d}}\right)},
$$

which is to say that

$$
\phi_{G, p, q}(\omega)=\phi_{G^{\mathrm{d}}, p^{\mathrm{d}}, q}\left(\omega^{\mathrm{d}}\right) \quad \text { for } \omega \in \Omega_{E},
$$

where the dual parameter $p^{\mathrm{d}}$ is given according to

$$
\frac{p^{\mathrm{d}}}{1-p^{\mathrm{d}}}=\frac{q(1-p)}{p}
$$

The unique fixed point of the mapping $p \mapsto p^{\mathrm{d}}$ is easily seen to be given by $p=\kappa_{d}$

$$
\kappa_{q}=\frac{\sqrt{q}}{1+\sqrt{q}} .
$$


If we keep track of the constants of proportionality in the above calculation, we find that the partition function

$$
Z_{G, p, q}=\sum_{\omega \in \Omega_{E}} p^{|\eta(\omega)|}(1-p)^{|E \backslash \eta(\omega)|} q^{k(\omega)}
$$

satisfies the duality relation

$$
Z_{G, p, q}=q^{|V|-1}\left(\frac{1-p}{p^{\mathrm{d}}}\right)^{|E|} Z_{G^{\mathrm{d}}, p^{\mathrm{d}}, q}
$$

which, when $p=p^{\mathrm{d}}=\kappa_{q}$, becomes

$(13.35)$

$$
Z_{G, \kappa_{q}, q}=q^{|V|-1-\frac{1}{2}|E|} Z_{G^{\mathrm{d}}, \kappa_{q}, q} .
$$

We shall find a use for this later.

Turning to the square lattice, let $\Lambda_{n}=[0, n]^{2}$, whose dual graph $\Lambda_{n}^{\mathrm{d}}$ may be obtained from $[-1, n]^{2}+\left(\frac{1}{2}, \frac{1}{2}\right)$ by identifying all boundary vertices. This implies by (13.32) that

$$
\phi_{\Lambda_{n}, p, q}^{0}(\omega)=\phi_{\Lambda_{n}^{\mathrm{d}}, p^{\mathrm{d}}, q}^{1}\left(\omega^{\mathrm{d}}\right)
$$

for configurations $\omega$ on $\Lambda_{n}$ (and with a small 'fix' on the boundary of $\Lambda_{n}^{\mathrm{d}}$ ). Letting $n \rightarrow \infty$, we obtain that

$$
\phi_{p, q}^{0}(A)=\phi_{p^{\mathrm{d}}, q}^{1}\left(A^{\mathrm{d}}\right)
$$

for all cylinder events $A$, where $A^{\mathrm{d}}=\left\{\omega^{\mathrm{d}}: \omega \in A\right\}$.

As a consequence of this duality, we may obtain as in the proof of Theorem 9.1

$$
\theta^{0}\left(\kappa_{q}, q\right)=0
$$

(see $[163,346])$, whence the critical value of the square lattice satisfies

$$
p_{\mathrm{c}}(q) \geq \frac{\sqrt{q}}{1+\sqrt{q}} \quad \text { for } q \geq 1 .
$$

It is widely believed that

$$
p_{\mathrm{c}}(q)=\frac{\sqrt{q}}{1+\sqrt{q}} \text { for } q \geq 1 .
$$

This is known to hold when $q=1$ (percolation), when $q=2$ (Ising model), and for sufficiently large values of $q$. Following the route of the proof of Theorem 9.1, it suffices to show that

$$
\phi_{p, q}^{0}(0 \leftrightarrow \partial B(n)) \leq e^{-n \psi(p, q)} \quad \text { for all } n,
$$

and for some $\psi(p, q)$ satisfying $\psi(p, q)>0$ when $p<p_{\mathrm{c}}(q)$. (Actually, rather less than exponential decay is required; it would be enough to have decay at rate $n^{-1}$.) This was proved by the work of $[14,235,332]$ when $q=2$. When $q$ is large, this and more is known. Let $\mu$ be the connective constant of $\mathbb{L}^{2}$, and let $Q=\left\{\frac{1}{2}\left(\mu+\sqrt{\mu^{2}-4}\right)\right\}^{4}$. We have that $2.620<\mu<2.696$ (see [335]), whence $21.61<Q<25.72$. We set

$$
\psi(q)=\frac{1}{24} \log \left\{\frac{(1+\sqrt{q})^{4}}{q \mu^{4}}\right\},
$$

noting that $\psi(q)>0$ if and only if $q>Q$.

Theorem 13.40. If $d=2$ and $q>Q$ then the following hold.

a) The critical point is given by $p_{\mathrm{c}}(q)=\sqrt{q} /(1+\sqrt{q})$.

(b) We have that $\theta^{1}\left(p_{\mathrm{c}}(q), q\right)>0$.

(c) For any $\psi<\psi(q)$

$$
\phi_{p_{\mathrm{c}}(q), q}^{0}(0 \leftrightarrow \partial B(n)) \leq e^{-n \psi} \quad \text { for all large } n .
$$

We stress that these conclusions may be obtained for general $d(\geq 2)$ when $q$ is sufficiently large $(q>Q=Q(d))$, as may be shown using so called Pirogov-Sinai theory (see [225]). In the case $d=2$ presented here, the above duality provides a beautiful and simple proof. This proof is an adaptation and extension of that of $[226]$

Proof. Let $B=B(n)=[-n, n]^{2}$ as usual, and let $B^{\mathrm{d}}=[-n, n-1]^{2}+\left(\frac{1}{2}, \frac{1}{2}\right)$ be those vertices of the dual of $B(n)$ which lie inside $B(n)$ (i.e., we omit the vertex in the infinite face of $B$ ). We shall work with 'wired' boundary conditions on $B$, and we let $\omega$ be a configuration on the edges of $B$. A circuit $\Gamma$ of $B^{\text {d }}$ is called an outer circuit of a configuration $\omega$ if the following properties hold:

(a) all edges of $\Gamma$ are open in the dual configuration $\omega^{\mathrm{d}}$, which is to say that they traverse closed edges of $B$

(b) the origin of $\mathbb{L}^{2}$ is in the interior of $\Gamma$,

(c) every vertex of $B$ lying in the exterior of $\Gamma$, but within distance of $1 / \sqrt{2}$ of some vertex of $\Gamma$, belongs to the same component of $\omega$.

See Figure 13.2 for an illustration of the meaning of 'outer circuit'.

Each circuit $\Gamma$ of $B^{\text {d }}$ partitions the set $\mathbb{E}_{B}$ of edges of $B$ into three sets, being

$$
\begin{aligned}
E & =\{\text { edges of } B \text { exterior to } \Gamma\}, \\
I & =\{\text { edges of } B \text { interior to } \Gamma\}, \\
\Gamma^{\prime} & =\{\text { edges of } B \text { crossing } \Gamma\} .
\end{aligned}
$$

The edges $I$ form a connected subgraph of $B$

Our target is to obtain an upper bound for the probability that a given $\Gamma$ is an outer circuit. This we shall do by examining certain partition functions. Since no open component of $\omega$ contains points lying in both the exterior and interior of 


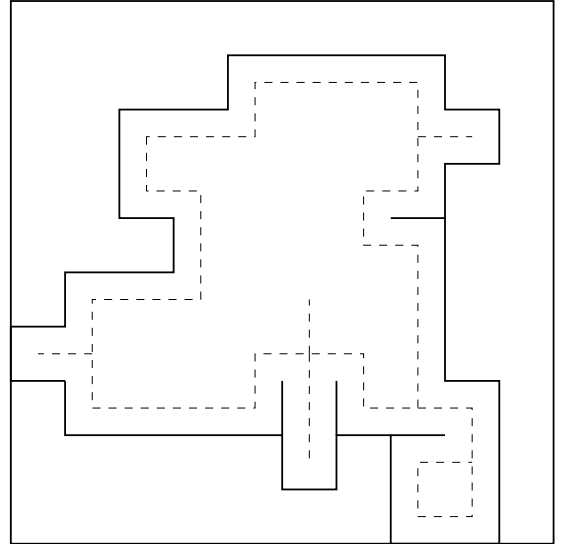

Fig. 13.2. The dashed lines include an outer circuit $\Gamma$ of the dual $B^{\mathrm{d}}$

an outer circuit, the event $\mathrm{OC}(\Gamma)=\{\Gamma$ is an outer circuit $\}$ satisfies, for any dual circuit $\Gamma$ having 0 in its interior,

$(13.41)$

$$
\begin{aligned}
\phi_{B, p, q}^{1}(\mathrm{OC}(\Gamma)) & =\frac{1}{Z_{B, p, q}^{1}} \sum_{\omega} 1_{\mathrm{OC}(\Gamma)}(\omega) \pi_{p}(\omega) \\
& =\frac{1}{Z_{B, p, q}^{1}}(1-p)^{|\Gamma|} Z_{E}^{1}(\Gamma) Z_{I}
\end{aligned}
$$

where $\pi_{p}(\omega)=p^{N(\omega)}(1-p)^{|E|-N(\omega)} q^{k(\omega)}, Z_{E}^{1}(\Gamma)$ is the sum of $\pi_{p}\left(\omega^{\prime}\right)$ over all $\omega^{\prime} \in$ $\{0,1\}^{E}$ with ' 1 ' boundary conditions on $\partial B$ and consistent with $\Gamma$ being an outer circuit (i.e., property (c) above), and $Z_{I}$ is the sum of $\pi_{p}\left(\omega^{\prime \prime}\right)$ over all $\omega^{\prime \prime} \in\{0,1\}^{I}$.

Next we use duality. Let $I^{\mathrm{d}}$ be the set of dual edges which cross the primal edges $I$, and let $m$ be the number of vertices of $B$ inside $\Gamma$. By (13.34),

$(13.42)$

$$
Z_{I}=q^{m-1}\left(\frac{1-p}{p^{\mathrm{d}}}\right)^{|I|} Z_{I^{\mathrm{d}}, p^{\mathrm{d}}, q}^{1}
$$

where $p^{\mathrm{d}}$ satisfies (13.33), and where $Z_{I^{\mathrm{d}}, p^{\mathrm{d}}, q}^{1}$ is the partition function for dual configurations, having wired boundary conditions, on the set $V^{\mathrm{d}}$ of vertices incident to $I^{\mathrm{d}}$ (i.e., all vertices of $V^{\mathrm{d}}$ on its boundary are identified, as indicated in Figure $13.3)$

We note two general facts about partition functions. First, for any graph $G$ $Z_{G, p, q} \geq 1$ if $q \geq 1$. Secondly, $Z \cdot, p, q$ has a property of supermultiplicativity when $q \geq 1$, which implies in particular that

$$
Z_{B, p, q}^{1} \geq Z_{E}^{1}(\Gamma) Z_{I \cup \Gamma^{\prime}, p, q}^{1}
$$

for any circuit $\Gamma$ of $B^{\mathrm{d}}$. (This is where we use property (c) above.)
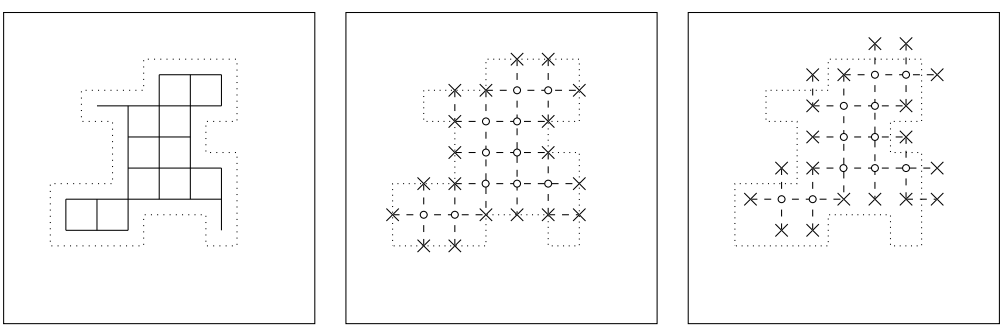

Fig. 13.3. The interior edges $I$ of $\Gamma$ are marked in the leftmost picture, and the dual $I^{\mathrm{d}}$ in the centre picture (the vertices marked with a cross are identified as a single vertex). The shifted set $I^{*}=I^{\mathrm{d}}+\left(\frac{1}{2}, \frac{1}{2}\right)$ is drawn in the rightmost picture. Note

Let $I^{*}=I^{\mathrm{d}}+\left(\frac{1}{2}, \frac{1}{2}\right)$, where $I^{\mathrm{d}}$ is thought of as a subset of $\mathbb{R}^{2}$. Note from Figure 13.3 that $I^{*} \subseteq I \cup \Gamma^{\prime}$. Using the two general facts above, we have that

$$
Z_{B, p, q}^{1} \geq Z_{E}^{1}(\Gamma) Z_{I^{*}, p, q}^{1}=Z_{E}^{1}(\Gamma) Z_{I^{\mathrm{d}}, p, q}^{1} .
$$

Now assume that $p=\sqrt{q} /(1+\sqrt{q})$, so that $p=p^{\mathrm{d}}$. Then, by (13.41)-(13.43) and (13.35),

$$
\begin{aligned}
\phi_{B, p, q}^{1}(\mathrm{OC}(\Gamma)) & =(1-p)^{|\Gamma|} \frac{Z_{E}^{1}(\Gamma) Z_{I}}{Z_{B, p, q}^{1}} \\
& =(1-p)^{|\Gamma|} q^{m-1-\frac{1}{2}|I|} \frac{Z_{E}^{1}(\Gamma) Z_{I^{\mathrm{d}}, p, q}^{1}}{Z_{B, p, q}^{1}} \\
& \leq(1-p)^{|\Gamma|} q^{m-1-\frac{1}{2}|I|} .
\end{aligned}
$$

Since each vertex of $B$ (inside $\Gamma$ ) has degree 4 , we have that

$$
4 m=2|I|+|\Gamma|,
$$

whence

$(13.45)$

$$
\phi_{B, p, q}^{1}(\mathrm{OC}(\Gamma)) \leq(1-p)^{|\Gamma|} q^{\frac{1}{4}|\Gamma|-1}=\frac{1}{q}\left(\frac{q}{(1+\sqrt{q})^{4}}\right)^{|\Gamma| / 4} .
$$

The number of dual circuits of $B$ having length $l$ and containing the origin in their interior is no greater than $l a_{l}$, where $a_{l}$ is the number of self-avoiding walks of $\mathbb{L}^{2}$ beginning at the origin and having length $l$. Therefore

$$
\sum_{\Gamma} \phi_{B, p, q}^{1}(\mathrm{OC}(\Gamma)) \leq \sum_{l=4}^{\infty} \frac{1}{q}\left(\frac{q}{(1+\sqrt{q})^{4}}\right)^{l / 4} l a_{l} .
$$




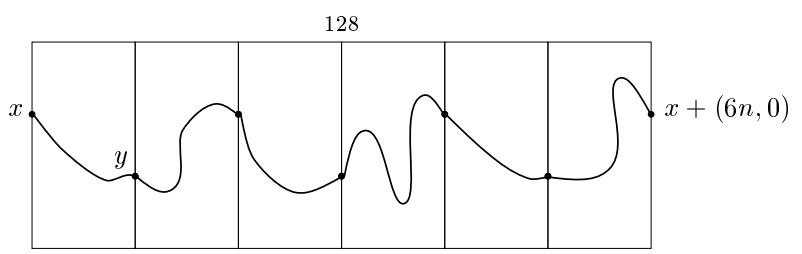

Now $l^{-1} \log a_{l} \rightarrow \mu$ as $l \rightarrow \infty$, where $\mu$ is the connective constant of $\mathbb{L}^{2}$. Suppose now that $q>Q$, so that $q \mu^{4}<(1+\sqrt{q})^{4}$. It follows that there exists $A(q)(<\infty)$ such that

$$
\sum_{\Gamma} \phi_{B, p, q}^{1}(\mathrm{OC}(\Gamma))<A(q) \text { for all } n .
$$

If $A(q)<1$ (which holds for sufficiently large $q$ ), then

$$
\begin{aligned}
\phi_{B, p, q}^{1}(0 \leftrightarrow \partial B) & =\phi_{B, p, q}^{1}(\mathrm{OC}(\Gamma) \text { occurs for no } \Gamma) \\
& \geq 1-A(q)>0 .
\end{aligned}
$$

(We have used the assumption of wired boundary conditions here.) This implies, by taking the limit $n \rightarrow \infty$, that $\theta^{1}(p, q)>0$ when $p=\sqrt{q} /(1+\sqrt{q})$. Using (13.39), this implies parts (a) and (b) of the theorem, when $q$ is sufficiently large.

For general $q>Q$, we have only that $A(q)<\infty$. In this case, we find $N(<n)$ such that

$$
\sum_{\Gamma \text { outside } B(N)} \phi_{B, p, q}^{1}(\mathrm{OC}(\Gamma))<\frac{1}{2}
$$

where $\Gamma$ is said to be outside $B(N)$ if it contains $B(N)$ in its interior. This implies that $\phi_{B, p, q}^{1}(B(N) \leftrightarrow \partial B) \geq \frac{1}{2}$. Let $n \rightarrow \infty$, and deduce that $\phi_{p, q}^{1}(B(N) \leftrightarrow \infty) \geq \frac{1}{2}$, implying that $\theta^{1}(p, q)>0$ as required.

Turning to part (c $)^{12}$, let $p=p^{\mathrm{d}}=\sqrt{q} /(1+\sqrt{q})$ and $n \leq r$. Let $A_{n}$ be the (cylinder) event that the point $\left(\frac{1}{2}, \frac{1}{2}\right)$ lies in the interior of an open circuit of length at least $n$, this circuit having the property that its interior is contained in the interior of no open circuit having length strictly less than $n$. We have from (13.36) and (13.45) that

$(13.46)$

$$
\phi_{B(r), p, q}^{0}\left(A_{n}\right) \leq \sum_{m=n}^{\infty} \frac{m a_{m}}{q}\left(\frac{q}{(1+\sqrt{q})^{4}}\right)^{m / 4} \quad \text { for all large } r .
$$

Here, we use the observation that, if $A_{n}$ occurs in $B(r)$, then there exists a maximal open circuit $\Gamma$ of $B(r)$ containing $\left(\frac{1}{2}, \frac{1}{2}\right)$. In the dual of $B(r), \Gamma$ constitutes an outer circuit.]

We write $\mathrm{LR}_{n}$ for the event that there is an open crossing of the rectangle $R_{n}=[0, n] \times[0,2 n]$ from its left to its right side, and we set $\lambda_{n}=\phi_{p, q}^{0}\left(\mathrm{LR}_{n}\right)$. We may find a point $x$ on the left side of $R_{n}$ and a point $y$ on the right side such that

$$
\phi_{p, q}^{0}\left(x \leftrightarrow y \text { in } R_{n}\right) \geq \frac{\lambda_{n}}{(2 n+1)^{2}} .
$$

By placing six of these rectangles side by side (as in Figure 13.4), we find by the FKG inequality that

(13.47) $\quad \phi_{p, q}^{0}(x \leftrightarrow x+(6 n, 0)$ in $[0,6 n] \times[0,2 n]) \geq\left(\frac{\lambda_{n}}{(2 n+1)^{2}}\right)^{6}$.

${ }^{12}$ The present proof was completed following a contribution by Ken Alexander, see [37] for related material.

Fig. 13.4. Six copies of a rectangle having width $n$ and height $2 n$ may be put together to joining the images of $x$ and $y$, then the larger rectangle is crossed between its shorter sides

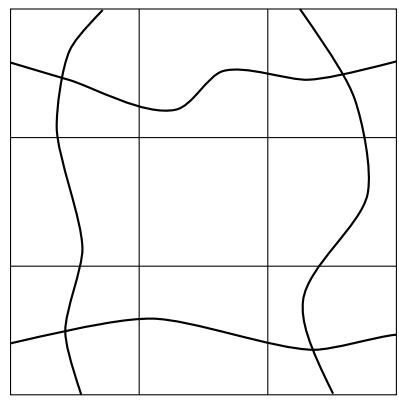

Fig. 13.5. If each of four rectangles having dimensions $6 n$ by $2 n$ is crossed by an open path between its shorter sides, then the annulus contains an open circuit having the origin in its interior

We now use four copies of the rectangle $[0,6 n] \times[0,2 n]$ to construct an annulus around the origin (see Figure 13.5). If each of these copies contains an open crossing, then the annulus contains a circuit. Using the FKG inequality again, we deduce that

$$
\phi_{p, q}^{0}\left(A_{4 n}\right) \geq\left(\frac{\lambda_{n}}{(2 n+1)^{2}}\right)^{24}
$$

Finally, if $0 \leftrightarrow \partial B(n)$, then one of the four rectangles $[0, n] \times[-n, n],[-n, n] \times$ $[0, n],[-n, 0] \times[-n, n],[-n, n] \times[-n, 0]$ is traversed by an open path betwen its two onger sides. This implies that

$$
\phi_{p, q}^{0}(0 \leftrightarrow \partial B(n)) \leq 4 \lambda_{n} .
$$

Combining (13.46)-(13.49), we obtain that

$$
\begin{aligned}
\phi_{p, q}^{0}(0 \leftrightarrow \partial B(n)) & \leq 4\left\{(2 n+1)^{2} \phi_{p, q}^{0}\left(A_{4 n}\right)\right\}^{1 / 24} \\
& \leq 4\left\{(2 n+1)^{2} \sum_{m=4 n}^{\infty} \frac{m a_{m}}{q}\left(\frac{q}{(1+\sqrt{q})^{4}}\right)^{m / 4}\right\}^{1 / 24} .
\end{aligned}
$$

As before, $m^{-1} \log a_{m} \rightarrow \mu$ as $m \rightarrow \infty$, whence part (c) follows. 


\section{References}

1. Abraham, D. B., Fontes, L., Newman, C. M., and Piza, M. S. T. (1995) Surface deconstruction and roughening in the multi-ziggurat model of wetting. Physical Review E 52, R1257-R1260.

2. Abraham, D. B. and Newman, C. M. (1988). Wetting in a three dimensional system: an exact solution. Physical Review Letters 61, 1969-1972.

3. Abraham, D. B. and Newman, C. M. (1989). Surfaces and Peierls contours: 3-d wetting and 2-d Ising percolation. Communications in Mathematical Physics 125, 181-200.

4. Abraham, D. B. and Newman, C. M. (1990). Recent exact results on wetting. Wetting Phenomena (J. De Coninck and F. Dunlop, eds.), Lecture Notes in Physics, vol. 354, Springer, Berlin, pp. 13-21.

5. Abraham, D. B. and Newman, C. M. (1991). Remarks on a random surface. Stochastic Orders and Decision under Risk (K. Mosler and M. S. Scarsini, eds.), IMS Lecture Notes, Monograph Series, vol. 19, pp. 1-6.

6. Abraham, D. B. and Newman, C. M. (1991). The wetting transition in a random surface model. Journal of Statistical Physics 63, 1097-1111.

7. Aharony, A. and Stauffer, D. (1991). Introduction to Percolation Theory Second edition). Taylor and Francis.

8. Ahlfors, L. V. (1966). Complex Analysis. McGraw-Hill, New York.

9. Aizenman, M. (1982). Geometric analysis of $\phi^{4}$ fields and Ising models. Communications in Mathematical Physics 86, 1-48.

10. Aizenman, M. (1995). The geometry of critical percolation and conformal invariance. Proceedings STATPHYS 1995 (Xianmen) (Hao Bai-lin, ed.), World Scientific

11. Aizenman, M. (1996). The critical percolation web: construction and conjectured conformal invariance properties, in preparation.

12. Aizenman, M. (1997). On the number of incipient spanning clusters. Nuclear Physics B 485, 551-582.

13. Aizenman, M. and Barsky, D. J. (1987). Sharpness of the phase transition in percolation models. Communications in Mathematical Physics 108, 489-526.

14. Aizenman, M., Barsky, D. J., and Fernández, R. (1987). The phase transitio in a general class of Ising-type models is sharp. Journal of Statistical Physics 47, 343-374

15. Aizenman, M., Chayes, J. T., Chayes, L., Fröhlich, J, and Russo, L. (1983). On a sharp transition from area law to perimeter law in a system of random surfaces. Communications in Mathematical Physics 92, 19-69.

16. Aizenman, M., Chayes, J. T., Chayes, L., and Newman, C. M. (1987). The phase boundary in dilute and random Ising and Potts ferromagnets. Journa of Physics A: Mathematical and General 20, L313.

17. Aizenman, M., Chayes, J. T., Chayes, L., and Newman, C. M. (1988). Discontinuity of the magnetization in one-dimensional $1 /|x-y|^{2}$ Ising and Potts models. Journal of Statistical Physics 50, 1-40.

18. Aizenman, M. and Fernández, R. (1986). On the critical behavior of the magnetization in high-dimensional Ising models. Journal of Statistical Physics 44, 393-454.
19. Aizenman, M. and Fernández, R. (1988). Critical exponents for long-range interactions. Letters in Mathematical Physics 16, 39

20. Aizenman, M. and Grimmett, G. R. (1991). Strict monotonicity for critical points in percolation and ferromagnetic models. Journal of Statistical Physic 63, $817-835$.

21. Aizenman, M., Kesten, H., and Newman, C. M. (1987). Uniqueness of the infinite cluster and continuity of connectivity functions for short- and longrange percolation. Communications in Mathematical Physics 111, 505-532.

22. Aizenman, M., Kesten, H., and Newman, C. M. (1987). Uniqueness of the infinite cluster and related results in percolation. Percolation Theor and Ergodic Theory of Infinite Particle Systems (H. Kesten, ed.), IMA Volumes in Mathematics and its Applications, vol. 8, Springer-Verlag, BerlinHeidelberg-New York, pp. 13-20.

23. Aizenman, M., Klein, A., and Newman, C. M. (1993). Percolation method for disordered quantum Ising models. Phase Transitions: Mathematics, Physics, Biology ... (R. Kotecky, ed.), World Scientific, Singapore, pp. 1-26.

24. Aizenman, M. and Lebowitz, J. (1988). Metastability in bootstrap percolation. Journal of Physics A: Mathematical and General 21, 3801-3813.

25. Aizenman, M., Lebowitz, J., and Bricmont, J. (1987). Percolation of the minority spins in high-dimensional Ising models. Journal of Statistical Physics 49, 859-865.

26. Aizenman, M. and Nachtergaele, B. (1994). Geometric aspects of quantum spin states. Communications in Mathematical Physics 164, 17-63.

27. Aizenman, M. and Newman, C. M. (1984). Tree graph inequalities and critical behavior in percolation models. Journal of Statistical Physics $\mathbf{3 6}$ $107-143$

28. Aldous, D. and Steele, J. M. (1992). Asymptotics for Euclidean minima spanning trees on random points. Probability Theory and Related Fields $\mathbf{9 2}$, $247-258$

29. Alexander, K. S. (1990). Lower bounds on the connectivity function in all directions for Bernoulli percolation in two and three dimensions. Annals of Probability 18, 1547-1562.

30. Alexander, K. S. (1992). Stability of the Wulff construction and fluctuations in shape for large finite clusters in two-dimensional percolation. Probability Theory and Related Fields 91, 507-532.

31. Alexander, K. S. (1993). Finite clusters in high-density continuous percolation: compression and sphericality. Probability Theory and Related Fields 97, 35-63.

32. Alexander, K. S. (1993). A note on some rates of convergence in first-passage percolation. Annals of Applied Probability 3, 81-90.

33. Alexander, K. S. (1995). Simultaneous uniqueness of infinite graphs in stationary random labeled graphs. Communications in Mathematical Physics 168, 39-55.

34. Alexander, K. S. (1995). Percolation and minimal spanning forests in infinite graphs. Annals of Probability 23, 87-104.

35. Alexander, K. S. (1996). Approximation of subadditive functions and convergence rates in limiting-shape results. Annals of Probability (to appear). 
36. Alexander, K. S. (1996). The RSW theorem for continuum percolation and the CLT for Euclidean minimal spanning trees. Annals of Applied Probability 6, 466-494.

37. Alexander, K. S. (1996). On weak mixing in lattice models (to appear).

38. Alexander, K. S., Chayes, J. T., and Chayes, L. (1990). The Wulff construction and asymptotics of the finite cluster distribution for twodimensional Bernoulli percolation. Communications in Mathematical Physics $131,1-50$.

39. Alexander, K. S. and Molchanov, S. A. (1994). Percolation of level sets for two-dimensional random fields with lattice symmetry. Journal of Statistical Physics 77, 627-643.

40. Andjel, E. (1993). Characteristic exponents for two-dimensional bootstrap percolation. Annals of Probability 21, 926-935.

41. Andjel, E. and Schinazi, R. (1996). A complete convergence theorem for an epidemic model. Journal of Applied Probability 33, 741-748.

42. Antal, P. and Pisztora, A. (1996). On the chemical distance for supercritical Bernoulli percolation. Annals of Probability 24, 1036-1048.

43. Appel, M. J. and Wierman, J. C. (1987). On the absence of AB percolation in bipartite graphs. Journal of Physics A: Mathematical and General 20 $2527-2531$.

44. Appel, M. J. and Wierman, J. C. (1987). Infinite AB percolation clusters exist on the triangular lattice. Journal of Physics A: Mathematical and General 20, 2533-2537.

45. Appel, M. J. and Wierman, J. C. (1993). AB percolation on bond decorated graphs. Journal of Applied Probability 30, 153-166.

46. Ashkin, J. and Teller, E. (1943). Statistics of two-dimensional lattices with four components. The Physical Review 64, 178-184.

47. Barlow, M. T., Pemantle, R., and Perkins, E. (1997). Diffusion-limited aggregation on a homogenous tree. Probability Theory and Related Field $107,1-60$.

48. Barsky, D. J. and Aizenman, M. (1991). Percolation critical exponents under the triangle condition. Annals of Probability 19, 1520-1536.

49. Barsky, D. J., Grimmett, G. R., and Newman, C. M. (1991). Dynamic renormalization and continuity of the percolation transition in orthants. Spatial Stochastic Processes (K. S. Alexander and J. C. Watkins, eds.), Birkhäuser, Boston, pp. 37-55.

50. Barsky, D. J., Grimmett, G. R., and Newman, C. M. (1991). Percolation in half spaces: equality of critical probabilities and continuity of the percolation probability. Probability Theory and Related Fields 90, 111-148.

51. Benjamini, I. (1996). Percolation on groups (to appear).

52. Benjamini, I. and Kesten, H. (1995). Percolation of arbitrary words in $\{0,1\}^{\mathbb{N}}$. Annals of Probability 23, 1024-1060.

53. Benjamini, I., Pemantle, R., and Peres, Y. (1995). Martin capacity fo Markov chains. Annals of Probability 23, 1332-1346.

54. Benjamini, I., Pemantle, R., and Peres, Y. (1997). Unpredictable paths and percolation (to appear)
55. Benjamini, I. and Peres, Y. (1992). Random walks on a tree and capacity in the interval. Annales de l'Institut Henri Poincaré 28, 557-592.

56. Benjamini, I. and Peres, Y. (1994). Tree-indexed random walks on groups and first passage percolation. Probability Theory and Related Fields $\mathbf{9 8}$ $91-112$

57. Benjamini, I. and Schramm, O. (1996). Conformal invariance of Voronoi percolation (to appear).

58. Berg, J. van den (1985). Disjoint occurrences of events: results and conjectures. Particle Systems, Random Media and Large Deviation (R. T. Durrett, ed.), Contemporary Mathematics no. 41, American Mathematical Society, Providence, R. I., pp. 357-361.

59. Berg, J. van den (1993). A uniqueness condition for Gibbs measures, with application to the 2-dimensional Ising anti-ferromagnet. Communications in Mathematical Physics 152, 161-166.

60. Berg, J. van den (1996). A constructive mixing condition for 2-D Gibbs measures with random interactions (to appear).

61. Berg, J. van den (1996). A note on disjoint-occurrence inequalities fo marked Poisson point processes. Journal of Applied Probability 33, 420-426.

62. Berg, J. van den (1997). Some reflections on disjoint occurrences of events (to appear).

63. Berg, J. van den and Ermakov, A. (1996). A new lower bound for the critica probability of site percolation on the square lattice. Random Structures and Algorithms, 199-212.

64. Berg, J. van den and Fiebig, U. (1987). On a combinatorial conjecture concerning disjoint occurrences of events. Annals of Probability 15, 354-374.

65. Berg, J. van den, Grimmett, G. R., and Schinazi, R. B. (1997). Dependent random graphs and spatial epidemics (to appear).

66. Berg, J. van den and Keane, M. (1985). On the continuity of the percolation probability function. Particle Systems, Random Media and Large Deviations (R. T. Durrett, ed.), Contemporary Mathematics Series, vol. 26, AMS, Providence, R. I., pp. 61-65.

67. Berg, J. van den and Kesten, H. (1985). Inequalities with applications to percolation and reliability. Journal of Applied Probability 22, 556-569.

68. Berg, J. van den and Kesten, H. (1993). Inequalities for the time constant in first-passage percolation. Advances in Applied Probability 3, 56-80.

69. Berg, J. van den and Maes, C. (1994). Disagreement percolation in the study of Markov fields. Annals of Probability 22, 749-763.

70. Berg, J. van den and Meester, R. (1991). Stability properties of a flow process in graphs. Random Structures and Algorithms 2, 335-341.

71. Berg, J. van den and Steif, J. (1994). Percolation and the hard-core lattice gas model. Stochastic Processes and their Applications 49, 179-197.

72. Bezuidenhout, C. E. and Grimmett, G. R. (1990). The critical contact process dies out. Annals of Probability 18, 1462-1482.

73. Bezuidenhout, C. E. and Grimmett, G. R. (1991). Exponential decay fo subcritical contact and percolation processes. Annals of Probability 19, $984-1009$ 
74. Bezuidenhout, C. E. and Grimmett, G. R. (1996). A central limit theorem for random walks in random labyrinths (to appear).

75. Bezuidenhout, C. E., Grimmett, G. R., and Kesten, H. (1993). Strict inequality for critical values of Potts models and random-cluster processes. Communications in Mathematical Physics 158, 1-16.

76. Bezuidenhout, C. E., Grimmett, G. R., and Löffler, A. (1996). Minimal spanning tree analysis of site percolation clusters (to appear).

77. Bollobás, B., Grimmett, G. R., and Janson, S. (1996). The random-cluster process on the complete graph. Probability Theory and Related Fields 104 283-317.

78. Borgs, C. and Chayes, J. T. (1996). The covariance matrix of the Potts model: a random cluster analysis. Journal of Statistical Physics $\mathbf{8 2}$ $1235-1297$

79. Borgs, C., Chayes, J. T., Kesten, H., and Spencer, J. (1996). The birth of the infinite cluster (to appear)

80. Bramson, M., Durrett, R. T., and Schonmann, R. H. (1991). The contact process in a random environment. Annals of Probability 19, 960-983.

81. Broadbent, S. R. and Hammersley, J. M. (1957). Percolation processes Crystals and mazes. Proceedings of the Cambridge Philosophical Society $\mathbf{5 3}$ 629-641.

82. Bunimovitch, L. A. and Troubetzkoy, S. E. (1992). Recurrence properties of Lorentz lattice gas cellular automata. Journal of Statistical Physics $\mathbf{6 7}$, $289-302$

83. Burton, R. M. and Keane, M. (1989). Density and uniqueness in percolation. Communications in Mathematical Physics 121, 501-505.

84. Burton, R. M. and Meester, R. (1993). Long range percolation in stationary point processes. Random Structures and Algorithms 4, 177-190.

85. Burton, R. M. and Pemantle, R. (1993). Local characteristics, entropy and limit theorems for uniform spanning trees and domino tilings via transferimpedances. Annals of Probability 21, 1329-1371.

86. Campanino, M., Chayes, J. T., and Chayes, L. (1991). Gaussian fluctuations of connectivities in the subcritical regime of percolation. Probability Theory and Related Fields 88, 269-341.

87. Cardy, J. (1992). Critical percolation in finite geometries. Journal of Physics A: Mathematical and General 25, L201.

88. Chayes, J. T. and Chayes, L. (1987). The mean field bound for the order parameter of Bernoulli percolation,. Percolation Theory and Ergodic Theory of Infinite Particle Systems (H. Kesten, ed.), Springer-Verlag, New York, pp. $49-71$.

89. Chayes, J. T. and Chayes, L. (1987). On the upper critical dimension of Bernoulli percolation,. Communications in Mathematical Physics 113, 27-48.

90. Chayes, J. T. and Chayes, L. (1989). The large- $N$ limit of the threshold values in Mandelbrot's fractal percolation process. Journal of Physics A Mathematical and General 22, L501-L506.

91. Chayes, J. T., Chayes, L., and Durrett, R. T. (1986). Critical behavior of the two-dimensional first passage time. Journal of Statistical Physics $\mathbf{4 5}$ $933-948$.
92. Chayes, J. T., Chayes, L., and Durrett, R. T. (1988). Connectivity properties of Mandelbrot's percolation process. Probability Theory and Related Fields $\mathbf{7 7}, 307-324$.

93. Chayes, J. T., Chayes, L., Fisher, D. S., and Spencer, T. (1989). Correlation length bounds for disordered Ising ferromagnets. Communications in Mathematical Physics 120, 501-523.

94. Chayes, J. T., Chayes, L., Grannan, E., and Swindle, G. (1991). Phase transitions in Mandelbrot's percolation process in 3 dimensions. Probability Theory and Related Fields 90, 291-300.

95. Chayes, J. T., Chayes, L., Grimmett, G. R., Kesten, H., and Schonmann, R. H. (1989). The correlation length for the high density phase of Bernoulli percolation. Annals of Probability 17, 1277-1302.

96. Chayes, J. T., Chayes, L., and Kotecky, R. (1995). The analysis of the Widom-Rowlinson model by stochastic geometric methods. Communication in Mathematical Physics 172, 551-569.

97. Chayes, J. T., Chayes, L., and Newman, C M. (1987). Bernoulli percolation above threshold: an invasion percolation analysis. Annals of Probability 15, $1272-1287$

98. Chayes, J. T., Chayes, L., and Schonmann, R. H. (1987). Exponential decay of connectivities in the two dimensional Ising model. Journal of Statistical Physics 49, 433-445.

99. Chayes, L. (1991). On the critical behavior of the first passage time in $d=3$. Helvetica Physica Acta 64, 1055-1069.

100. Chayes, L. (1993). The density of Peierls contours in $d=2$ and the height of the wedding cake. Journal of Physics A: Mathematical and General $\mathbf{2 6}$, L481-L488.

101. Chayes, L. (1995). On the absence of directed fractal percolation. Journal of Physics A: Mathematical and General 28, L295-L301.

102. Chayes, L. (1995). Aspects of the fractal percolation process. Fractal Geometry and Stochastics (C. Bandt, S. Graf and M. Zähle, eds.), Birkhäuser, Boston, pp. 113-143.

103. Chayes, L. (1996). On the length of the shortest crossing in the super-critical phase of Mandelbrot's percolation process. Stochastic Processes and their Applications 61, 25-43.

104. Chayes, L. (1996). Percolation and ferromagnetism on $\mathbb{Z}^{2}$ : the $q$-state Potts cases (to appear).

105. Chayes, L., Kotecky, R., and Shlosman, S. B. (1995). Aggregation and intermediate phases in dilute spin-systems. Communications in Mathematical Physics 171, 203-232.

106. Chayes, L. and Winfield, C. (1993). The density of interfaces: a new first passage problem. Journal of Applied Probability 30, 851-862.

107. Chow, Y. S. and Teicher, H. (1978). Probability Theory. Springer, Berlin.

108. Clifford, P. (1990). Markov random fields in statistics. Disorder in Physical Systems (G. R. Grimmett and D. J. A. Welsh, eds.), Oxford University Press, Oxford, pp. 19-32.

109. Cohen, E. G. D. (1991). New types of diffusions in lattice gas cellular automata. Microscopic Simulations of Complex Hydrodynamical Phenomena 
(M. Mareschal and B. L. Holian, eds.), Plenum Press, New York, pp. $137-152$.

110. Cohen. E. G. D and Wang, F. (1995). New results for diffusion in Lorent lattice gas cellular automata. Journal of Statistical Physics 81, 445-466.

111. Cohen, E. G. D. and Wang, F. (1995). Novel phenomena in Lorentz lattice gases. Physica A 219, 56-87.

112. Cox, J. T., Gandolfi, A., Griffin, P., and Kesten, H. (1993). Greedy lattice animals I: Upper bounds. Advances in Applied Probability 3, 1151-1169.

113. Dekking, F. M. and Grimmett, G. R. (1988). Superbranching processes and projections of random Cantor sets. Probability Theory and Related Fields $\mathbf{7 8}$ 335-355.

114. Dekking, F. M. and Meester, R. W. J. (1990). On the structure of Mandelbrot's percolation process and other random Cantor sets. Journal of Statistical Physics 58, 1109-1126.

115. DeMasi, A., Ferrari, P. A., Goldstein, S., and Wick, W. D. (1985). Invariance principle for reversible Markov processes with application to diffusion in the percolation regime. Particle Systems, Random Media and Large Deviations (R. T. Durrett, ed.), Contemporary Mathematics no. 41, American Mathematical Society, Providence, R. I., pp. 71-85.

116. DeMasi, A., Ferrari, P. A., Goldstein, S., and Wick, W. D. (1989). An invariance principle for reversible Markov processes. Applications to random motion

. Deuschel, J.-D. and Pisztora, A. (1996). Surface order large deviations for high-density percolation. Probability Theory and Related Fields 104, 467-482.

118. Doyle, P. G. and Snell, E. L. (1984). Random Walks and Electric Networks. Carus Mathematical Monograph no. 22. AMA, Washington, D. C.

119. Durrett, R. T. (1988). Lecture Notes on Particle Systems and Percolation. Wadsworth and Brooks/Cole, Pacific Grove, California.

120. Durrett, R. T. (1991). The contact process, 1974-1989. Mathematics of Random Media Blacksburg, VA, Lectures in Applied Mathematics, vol. 27, AMS, pp. $1-18$

121. Durrett, R. T. (1995). Ten lectures on particle systems. Ecole d'Eté de Probabilités de Saint Flour XXIII-1993 (P. Bernard, ed.), Lecture Notes in Mathematics no. 1608, Springer, Berlin, pp. 97-201.

122. Durrett, R. T. and Kesten, H. (1990). The critical parameter fo connectedness of some random graphs. A Tribute to Paul Erdös (A. Baker, B. Bollobás, and A. Hajnal, eds.), Cambridge University Press, Cambridge, pp. $161-176$

123. Durrett, R. T. and Neuhauser, C. (1991). Epidemics with recovery. Advances in Applied Probability 1, 189-206.

124. Durrett, R. T. and Schonmann, R. H. (1988). The contact process on a finite set II. Annals of Probability 16, 1570-1583.

25. Durrett, R. T. and Schonmann, R. H. (1988). Large deviations for the contact process and two dimensional percolation. Probability Theory and Related Fields $\mathbf{7 7}, 583-603$
126. Durrett, R. T., Schonmann, R. H., and Tanaka, N. I. (1989). The contact process on a finite set III. The critical case. Annals of Probability 17, $1303-1321$.

127. Durrett, R. T., Schonmann, R. H., and Tanaka, N. I. (1989). Correlation lengths for oriented percolation. Journal of Statistical Physics 55, 965-979

128. Edwards, R. G. and Sokal, A. D. (1988). Generalization of the FortuinKasteleyn-Swendsen-Wang representation and Monte Carlo algorithm. The Physical Review D 38, 2009-2012.

129. Ehrenfest, P. (1959). Collected Scientific Papers (M. J. Klein, ed.). NorthHolland, Amsterdam.

130. Enter, A. C. D. van and Hollander, F. den (1993). Interacting particle systems and Gibbs measures, Lecture notes for Master Class 1992-1993, Mathematical Research Institute, The Netherlands.

131. Falconer, K. J. (1986). Random fractals. Mathematical Proceedings of the Cambridge Philosophical Society 100, 559-582.

132. Falconer, K. J. (1986). Sets with prescribed projections and Nikodym sets. Proceedings of the London Mathematical Society 53, 48-64.

133. Falconer, K. J. (1989). Projections of random Cantor sets. Journal of Theoretical Probability 2, 65-70.

134. Falconer, K. J. and Grimmett, G. R. (1992). On the geometry of random Cantor sets and fractal percolation. Journal of Theoretical Probability $\mathbf{5}$, 465-485; Erratum (1994) 7, 209-210.

135. Fernández, R., Fröhlich, J., and Sokal, A. D. (1992). Random Walks, Critical Phenomena, and Triviality in Quantum Field Theory. Springer-Verlag,

136. Fill, J. and Pemantle, R. (1992). Oriented percolation, first-passage percolation and covering times for Richardson's model on the $n$-cube. Annal of Applied Probability 3, 593-629.

137. Fiocco, M. and Zwet, W. R. van (1996). Consistent estimation for the supercritical contact process (to appear).

138. Fisher, M. E. (1961). Critical probabilities for cluster size and percolation problems. Journal of Mathematical Physics 2, 620-627.

139. Fontes, L. and Newman, C. M. (1993). First passage percolation for random colorings of $\mathbb{Z}^{d}$. Annals of Applied Probability 3, 746-762; Erratum 4, 254

140. Fortuin, C. M. (1971). On the random-cluster model. Doctoral thesis, University of Leiden.

141. Fortuin, C. M. (1972). On the random cluster model. II. The percolation model. Physica 58, 393-418.

142. Fortuin, C. M. (1972). On the random cluster model. III. The simple random-cluster process. Physica 59, 545-570.

143. Fortuin, C. M. and Kasteleyn, P. W. (1972). On the random cluster model. I. Introduction and relation to other models. Physica 57, 536-564.

144. Fortuin, C. M., Kasteleyn, P. W., and Ginibre, J. (1971). Correlation inequalities on some partially ordered sets. Communications in Mathematica Physics 22, 89-103. 
145. Friedgut, E. and Kalai, G. (1996). Every monotone graph property has a sharp threshold. Proceedings of the American Mathematical Society 124 2993-3002.

146. Gandolfi, A., Grimmett, G. R., and Russo, L. (1988). On the uniqueness of the infinite open cluster in the percolation model. Communications in Mathematical Physics 114, 549-552.

147. Gandolfi, A., Keane, M., and Newman, C. M. (1992). Uniqueness of the infinite component in a random graph with applications to percolation and spin glasses. Probability Theory and Related Fields 92, 511-527.

148. Gandolfi, A. and Kesten, H. (1994). Greedy lattice animals II: Linear growth. Advances in Applied Probability 4, 76-107.

149. Gandolfi, A., Newman, C. M., and Stein, D. L. (1993). Exotic states in long range spin glasses. Communications in Mathematical Physics 157, 371-387.

150. Georgii, H.-O. (1988). Gibbs Measures and Phase Transitions. Walter de Gruyter, Berlin.

51. Gordon, D. M. (1991). Percolation in high dimensions. Bulletin of the London Mathematical Society 44, 373-384.

152. Graf, S. (1987). Statistically self-similar fractals. Probability Theory and Related Fields 74, 357-392.

153. Graf, S., Mauldin, R. D., and Williams, S. C. (1987). Exact Hausdorff dimension in random recursive constructions. Memoirs of the American Mathematical Society $\mathbf{3 8 1}$.

154. Griffeath, D. (1979). Additive and Cancellative Interacting Particle Systems. Lecture Notes in Mathematics, vol. 724. Springer, Berlin.

155. Grimmett, G. R. (1978). The rank functions of large random lattices. Journal of London Mathematical Society 18, 567-575.

156. Grimmett, G. R. (1989). Percolation. Springer-Verlag, Berlin.

157. Grimmett, G. R. (1993). Differential inequalities for Potts and randomcluster processes. Cellular Automata and Cooperative Systems (N. Boccara et al., eds.), Kluwer, Dordrecht, pp. 227-236.

158. Grimmett, G. R. (1994). Potts models and random-cluster processes with many-body interactions. Journal of Statistical Physics 75, 67-121.

159. Grimmett, G. R. (1994). The random-cluster model. Probability, Statistics and Optimisation (F. P. Kelly, ed.), John Wiley \& Sons, Chichester, pp. $49-63$.

160. Grimmett, G. R. (1994). Percolative problems. Probability and Phase Transition (G. R. Grimmett, ed.), Kluwer, Dordrecht, pp. 69-86.

161. Grimmett, G. R. (1994). Probability and Phase Transition. editor. Kluwer, Dordrecht.

162. Grimmett, G. R. (1995). Comparison and disjoint-occurrence inequalities for random-cluster models. Journal of Statistical Physics 78, 1311-1324.

163. Grimmett, G. R. (1995). The stochastic random-cluster process and the uniqueness of random-cluster measures. Annals of Probability 23, 1461-1510.

164. Grimmett, G. R., Kesten, H., and Zhang, Y. (1993). Random walk on the infinite cluster of the percolation model. Probability Theory and Related Fields 96, 33-44.
165. Grimmett, G. R. and Marstrand, J. M. (1990). The supercritical phase of percolation is well behaved. Proceedings of the Royal Society (London), Series A 430, 439-457.

166. Grimmett, G. R., Menshikov, M. V., and Volkov, S. E. (1996). Random walks in random labyrinths. Markov Processes and Related Fields 2, 69-86.

167. Grimmett, G. R. and Newman, C. M. (1990). Percolation in $\infty+$ 1 dimensions. Disorder in Physical Systems (G. R. Grimmett an D. J. A. Welsh, eds.), Clarendon Press, Oxford, pp. 167-190.

168. Grimmett, G. R. and Piza, M. S. T. (1996). Decay of correlations in subcritical Potts and random-cluster models (to appear).

169. Grimmett, G. R. and Stacey, A. M. (1996). Inequalities for critical probabilities of site and bond percolation (to appear).

170. Grimmett, G. R. and Stirzaker, D. R. (1992). Probability and Random Processes. Oxford University Press, Oxford.

171. Häggström, O. (1996). The random-cluster model on a homogeneous tree. Probability Theory and Related Fields 104, 231-253.

172. Häggström, O. and Pemantle, R. (1995). First-passage percolation and model for competing spatial growth (to appear).

173. Häggström, O., Peres, Y., and Steif, J. (1995). Dynamical percolation (to appear).

174. Hammersley, J. M. (1957). Percolation processes. Lower bounds for the critical probability. Annals of Mathematical Statistics 28, 790-795.

175. Hammersley, J. M. (1959). Bornes supérieures de la probabilité critique dans un processus de filtration. Le Calcul de Probabilités et ses Applications, CNRS, Paris, pp. 17-37.

176. Hara, T. and Slade, G. (1989). The mean-field critical behaviour of percolation in high dimensions. Proceedings of the IXth International Congress on Mathematical Physics (B. Simon, A. Truman, I. M. Davies, eds.), Adam Hilger, Bristol, pp. 450-453.

177. Hara, T. and Slade, G. (1989). The triangle condition for percolation. Bulletin of the American Mathematical Society 21, 269-273.

178. Hara, T. and Slade, G. (1990). Mean-field critical behaviour for percolation in high dimensions. Communications in Mathematical Physics 128, 333-391.

179. Hara, T. and Slade, G. (1994). Mean-field behaviour and the lace expansion. Probability and Phase Transition (G. R. Grimmett, ed.), Kluwer, Dordrecht, pp. $87-122$.

180. Hara, T. and Slade, G. (1995). The self-avoiding walk and percolation critical points in high dimensions. Combinatorics, Probability, Computing 4, 197-215.

181. Harris, T. E. (1960). A lower bound for the critical probability in a certain percolation process. Proceedings of the Cambridge Philosophical Society $\mathbf{5 6}$ $13-20$

182. Hauge, E. H. and Cohen, E. G. D. (1967). Normal and abnormal effects in Ehrenfest wind-tree model. Physics Letters 25A, 78-79.

183. Hawkes, J. (1981). Trees generated by a simple branching process. Journal of the London Mathematical Society 24, 373-384. 
184. Higuchi, Y. (1989). A remark on the percolation for the 2D Ising model. Osaka Journal of Mathematics 26, 207-224.

185. Higuchi, Y. (1991). Level set representation for the Gibbs state of the ferromagnetic Ising model. Probability Theory and Related Fields 90 203-221.

186. Higuchi, Y. (1992). Percolation (in Japanese). Yuseisha/Seiunsha.

187. Higuchi, Y. (1993). A sharp transition for the two-dimensional Ising percolation. Probability Theory and Related Fields 97, 489-514.

188. Higuchi, Y. (1993). Coexistence of infinite *-clusters II: Ising percolation in two dimensions. Probability Theory and Related Fields 97, 1-34.

189. Higuchi, Y. (1996). Ising model percolation. Sugaku Expositions, vol. 47, pp. $111-124$.

190. Higuchi, Y. (1996). Mixing condition and percolation for Ising models (to appear).

191. Hintermann, D., Kunz, H., and Wu, F. Y. (1978). Exact results for the Pott model in two dimensions. Journal of Statistical Physics 19, 623-632.

192. Holley, R. (1974). Remarks on the FKG inequalities. Communications in Mathematical Physics 36, 227-231.

193. Imbrie, J. and Newman, C. M. (1988). An intermediate phase with slow decay of correlations in one dimensional $1 /|x-y|^{2}$ percolation, Ising and Potts models. Communications in Mathematical Physics 118, 303-336.

194. Ising, E. (1925). Beitrag zur Theorie des Ferromagnetismus. Zeitschrift für Physik 31, 253-258.

195. Jacobs, D. J. and Thorpe, M. F. (1995). Generic rigidity percolation: the pebble game. Physical Review Letters 75, 4051-4054.

196. Jacobs, D. J. and Thorpe, M. F. (1996). Rigidity percolation in two dimensions. The pebble game. Physical Review E 53, 3682-3693.

197. Jagers, P. (1975). Branching Processes with Biological Applications. John Wiley, Chichester.

198. Kantor, T, and Hassold, G. N. (1988). Topological entanglements in the percolation problem. Physical Review Letters 60, 1457-1460.

199. Kasteleyn, P. W. and Fortuin, C. M. (1969). Phase transitions in lattice systems with random local properties. Journal of the Physical Society of Japan 26, 11-14, Supplement.

200. Kesten, H. (1980). The critical probability of bond percolation on the squar lattice equals $\frac{1}{2}$. Communications in Mathematical Physics 74, 41-59.

201. Kesten, H. (1980). On the time constant and path length of first-passage percolation. Advances in Applied Probability 12, 848-863.

202. Kesten, H. (1982). Percolation Theory for Mathematicians. Birkhäuser, Boston.

203. Kesten, H. (1986). Aspects of first-passage percolation. Ecole d'Eté de Probabilités de Saint Flour XIV-1984 (P. L. Hennequin, ed.), Lecture Notes in Mathematics no. 1180, Springer, Berlin, pp. 125-264.

204. Kesten, H. (1987). Scaling relations for 2D-percolation. Communications in Mathematical Physics 109, 109-156.
205. Kesten, H. (1987). A scaling relation at criticality for 2D-percolation. Percolation Theory and Ergodic Theory of Infinite Particle Systems (H. Kesten, ed.), Springer, Berlin, pp. 203-212.

206. Kesten, H. (1987). Percolation theory and first-passage percolation. Annals of Probability 15, 1231-1271.

207. Kesten, H. (1988). Recent results in rigorous percolation theory. Astérisque $-158$

208. Kesten, H. (1988). Correlation length and critical probabilities of slabs for percolation, preprint.

209. Kesten, H. (1990). Asymptotics in high dimensions for percolation. Disorder in Physical Systems (G. R. Grimmett and D. J. A. Welsh, eds.), Clarendon Press, Oxford.

210. Kesten, H. (1991). Asymptotics in high dimensions for the Fortuin-Kasteleyn random cluster mode. Spatial Stochastic Processes (K. S. Alexander and J. C. Watkins, eds.), Birkhäuser, Boston, pp. 57-85.

211. Kesten, H. (1992). Connectivity of certain graphs on halfspaces, quarte spaces, .... Probability Theory (L. H. Y. Chen, K. P. Choi, K. Hu, and J.-H. Lou, eds.), Walter de Gruyter, Berlin, pp. 91-104.

212. Kesten, H. (1992). First- and last-passage percolation. Proceedings of Statistics Conference in Rio de Janeiro, Brasil.

213. Kesten, H. and Schonmann, R. H. (1990). Behavior in large dimensions of the Potts and Hisenberg models. Revieus in Mothematical Physics 1 , $147-182$

214. Kesten, H. and Zhang, Y. (1987). Strict inequalities for some critical exponents in two-dimensional percolation. Journal of Statistical Physics 46, 1031-1055.

215. Kesten, H. and Zhang, Y. (1990). The probability of a large finite cluster in supercritical Bernoulli percolation. Annals of Probability 18, 537-555.

216. Kesten, H. and Zhang, Y. (1993). The tortuosity of occupied crossings of a box in critical percolation. Journal of Statistical Physics 70, 599-611.

217. Kesten, H. and Zhang, Y. (1997). A central limit theorem for 'critical' first passage percolation in two dimensions. Probability Theory and Related Field $07,137-160$.

218. Kihara, T., Midzuno, Y., and Shizume, J. (1954). Statistics of twodimensional lattices with many components. Journal of the Physical Society of Japan $\mathbf{9}, 681-687$.

219. Kipnis, C. and Varadhan, S. R. S. (1986). Central limit theorem for additive functionals of reversible Markov processes and applications to simple exclusion. Communications in Mathematical Physics 104, 1-19.

220. Klein, A. (1994). Multiscale analysis in disordered systems: Percolation and contact process in a random environment. Probability and Phase Transition G. R. Grimmett, ed.), Kluwer, Dordrecht, pp. 139-152.

221. Klein, A. (1994). Extinction of contact and percolation processes in a random environment. Annals of Probability 22, 1227-1251.

222. Kotecký, R. (1994). Geometric representation of lattice models and large volume asymptotics. Probability and Phase Transition (G. R. Grimmett, ed.), Kluwer, Dordrecht, pp. 153-176. 
223. Kotecký, R. and Shlosman, S. (1982). First order phase transitions in large entropy lattice systems. Communications in Mathematical Physics $\mathbf{8 3}$ $493-515$.

224. Kuulasmaa, K. (1982). The spatial general epidemic and locally dependent random graphs. Journal of A pplied Probability 19,745-758.

225. Laanait, L., Messager, A., Miracle-Sole, S., Ruiz, J., and Shlosman, S. (1991). Interfaces in the Potts model I: Pirogov-Sinai theory of the FortuinKasteleyn representation. Communications in Mathematical Physics 140, 81-91.

226. Laanait, L., Messager, A., and Ruiz, J. (1986). Phase coexistence and surface tensions for the Potts model. Communications in Mathematical Physics 105, $527-545$.

227. Langlands, R. P. (1993). Dualität bei endlichen Modellen der Perkolation. Mathematische Nachrichten 160, 7-58.

228. Langlands, R. P. (1996). An essay on the dynamics and statistics of critical field theories. 50th anniversary volume of the Canadian Mathematical Society (to appear).

229. Langlands, R. P. and Lafortune, M.-A. (1994). Finite models for percolation. Contemporary Mathematics 177, 227-246.

230. Langlands, R., Pichet, C., Pouliot, P., and Saint-Aubin, Y. (1992). On the universality of crossing probabilities in two-dimensional percolation. Journal of Statistical Physics 67, 553-574.

231. Langlands, R., Pouliot, P., and Saint-Aubin, Y. (1994). Conformal invariance in two-dimensional percolation. Bulletin of the American Mathematical Society 30, 1-61.

232. Lebowitz, J. and Martin-Löf, A. (1972). On the uniqueness of the equilibrium state for Ising spin systems. Communications in Mathematical Physics 25, 276-282.

233. Licea, C. and Newman, C. M. (1996). Geodesics in two-dimensional firstpassage percolation. Annals of Probability 24, 399-410.

234. Licea, C., Newman, C. M., and Piza, M. S. T. (1995). Superdiffusivity in first-passage percolation (to appear).

235. Lieb, E. H. (1980). A refinement of Simon's correlation inequality. Communications in Mathematical Physics 77, 127-135.

236. Liggett, T. M. (1985). Interacting Particle Systems. Springer-Verlag, Berlin.

237. Liggett, T. M. (1991). Spatially inhomogeneous contact processes. Spatial Stochastic Processes (K. S. Alexander and J. C. Watkins, eds.), Birkhäuser, Boston, pp. 105-140.

238. Liggett, T. M. (1991). The periodic threshold contact process. Random Walks, Brownian Motion and Interacting Particle Systems (R. T. Durrett and H. Kesten, eds.), Birkhäuser, Boston, pp. 339-358.

239. Liggett, T. M. (1992). The survival of one-dimensional contact processes in random environments. Annals of Probability 20, 696-723.

240. Liggett, T. M. (1994). Coexistence in threshold voter models. Annals of Probability 22, 764-802.

241. Liggett, T. M. (1995). Improved upper bounds for the contact process critical value. Annals of Probability 23, 697-723.
242. Liggett, T. M. (1995). Survival of discrete time growth models, with applications to oriented percolation. Annals of Applied Probability $\mathbf{5}$, $613-636$

243. Liggett, T. M. (1996). Multiple transition points for the contact process on the binary tree. Annals of Probability 24, 1675-1710.

244. Liggett, T. M., Schonmann, R. H., and Stacey, A. (1997). Domination by product measures. Annals of Probability 25 (to appear).

245. Lorentz, H. A. (1905). The motion of electrons in metallic bodies, I, II, and III. Koninklijke Akademie van Wetenschappen te Amsterdam, Section of Sciences 7, 438-453, 585-593, 684-691.

246. Luczak, T. and Wierman, J. C. (1988). Critical probability bounds for twodimensional site percolation models. Journal of Physics A: Mathematical and General 21, 3131-3138.

247. Luczak, T. and Wierman, J. C. (1989). Counterexamples in AB percolation. Journal of Physics A: Mathematical and General 22, 185-191.

248. Lyons, R. (1990). Random walks and percolation on trees. Annals of Probability 18, 931-958.

249. Lyons, R. (1992). Random walks, capacity and percolation on trees. Annals of Probability 20, 2043-2088.

250. Lyons, R. and Pemantle, R. (1992). Random walk in a random environment and first-passage percolation on trees. Annals of Probability 20, 125-137.

251. Lyons, T. (1983). A simple criterion for transience of a reversible Markov chain. Annals of Probability 11, 393-402.

252. Madras, N., Schinazi, R. B., and Schonmann, R. H. (1994). On the critical behavior of the contact process in deterministic inhomogeneous environment. Annals of Probability 22, 1140-1159.

253. Madras, N. and Slade, G. (1993). The Self-Avoiding Walk. Birkhäuser, Boston.

254. Mandelbrot, B. (1983). The Fractal Geometry of Nature. W. H. Freeman, San Francisco.

255. Martirosian, D. H. (1986). Translation invariant Gibbs states in the $q$-state Potts model. Communications in Mathematical Physics 105, 281-290.

256. Mauldin, R. D. and Williams, S. C. (1986). Random recursive constructions: asymptotic geometry and topological properties. Transactions of the American Mathematical Society 295, 325-346.

257. Meester, R. (1989). An algorithm for calculating critical probabilities and percolation functions in percolation models defined by rotations. Ergodic Theory and Dynamical Systems 8, 495-509.

258. Meester, R. (1992). Connectivity in fractal percolation. Journal of Theoretical Probability 5, 775-789.

259. Meester, R. (1994). Uniqueness in percolation theory; a review. Statistica Neerlandica 48, 237-252.

260. Meester, R. (1995). Equality of critical densities in continuum percolation. Journal of Applied Probability 32, 90-104.

261. Meester, R. and Häggström, O. (1995). Asymptotic shapes in stationary first passage percolation. Annals of Probability 23, 1511-1522. 
262. Meester, R. and Häggström, O. (1995). Nearest neighbour and hard sphere models in continuum percolation (to appear)

263. Meester, R. and Nowicki, T. (1989). Infinite clusters and critical values in two-dimensional circle percolation. Israel Journal of Mathematics 68, 63-81.

264. Meester, R., Penrose, M., and Sarkar, A. (1995). The random connection model in high dimensions (to appear)

265. Meester, R., Roy, R., and Sarkar, A. (1994). Non-universality and continuity of the critical covered volume fraction in continuum percolation. Journal of Statistical Physics 75, 123-134.

266. Meester, R. and Steif, J. (1996). On the continuity of the critical value for long range percolation in the exponential case. Communications in Mathematical Physics 180, 483-504.

267. Meester, R. and Steif, J. (1995). Consistent estimation of percolation quantities (to appear)

268. Menshikov, M. V. (1986). Coincidence of critical points in percolation problems. Soviet Mathematics Doklady 33, 856-859

269. Menshikov, M. V. (1987). Quantitative estimates and rigorous inequalities for critical points of a graph and its subgraphs. Theory of Probability and its Applications 32, 544-547.

270. Menshikov, M. V. (1987). Strict inequalities for critical probabilities of a regular graph and its subgraphs. Uspekhy Math. Nauk 42, 239-240.

271. Menshikov, M. V., Molchanov, S. A., and Sidorenko, A. F. (1986). Percolation theory and some applications. Itogi Nauki i Techniki (Series of Probability Theory, Mathematical Statistics, Theoretical Cybernetics) 24, $53-110$

272. Menshikov, M. V. and Pelikh, K. D. (1989). Percolation with several types of defects. Estimates of the critical probability on the square lattice, 778-785 (in translation). Mathematical Notes 46, 38-47 (in Russian).

273. Menshikov, M. V. and Pelikh, K. D. (1993). Percolation method in a system of numerous particles. Proceeding of Third Conference on Probability Method in Discrete Mathematics, Petrozavodsk, TVP/VSP, pp. 329-336.

274. Menshikov, M. V. and Zuev, S. A. (1993). Percolation method in a system of numerous particles. Probabilistic Methods in Discrete Mathematics V. F. Kolchin, ed.), TVP/VSP, pp. 329-336

275. Menshikov, M. V. and Zuev, S. A. (1993). Models of $\rho$-percolation. Probabilistic Methods in Discrete Mathematics (V. F. Kolchin, ed.), TVP Science Publishers, pp. 337-347.

276. Morrow, G., Schinazi, R., and Zhang, Y. (1994). The critical contact process on a homogeneous tree. Journal of Applied Probability 31, 250-255.

277. Nash-Williams, C. St. J. A. (1959). Random walks and electric currents in networks. Proceedings of the Cambridge Philosophical Society 55, 181-194.

278. Newman, C. M. (1986). Some critical exponent inequalities for percolation. Journal of Statistical Physics 45, 359-368.

279. Newman, C. M. (1986). Percolation theory: a selective survey of rigorous results. Advances in Multiphase Flow and Related Problems G. Papanicolaou, ed.), SIAM, Philadelphia, pp. 147-167.
280. Newman, C. M. (1987). Inequalities for $\gamma$ and related critical exponents in short and long range percolation. Percolation Theory and Ergodic Theory of Infinite Particle Systems (H. Kesten, ed.), Springer, Berlin, pp. 229-244.

281. Newman, C. M. (1987). Another critical exponent inequality for percolation: $\beta \geq 2 / \delta$. Journal of Statistical Physics 47, 695-699.

282. Newman, C. M. (1989). The $1 / r^{2}$ phase transition: a review. Proceedings of the IXth International Congress on Mathematical Physics (B. Simon, A. Truman, and I. M. Davies, eds.), Adam Hilger, Bristol, pp. 377-379.

283. Newman, C. M. (1991). Ising models and dependent percolation. Topics in Statistical Dependence (H. W. Block, A. R. Sampson, and T. H. Savits, eds.) IMS Lecture Notes, Monograph Series, vol. 16, pp. 395-401.

284. Newman, C. M. (1991). Topics in percolation. Mathematics of Random Media (W. Kohler and B. White, eds.), vol. 27, American Mathematical Society, Providence, pp. 41-51.

285. Newman, C. M. (1994). Disordered Ising systems and random cluster representations. Probability and Phase Transition (G. R. Grimmett, ed.) Kluwer, Dordrecht, pp. 247-260.

286. Newman, C. M. (1995). A surface view of first-passage percolation. Proceedings of The International Congress of Mathematicians (S. D. Chatterji, ed.), Birkhäuser, Boston, pp. 1017-1023.

287. Newman, C. M. and Piza, M. S. T. (1995). Divergence of shape fluctuations in two dimensions. Annals of Probability 23, 977-1005.

288. Newman, C. M. and Schulman, L. S. (1981). Infinite clusters in percolation models. Journal of Statistical Physics 26, 613-628.

289. Newman, C. M. and Stein, D. L. (1990). Broken symmetry and domain structure in Ising-like systems. Physical Review Letters 65, 460-463.

290. Newman, C. M. and Stein, D. L. (1992). Multiple states and thermodynamic limits in short-ranged Ising spin glass models. Physical Review B $\mathbf{4 6}$, 973-982.

291. Newman, C. M. and Stein, D. L. (1993). Chaotic size dependence in spin glasses. Cellular Automata and Cooperative Systems (N. Boccara, E. Goles, S. Martinez, and P. Picco, eds.). Kluwer, Dordrecht, pp. 525-529.

292. Newman, C. M. and Stein, D. L. (1994). Spin glass model with dimensiondependent ground state multiplicity. Physical Review Letters 72, 2286-2289.

293. Newman, C. M. and Stein, D. L. (1995). Random walk in a strongly nhomogeneous environment and invasion percolation. Annales de l'Institut Henri Poincaré 31, 249-261.

294. Newman, C. M. and Stein, D. L. (1995). Broken ergodicity and the geometry of rugged landscapes. Physical Review E 51, 5228-5238.

295. Newman, C. M. and Stein, D. L. (1996). Non-mean-field behavior of realistic spin glasses. Physical Review Letters 76, 515-518.

296. Newman, C. M. and Stein, D. L. (1996). Ground state structure in a highly disordered spin glass model. Journal of Statistical Physics 82, 1113-1132.

297. Newman, C. M. and Stein, D. L. (1995). Spatial inhomogeneity and thermodynamic chaos (to appear). 
298. Newman, C. M. and Volchan, S. B. (1996). Persistent survival of onedimensional contact processes in random environments. Annals of Probability 24, 411-421.

299. Newman, C. M. and Wu, C. C. (1990). Markov fields on branching planes. Probability Theory and Related Fields 85, 539-552.

300. Onsager, L. (1944). Crystal statistics, I. A two-dimensional model with an order-disorder transition. The Physical Review 65, 117-149.

301. Ornstein, L. S. and Zernike, F. (1915). Accidental deviations of density and opalescence at the critical point of a single substance. Koninklijke Akademie van Wetenschappen te Amsterdam, Section of Sciences 17, 793-806.

302. Orzechowski, M. E. (1996). On the phase transition to sheet percolation in random Cantor sets. Journal of Statistical Physics 82, 1081-1098.

303. Pemantle, R. (1991). Choosing a spanning tree for the integer lattice uniformly. Annals of Probability 19, 1559-1574.

304. Pemantle, R. (1992). The contact process on trees. Annals of Probability 20 2089-2116.

305. Pemantle, R. (1995). Uniform random spanning trees. Topics in Contemporary Probability and its Applications (J. L. Snell, ed.), CRC Press, Boca Raton.

306. Pemantle, R. and Peres, Y. (1994). Planar first-passage percolation times are not tight. Probability and Phase Transition (G. R. Grimmett, ed.), Kluwer, Dordrecht, pp. 261-264.

307. Pemantle, R. and Peres, Y. (1994). Domination between trees and application to an explosion problem. Annals of Probability 22, 180-194.

308. Pemantle, R. and Peres, Y. (1996). On which graphs are all random walks i random environments transient?. Random Discrete Structures (D. Aldous and R. Pemantle, eds.), IMA Vol. Math. Appl. 76, Springer, New York, pp. 207-211.

309. Pemantle, R. and Peres, Y. (1996). Galton-Watson trees with the same mean have the same polar sets. Annals of Probability 23, 1102-1124.

310. Penrose, M. (1996). Continuum percolation and Euclidean minimal spanning trees in high dimensions. Annals of Applied Probability 6, 528-544.

311. Penrose, M. and Pisztora, A. (1996). Large deviations for discrete and continuous percolation. Advances in Applied Probability 28, 29-52.

312. Peres, Y. (1996). Intersection-equivalence of Brownian paths and certain branching processes. Communications in Mathematical Physics 177, 417-434.

313. Peyrière, J. (1978). Mandelbrot random beadsets and birthprocesses with interaction, I.B.M. Research Report RC-7417.

314. Pirogov, S. A. and Sinai, Ya. G. (1975). Phase diagrams of classical lattice systems. Theoretical and Mathematical Physics 25, 1185-1192.

315. Pirogov, S. A. and Sinai, Ya. G. (1976). Phase diagrams of classical lattice systems, continuation. Theoretical and Mathematical Physics 26, 39-49.

316. Pisztora, A. (1996). Surface order large deviations for Ising, Potts and percolation models. Probability Theory and Related Fields 104, 427-466.

317. Pokorny, M., Newman, C. M., and Meiron, D. (1990). The trapping ransition in dynamic (invasion) and static percolation. Journal of Physics A: Mathematical and General 23, 1431-1438.
318. Potts, R. B. (1952). Some generalized order-disorder transformations. Proceedings of the Cambridge Philosophical Society 48, 106-109.

319. Preston, C. J. (1974). Gibbs States on Countable Sets. Cambridge University Press, Cambridge

320. Propp J. G. and Wilson, D. B. (1995). Exact sampling with coupled Markov chains and applications to statistical mechanics. Random Structures and Algorithms (to appear)

321. Quas, A. (1996). Some properties of Lorentz lattice gas models (to appear). 322. Reimer, D. (1995). Butterflies (to appear).

323. Roy, R. and Meester, R. (1994). Uniqueness of unbounded occupied and vacant co

324. Roy, R. and Meester, R. (1996). Continuum Percolation. Cambridge University Press, Cambridge.

325. Ruijgrok, T. W. and Cohen, E. G. D. (1988). Deterministic lattice ga models. Physics Letters A 133, 415-418.

326. Russo, L. (1978). A note on percolation. Zeitschrift für Wahrscheinlichkeitstheorie und Verwandte Gebiete 43, 39-48.

327. Russo, L. (1981). On the critical percolation probabilities. Zeitschrift für Wahrscheinlichkeitstheorie und Verwandte Gebiete 56, 229-238.

328. Russo, L. (1982). An approximate zero-one law. Zeitschrift für Wahrscheinlichkeitstheorie und Verwandte Gebiete 61, 129-139.

329. Scheinerman, E. R. and Wierman, J. C. (1987). Infinite AB percolation clusters exist. Journal of Physics A: Mathematical and General 20 1305-1307.

330. Schonmann, R. H. (1994). Theorems and conjectures on the droplet-driven relaxation of stochastic Ising models. Probability and Phase Transition (G. R. Grimmett, ed.), Kluwer, Dordrecht, pp. 265-310.

331. Seymour, P. D. and Welsh, D. J. A. (1978). Percolation probabilities on the square lattice. Advances in Graph Theory (B. Bollobás, ed.), Annals of Discrete Mathematics 3, North-Holland, Amsterdam, pp. 227-245.

332. Simon, B. (1980). Correlation inequalities and the decay of correlations in ferromagnets. Communications in Mathematical Physics 77, 111-126.

333. Sinai, Ya. G. (1982). Theory of Phase Transitions: Rigorous Results. Pergamon Press, Oxford.

334. Slade, G. (1991). The lace expansion and the upper critical dimension for percolation. Mathematics of Random Media (W. E. Kohler and B. S. White, eds.), Lectures in Applied Mathematics, vol. 27, American Mathematical Society, Providence, pp. 53-63.

335. Slade, G. (1995). Bounds on the self-avoiding walk connective constant, Special Issue: Proceedings of the Conference in Honor of Jean-Pierre Kahane, 1993. Journal of Fourier Analysis and its Applications, 525-533.

336. Stacey, A. M. (1996). The existence of an intermediate phase for the contact process on trees. Annals of Probability 24, 1711-1726.

337. Stauffer, D. (1981). Scaling properties of percolation clusters. Disordered Systems and Localization (C. Castellani, C. DiCastro, and L. Peliti, eds.), Springer, Berlin, pp. 9-25. 
338. Swendsen, R. H. and Wang, J. S. (1987). Nonuniversal critical dynamics in Monte Carlo simulations. Physical Review Letters 58, 86-88.

339. Sykes, M. F. and Essam, J. W. (1964). Exact critical percolation probabilities for site and bond problems in two dimensions. Journal of Mathematical Physics 5, 1117-1127.

340. Tanemura, H. (1995). Critical behavior for a continuum percolation model (to appear).

341. Vahidi-Asl, M. Q. and Wierman, J. C. (1990). First-passage percolation on the Voronoi tesselation and Delaunay triangulation. Random Graphs '87, Wiley, London, pp. 341-359.

342. Vahidi-Asl, M. Q. and Wierman, J. C. (1990). A shape result for firstpassage percolation on the Voronoi tesselation and Delaunay triangulation. Random Graphs '89, Wiley, London, pp. 247-262.

343. Vahidi-Asl, M. Q. and Wierman, J. C. (1993). Upper and lower bounds for the route length of first-passage percolation in Voronoi tesselations. Bulletin of the Iranian Mathematical Society 19, 15-28.

344. Wang, F. and Cohen, E. G. D. (1995). Diffusion in Lorentz lattice gas cellular automata: the honeycomb and quasi-lattices compared with the square and triangular lattices. Journal of Statistical Physics 81, 467-495.

345. Watts, G. M. T. (1996). A crossing probability for critical percolation in two dimensions. Journal of Physics A: Mathematical and General 29, L363-I368.

346. Welsh, D. J. A. (1993). Percolation in the random-cluster process. Journal of Physics A: Mathematical and General 26, 2471-2483.

347. Whittle, P. (1986). Systems in Stochastic Equilibrium. John Wiley, Chichester

348. Whittle, P. (1994). Polymer models and generalised Potts-Kasteleyn models. Journal of Statistical Physics 75, 1063-1092

349. Wierman, J. C. (1987). Directed site percolation and dual filling models Annals of Discrete Mathematics $\mathbf{3 3}, 339-352$

350. Wierman, J. C. (1987). Duality for $k$-degree percolation on the square lattice. Percolation Theory and Ergodic Theory of Infinite Particle Systems (H. Kesten, ed.), Springer, Berlin, pp. 311-323.

351. Wierman, J. C. (1988). Bond percolation critical probability bounds derived by edge contraction. Journal of Physics A: Mathematical and General 21, 1487-1492.

352. Wierman, J. C. (1988). AB percolation on close-packed graphs. Journal of Physics A: Mathematical and General 21, 1939-1944.

353. Wierman, J. C. (1988). On AB percolation in bipartite graphs. Journal of Physics A: Mathematical and General 21, 1945-1949.

354. Wierman, J. C. (1989). AB percolation: a brief survey. Combinatorics and Graph Theory, vol. 25, Banach Centre Publications, Warsaw, pp. 241-251.

355. Wierman, J. C. (1990). Bond percolation critical probability bounds for the Kagomé lattice by a substitution method. Disorder in Physical Systems G. R. Grimmett and D. J. A. Welsh, eds.), Clarendon Press, Oxford, pp. $349-360$
356. Wierman, J. C. (1992). Equality of the bond percolation critical exponents for two pairs of dual lattices. Combinatorics, Probability, Computing 1, $95-105$.

357. Wierman, J. C. (1994). Equality of directional critical exponents in multiparameter percolation models. Journal of Physics A: Mathematical and General 27, 1851-1858.

358. Wierman, J. C. (1995). Substitution method critical probability bounds for the square lattice site percolation model. Combinatorics, Probability, Computing 4, 181-188.

359. Wilson, R. J. (1979). Introduction to Graph Theory. Longman, London.

360. Wu, F. Y. (1982). The Potts model. Reviews in Modern Physics 54, 235-268.

361. Yang. W and Zhang, Y (1992). A note on differentiability of the cluster density for independent percolation in high dimensions. Journal of Statistical Physics 66, 1123-1138.

362. Zhang, Y. (1991). A power law for connectedness of some random graphs at he critical point. Random Structures and Algorithms 2, 101-119.

363. Zhang, Y. (1992). Failure of the power laws on some subgraphs of the $Z^{2}$ lattice. Journal of Physics A: Mathematical and General 25, 6617-6622.

364. Zhang, Y. (1993). A shape theorem for epidemics and forest fires with finite range interactions. Annals of Probability 21, 1755-1781.

365. Zhang, Y. (1994). A note on inhomogeneous percolation. Annals of Probability 22, 803-820.

366. Zhang, Y. (1994). Analyticity properties at the supercritical state, preprint.

367. Zhang, Y. (1995). The fractal volume of the two-dimensional invasion percolation cluster. Communications in Mathematical Physics 167, 237-254.

368. Zhang, Y. (1995). Supercritical behaviors in first-passage percolation. Stochastic Processes and their Applications 59, 251-266.

369. Zhang, Y. (1995). A limit theorem for matching random sequences allowing deletions. Annals of Applied Probability 5, 1236-1240.

370. Zhang, Y. (1996). The complete convergence theorem on trees. Annals of Probability 24, 1408-1443.

371. Zhang, Y. (1996). Continuity of percolation probability in $\infty+1$ dimensions Journal of Applied Probability 33, 427-433.

372. Zhang, Y. (1996). Two critical behaviors of first passage time, preprint

373. Zhang, Y. (1996). Some power laws on two dimensional critical bond percolation, preprint.

374. Zhang, Y. (1996). Divergence of the bulk resistance at criticality in disordered media. Journal of Statistical Physics 84, 263-267.

375. Zhang, Y. and Zhang, Y. C. (1984). A limit theorem for $N_{0 n} / n$ in first passage percolation. Annals of Probability 12, 1068-1076.

376. Ziff, R. M., Kong, X. P., and Cohen, E. G. D. (1991). Lorentz lattice-gas and kinetic-walk model. Physical Review A 44, 2410-2428. 